\title{
Polaronic behaviour at manganite interfaces studied by advanced Raman scattering techniques
}

\author{
Dissertation \\ zur Erlangung des mathematisch-naturwissenschaftlichen Doktorgrades \\ "Doctor rerum naturalium" \\ der Georg-August-Universität Göttingen \\ im Promotionsprogramm ProPhys \\ der Georg-August-University School of Science (GAUSS)
}

vorgelegt von

Sebastian Merten

aus Osterholz-Scharmbeck

Göttingen, 2019 


\section{Betreuungsausschuss}

Prof. Dr. Vasily Moshnyaga

I. Physikalisches Institut, Georg-August-Universität Göttingen

Prof. Dr. Claus Ropers

IV. Physikalisches Institut, Georg-August-Universität Göttingen

\section{Mitglieder der Prüfungskommission}

\section{Referent:}

Prof. Dr. Vasily Moshnyaga

I. Physikalisches Institut, Georg-August-Universität Göttingen

\section{Korreferent:}

Prof. Dr. Claus Ropers

IV. Physikalisches Institut, Georg-August-Universität Göttingen

\section{Korreferentin:}

Prof. Dr. Kathrin Dörr

Naturwissenschaftliche Fakultät II, Martin-Luther-Universität Halle-Wittenberg

\section{Weitere Mitglieder der Prüfungskommission}

Prof. Dr. Christian Jooß

Institut für Materialphysik, Georg-August-Universität Göttingen

Prof. Dr. Michael Seibt

IV. Physikalisches Institut, Georg-August-Universität Göttingen

Prof. Dr. Hans-Christian Hofsäss

II. Physikalisches Institut, Georg-August-Universität Göttingen

PD Dr. Martin Wenderoth

IV. Physikalisches Institut, Georg-August-Universität Göttingen

Tag der mündlichen Prüfung: 29.10.2019 


\section{Contents}

$\begin{array}{ll}\text { 1. Introduction } & 1\end{array}$

$\begin{array}{ll}\text { 2. Scientific Background } & 7\end{array}$

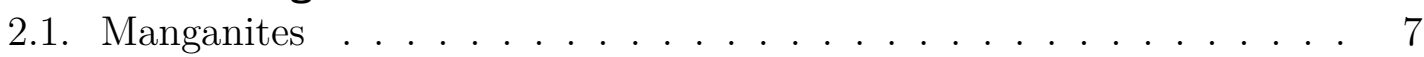

2.2. Raman scattering . . . . . . . . . . . . . . . . . . 23

2.3. Surface-enhanced Raman scattering . . . . . . . . . . . . . . . . . 31

2.4. Polaronic excitations probed by Raman spectroscopy: the status quo 40

3. Intrinsic antiferromagnetic coupling underlies colossal magnetoresistance effect - Role of correlated polarons 43

4. Magnetic-Field-Induced Suppression of Jahn-Teller Phonon Bands in $\left(\mathrm{La}_{0.6} \mathrm{Pr}_{0.4}\right)_{0.7} \mathrm{Ca}_{0.3} \mathrm{MnO}_{3}:$ the Mechanism of Colossal Magnetoresistance shown by Raman spectroscopy $\quad 59$

5. Metalorganic-aerosol-deposited Au nanoparticles for the characterization of ultrathin films by Surface-Enhanced Raman Spectroscopy $\quad 71$

6. Jahn-Teller reconstructed surface of the doped manganites shown by means of surface-enhanced Raman spectroscopy 83

$\begin{array}{ll}\text { 7. Conclusion } & 95\end{array}$

\begin{tabular}{ll}
\hline Bibliography & 111
\end{tabular}

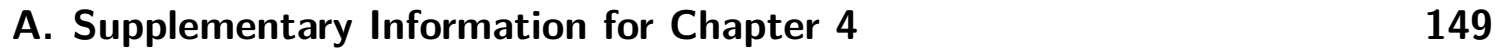

\begin{tabular}{ll}
\hline B. Supplementary Information for Chapter 6 & 157
\end{tabular}

C. Epitaxial single crystalline growth of $\mathrm{La}_{0.7} \mathrm{Sr}_{0.3} \mathrm{MnO}_{3}$ on $\mathrm{Al}_{2} \mathrm{O}_{3}(0001) \quad 165$

\begin{tabular}{ll}
\hline D. Author Contributions & 171
\end{tabular}

$\begin{array}{ll}\text { E. List of Publications } & 175\end{array}$ 



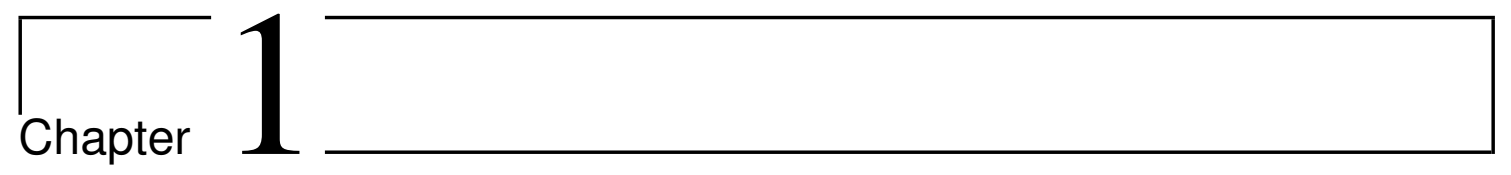

\section{Introduction}

Correlated behavior in transition metal oxides gives rise to a variety of fundamentally interesting phenomena, including charge- and spin-ordered states [1], hightemperature superconductivity [2, 3] and colossal magnetoresistance (CMR) [4], providing an exciting playing field for both, theoretical and experimental physicists. This can be illustrated in a simple schematic, shown in Fig.1.1 depicting the correlations between the different degrees of freedom, i.e. the spin, the electron, the orbital and the lattice. In principle, these correlations facilitate the manipulation of one or two of the degrees of freedom, e.g. spin and electron, by changing a third, e.g. the lattice. Therefore, by selectively opening or closing dissipation channels, e.g. phononic and electronic channels, fundamental principles of energy dissipation and their contributions to friction [5, 6], heterogeneous catalysis [7, 8], heat transport or photo-induced phenomena [9, 10] can be studied. Moreover, the emerging phenomena in correlated materials challenge consistently the current theoretical understanding of solid-state materials, leading to new advances in computational condensed matter physics [11,14].

In the majority of cases, one of the aforementioned correlations between the degrees of freedom is predominant and governs a certain phenomenon in correlated materials. While in multiferroics, materials exhibiting ferromagnetism and ferroelectricity simultaneously [16], the coupling between the spin system and the phononic degrees of freedom is dominant, superconductivity is controlled by the electronphonon coupling [2], i.e. the coupling between the lattice and the electron system. In many cases, the electron-phonon coupling is mediated by the formation of JahnTeller polarons. Originally discovered by TELLER and JAHN in 1937 [17], the JahnTeller (JT) effect lifts the orbital degeneracy of a transition metal ion acting in a crystal field by inducing a distortion of the lattice. This distortion, however, traps an electron in a self-inflicted potential minimum creating a bound state between the electron and the lattice, which is dubbed as a Jahn-Teller polaron [18]. For example, the strong electron-phonon coupling mediated by $\mathrm{Cu}^{2+}$, one of the strongest Jahn-Teller active ions [2], initiated the search of superconductivity in cuprates and 


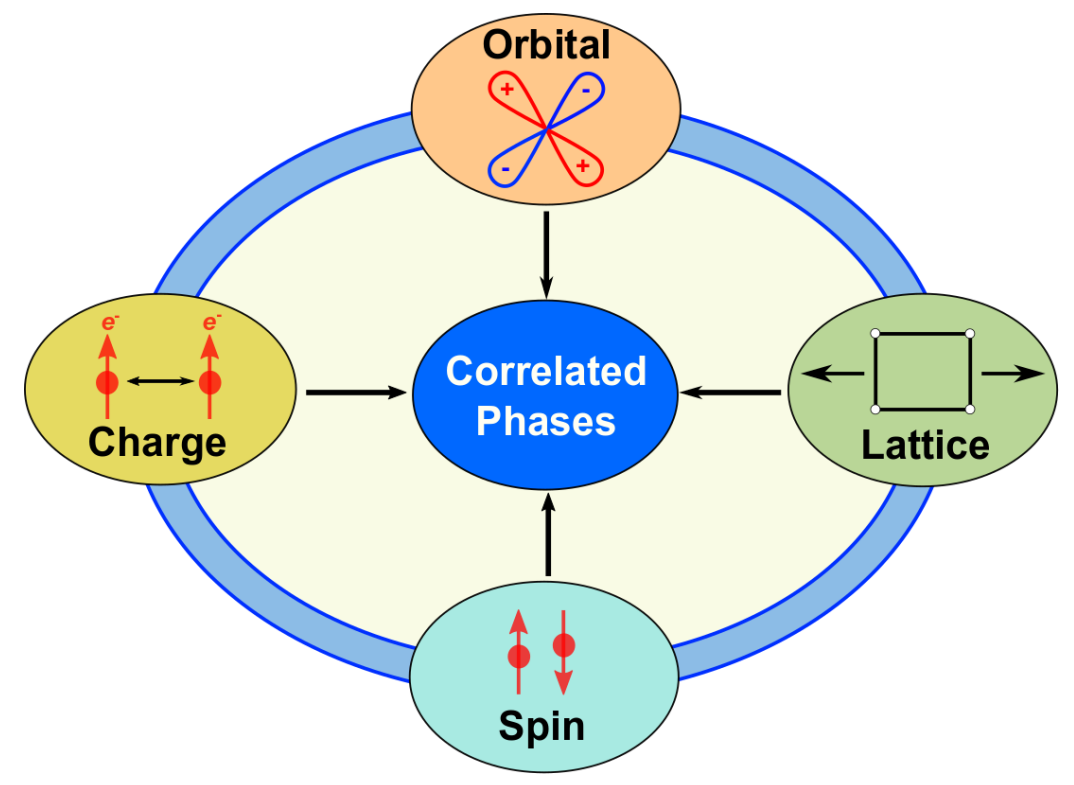

Figure 1.1.: The interplay of the electronic and structural degrees of freedom, namely the charge, spin, orbital and lattice degrees of freedom, gives rise to correlated phases in transition metal oxides. Reillustrated from [15].

resulted in the discovery of high-temperature superconductivity in $\mathrm{La}_{2-x} \mathrm{Ba}_{x} \mathrm{CuO}_{4}$ in 1986 by BEDNORZ and MüLleR [19]. Aside from high-temperature superconductivity, polaronic mechanisms play an important role in the mixed-valence manganites [20], as it is commonly believed that polaronic transport and the rapid creation and annihilation of polarons are mainly responsible for charge order (CO) phenomena, metal-insulator (MI) transitions and the CMR [20]. However, despite the enormous research effort since the rediscovery of the CMR effect [21 23], a complete model of manganite physics is still missing [24].

Beyond the fundamental interest in correlated oxides, the correlated behavior provides a new pathway for the realization of novel electronic devices, in which magnetism, superconductivity and the MI transition may be controlled by an applied electric or magnetic field [15. In principle, the coupling between the different degrees of freedom will not only allow researchers to study the fundamental processes in energy conversion, but may also serve as basis for novel logic devices in hard disk drives [15]. Considering two phases with dissimilar electronic characteristics, e.g. an insulating and metallic phase, the reversible switching between these two states induced by applying a magnetic field could be used for a rewritable memory cell. For this purpose in particular, the colossal magnetoresistance attracted much attention, since the resistivity of such CMR materials changes of several orders of magnitude in an applied magnetic field [22, 25, 26]. However, the practical application of manganites as magnetoresistive devices is inhibited by the low temperatures featuring the CMR $(T<273 \mathrm{~K})[1,21-23,26]$ as well as interface phenomena suppressing 
the electrical and magnetic properties [27 32 , the so-called dead layers. Although the probable origin of the dead layers lies within the symmetry-break at the surface and film/substrate interface and the occurring polar discontinuity or catastrophe [33, the mechanism which are compensating this discontinuity is still under debate [34, 35]. A detailed discussion of these mechanisms follows in Ch.2.1.3. Experimental results revealed, mainly, a compensation by segregation [36 38] and/or charge accumulation at the interface [39-44] as well as charge delocalization between substrate and manganite film [45, 46]. In mixed-valence manganites such an electron accumulation, however, corresponds to the enrichment of $\mathrm{Mn}^{3+}$ at the respective interface [4143], i.e. a Jahn-Teller active ion and a Jahn-Teller polaron, respectively. These results indicate that polarons, i.e. the strong electron-phonon coupling, not only play an important role in bulk phenomena, such as CMR, but also represent an important contribution to interfacial phenomena such as dead layers.

The nature of the correlated polarons, meaning an electron is strongly bound to a local lattice distortion, allows them to be studied by a variety of vibrational spectroscopy techniques, including Raman spectroscopy. In the past decades, vibrational spectroscopy has often been used to study the structure, dynamics and reactivity of crystalline materials, liquids, molecules and even amorphous samples [47 5 51]. The first spectra of molecular vibrations of organic liquids in the infrared region were acquired by ABNeY and Festing in 1881 [52]. Forty years later, C.V. RAman and K.S. KRISHNAN detected the inelastic scattering of light by liquids [53] and G. LANDSBERG and L. MANDELSTAM independently discovered the same phenomena in crystals [54] - however, only C.V. RAMAN was honored with the Nobel prize for this groundbreaking discovery in 1930. Today, Raman spectroscopy is one of the major characterization techniques in physics, chemistry and biology [55 57]. The diversity in modern-day applications is a particular consequence of modern technical advances of Raman instrumentation. The invention of monochromatic laser light sources, photomultipliers and charge-coupled devices as well as the development of triple monochromators improved substantially the quality and convenience of the recorded spectra. In the last years, Raman spectroscopy hence became a prominent and versatile tool in the field of condensed matter and material physics. Light scatters inelastically due to many fundamental excitations, such as collective lattice vibrations (phonons), spin waves and magnons, which is why their properties can be studied through a Raman spectrum [57].

In this thesis, Raman spectroscopy was applied to study the polaronic behavior in rare-earth manganites and, therefore, an overview of Raman spectroscopy is provided in Ch 2.2. The aim of this thesis is to give further insight into the long-standing issue of the electron-phonon coupling in mixed-valence manganites, its implication for the CMR and its relation to the microscopic and macroscopic interfaces in mixed-valence manganites. Polaronic excitations are represented by distinct phonon modes within the Raman spectrum [58, 59], whose temperatureand magnetic-field-dependent behavior provides information about the polaronic 
contributions to phenomena like CMR and MI transition. An overview of previous Raman studies of the polaronic behavior under several external perturbations is given in $\mathrm{Ch} 2.4$.

This cumulative thesis starts with the presentation of a mixed study, combining several experimental results such as structural characterization by x-ray diffraction, transmission electron microscopy, temperature-dependent Raman scattering experiments and detailed magnetotransport measurements. The corresponding results are presented in $\mathrm{Ch} 3$ and are constituted in the first publication of this cumulative thesis. Herein, it was shown that a tiny amount of correlated polarons $\left(n_{\mathrm{CP}}=0.5 \%\right.$ of the charge carriers) is sufficient enough to mediate an antiferromagnetic coupling, which is broken apart by aligning the ferromagnetic domains via an applied magnetic field or by decreasing the temperature. Temperature-dependent Raman scattering experiments in combination with the nonlinear electrical resistivity measurements ( $3 \omega$ signal) could show an increase of correlated polarons in the vicinity of the Curie temperature $T_{\mathrm{C}}$ and, thus, their correlation to the MI transition. However, at the beginning of this thesis, magnetic-field-dependent Raman studies on mixed-valence manganites exhibiting CMR were still missing. The only known magnetic-fielddependent study was focused on the melting of the long-range charge-/orbitally ordered $(\mathrm{COO})$ insulating phase in $\left(\mathrm{La}_{1-y} \mathrm{Pr}_{y}\right)_{1-x} \mathrm{Ca}_{x} \mathrm{MnO}_{3}(y=0.6, x=0.375)$ [60], which is accompanied by a structural transition from a monoclinic $\left(\mathrm{P} 2{ }_{1} / \mathrm{m}\right)$ to the orthorhombic (Pnma) structure. To complete the already existing temperaturedependent 61 66] and pressure-dependent [67] Raman studies of CMR manganites in the literature, a magnetic-field-dependent study of the prototypical CMR manganite $\left(\mathrm{La}_{0.6} \mathrm{Pr}_{0.4}\right)_{0.7} \mathrm{Ca}_{0.3} \mathrm{MnO}_{3}$ was therefore performed. The publication based on the results of this study is presented in Ch.4 and shows, unambiguously, the strong contribution of correlated polarons to the CMR effect. When applying a magnetic field in the vicinity of $T_{\mathrm{C}}$, a drastic decrease of the Raman-active JT stretching vibrations by $6000 \%$ was observed, which was in the order of the CMR (CMR $\approx 7000 \%$ ).

In addition, this thesis further provides the study of the polaronic behavior at the surface by more advanced Raman techniques. For this, a more advanced surfacesensitive Raman technique, called surface-enhanced Raman spectroscopy (SERS) 68 70, was used, which exploits the strong local enhancement of the electromagnetic field by plasmonic excitations in metallic nanostructures. The Raman scattering cross section is increased by the local coupling of the electric field of the excited surface plasmon with the incident and scattered light respectively, which results in an intensity enhancement by a factor of $|E|^{4}[68$, 71]. The strong distance dependence of the surface plasmon, i.e. its electric field $E(r)_{S P}$ scales with $1 / r^{3}$, limits the penetration depth to a few nanometers $(d<4-5 \mathrm{~nm})$ [72 74]. In the vicinity of two metallic nanostructures, so-called hot-spots, the enhancement may reach values in the order of $10^{8}-10^{12}$. [75] 78], allowing for the detection of single-molecules. A detailed discussion of the mechanisms underlying the intensity enhancement is given in Ch.2.3. Today, most SERS studies are performed in the field of biophysics 
[48, 79], medical and pharmaceutical research [80, where tiny amounts of drugs, bacteria or even DNA are characterized [76, 81]. However, studies on thin oxide films have barely been conducted [82, 83], since a noninvasive deposition of the required highly-active metallic nanostructures is a non-trivial task, making SERS studies quite challenging. Furthermore, to the best of the author's knowledge, only one tipenhanced Raman scattering study on the correlated double-perovskite $\mathrm{La}_{2} \mathrm{CoMnO}_{6}$ [84] has been published so far. This technique describes a similar technique as SERS, but combines it with the spatial resolution of an atomic force (AFM) or scanning tunneling microscope (STM). In this way, the plasmonic excitation is confined to the AFM/STM tip resulting in a higher spatial resolution compared to standard SERS experiments [69, 70]. A major obstacle in the application of SERS to correlated oxides is the creation of additional oxygen vacancies at the surface. Oxygen deficiencies have a huge impact on the electrical and magnetic properties of correlated oxides [85 88. Common deposition techniques such as pulsed-laser deposition (PLD) and sputtering require elevated temperatures and ultrahigh vacuum conditions (UHV), which often results in chemical segregation and/or the creation of oxygen vacancies in the oxide film [36-38, 87, 89 93]. These effects may be enhanced by depositing a metallic capping layer onto the oxide's surface [94 97]. Therefore, the presence of a metallic nanostructure may alter the surface properties of the studied correlated oxide, making the experimental result of the SERS study unreliable. Consequently, as a first step, a technique had to be developed to ensure noninvasive growth of the metallic nanostructures, preferably under ambient conditions, and thus preventing or at least minimizing the creation of oxygen vacancies and chemical segregation at the manganite surface.

In this thesis, the vacuum-free metalorganic aerosol deposition technique (MAD) [98] was chosen to enable the noninvasive growth of metallic nanoparticles on top of the manganite surface. Over the last years, the MAD has proven to be an ideal tool to grow various oxides, ranging from single oxide films [99] to complex heterostructures [100, 101]. The continuous oxygen-flow provided by the MAD should minimize deoxygenation effects and thus may be the perfect technique to allow SERS studies on correlated oxides. The current state of the MAD was extended to the deposition of metallic nanoparticles by using gold(III)acetate precursors. Chapter 5 demonstrates the growth of gold nanoparticles (Au-NPs) and its application to thin film characterization at the example of the well-known model system $\mathrm{TiO}_{2}$. The results presented in Ch.5 are published in manuscript No.3 of this cumulative thesis. The MAD-based approach produces highly active SERS substrates with an average enhancement factor of $10^{7}$, which enabled the characterization of a $3 \mathrm{~nm}$ thin $\mathrm{TiO}_{2}$ film.

The fourth and last manuscript presented in this thesis focuses on the surface properties of the mixed-valence manganites $\mathrm{La}_{0.7} \mathrm{Ca}_{0.3} \mathrm{MnO}_{3}$ and $\mathrm{La}_{0.7} \mathrm{Sr}_{0.3} \mathrm{MnO}_{3}$ probed by SERS and constitutes the highlight of this thesis. The aforementioned MAD-based growth of gold nanoparticles was applied allowing the first SERS study on a correlated oxide material presented in Ch.6. A Jahn-Teller distorted surface 
structure could be demonstrated in both manganite systems with a thickness of 47 u.c., which agrees well with the thicknesses of a suppressed magnetization at the surface observed in x-ray magnetic circular dichroism [93, 102] and x-ray resonant magnetic scattering [103, 104] experiments. Therefore, polaron formation has to be taken into account to describe the mechanisms underlying the dead layer formation and indicates a preferred accumulation of polarons at the interface.

Before starting the discussion of the scientific background of this thesis, a general remark to the structure of this cumulative thesis shall be given. The scientific publications on which this thesis is build, are presented in Ch.3.6. To lighten the access to the experimental results of the publications, a short motivation and summary of the relevant scientific results is given at the beginning of each chapter. Concluding this thesis, a general discussion of the published results is given (see Ch.7.1), which embeds them in the current knowledge of manganite physics. Furthermore, a short outlook of future prospects and a general summary of this work finalizes this thesis (see Ch.7.2). 


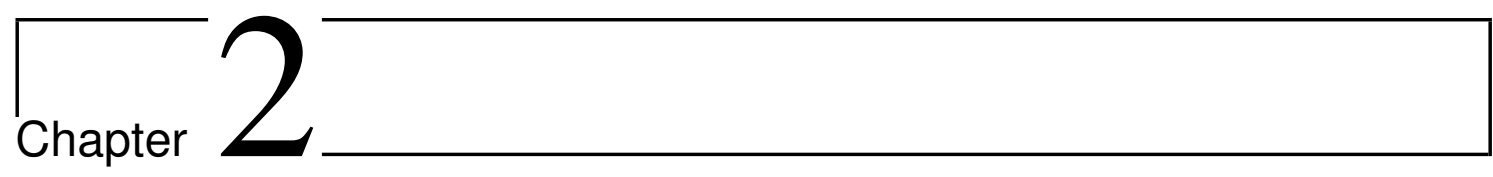

\section{Scientific Background}

In the following chapter, the scientific background for the experiments and results discussed in this thesis shall be presented. Starting from a brief description of the general structure and exchange mechanisms in manganites, the picture of electronically phase separated domains coupled by correlated polarons will be introduced. Subsequently, a brief summary of the relevant interface phenomena will be given. This is followed by the theoretical basics of conventional and surface-enhanced Raman spectroscopy, respectively. Finally, the possibilities of Raman spectroscopy in polaronic research are described by acknowledging the current experimental results, which are then embedded into the context of electron-phonon coupling and electronic phase separation in mixed-valence manganites.

\subsection{Manganites}

In general, the rare-earth manganites with the stoichiometric formula $\mathrm{AMnO}_{3}$ crystallize in the perovskite structure, shown in Fig.2.17 [26]. The manganese ion occupies the corner of the unit cell and is surrounded by six oxygen ions forming an octahedron, whereas the A-site cation is body-centered. The structural properties of manganites depend on the ionic radius $r_{A}$ of the A-site cation and its corresponding bond length to the oxygen ion $d_{\mathrm{A}-\mathrm{O}}$ as well as on its ratio to the manganese-oxygen bond length $d_{\mathrm{Mn}-\mathrm{O}}$. A parameter to classify structural distortions of the perovskite structure is the so-called tolerance factor $f$ [105], which is defined as $f=d_{\mathrm{A}-\mathrm{O}} /\left(\sqrt{2} d_{\mathrm{Mn}-\mathrm{O}}\right)$. The ideal, undistorted cubic perovskite structure manifests itself for $f=1$. An increasing distortion is accompanied by tilts of the oxygen octahedron, which can result in a rhombohedral $(0.96<f<1)$ or an orthorhombic structure $(f<0.96)[26]$. Such a tilting has not only a strong impact on the structural properties, but on the electrical and magnetic properties as well [106]. A tilt of the octahedron reduces the ideal Mn-O-Mn bond angle of $180^{\circ}$ and, thus, the overlap of the manganese and oxygen orbitals. 

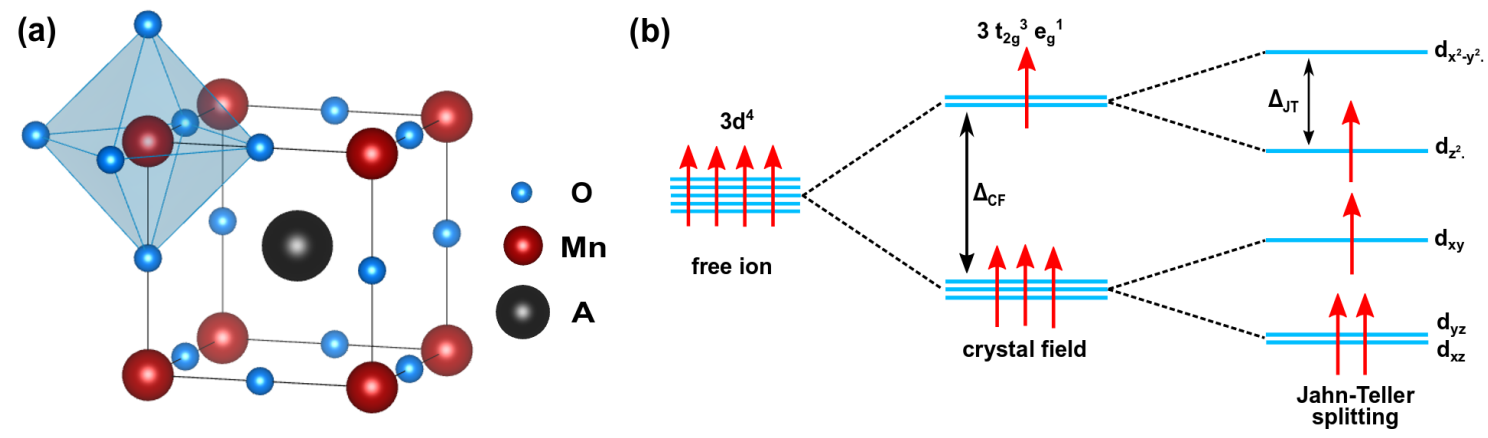

Figure 2.1.: (a) Ideal, cubic perovskite structure with chemical formula $\mathrm{AMnO}_{3}$, drawn with the help of VESTA [107]. (b) The five-fold degeneracy is lifted by the crystal-field which splits the $3 \mathrm{~d}$-orbitals into the triply degenerated $t_{2 \mathrm{~g}}$ and doubly degenerated $e_{\mathrm{g}}$-orbitals. The Jahn-Teller distortion further lifts each degeneracy.

In the stoichiometric $\mathrm{A}^{3+} \mathrm{Mn}^{3+}\left(\mathrm{O}^{2-}\right)_{3}$ composition the manganese ion possesses the electron high-spin configuration $[\mathrm{Ar}] 3 \mathrm{~d}^{4}\left(t_{2 \mathrm{~g}}^{3} e_{\mathrm{g}}^{1}\right)$ with one electron in the twofold degenerated $e_{\mathrm{g}}$ orbitals [26] (see Fig.2.1p). Naturally, the degeneracy is lifted by a Jahn-Teller distortion of the oxygen octahedra [17. If the number of JT ions is large enough, the distortions cannot be considered individually anymore. A cooperative Jahn-Teller effect emerges which is accompanied by a structural phase transition [108, 109]. In $\mathrm{LaMnO}_{3}$ for example, this phase transition occurs at $T \approx 800 \mathrm{~K}$ [108, 109]. A reduction of the JT ions, e.g. through doping with a divalent alkaline earth metal $(\mathrm{Ca}, \mathrm{Sr}, \mathrm{Ba}, \ldots)$, leads to a decrease of the transition temperature [109, 110]. Furthermore, the doping creates a mixed-valence state of the manganese ion with $\mathrm{Mn}^{4+}$ as a non-Jahn-Teller-distorted ion. However, for a small doping the octahedron of the $\mathrm{Mn}^{4+}$ ion adopts the cooperative distortion. Since the JT distortion traps the electron in a self-induced potential energy minimum, the electron is bound to the lattice, which is dubbed as a Jahn-Teller polaron. The formation of such a JT polaron and the mixed-valence state mainly governs the electrical and magnetic properties of the manganites [110].

\subsubsection{Exchange interactions}

The electrical and magnetic properties can be explained by the exchange interactions, which take a possible mixed-valence state of the manganese ion into account. The electron exchange is mediated by the p-orbitals of the oxygen ions and the $e_{\mathrm{g}}$-orbitals of the manganese making it an indirect interaction. The $t_{2 \mathrm{~g}}$ spins are strongly localized and hence do not take part in the exchange interaction. In a first instance, the indirect interaction between ions of same valence will be discussed, which was first introduced by Goodenough, Kanamori and Anderson. They further established a certain set of rules, the GKA rules, which allows the estimation of the strength and nature of the magnetic coupling [108, 111, 112]. 

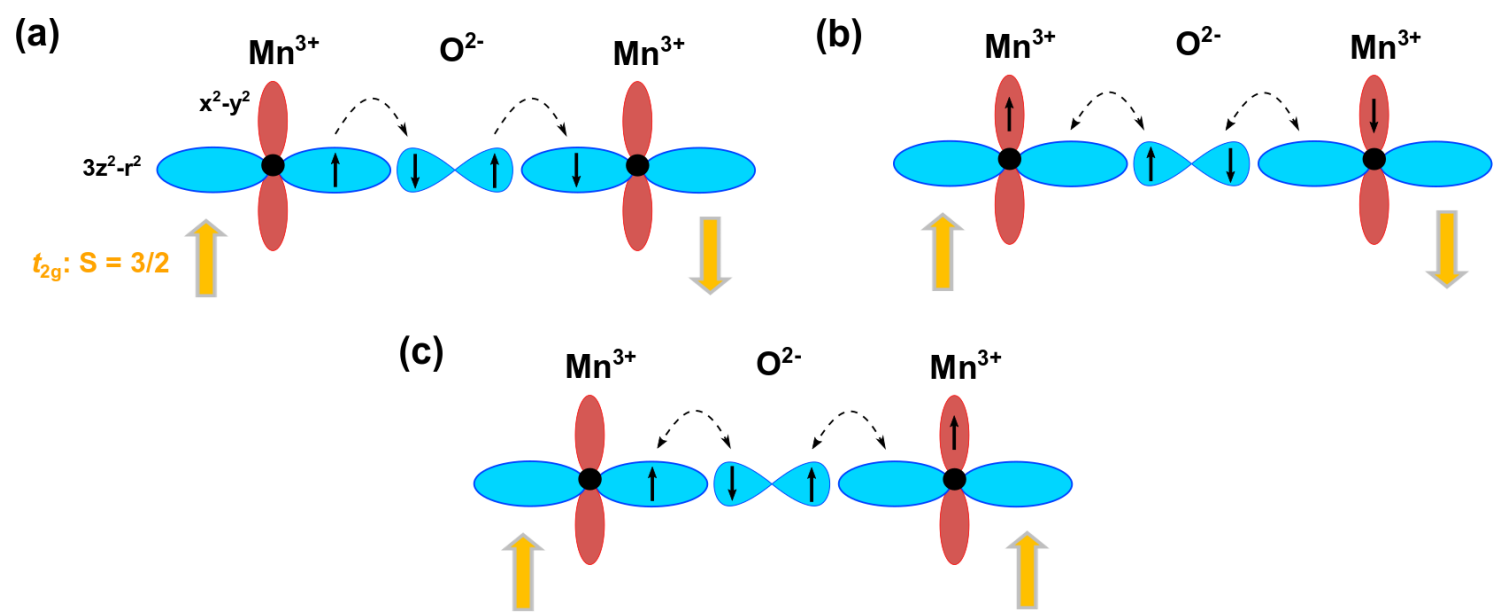

Figure 2.2.: Superexchange and the Goodenough-Kanamori-Anderson (GKA) rules explained by means of a $\mathrm{Mn}^{3+}$ bonding: the virtual hopping process (dashed arrows) between the $e_{\mathrm{g}}$ and $\mathrm{O}_{2 \mathrm{p}}$ levels mediate an exchange coupling. The $t_{2 \mathrm{~g}}$ rump spins follow the alignment of the $e_{\mathrm{g}}$ electrons due to Hund's coupling. (a) Hopping processes are only allowed, if the spins are aligned antiparallel due to Pauli's principle. (b) Hund's coupling governs an antiparallel spin alignment for occupied $3 \mathrm{z}^{2}-\mathrm{r}^{2}$ orbitals. (c) A ferromagnetic coupling is obtained when the $3 z^{2}-r^{2}$ and the orthogonal $\mathrm{x}^{2}-\mathrm{y}^{2}$ orbital are occupied. Adapted from [113, 114].

The superexchange interaction can be illustrated at the example of $\mathrm{LaMnO}_{3}$, where all $\mathrm{Mn}$ ions reside in the $\mathrm{Mn}^{3+}$ state. The hopping of an electron between the $e_{\mathrm{g}}$-orbitals of the $\mathrm{Mn}$ ions would lead to a twofold occupation of the $e_{\mathrm{g}}$-orbital and thus, to an increase of the Coulomb energy $U$. Since the Coulomb energy in $\mathrm{LaMnO}_{3}$ is much higher than the energy gain $t$ due to delocalization of the electrons $(U \approx 3.1 \mathrm{eV}, t \approx 0.4 \mathrm{eV}[115])$, its ground state can be described as a Mott-Hubbard insulator [116-118]. Therefore, each $e_{\mathrm{g}}$-orbital is occupied by just one electron. The spin of these electrons can now be either parallelly or antiparallelly aligned. In the parallel configuration, an exchange between the $e_{\mathrm{g}}$-orbitals is not allowed due to Hund's coupling. In contrast, a virtual hopping process can occur for an antiparallel alignment, where the electrons of the adjacent $e_{\mathrm{g}}$-orbitals are swapped. This results in an energy gain $\Delta E=-2 t^{2} / U[113,114$ and is shown in Fig.2.2 a. However, the $e_{\mathrm{g}}$ electrons can also be located in the orbital perpendicular to the $\mathrm{Mn}-\mathrm{O}-\mathrm{Mn}$ bond, so that two unoccupied orbitals face each other at the oxygen bond. This is depicted in Fig.2.2b. These two cases are summarized in the first GKA rule, which describes the $180^{\circ}$ exchange between two empty (or two occupied orbitals) leading to an antiferromagnetic coupling. Figure 2.2 c depicts the interaction between an empty and an occupied orbital. In this case a ferromagnetic coupling is preferred due to Hund's coupling. The third GKA rule applies to the $90^{\circ}$ exchange, which is not relevant for the manganites due to geometric reasons [113, 114] and is, therefore, 
left out in Fig,2.2. The $t_{2 \mathrm{~g}}$ rump spins follow the alignment of the $e_{\mathrm{g}}$ electrons due to Hund's coupling. With the help of the GKA rules, many three dimensional spin arrangements can be predicted, e.g. the A-type antiferromagnetic coupling in $\mathrm{LaMnO}_{3}$ [113, 114] or the ferromagnetic, insulating ground state of the doubleperovskite $\mathrm{La}_{2} \mathrm{Ni}(\mathrm{Co}) \mathrm{MnO}_{6}$ [119, 120].

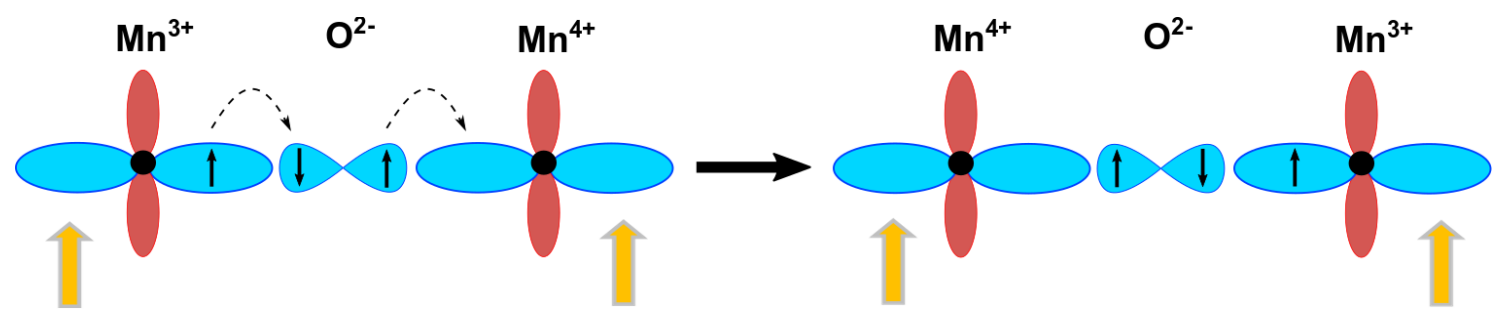

Figure 2.3.: For parallel alignment of the $t_{2 \mathrm{~g}}$ spins of adjacent Mn ions, a hopping of the $e_{\mathrm{g}}$ electrons from the $\mathrm{Mn}^{3+}$ to the $\mathrm{Mn}^{4+}$ is allowed, mediating electron transfer. Reillustrated from [113, 114]

The doping of the parent compound $\mathrm{LaMnO}_{3}$ with a divalent earth alkaline metal such as calcium or strontium creates a mixed-valence state and formally, one $e_{\mathrm{g}}$ electron is switched with a hole [110]. The mixed-valence state cannot be described by the superexchange mechanism anymore and has a major impact on the electrical and magnetic properties of the manganite. The first theoretical works concerning the mixed-valence state were made independently by ZENER [121], by ANDERSON and Hasegawa [122] as well as by De Gennes [123], all consistently predicting a ferromagnetic, metallic ground state. They named this novel exchange interaction between two ions of different valence, the double-exchange mechanism (DEX). A schematic representation of the possible spin arrangement is shown in Fig.2.3. Since the $e_{\mathrm{g}}$ orbital of the $\mathrm{Mn}^{4+}$ ion is unoccupied, electrons can hop between both $\mathrm{Mn}$ ions via the p-orbital of the oxygen leading to a net current. However, the transport would not occur, if the localized $t_{2 \mathrm{~g}}$ spins are antiferromagnetically aligned due to Hund's coupling energy. Hence, the double-exchange mechanism favors a ferromagnetic, metallic ground state. As a consequence of the strong Hund's coupling, the orientation between the spin at lattice site $i$ and the localized spins at site $j$ plays an important role. Here, the hopping element $t$, i.e. the kinetic energy, is substituted with an effective hopping element $t_{\text {eff }}$, which depends on the angle $\Theta_{i j}$ between two adjacent localized $t_{2 \mathrm{~g}}$ spins

$$
t_{\mathrm{eff}}=t \cdot \cos \left(\frac{\Theta_{i j}}{2}\right)
$$

As one can easily see, $t_{\text {eff }}$ is maximal for a parallel alignment, but vanishes for an antiparallel alignment. 


\subsubsection{Polaronic behavior and electronic phase separation}

Although, the double-exchange mechanism can qualitatively explain the occurrence of a ferromagnetic, metallic ground state, it neglects the electron-phonon coupling, which is present due to the mentioned formation of Jahn-Teller polarons. Furthermore, it falls short of describing phenomena such as the strong change of the electrical transport at the Curie temperature $T_{\mathrm{C}}$ or the resistivity behavior in an applied external magnetic field [124]. By introducing the electron-phonon coupling via a dimensionless parameter $\lambda=E_{\text {latt }} / t_{\text {eff }}$ [125, 126], with $E_{\text {latt }}$ as the energy gain due to the localization of an electron in a JT distorted octahedron, MILLIS et al. extended the double-exchange model to polaronic effects. With the help of this parameter $\lambda$, they were able to explain the insulating character at high temperatures [127] as well as the behavior of the resistivity and magnetic transition temperature of $\mathrm{La}_{1-x} \mathrm{Sr}_{x} \mathrm{MnO}_{3}$ [109]. Introducing the electron-phonon coupling into the physical description of manganites, the tolerance factor $f$ may be considered as a structural parameter, indicating the relative strength of the DEX and the electron-lattice interactions (EL) [128]. Based on this fact, the manganites can be classified into large bandwidth (DEX > EL), intermediate bandwidth $(\mathrm{DEX} \approx \mathrm{EL})$ and small bandwidth $(\mathrm{DEX} \ll \mathrm{EL})$ systems.

The formation of Jahn-Teller polarons was first observed by neutron powderdiffraction experiments in $\mathrm{La}_{1-x} \mathrm{Ca}_{x} \mathrm{MnO}_{3}$ with different dopings $(x \leq 0.25)$ [129, 130]. Furthermore, the thermally activated hopping of small polarons [131] above the metal-insulator transition temperature $T_{\mathrm{MI}}$ and their delocalization [132] as well as the remaining polaronic signatures below $T_{\mathrm{MI}}$ [133 135] suggests that the polarons can be considered as the "charge carriers" in mixed-valence manganites. The thermally activated small polaron hopping was further verified by optical conductivity [136 138], thermoelectric experiments and Hall coefficient measurements [139] 141]. However, in the weak coupling regime, e.g. as in $\mathrm{La}_{0.7} \mathrm{Sr}_{0.3} \mathrm{MnO}_{3}$, the small polaron signature in the mid-infrared is suppressed and excitations of rather delocalized polarons are present. These delocalization polarons, sometimes dubbed as large polarons, manifest itself as a Drude-like absorption feature [134, 135]. Isotope experiments, where ${ }^{16} \mathrm{O}$ was substituted by ${ }^{18} \mathrm{O}$, supported the consideration of polarons as the main charge carriers further, as the isotope substitution results in a reduction of $T_{\mathrm{MI}}$ by $21 \mathrm{~K}[142,143$. This substitution increases the local distortions, which leads to a higher splitting of the $e_{\mathrm{g}}$-orbitals and thus to a higher electron localization. Such a strong decrease is not observable when the JT effect is negligible small, such as in $\mathrm{SrRuO}_{3}$.

A bipolaronic nature of the polarons was proposed based on x-ray and neutron scattering studies [144-146], which indicated the formation of short-range correlations of a few nanometers. This bipolaronic state (alternatively called a correlated polaron) can be considered as a short-ranged charge/orbital ordered superstructure, which is shown in Fig 2.4 a. It exhibits an orbital order similar to the charge-exchange 
(CE) phase of half-doped $\mathrm{La}_{0.5} \mathrm{Ca}_{0.5} \mathrm{MnO}_{3}$ [147, which may be of stripe-like geometry [148 and possesses antiferromagnetic correlations at a length scale of $d_{\mathrm{CP}} \approx 1-2 \mathrm{~nm}$ [146]. Taking these correlation effects into account, a direct relation between the MI transition and polaronic effects could be established, since the amount of correlated polarons rapidly increases with increasing temperatures, but saturates above $T_{\mathrm{MI}}$ [145]. Moreover, the correlations may cause the intrinsic non-linearity in the electrical transport, which is drastically enhanced in the vicinity of the MI transition [149], the softening of the bulk modulus near $T_{\mathrm{C}}$ [150] and are presumably responsible for the acoustic damping in $\mathrm{La}_{0.7} \mathrm{Ca}_{0.3} \mathrm{MnO}_{3}$ [151].
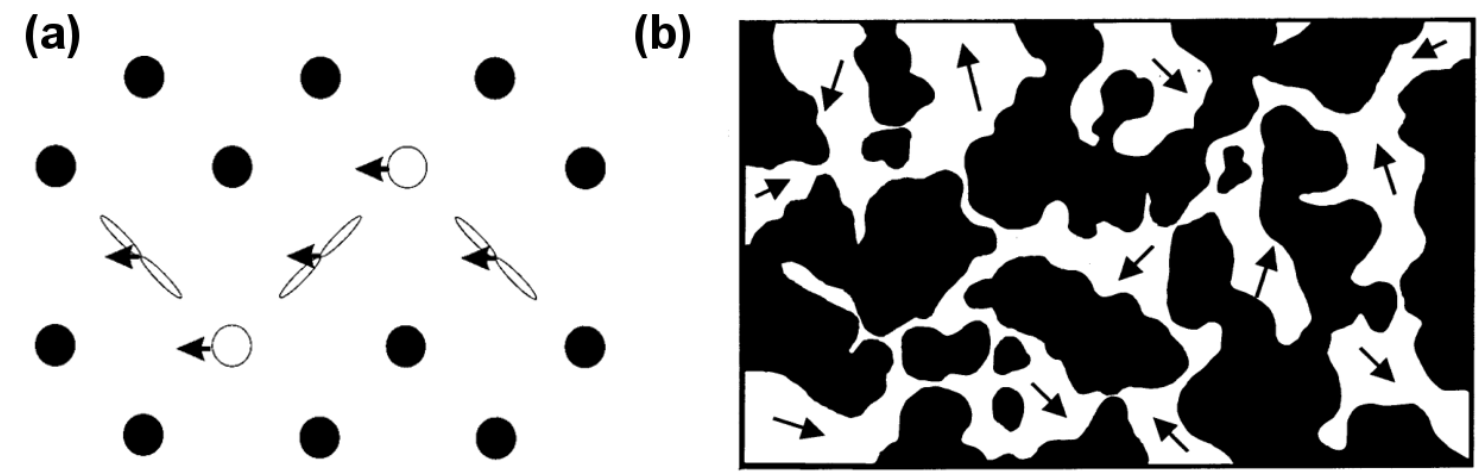

Figure 2.4.: (a) Schematic diagram of a bipolaronic structure of CE-type, i.e. the correlated polarons. Open circles represent $\mathrm{Mn}^{4+}$ and elongated figureeights the occupied $e_{\mathrm{g}}$ orbital of the $\mathrm{Mn}^{3+}$ ion. Arrows signify the magnetic moment and closed circles the Mn ions, which have the formal valence and no net orbital order. Adapted from [146] with kind permission of The American Physical Society. (b) Illustration of the electronic phase separation of CE-type (dark areas) and ferromagnetic metallic (white areas) domains. Taken from [152] with kind permission of Springer Nature.

Pulsed neutron diffraction [153] and x-ray absorption spectroscopy [154] indicated the existence of remnants of Jahn-Teller polarons in the metallic phase, which was supported by isotope effects observed in resistivity [155], thermoelectric power measurements [156] and the remaining polaronic character of the charge carriers below $T_{\mathrm{MI}}$ [133]. It was proposed that this pronounced coexistence in the paramagnetic regime may be due to a dynamic phase separation between antiferromagnetically coupled insulating regions, in which the bipolarons/correlated polarons reside and small ferromagnetic regions, where the non-correlated small polarons are located (see Fig 2.4b). The creation of disorder within the manganite system was conjectured to cause the formation of electronically dissimilar clusters [157. Such disorder can be induced by chemical doping, which, in turn, tunes the electron-phonon coupling within the manganite system. Thus, the electronic phase separation is assumed to be an intrinsic instability of mixed-valence manganites and correlated oxides in general [158]. SEN et al. could clearly identify both electronically separated phases as 
charge-ordered insulating and ferromagnetic metallic states, which compete strongly with each other [159]. At a certain critical point, the competition results in a first order phase transition [160]. However, inducing disorder into such a system opens a gap between the two ordered states and an intermediate state arises, where the CMR develops [160]. The influence of this disorder is schematically shown in Fig 2.5. In the absence of or a very weak disorder in the system (Fig. 2.5a), a transition between the two competing phases can be driven by changing the order parameter $g$, e.g. the electron-phonon coupling, the applied magnetic field and so on. With increasing disorder, the temperature of the first order phase transition is decreased and a second characteristic temperature $T^{*}$ is revealed, where CO clusters start to form (Fig.2.5p). In the limit of substantial disorder (Fig,2.5c), a gap between both states opens creating an intermediate state. Because of the huge disorder within this state, it can be referred to as a polaronic glass [1, 144, 160], which consists of coexisting clusters of both highly competing phases.
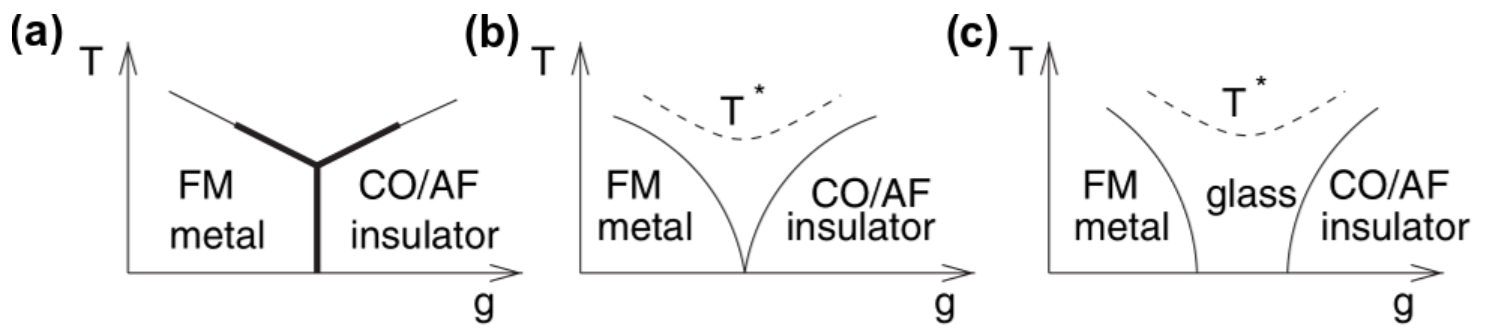

Figure 2.5.: A generic phase diagram of the two competing phases. (a) In the absence of disorder, a first order transition occurs between the two ordered phases. Here, $g$ represents an ordering parameter, which drives the transition between the two phases. (b) Increasing the disorder lowers the transition temperature of the first order phase transition and may even result in a quantum critical point. (c) Substantial disorder opens a gap between the two states, giving rise to a glassy intermediate state, where CMR develops. Adapted from [160].

The competition of both phases as well as the glassy nature of this intermediate state was further emphasized by tracking the metal-insulator transition with photoemission electron microscopy and resonant elastic soft x-ray scattering [161]. This competition is commonly believed to be the origin of CMR, as it could explain the extremely large CMR ratios [162, 163] of $10^{5}-10^{7} \%$ in systems with a strong electron-phonon coupling, such as $\left(\mathrm{La}_{0.6} \mathrm{Pr}_{0.4}\right)_{0.7} \mathrm{Ca}_{0.3} \mathrm{MnO}_{3}$ [164]. Moreover, the electronic phase separation results in a percolative transport through the ferromagnetic domains [152, 165], which is strongly influenced by substrate-induced strain [166]. These effects may partly explain the observed strain- and thicknessdependence of the transition temperature in thin films [28, 167, 168]. Recently, it was shown that even a tiny amount of correlated polarons is able to mediate an intrinsic antiferromagnetic coupling between the nanometer sized ferromagnetic 
domains, yielding an increase of the resistivity at the MI transition temperature [66]. This will be discussed further in the first publication presented in $\mathrm{Ch} 3$ of this cumulative thesis. Such an antiferromagnetic coupling between the ferromagnetic metallic domains via correlated polarons could also explain the reduction of the optical reflectance by $18 \%$ in $\left(\mathrm{La}_{0.6} \mathrm{Pr}_{0.4}\right)_{0.7} \mathrm{Ca}_{0.3} \mathrm{MnO}_{3}$ [169].

Although the framework of correlated polarons within a phase separated material is a commonly believed picture for the explanation of CMR and the MI transition, an alternative model for describing the CMR proposed by AlEXANDRov et al. should be mentioned. Based on the observation that the charge carriers exhibit oxygen $2 \mathrm{p}$ hole character [170], the exchange interaction of polaronic holes with $\mathrm{d}$ electrons may result in the formation of bipolaronic oxygen holes [171, 172]. Whilst the temperature decreases towards $T_{\mathrm{C}}$, the strength of the exchange coupling increases and the bipolarons break apart, resulting in a ferromagnetic metallic state with delocalized small polarons. The ferromagnetic state, therefore, represents a polaronic conductor [171, 173]. With the help of this current carrier density collapse (CCDC) model [171, 172, AlEXANDrov et al. were able to explain the CMR effect in $\mathrm{La}_{0.75} \mathrm{Sr}_{0.25} \mathrm{MnO}_{3}$, the isotope effect, the shift of spectral weight in the mid -infrared region below the MI transition [174, 175] and the observed electronic phase separation [173]. Furthermore, a first order phase transition was predicted, which was later confirmed by specific heat measurements [176. Based on the CCDC, GRAziosi et al. described the electronic transport properties of $\mathrm{La}_{0.7} \mathrm{Sr}_{0.3} \mathrm{MnO}_{3}$ and $\mathrm{La}_{0.65} \mathrm{Ca}_{0.35} \mathrm{MnO}_{3}$ as a bipolaronic insulator in the paramagnetic regime and as a polaronic metal in the ferromagnetic phase [177]. With the help of the proposed model, they succeeded in remodeling the temperature-dependent resistivity of $\mathrm{La}_{0.7} \mathrm{Sr}_{0.3} \mathrm{MnO}_{3}$ and $\mathrm{La}_{0.65} \mathrm{Ca}_{0.35} \mathrm{MnO}_{3}$ in the temperature range $T=5-400 \mathrm{~K}$. However, such a bipolaronic hole would show a shorter correlation length than the one obtained by scattering experiments [146] since it resides on the same ion, thus being inconsistent with the suggested CE-type structure [146].

While the importance of polaronic effects, the electronic phase separation and the competition between delocalization and localization due to electron-phonon coupling is generally accepted for magnetoresistive manganites, the significance of the strength of the electron-phonon coupling is still under debate [178, 179]. As a largebandwidth manganite, $\mathrm{La}_{0.7} \mathrm{Sr}_{0.3} \mathrm{MnO}_{3}$ [180] was commonly believed to show weak electron-phonon coupling and negligible polaronic correlations [20, 110, 135]. However, in recent neutron and x-ray diffraction experiments it was argued that this might not be the case [178]. The observation of CE-type polarons, i.e. correlated polarons, in $\mathrm{La}_{0.7} \mathrm{Sr}_{0.3} \mathrm{MnO}_{3}$ [178] in a temperature range of $200 \mathrm{~K} \leq T \leq 350 \mathrm{~K}=T_{\mathrm{C}}$ suggested strong electron-phonon coupling, which could be a generic feature of the ferromagnetic metallic phase. The strength of the magnetoresistance may be primarily determined by the polaron lifetime and mobility, respectively, rather than the strength of the electron-phonon coupling. 


\subsubsection{Interface effects}

The huge resistivity change in CMR manganites has great potential for applications, e.g. as magnetoresistive switching devices in electronics and spintronics. For such devices, adequate preparation and implementation of thin manganite films into the existing microchip structure is necessary. A major obstacle for this integration turned out to be the so-called dead layers [28, 31, i.e. an interface-bound zone in which the electrical and magnetic properties are suppressed. They provide an electrostatic barrier at the interface, impeding the application of correlated oxides in magnetoresistive devices [27, 29, 30, 32] and heterogeneous catalysis [181-183]. Furthermore, they may also contribute to the decreasing transition temperature with decreasing film thickness [28]. Therefore, a brief summary of the interface properties will be given in the following chapter. Since this thesis mainly covers the polaronic behavior at the surface, a particular focus will lie on surface mechanisms, which show a similar behavior as compared to the dead layers at the interface. Additional phenomena occurring at such interfaces in heterostructures, e.g. the formation of a $2 \mathrm{D}$ electron gas [184], high- $T_{\mathrm{C}}$ ferromagnetism [101, 185] and skyrmions [186], are beyond the scope of this thesis and will, therefore, be neglected in the following discussion.

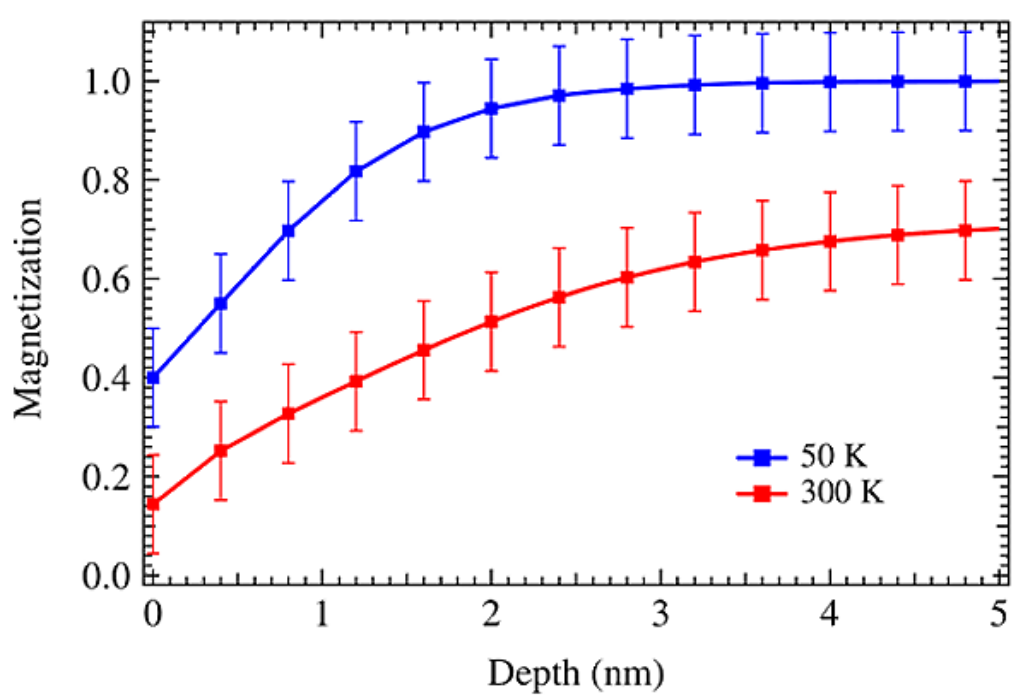

Figure 2.6.: XRMS measurements on $\mathrm{La}_{0.7} \mathrm{Sr}_{0.3} \mathrm{MnO}_{3} / \mathrm{SrTiO}_{3}(100)$ films reveal a degraded surface magnetization, which is reduced by decreasing the temperature. From [103] with the kind permission of IOP Publishing.

X-ray magnetic circular dichroism (XMCD) [102 and x-ray resonant magnetic scattering (XRMS) [103, 104] on thin $\mathrm{La}_{0.7} \mathrm{Sr}_{0.3} \mathrm{MnO}_{3} / \mathrm{SrTiO}_{3}(100)$ films verified a suppressed surface magnetization, i.e. a magnetic dead layer, at ambient temperatures, which is restored to its bulk value within $2-4 \mathrm{~nm}$ into the film (see Fig.2.6. Lowering the temperature decreases the thickness of the suppressed magnetization 
layer [103, 104, which can even lead to a bisection of its former size [103]. Additionally, conductive atomic force microscopy (c-AFM) [187] and contact resistance measurements [188] confirmed the existence of such an insulating layer at the manganite surface and indicated its intrinsic character. However, the results of the latter have to be interpreted carefully, since the contact resistance measurements required the deposition of a $30 \mathrm{~nm}$ thick $\mathrm{Pt}$ film on top of the manganite surface [188]. The deposition of the metallic capping layer itself [94 97] as well as the applied UHV deposition technique [188] may cause an oxygen deficient surface layer. Such oxygen vacancies, in turn, weaken the double-exchange mechanism and would, therefore, artificially increase the inferred thickness of the nonconducting layer, $d=3 \mathrm{~nm}$ [188. Typically, the magnetic dead layer is smaller than the electrical one [189], which is attributed to the electronic phase separation and percolative behavior at the corresponding interface [190-193]. While ferromagnetism is still observed for 4-5 monolayers [189], the experimentally determined lower bound for metallic behavior ranges between 7 and 8 monolayers [189, 194].

It is commonly believed that the origin of these dead layers lies in the symmetrybreaking at the oxide interface and the occurring polar catastrophe [33], i.e. the formation of an electrostatic potential. However, the mechanisms compensating the polar catastrophe and, thereby, creating the dead layers, are still a highly discussed topic in today's literature [35, 195]. The underlying fundamental problem occurring at an oxide interface is illustrated in Fig.2.7. Perovskite oxides along the [001] direction consist of alternating stacks of $\mathrm{AO}$ - and $\mathrm{BO}_{2}$-planes. In $\mathrm{SrTiO}_{3}$, for example, these layers are the neutrally charged $\left(\mathrm{Sr}^{2+} \mathrm{O}^{2-}\right)^{0}$ and $\left(\mathrm{Ti}^{4+} \mathrm{O}_{2}^{2-}\right)^{0}$. However, when its surface is cut along another direction, e.g. the (110) plane, the crystal consists of electrically charged planes, the $\left(\mathrm{O}_{2}^{2-}\right)^{4-}$ and $\left(\mathrm{Sr}^{2+} \mathrm{Ti}^{4+} \mathrm{O}^{2-}\right)^{4+}$ planes. As illustrated in Fig $2.7 \mathrm{a}$, the alternating order of charged planes results in an electrostatic potential $V$, which increases linearly with an increasing film thickness. Such a divergence of the electrostatic potential in ionic crystals is called polar discontinuity or polar catastrophe. Although the realization depends on the contributing materials and the growth conditions, the underlying principle is always similar and exemplarily shown in Fig 2.7b for a film consisting of charged planes on a neutrally charged substrate. The continuous increase of the potential $V$ is prevented by the transfer of the charge $-Q / 2$ from the surface to the interface. The resulting electric field $E$ oscillates around zero and the potential around a finite value, independent of the film's thickness.

The compensation of the build-up potential, however, depends on the nature of the polar surface itself and can be influenced by the growth conditions of the oxide film. While the (110)- $\mathrm{SrTiO}_{3}$ reconstructs by the formation of (001)-oriented nanofacets [196], the $\mathrm{NiO}(111)$ surface shows a octopolar reconstruction [197]. As a consequence, $1 / 4$ of the outer $\mathrm{Ni}^{2+}$ and $3 / 4$ of the $\mathrm{O}^{2-}$ ions are removed from the surface, leading to the formation of a charged surface plane (charge: +1$)$. In a similar way, the $\mathrm{LaMnO}_{3}(110)$ surface is stabilized by the formation of oxygen vacan- 
(a)

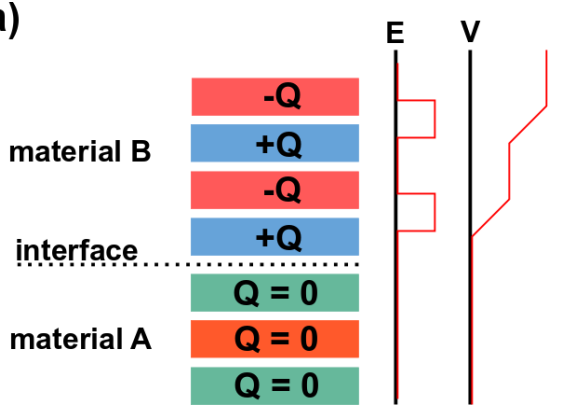

(b)

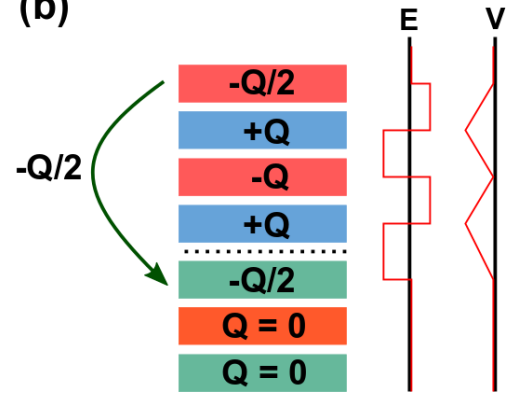

Figure 2.7.: Illustration of the polar catastrophe of two ionic crystals. Without reconstruction, the alternating polar layers yields the shown behavior of the electric field $E$ and the electrostatic potential $V$. When the charge $-Q / 2$ is transferred from the surface to the interface, the electric field oscillates around zero and the potential converges to a finite value. Reillustrated from [33].

cies and a structural relaxation [198, 199], which removes half of the oxygen atoms forming a zig-zag $(1 \times 2)$ reconstruction [199]. The dipole moment of the $\mathrm{MnO}_{2^{-}}$ terminated (001) surface of $\mathrm{LaMnO}_{3}$ is compensated by defect formation, i.e. by surface rumpling, or charge redistribution [200-202]. XMCD experiments indicated electron accumulation and thus the formation of $\mathrm{Mn}^{2+}$ at the $\mathrm{LaMnO}_{3} / \mathrm{SrTiO}_{3}(001)$ interface [44]. In contrast, nonpolar-terminated manganites, such as the (001) plane of $\mathrm{SrMnO}_{3}$ and $\mathrm{CaMnO}_{3}$, do not have to compensate for the polar discontinuity. Nonetheless, the symmetry-break at the surface leads to a partial occupation of the $e_{\mathrm{g}}$ orbital [203, 204. When considering a structurally unrelaxed $\mathrm{CaMnO}_{3}$ surface, the occupation leads to a spin-flip at the surface and thereby changes the antiferromagnetic order in the bulk to a ferromagnetic coupling [203, 205]. While the relaxed surface structure shows the same antiferromagnetic order as the bulk, introducing 0.04 electrons per manganese ion would induce the mentioned spin-flip and a ferromagnetic surface coupling [205].

Theoretical calculations of the (001) surface of mixed-valence manganites with a hole doping of $x=0.3$ indicate a charge transfer from the bulk to the surface resulting in the enrichment of $\mathrm{Mn}^{3+}$ at the surface (see Fig 2.8p) [39, 40]. The expected nominal $\mathrm{Mn}$ valence for a nonpolar surface would be $4+$. However, since the outermost $\mathrm{Mn}$ ions show an oxygen deficiency due to the removal of the oxygen ions at the surface, the surface $3 z^{2}-r^{2}$ orbital is energetically favored and the ionic state of the Mn ions becomes $3+$. Hence, the $3 z^{2}-r^{2}$-orbitals are almost fully occupied, while the $3 \mathrm{x}^{2}-\mathrm{y}^{2}$-orbitals are fully depleted. Therefore, the double-exchange mechanism is completely suppressed at the surface [39] and a nonconducting and antiferromagnetic surface layer is formed, i.e. a dead layer, which is restored within 3 u.c. 39, 40. As a consequence, the enrichment of $\mathrm{Mn}^{3+}$ ions and the symmetrybreaking lower the lattice symmetry to the low-symmetric orthorhombic (Pnma) 

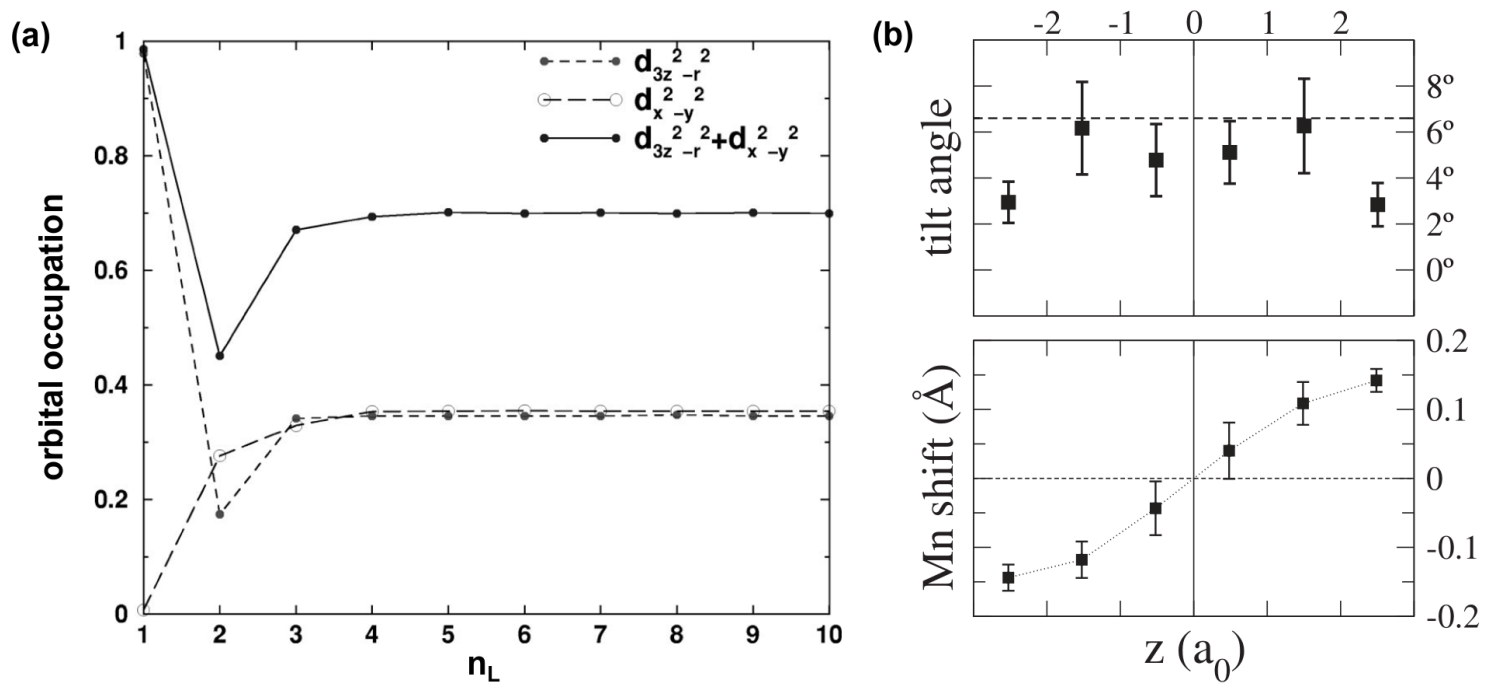

Figure 2.8.: (a) Occupation of the d-orbitals within the individual layers $n_{\mathrm{L}}$ of the surface slab with $n_{\mathrm{L}}=1$ as surface layer. The symmetry-breaking at the surface causes a charge redistribution, resulting in the enrichment of $\mathrm{Mn}^{3+}$ in the surface layer and a symmetry-lowering to the orthorhombic structure. The occupation of the $3 z^{2}-r^{2}$ is favored due to the lower Coulomb repulsion, which is induced by the missing oxygen ions at the surface. As a consequence, the $\mathrm{x}^{2}-\mathrm{y}^{2}$ orbital is depleted. (b) To accommodate the structural changes at the surface, strong surface rumpling occurs, shifting the Mn from its bulk position, here shown in units of the lattice constant $a_{0}=3.89 \AA$. The $z$ origin is defined at the center of the slab. From [39] and [206] with the kind permission of the American Physical Society.

structure [39, 206]. As shown in Fig 2.8p, the oxygen deficiency further induces a strong rumpling and tilting of the $\mathrm{MnO}_{6}$ octahedra [206], comparable to the structural changes in the parent compound $\mathrm{LaMnO}_{3}$ [201, 202. However, it has to be noted that the charge redistribution may depend on the surface termination of the manganite [40] and, most likely, may be different in the presence of defects, such as oxygen vacancies or dopant segregation. Photoemission [207, 208] and coaxial impact-collision ion scattering [209] measurements indicated a $\mathrm{MnO}_{2}$-terminated surface of $\mathrm{La}_{0.7} \mathrm{Ca} / \mathrm{Sr}_{0.3} \mathrm{MnO}_{3}$ thin films grown on $\mathrm{SrTiO}_{3}(100)$ and $\mathrm{LaAlO}_{3}(100)$ substrates. Furthermore, doping-dependent photoemission experiments indicated a change to the (LaCa/Sr)O termination with decreasing doping [207]. In thin films, however, the substrate termination has to be taken into account when determining the concluding layer of the manganite surface. Considering a manganite film grown on $\mathrm{TiO}_{2}$-terminated $\mathrm{SrTiO}_{3}$, the film surface ends with an $\mathrm{MnO}_{2}$ layer. In contrast to that, the manganite exhibits a ( $\mathrm{LaSr}$ )O surface layer, if the substrate terminates with a SrO layer [210, 211]. Yet, it has to be noted further, that the surface termi- 
nation is often determined under UHV conditions and may not correspond to the termination under ambient atmosphere. At ambient conditions, the accumulation of a native water layer cannot be prevented leading to the formation of $\mathrm{B}-\mathrm{OH}$ bonds. These bonds, in turn, can alter the surface structure and may further contribute to the compensation of the polar catastrophe [212].

Although BorcA et al. observed a change of the $\mathrm{Mn}$ valence in $\mathrm{La}_{0.7} \mathrm{~Pb}_{0.3} \mathrm{MnO}_{3}$, they attributed the presence of $\mathrm{Mn}^{3+}$ at the surface to the possibly higher oxidation states of the $\mathrm{Pb}$ ions [89], induced by the occurring chemical segregation in the films studied. These chemical segregation effects of the A-site cation as well as the formation of oxygen vacancies are a well-known problem in oxide films [3638, 87, 89, 213, 214]. While they might also compensate the polar discontinuity at the surface, they exhibit a serious obstacle for the practical application of correlated oxides. Both phenomena depend on the chosen growth conditions, the oxygen partial pressure in particular. Commonly used deposition techniques, such as pulsed-laser deposition and sputtering, often suffer from a low oxygen partial pressure giving rise to segregation and oxygen deficiency [85-87, 90, 183, 215 217]. Oxygen vacancies at the surface can cause the occupation of the $e_{\mathrm{g}}$-orbital in $\mathrm{SrMnO}_{3}$ and, depending on the amount of oxygen vacancies present at the surface [204], may induce a spin-flip at the surface. X-ray absorption spectroscopy (XAS) and photoemission experiments indicated that the presence of $\mathrm{Mn}^{2+}$ states at the surface of PLD-grown $\mathrm{La}_{0.7} \mathrm{Sr}_{0.3} \mathrm{MnO}_{3}$ [90, 91] and $\mathrm{La}_{0.7} \mathrm{Ca}_{0.3} \mathrm{MnO}_{3}$ [92, 93] films is linked to oxygen vacancy formation. While these effects are quite pronounced in as-deposited films kept in air, there is no indication for $\mathrm{Mn}^{2+}$ surface states in post-annealed samples and samples kept in vacuum all along [92]. Post-annealing presumably minimizes the amount of oxygen vacancies, whereas the vacuum prevents the abreaction of a not-fully oxidized film with, for example, $\mathrm{CO}_{2}$ residing in air. Furthermore, in-situ low-energy electron diffraction and scanning tunneling microscopy (STM) measurements revealed a $(\sqrt{2} \times \sqrt{2}) \mathrm{R} 45^{\circ}$ surface reconstruction [42, 218] in well oxidized films (i.e. a minimal amount of oxygen vacancies), which is caused by the octahedral tilting within the manganite system [218]. By artificially inducing oxygen vacancies, i.e. by applying a lower oxygen partial pressure during deposition, however, additional reflections appear suggesting a different reconstruction. Indeed, STM experiments showed the reversible transition between the $(\sqrt{2} \times \sqrt{2}) \mathrm{R} 45^{\circ}$ and $(1 \times 1)$ reconstruction by oxygen adsorption and desorption, respectively [218] (see Fig.2.9). Furthermore, the transition from a $(1 \times 1)$ to a $(\sqrt{2} \times \sqrt{2}) \mathrm{R} 45^{\circ}$ reconstruction changes the electronic state of the surface. While the $(\sqrt{2} \times \sqrt{2}) \mathrm{R} 45^{\circ}$ reconstruction yields insulating characteristics with a band gap of $1.35 \mathrm{eV}$, the $(1 \times 1)$ surface is metallic [218].

Surface segregation of the divalent doping element is driven by the minimization of the elastic energy and electrostatic interactions [183]. These minimization effects are intrinsically caused by doping due to the different cation sizes and extrinsically due to the accommodation of substrate-induced strain [219 221]. Along the sim- 

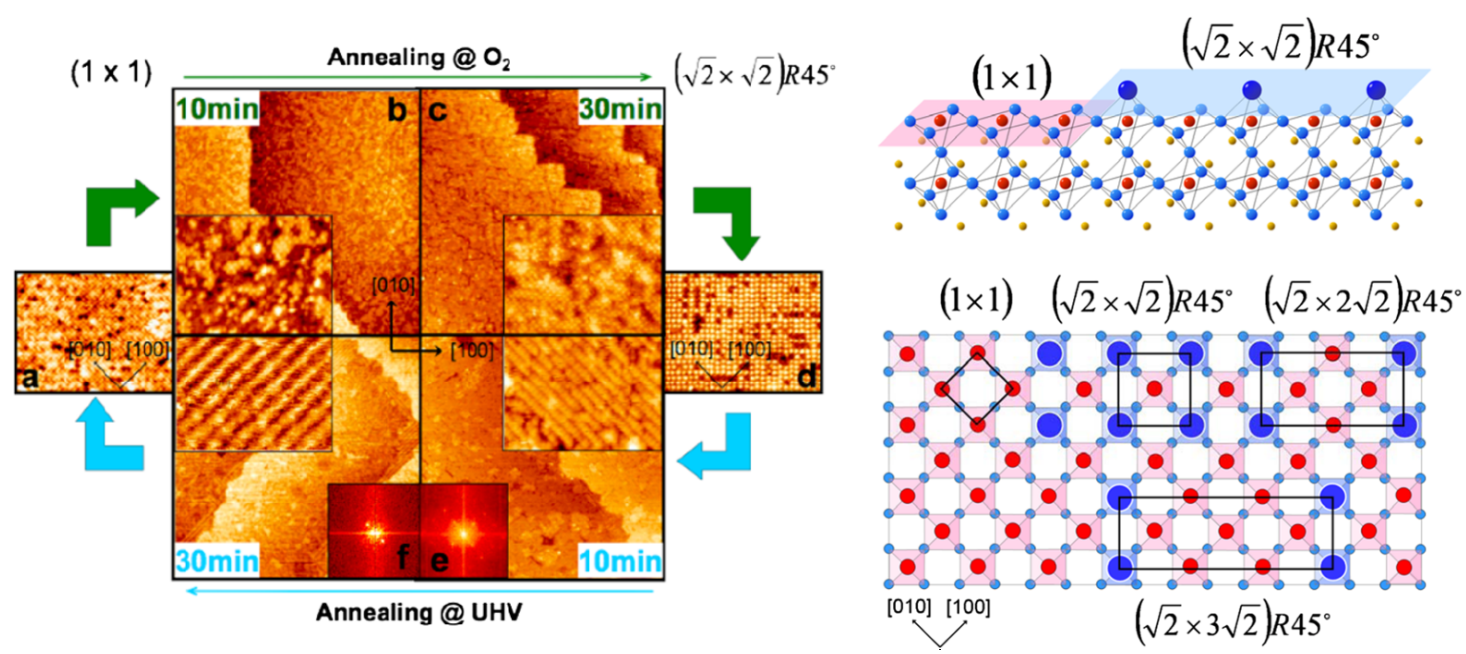

Figure 2.9.: Adsorption/desorption of oxygen at the surface induces a reversible transition between the insulating $(\sqrt{2} \times \sqrt{2}) \mathrm{R} 45^{\circ}$ and metallic $(1 \times 1)$ surface. While adsorbing, the surface traverses different metastable surface reconstructions, namely $(\sqrt{2} \times 2 \sqrt{2}) \mathrm{R} 45^{\circ}$ and $(\sqrt{2} \times 3 \sqrt{2}) \mathrm{R} 45^{\circ}$. From [218] with the kind permission of the American Physical Society.

ple enrichment of the dopant at the surface [36, 38, 89], segregation may result in a structural reconstruction. Angle-resolved x-ray photoelectronen spectroscopy on $\mathrm{La}_{0.7} \mathrm{Sr}_{0.3} \mathrm{MnO}_{3}$ [36, 37] and $\mathrm{La}_{0.7} \mathrm{~Pb}_{0.3} \mathrm{MnO}_{3}$ [38, 89] indicated a Ruddlesden-Popperlike surface layer with a stoichiometric composition of $\left(\mathrm{La}_{0.67}(\mathrm{Sr}, \mathrm{Pb})_{0.33}\right)_{2} \mathrm{MnO}_{4}$. In addition, x-ray photoemission spectroscopy experiments revealed the formation of monolayer thick $\mathrm{SrO}$ [213] and $\mathrm{SrCO}_{3}$ [87] surface layer, respectively. Therefore, segregation effects represent a serious obstacle for feasible surface studies and should always be taken into account in the interpretation of surface sensitive experiments.

Considering the interface, the most important difference to the surface is the presence of the substrate and the resulting substrate-induced biaxial strain imposed on the film. The mismatch between film and substrate induces a change of the bond length and bond angles and, therefore, the hopping element $t_{\text {eff. Consequently, the }}$ applied strain will have a huge impact on the electrical and magnetic properties of the film, e.g. changes of the transition temperature $T_{\mathrm{C}}[222+225]$. Strain further lifts the degeneracy of the $e_{\mathrm{g}}$ and $t_{2 \mathrm{~g}}$ orbitals, similar to the Jahn-Teller effect. Since the $t_{2 \mathrm{~g}}$ electrons represent a localized rump spin, the lifting does not change the measurable properties of the manganites and can, therefore, be neglected. The raised degeneracy further depends on the direction of the applied strain. While tensile strain stretches the Mn-O bonds, a compressive strain squeezes the Mn-O bonds in the $a b$-plane and elongates the bonds in $c$-direction. Accordingly, the elongation/compression of the $\mathrm{Mn}-\mathrm{O}$ bonds in the $a b$-plane results in a lower/stronger Coulomb repulsion and, thus, in favoring the $\mathrm{x}^{2}-\mathrm{y}^{2}$ (tensile strain) or $3 \mathrm{z}^{2}-\mathrm{r}^{2}$ (compressive strain) orbital [226]. X-ray linear dichroism (XLD) and photoemission ex- 
periments indicated that such a relation may not apply in ultrathin films with a thickness of $d \leq 6$ u.c. [227-230]. TEBANo et al. and CUI et al. showed by means of XLD that regardless of the expected orbital polarization, imposed by the applied strain, ultrathin films always feature the occupation of the $3 \mathrm{z}^{2}-\mathrm{r}^{2}$ orbital [227, 230]. However, HuiJBen et al. observed the expected $\mathrm{x}^{2}-\mathrm{y}^{2}$-orbital ordering down to 3 u.c. without any indication of the occupation of the $3 z^{2}-r^{2}$-orbital [189]. XLD experiments by PESQUERA et al. attributed the $3 \mathrm{z}^{2}-\mathrm{r}^{2}$ orbital occupation to the free surface of the $\mathrm{La}_{0.7} \mathrm{Sr}_{0.3} \mathrm{MnO}_{3}$ film, which would be predominant in ultrathin films of only a few monolayers [211]. Furthermore, they were able to tune the orbital polarization of $\mathrm{La}_{0.7} \mathrm{Sr}_{0.3} \mathrm{MnO}_{3}$ by tailoring its surface termination. As mentioned before, a change of the substrate termination alters the stacking of the AO- and $\mathrm{BO}_{2}$-layers of the manganite and, thus, the terminating surface layer. $\mathrm{A} \mathrm{MnO}_{2}$ terminated surface induces a higher polarization of the $3 \mathrm{z}^{2}-\mathrm{r}^{2}$-orbital, as expected from ab-initio calculation [39], whereas a (LaSr)O termination leads to its depletion. This circumstance may further explain the observed discrepancies of previous XLD and XAS measurements [189, 227, 228, 230]. The orbital polarization has also an impact on the electrical and magnetic properties at the interface, since the magnetic coupling is either promoted in the in-plane or out-of-plane direction. Consequently, the formation of an A-type or C-type antiferromagnet at the interface is favored [228. The antiferromagnetic coupling competes with the ferromagnetic order in the remaining film and contributes to the suppression of the magnetization at the interface this way. Figure 2.10 illustrates the orbital occupation and the corresponding antiferromagnetic coupling.

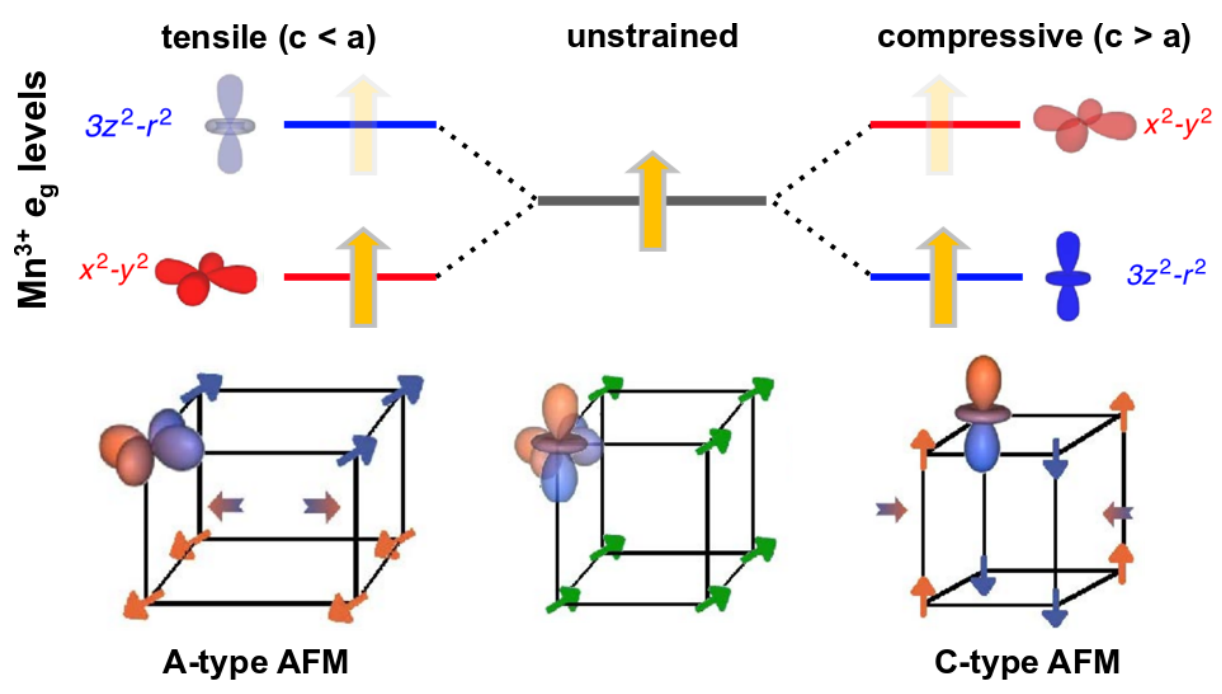

Figure 2.10.: Tensile strain promotes the occupation of the $\mathrm{x}^{2}-\mathrm{y}^{2}$ orbital, whereas the electron occupies the $3 \mathrm{z}^{2}-\mathrm{r}^{2}$ orbital in compressively strained films. This results either in an A-type or a C-type antiferromagnetic coupling at the interface. Reillustrated from [211, 227]. 
In addition to a simple change of the bond lengths, the epitaxial strain can also be realized by a different connectivity of the octahedral network [231 233], which is nicely demonstrated by ultrathin $\mathrm{La}_{0.7} \mathrm{Sr}_{0.3} \mathrm{MnO}_{3}$ films grown on cubic $\left(\mathrm{LaAlO}_{3}\right)_{0.3}\left(\mathrm{Sr}_{2} \mathrm{AlTaO}_{6}\right)(100)$ (LSAT) and orthorhombic $\mathrm{NdGaO}_{3}(110)$ (NGO) substrates [234], both substrates having a similar pseudo-cubic lattice constant, $a_{\mathrm{LSAT}}$ $=3.868 \AA$ and $a_{\mathrm{NGO}}=3.862 \AA$, respectively. However, they will induce a different tilting of the octahedra, i.e. a different octahedral connectivity, due to their differing lattice structure. Such diverging connectivity results in a significant decrease of $T_{\mathrm{C}}$ by $140 \mathrm{~K}$ for films grown on NGO substrates [234]. Because of the perturbed octahedral connectivity within the film, the overlap of the Mn d-orbitals and the oxygen p-orbitals changes, which leads to a weakening of the double-exchange mechanism. However, introducing a 9 u.c. thick buffer layer of a cubic material (e.g. $\mathrm{SrTiO}_{3}$ ) can enhance the connectivity between film and substrate. The cubic buffer layer imposes a straightening on the out-of-plane bond angles, which shifts the bond angle closer to its bulk value. This increases the hopping element $t_{\text {eff }}$ and in this way, the transition temperature in the films grown on buffered NGO is increased by $100 \mathrm{~K}[225$. However, when the buffer layer becomes too thick $(d>10$ u.c.) the electrical and magnetic properties of the manganite film are more and more governed by the tensile strain of the $\mathrm{SrTiO}_{3}$. The mechanism of the octahedral tilting to compensate for the strain- and symmetry-induced structural changes at the interface is comparable to the strong rumpling, predicted and observed for the surface [201, 202, 206, 235]. Additionally to the aforementioned octahedral connectivity, interdiffusion between film and substrate [235] and charge redistribution [42, 44, 46, 236 238] may also play a role in balance out the polar discontinuity, similar to the segregation effects and electron accumulation at the surface.

At the $\mathrm{La}_{0.7} \mathrm{Sr}_{0.3} \mathrm{MnO}_{3} / \mathrm{SrTiO}_{3}$ interface, charge variations [236 238 and electron delocalization [45, 46] between the $\mathrm{e}_{\mathrm{g}}$ orbitals of $\mathrm{Mn}$ and the empty $3 \mathrm{z}^{2}-\mathrm{r}^{2}$ orbitals of Ti cause antiferromagnetic coupling between $\mathrm{Mn}$ and Ti [45, 239]. In contrast to the electron delocalization between substrate and film, XMCD measurements indicated an enrichment of $\mathrm{Mn}^{3+}$ at the $\mathrm{La}_{0.7} \mathrm{Sr}_{0.3} \mathrm{MnO}_{3} / \mathrm{SrTiO}_{3}$ interface [41-43]. The electron-enriched interface region reaches a thickness up to 8 u.c., which corresponds well to the thickness of the electron-enriched surface layer determined by c-AFM [187, 240], XMCD [93, 102] and XMRS [103, 104]. However, negative charge accumulated at the interface has to be compensated by the same amount of positive charge transported away from the interface to achieve charge neutrality. Assuming a fixed stoichiometry, a likely reaction of the system is to create more $\mathrm{Mn}$ ions with a higher oxidation state, i.e. $\mathrm{Mn}^{4+}$ in $\mathrm{La}_{0.7} \mathrm{Sr}_{0.3} \mathrm{MnO}_{3}$, or oxygen vacancies, where recent photoemission and LEED measurements support the latter [42]. Similar to the bulk and surface, the interface is electronically phase separated and shows percolative behavior. However, at this point, scanning SQUID microscopy measurements on 4 u.c. thin $\mathrm{LaMnO}_{3}$ films on STO should be mentioned [241]. In contrast to the known electronic phase separation in the bulk [155] and at the surface [192], meaning nanometer-sized ferromagnetic domains in an antiferromagnetic matrix, 
scanning SQUID microscopy revealed a superparamagnetic phase instead of ferromagnetic domains. This was attributed to electronic reconstructions at the interface due to the polar discontinuity.

As the discussed experimental results show, the polar discontinuity is generally compensated by charge accumulation. However, the exact mechanism (e.g. formation of oxygen vacancies, enrichment of $\mathrm{Mn}^{2+/ 3+}$ ) depends strongly on the growth conditions. To conclude the description of the surface and interface properties of the mixed-valence manganites, recent angular-dependent XLD experiments on ferromagnetic $\mathrm{La}_{0.67} \mathrm{Sr}_{0.33} \mathrm{MnO}_{3}$ should be mentioned. These experiments revealed an antiferromagnetic order at the surface, which is coupled non-collinear to the ferromagnetic bulk structure. Such a non-collinear coupling was traced back to a large spin-orbit coupling at the surface [242], in contrast to previous observations, which implied a relatively low spin-orbit coupling in manganites. Such a large coupling could result in non-trivial and exotic spin textures at the surface/interface, such as skyrmions [186, 243], and may open new possible applications of manganites beyond the currently discussed magnetoresistive devices and electrode material in catalytic reactions.

\subsection{Raman scattering}

When photons are scattered by condensed matter, most of them are elastically scattered, so that the incident and the scattered photons have the same energy. However, a small fraction of photons is inelastically scattered. These photons possess a different energy as the incident photons and are known as Raman scattered photons. Each excitation, which can be probed by Raman spectroscopy, has a specific spectral characteristic, whether it is represented by a distinct or by a broad feature in the spectrum, e.g. a background continuum. A schematic spectrum consisting of typical Raman-active excitations is shown in Fig.2.11.

In the following, a brief description of the classical phononic Raman process will be given, based on the Refs. [244 246]. In the simplest case, the medium is perturbed by an external electric field $\vec{E}$ causing the induced macroscopic polarization

$$
\vec{P}=\epsilon_{0} \chi \vec{E}(t)
$$

with $\chi$ as electrical susceptibility, which is, in general, a second-rank tensor. The external electromagnetic field can be expressed as $\vec{E}_{0}=\vec{E}_{0} \mathrm{e}^{i(\vec{k} \cdot \vec{r}-\omega t)}$, with the frequency $\omega$ and the wave vector $\vec{k}$. $\vec{u}$ further represents a displacement of an atom from its average position, which in harmonic approximation can be expressed as $\vec{u}(\omega, \vec{q})=\overrightarrow{u_{0}} \cos \left(\vec{q} \cdot \vec{r}-\omega_{k} t\right)$. These displacements are small compared to the lattice constants and describe collective lattice waves with frequency $\omega_{k}$ and wave vector $\vec{q}$. They represent independent vibrational modes of the lattice, the normal modes. In 


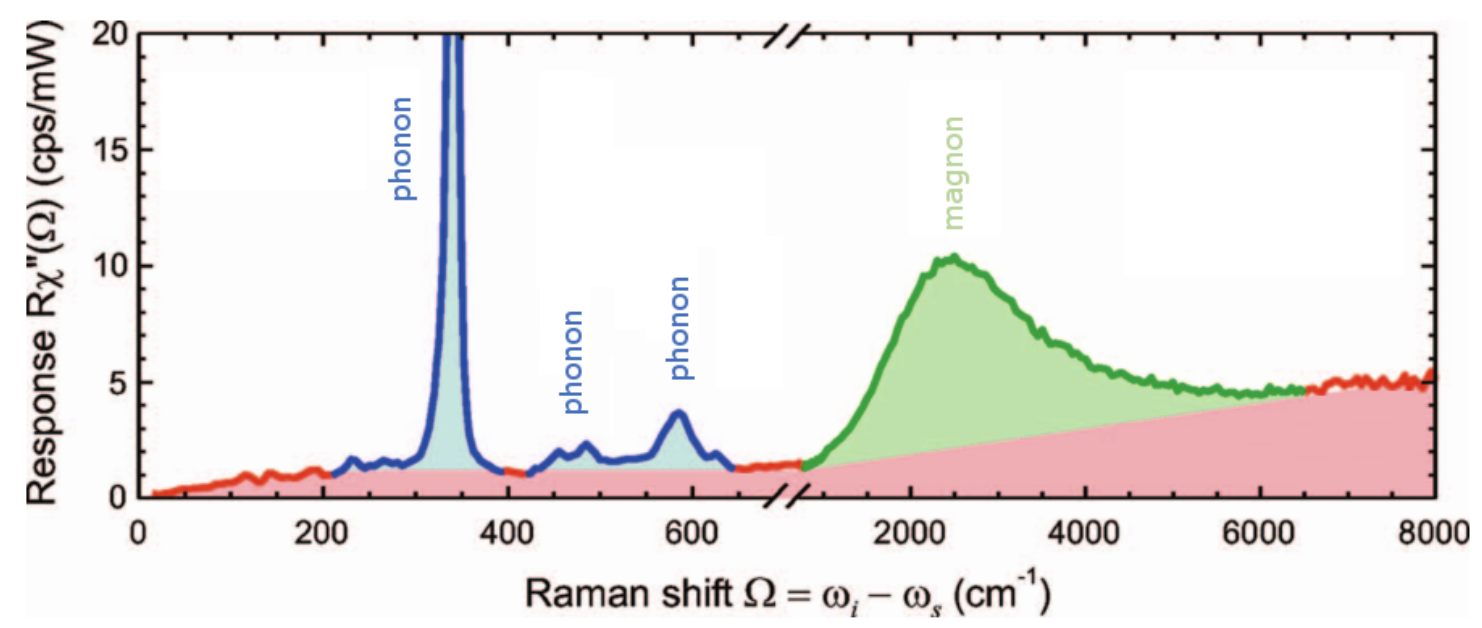

Figure 2.11.: An exemplary Raman spectrum of a crystalline sample. Each excitation is marked in a different color. Phonons are usually located below $1000 \mathrm{~cm}^{-1}$, whereas magnons reside in higher wave number regions (green). Electronic excitations (red) are underlying the whole spectrum. Taken from [57] with the kind permission of the American Physical Society.

the Born-Oppenheimer approximation, the electrons follow the motion of the atoms instantaneously. As a consequence, $\chi$ can be expressed as a function of $\vec{u}$. A Taylor expansion of $\chi$ for lattice vibrations with $\vec{u}_{0}$ yields

$$
\chi(\vec{u})=\chi_{0}+\left(\frac{\partial \chi}{\partial u}\right) \vec{u}+\mathcal{O}\left(n^{2}\right)
$$

While $\chi_{0}$ corresponds to the susceptibility without vibrational contribution, the linear term describes the periodic modulation of $\chi$ by the lattice displacement, i.e a first order Raman process. Subsequent terms correspond to Raman processes of higher order involving two or more lattice vibrations. Inserting Eq. (2.3) into the polarization $\vec{P}$ given in Eq. 2.2$)$ while neglecting terms of higher order leads to

$$
\vec{P}=\epsilon_{0} \chi_{0} \vec{E}(t)+\epsilon_{0}\left(\frac{\partial \chi}{\partial u}\right) \vec{u} \vec{E}(t)
$$

The static term $\chi_{0} \vec{E}(t)$ expresses oscillations of the macroscopic polarization, which are in phase with the incident radiation. This is basically the elastically scattered radiation, the Rayleigh scattering. Therefore, the inelastic scattering is expressed by

$$
\vec{P}_{R S}=\epsilon_{0}\left(\frac{\partial \chi}{\partial \vec{u}}\right) \vec{u} \vec{E}(t)
$$


Inserting $\vec{E}$ and $\vec{u}$ into Eq. 2.5 results in an expression for $\vec{P}$, which contains frequencies and wave numbers shifted with respect to the incident radiation,

$$
\vec{P}_{i n d}=\frac{1}{2}\left(\frac{\partial \chi}{\partial \vec{u}}\right) \epsilon_{0} \overrightarrow{u_{0}} \vec{E}_{0}\left[\cos \left(\left(\omega+\omega_{k}\right) t\right)+\cos \left(\left(\omega-\omega_{k}\right) t\right)\right]
$$

There are two possible processes, which can occur: the annihilation or the creation of a normal mode excitation (i.e. a phonon). The annihilation of a normal mode increases the frequency with respect to the incident radiation (Anti-Stokes process), whereas the creation of the normal mode will decrease the frequency (Stokes process). In general, the modulation of the susceptibility by the displacement is described by the Raman tensor

$$
\left(R_{i j}\right)_{k}=\left(\frac{\partial \chi_{i j}}{\partial u}\right)
$$

This second-rank tensor reflecting the symmetry of the lattice distortions. It can be shown that the differential Raman scattering cross section, i.e. the probability of a photon to be inelastically scattered into the solid angle $d \Omega$ with an energy between $\omega$ and $\omega+\mathrm{d} \omega$, is

$$
\frac{\mathrm{d} \sigma}{\mathrm{d} \Omega} \sim\left(\omega \pm \omega_{k}\right)^{4}\left|\overrightarrow{e_{s}} R_{k} \overrightarrow{e_{i}}\right|^{2}
$$

Therefore, the intensity of a Raman scattered process is given by

$$
I_{R S} \sim\left|\overrightarrow{e_{s}} R_{k} \overrightarrow{e_{i}}\right|^{2}
$$

As it becomes apparent in Eq. 2.9), the intensity of a Raman process depends on the polarization of the incident and scattered radiation as well as the symmetry of the Raman tensor. This is summarized in the so-called Raman selection rules, which can be used to study particular normal modes through a Raman polarization analysis. In practice, the light's linear polarization is chosen with respect to the sample orientation and the analyzer selects specific contributions of the scattered light determining the observed normal modes in the measured Raman spectrum.

\subsubsection{Quantum mechanical description of Raman scattering}

While the classical framework of Raman scattering is sufficient enough to describe most of the occurring phenomena, it fails to appropriately describe resonant Raman scattering, magnon scattering and electron-phonon or phonon-phonon interactions [244, 245]. These phenomena require a quantum mechanical treatment of the Raman effect, e.g. by third-order perturbation theory. Details can be found in Ref. [245] or in most of the standard text-books about Raman scattering.

For the sake of completeness, a brief summary of the quantum mechanical de- 

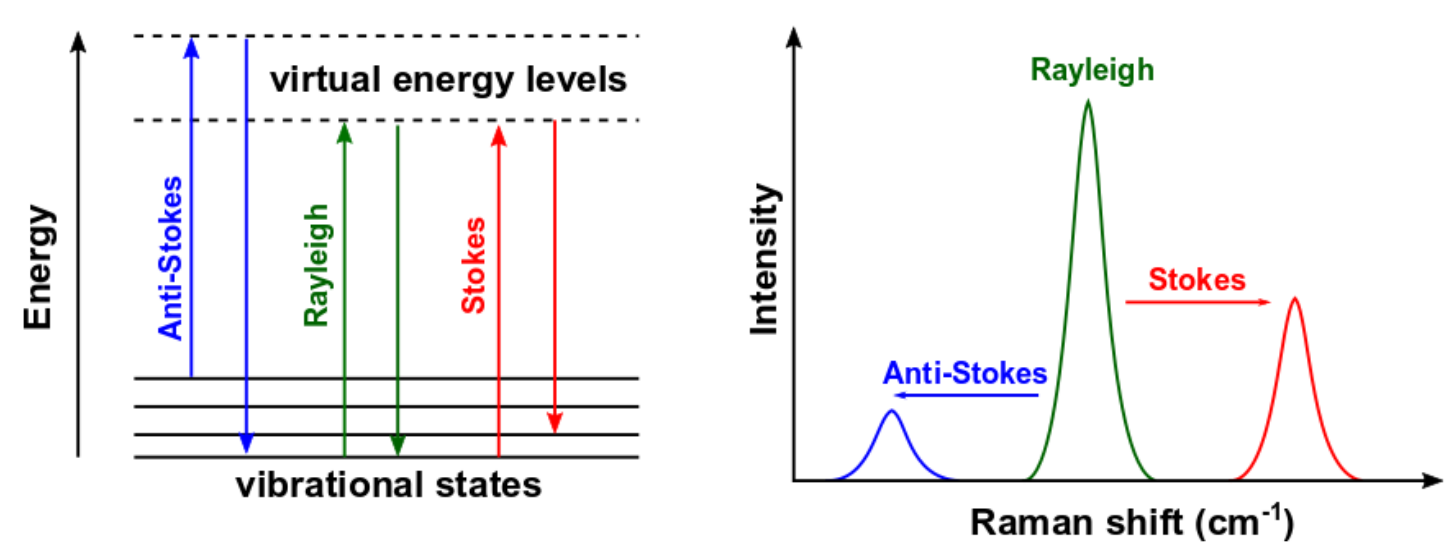

Figure 2.12.: Schematic of the different light scattering processes in a crystal or molecule: Rayleigh (green), Stokes (red) and Anti-Stokes (blue). While the inelastically scattered photons can have a higher (Anti-Stokes) or lower (Stokes) energy, the energy of the elastically (Rayleigh) scattered light remains the same. The different inelastic light scattering processes are reflected by a blue or red shift with respect to the incident light.

scription is given in the following. In this framework, an excited lattice vibration, i.e. a phonon, is a quantized quantity and, thus, the excited state has to be a multiple of the vibrational energy $\hbar \omega_{k}$. Figure 2.12 depicts the typical processes occurring throughout the excitation. In thermal equilibrium, the excited vibrational states are described by the Boltzmann distribution. As one can see in Fig.2.12, the Anti-Stokes process requires an already excited state, in which the studied analyte resides. Therefore, the probability for a Anti-Stokes process to occur is much smaller than that for a Stokes process and, as a consequence, Anti-Stokes lines have a much smaller intensity. The difference between both processes, i.e. Stokes and Anti-Stokes, is proportional to the Boltzmann distribution,

$$
\frac{I_{a s}}{I_{s}} \sim \exp \left[-\frac{\hbar \omega_{k}}{k_{B} T}\right]
$$

In addition to energy conservation, the momentum has to be conserved as well. In the visible region, the incident wave vector $k_{i}$ is of the order of $10^{5} \mathrm{~cm}^{-1}$ [244]. Since the size of the first Brillouin zone lies in the order of $10^{8} \mathrm{~cm}^{-1}$, a one-photon process can only occur when the scattering vector $q \approx 0$. Hence, only zone-centered phonons can be excited in a first order Raman process. Furthermore, the quantum mechanical treatment can explain the finite phonon lifetime $\tau$ and the phononic coupling to the electronic background continuum resulting in an asymmetric phonon lineshape present as Fano profiles [247]. The scattering cross section can be expressed further 
in terms of the dynamical structure factor $\mathrm{S}(\vec{q}, \omega)$, which is related to the imaginary part of the density-density response function [248]. With $\chi^{\prime \prime}$ as the imaginary part of the Raman response function and the Bose factor $n=1 /\left(e^{\left(\frac{\hbar \omega}{k_{\mathrm{B}} T}\right)}-1\right)$, the structure factor can be written as

$$
S(\vec{q}, \omega)=-\frac{\hbar}{\pi}[n+1] \chi^{\prime \prime}(\vec{q}, \omega)
$$

and can often be approximated by a collision-limited model [249]. Such an electronic background continuum was found to be an important probe in superconducting materials [250-252].

Within the quantum mechanical framework it is possible to explain the different intensities of the Stokes and the Anti-Stokes processes, the strong enhancement of a resonant Raman process as well as the asymmetric phonon lineshapes. However, for the interpretation and discussion of most of the experiments and phenomena observed in the scope of this thesis, the classical description is sufficient. Surfaceenhanced effects thereby play a separate role, since the quantum mechanical description of the surface-enhanced Raman scattering requires a fully quantum mechanical framework of the plasmonic enhancement, which has not yet been developed. In the recent years, different models were suggested to characterize the plasmonic enhancement as Raman processes of third or fourth order [253] or within quantum electrodynamics [254]. Therefore, the mechanisms of surface-enhanced Raman scattering in Ch 2.3 are described in a classical framework.

\subsubsection{Normal mode symmetries and selection rules of manganites}

A classification of lattice vibrations is given within the framework of group theory [244, 255]. Each normal mode is represented by a set of displacements, forming the basis for an irreducible representation in the character table of the corresponding point group. The character tables for each point group can be found in the International Tables of Crystallography [256]. Usually, the irreducible representations are denoted by the so-called Mulliken symbols [255]. The Mulliken symbols relevant for this thesis are explained in Tab2.1. An existing set of normal modes can then be divided into three classes: 1) Raman active, 2) infrared (IR) active and 3) silent modes. In crystal structures containing an inversion center, Raman-allowed modes are even $\left(R_{i j}=R_{j i}\right)$, whereas odd modes $\left(R_{i j} \neq R_{j i}\right)$ are Raman-forbidden. This is opposed to IR modes and is often referred to the mutual exclusion principle. A detailed explanation for the derivation of the irreducible representation is given by BERNARDO et al. [257]. The selection rules of a given Raman mode arise from the Raman tensor entries of the corresponding representation. In the following, the two most relevant space groups of the mixed-valence manganites, R $\overline{3} \mathrm{c}$ (No.167) and Pnma (No.62), and their corresponding Raman tensors will be discussed. 
Table 2.1.: The Mulliken symbols for the irreducible representations. In standard convention the $z$-axis is the principal axis of the system.

\begin{tabular}{c|l}
\hline A/B & $\begin{array}{l}\text { symmetric/antisymmetric with respect to } 90^{\circ} \text { rotation around the } \\
\text { principal axis } \\
\text { double degenerated mode } \\
\text { E }\end{array}$ \\
summetric/antisymmetric with respect to a vertical mirror \\
subscript g/u perpendicular to the principal axis \\
symmetric/antisymmetric with respect to inversion (even/odd \\
mode)
\end{tabular}

As mentioned in Ch 2.1, the ideal cubic perovskite structure can be converted into the rhombohedral or orthorhombic structure by tilting the $\mathrm{MnO}_{6}$ octahedron. The transition into the rhombohedral $(\mathrm{R} \overline{\mathrm{c}} \mathrm{c})$ structure causes a bisection of the Brillouin zone and, thus, the modes of the $\mathrm{R}\left(\frac{1}{2}, \frac{1}{2}, \frac{1}{2}\right)$ corner point of the Brillouin zone fall into its center [62]. Therefore, the rhombohedral structure features a set of 20 normal modes, five of which are Raman-allowed:

$$
\Gamma(\mathrm{R} \overline{3} \mathrm{c})=1 A_{1 \mathrm{~g}}+4 E_{\mathrm{g}}
$$

The Raman tensors of the $\mathrm{R} \overline{3} \mathrm{c}$ space group are:

$$
\begin{gathered}
\underline{\underline{R}}\left(A_{1 \mathrm{~g}}\right)=\left[\begin{array}{lll}
a & 0 & 0 \\
0 & a & 0 \\
0 & 0 & b
\end{array}\right] \quad \underline{\underline{R}}\left(E_{\mathrm{g}, 1}\right)=\left[\begin{array}{ccc}
c & 0 & 0 \\
0 & -c & d \\
0 & d & 0
\end{array}\right] \\
\underline{\underline{R}}\left(E_{\mathrm{g}, 2}\right)=\left[\begin{array}{ccc}
0 & -c & -d \\
-c & 0 & 0 \\
-d & 0 & 0
\end{array}\right]
\end{gathered}
$$

where $a, b, c$ and $d$ represent the non-zero tensor entries of the Raman tensor. A similar process occurs by lowering the symmetry further to the orthorhombic structure. In that case, the $\mathrm{R}\left(\frac{1}{2}, 0, \frac{1}{2}\right)$ and $\mathrm{R}\left(0, \frac{1}{2}, 0\right)$ points fall into the zone center [62]. This gives rise to 60 phonon modes, 24 of which are Raman active:

$$
\Gamma(\text { Pnma })=7 A_{1 \mathrm{~g}}+5 B_{1 \mathrm{~g}}+7 B_{2 \mathrm{~g}}+5 B_{3 \mathrm{~g}}
$$

Their corresponding Raman tensors are given by:

$$
\underline{\underline{R}}\left(A_{1 \mathrm{~g}}\right)=\left[\begin{array}{lll}
a & 0 & 0 \\
0 & b & 0 \\
0 & 0 & c
\end{array}\right] \quad \underline{\underline{R}}\left(B_{1 \mathrm{~g}}\right)=\left[\begin{array}{lll}
0 & d & 0 \\
d & 0 & 0 \\
0 & 0 & 0
\end{array}\right]
$$




$$
\underline{\underline{R}}\left(B_{2 \mathrm{~g}}\right)=\left[\begin{array}{ccc}
0 & 0 & e \\
0 & 0 & 0 \\
e & 0 & 0
\end{array}\right] \quad \underline{\underline{R}}\left(B_{3 \mathrm{~g}}\right)=\left[\begin{array}{lll}
0 & 0 & 0 \\
0 & 0 & f \\
0 & f & 0
\end{array}\right]
$$

ILIEV et al. and ABRASHEV et al. performed prototypical lattice dynamical calculations based on a shell model and compared them to experimental spectra [58, 258]. The vibrational modes can be attributed mainly to stretching and bending vibrations of the oxygen octahedra and, occasionally, to the movement of the A-site cations. However, these vibrations are usually found below $200 \mathrm{~cm}^{-1}$ [58]. The spectral region between $200 \mathrm{~cm}^{-1}$ and $400 \mathrm{~cm}^{-1}$ is dominated by rotational modes of the $\mathrm{MnO}_{6}$ octahedra. These modes are directly linked to the tilt angle of the octahedra [58, 59, 259] and depend strongly on external perturbations, such as temperature [61, 260, 261] or pressure, as well as on intrinsic perturbations [262], like the A-site cation [59], isotope effects [263], substrate-induced strain [264 266] or doping [267]. While the low- and mid-frequency region is mainly influenced by the lattice, the high frequency region, $\nu=400-650 \mathrm{~cm}^{-1}$, is affected by the metal-insulator transition and the Jahn-Teller distortion within the manganite system [58, 59, 65, 66, 268]. Figure 2.13 illustrates the Raman-active modes of interest for this thesis. Since this thesis addresses primarily the polaronic behavior probed by Raman spectroscopy, a more detailed discussion of the corresponding bending and stretching modes modes and their behavior will be given in $\mathrm{Ch} 2.4$.

(a)

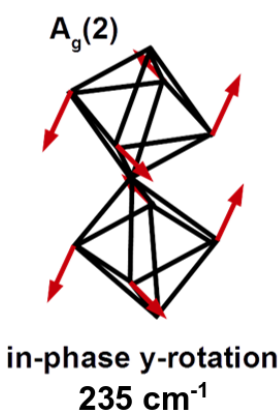

(b)

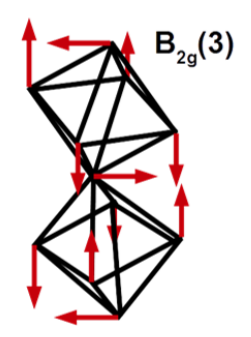

out-of-phase bending $440 \mathrm{~cm}^{-1}$ (c)

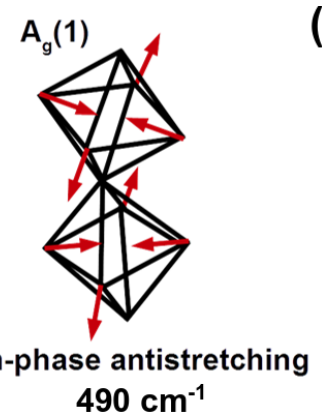

(d) $B_{2 g}(1)$

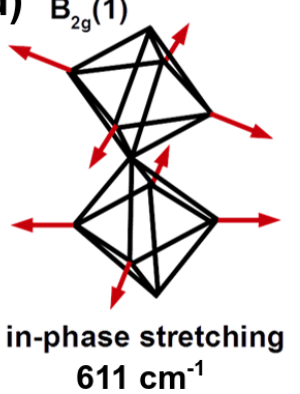

Figure 2.13.: Illustration of the Raman modes of interest: a rotational mode at $235 \mathrm{~cm}^{-1}$ (a), the bending mode at $440 \mathrm{~cm}^{-1}$ (b) and the Jahn-Teller stretching vibrations at $490 \mathrm{~cm}^{-1}$ (c) and $611 \mathrm{~cm}^{-1}$ (d). Adapted from [58].

According to their irreducible representations (see Eq.(2.12) and (2.15), both structures have a different number of Raman-allowed normal modes and different contributing symmetries. Therefore, both manganite structures can be distinguished by their number of visible normal modes as well as their selection rules. Consequently, the individual Raman spectrum can be denoted as a fingerprint of the studied system, which allows a precise identification of a certain structure or distortion within the crystal structure, e.g. orthorhombic and rhombohedral. Moreover, more advanced methods, such as tip-enhanced Raman scattering, facilitate, in principle, the structural characterization of regions in the nanometer range [69, 70]. 


\subsubsection{Raman instrumentation}

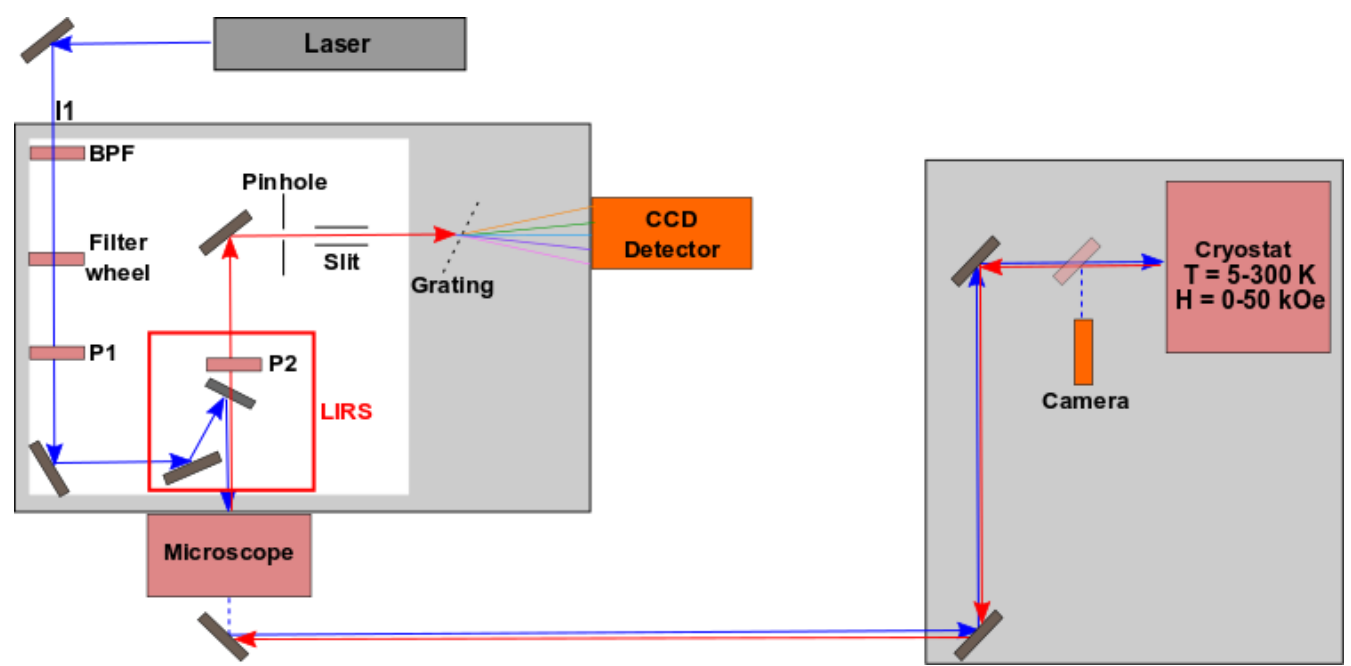

Figure 2.14.: A schematic of the optical path in the used Raman spectrometer.

In this thesis, a Horiba Jobin Yvon LabRAM HR Evolution spectrometer [269] with an integrated confocal micro Raman spectrometer and a microscope coupled to a $800 \mathrm{~mm}$ focal length spectrograph was used. A schematic of the optical path is shown in Fig 2.14. The linearly polarized laser light $(532 \mathrm{~nm}$ or $632.8 \mathrm{~nm})$ enters the optics at iris I1, where a bandpass filter (BPF) suppresses any additional laser lines that may be present in the light. Subsequently, the light passes a filter wheel, equipped with filters of different opacity, where the laser intensity is attenuated to a certain degree. At the laser-injection-rejection system (LIRS), an edge filter reflects the beam towards the microscope, where it is focused onto the sample. The microscope is equipped with a motorized microscope stage (Märzhäuser Scan System) enabling Raman mapping and imaging. The backscattered light passes the LIRS, where only the inelastically scattered light is transmitted when passing through the confocal pinhole and the slit into the spectrograph. Optical elements such as polarizer (P1) and analyzer (P2) can be inserted into the optical path between the LIRS and the confocal hole. Within the spectrograph, the beam is collimated by a spherical mirror and is then reflected onto a switchable optical grating $(600 \mathrm{gr} / \mathrm{mm}$ or $1800 \mathrm{gr} / \mathrm{mm}$ ). The diffracted light is collected by another mirror and focused onto a CCD camera. Temperature- and magnetic-field-dependent measurements in a temperature range of $T=5-300 \mathrm{~K}$ and applied magnetic fields of $H=0-50 \mathrm{kOe}$, can be performed by means of a Microstat MO cryostat (Oxyford Instruments) [270]. For this, the laser beam is guided from the integrated Raman microscope to a similar self-build microscope by two mirrors. Then, it is again focused on the sample within the cryostat and, finally, the backscattered light is collected by the spectrometer. Typically, the Raman spectrum is illustrated as an intensity vs. Raman shift plot, where the Raman shift describes the difference between the wavelength of the incident and of the scattered light. As a result, the Raman spectra 
are wavelength-independent. Since the inelastic scattered light always consists of Stokes and Anti-Stokes scattering, the spectrum is mirrored at the abscissa, but the intensity of the Anti-Stokes scattering is much lower due to the aforementioned thermally excited state (see Ch.2.2.1). However, as a consequence of the used edge filter, the Raman setup used in this thesis is not capable to measure Anti-Stokes scattering.

\subsection{Surface-enhanced Raman scattering}

The small Raman scattering cross section, typically of the order of $10^{-30} \mathrm{~cm}^{2}$ and below [246], limits the sensitivity of Raman scattering for the characterization of ultrathin films and tiny amounts of molecules. This poor Raman cross section can be enhanced by exploiting the excitation of surface plasmons in metallic nanostructures. The first observations of surface-enhanced Raman scattering were made by FLEISCHMANN et al. on pyridine molecules in aqueous solution on a roughened silver electrode [271]. However, they attributed the enhanced intensity to the increase of the electrode surface due to the roughening procedure. VAN DUYNE et al. [272] and, independently, Creighton et al. [273] recognized that the high intensity obtained from Raman modes of pyridine has to be a true enhancement of the Raman scattering efficiency and called this phenomena surface-enhanced Raman scattering. In the following chapter, the two mechanisms underlying this huge intensity enhancement, the electromagnetic enhancement (EM) and the chemical enhancement (CE), are briefly summarized.

\subsubsection{Electromagnetic enhancement}

As a consequence of the reduced dimensionality of the metallic nanostructures, their absorption is dominated by the collective oscillation of the conduction electrons. Therefore, incident monochromatic light excites these collective oscillations, which are known as surface plasmon polaritons (SPP) [274, 275]. At this juncture, the term plasmon refers to a general oscillation of the plasma in metals and the term polariton to a plasma oscillation, which is excited by an electromagnetic wave. Since the excited plasmons in bulk structures, planar metal-dielectric interfaces and nanoparticles differ from each other due to their different boundary conditions, they have to be addressed separately. This thesis mainly utilizes the excitation of surface plasmon polaritons in metallic nanoparticles. In these structures, the SPP is also known as localized surface plasmon resonance (LSPR) [274, 275], which will be simply named surface plasmon resonance (SPR) within this thesis. A schematic representation of the SPP is displayed in Fig 2.15. As the dimension of the particle is much smaller than the wavelength of the incident light, the absorption of the electromagnetic wave leads to the excitation of a localized plasmon oscillation within the nanoparticle. The incoming photons drive the electrons of the conduction band collectively with respect to the positive lattice ions, which causes a net charge difference on 


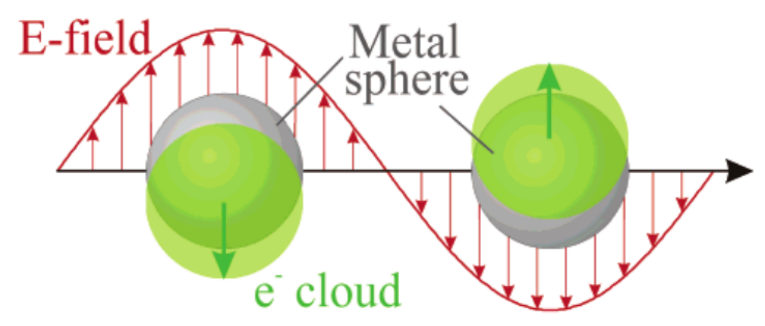

Figure 2.15.: Schematic of a plasmonic oscillation of a sphere, showing the displacement of the conduction electrons relative to the nuclei. The lattice ion exerts a restoring force on the electrons. The surface plasmon resonance depends on the strength of this restoring force, i.e. the optical properties of the material. From [276] with kind permission of the American Chemical Society.

the surface of the particle [274]. In other words, the absorption of the incident light induces a dipole oscillation in the metallic nanoparticle, emitting an electromagnetic wave. If the frequency of the radiating dipole is resonant with the incident light, even small incident fields lead to a significant amplitude of the induced electromagnetic field, which is only limited by radiative [277] and non-radiative [278] damping processes. The resonance frequency of the metallic particle depends mainly on the particle size [279 281], the polarizability of the metal [282] and the surrounding medium [276, 283]. In the past years, the electromagnetic mechanism was inten-

(a)

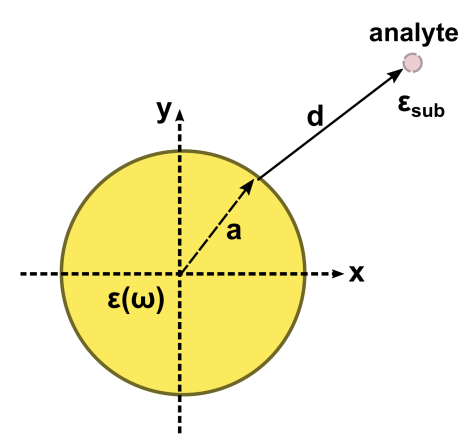

(b)

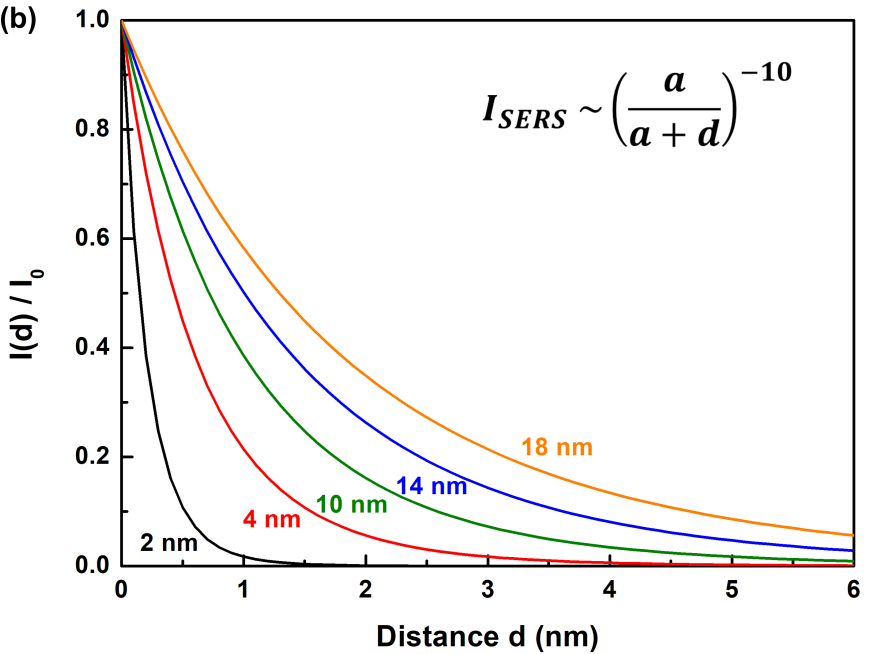

Figure 2.16.: (a) A schematic of a typical SERS experiment. In the vicinity of a metallic particle with radius $a$ and dielectric constant $\epsilon(\omega)$, a probed analyte resides in a dielectric environment with the dielectric constant $\epsilon_{\text {sub }}$. (b) Distance dependence of the SER intensity $I(d)$, normalized to the SER intensity directly on the particle surface $I_{0}$, for different particle radii $a$. 
sively reviewed [68, 284 287] and can be explained within classical electrodynamics [288 290]. A simplified schematic diagram of the electromagnetic mechanism in SERS is shown in Fig.2.16a. A nanometer-sized sphere with the complex dielectric constant $\epsilon(\omega)$ resides in a surrounding medium with a dielectric constant $\epsilon_{\text {sub }}$. The diameter of the sphere is small compared to the wavelength of the exciting light. A analyte in the vicinity of the sphere is exposed to an electric field $\vec{E}_{\mathrm{M}}$, which is a superposition of the incident field and the field of the induced dipole within the metallic sphere, i.e. the electric field of the excited surface plasmon. The field enhancement $A(\omega)$ experienced by the analyte is the ratio of the electric field $\vec{E}_{\mathrm{M}}$ at the position of the analyte and the incoming field:

$$
A(\omega)=\frac{\vec{E}_{\mathrm{M}}}{\vec{E}_{\mathrm{inc}}} \sim \frac{\epsilon(\omega)-\epsilon_{\text {sub }}}{\epsilon(\omega)+2 \epsilon_{\text {sub }}}\left(\frac{a}{a+d}\right)^{3}
$$

In resonance, i.e. $\operatorname{Re}(\epsilon(\omega))=-2 \epsilon_{\text {sub }}$, the enhancement is particularly strong. Additionally to the incoming field, the Raman scattered photons will also be affected by the field enhancement, if the Stokes or Anti-Stokes scattered field is in resonance with the surface plasmon of the metallic sphere. Therefore, taking into account both of the enhancing effects, the electromagnetic enhancement factor $G(\omega)$ for the Raman signal is given by

$$
G\left(\omega_{S}\right)=\left|A\left(\omega_{L}\right)\right|^{2}\left|A\left(\omega_{S}\right)\right|^{2} \sim\left|\frac{\epsilon\left(\omega_{L}\right)-\epsilon_{\text {sub }}}{\epsilon\left(\omega_{L}\right)+2 \epsilon_{\text {sub }}}\right|\left|\frac{\epsilon\left(\omega_{S}\right)-\epsilon_{\text {sub }}}{\epsilon\left(\omega_{S}\right)+2 \epsilon_{\text {sub }}}\right|\left(\frac{a}{a+d}\right)^{12}
$$

where $A\left(\omega_{L}\right)$ and $A\left(\omega_{L}\right)$ are the enhancement factors of the laser field and the Raman scattered field respectively. Although Eq. (2.19) is based on a simple model, it includes important properties of the electromagnetic enhancement mechanism. If the wavelength of the Raman scattered photons is in the vicinity of the excitation wavelength, one can approximate $A\left(\omega_{L}\right) \sim A\left(\omega_{S}\right)$, resulting in the well-known $|\vec{E}|^{4}$ approximation [68, 71, 246, 287].

Furthermore, Eq.(2.19) shows that the EM enhancement is a long range effect and does not require the direct presence of the analyte at the surface of the metallic particle. Within the $|\vec{E}|^{4}$ approximation, the rapid decay of the dipole field, $\vec{E} \sim 1 / d^{3}$ results in a SER intensity decrease by $1 / d^{12}$ as shown in Eq. 2.19) in Fig 2.16p. However, the presence of a molecular monolayer on the surface of the particle increases its surface area, resulting in a mitigation of the distance dependence [291]

$$
I_{\mathrm{SERS}} \sim(a /(a+d))^{10}
$$

Although the obtained relation between the SER intensity $I_{\text {SERS }}$ and the distance $d$ from the nanoparticle is, strictly speaking, only valid for spherical particles, similar observations were made for oblate particles as well 284. In this case, the spherical radius $a$ refers to the local curvature of the particle. Within the last years, the valid- 
ity of Eq.2.20 has been supported by several experimental studies [73, 74, 291, 292] and is today a generally accepted approximation for the distance dependence of the electromagnetic enhancement in SERS.

A surface plasmon can be excited in a single spherical nanoparticle [279 281, 293], aggregates of nanoparticles [280, 294] and in electrochemical roughened metallic surfaces [273, 295, 296]. Furthermore, different shapes of particles such as rods [297], disks [298], triangular prisms [299] and nanoshells [300] can be used, although for all of them different resonance conditions have to be fulfilled. The resonance frequency further depend on the optical properties of the metal and the particle radius $a$ of the nanostructure, as can be seen in Eq.2.19). Several numerical methods were used to analyze the size dependence of the surface plasmon's resonance frequency [279-281], revealing a red shift with increasing particle size (see Fig,2.17a). In addition, the

(a)

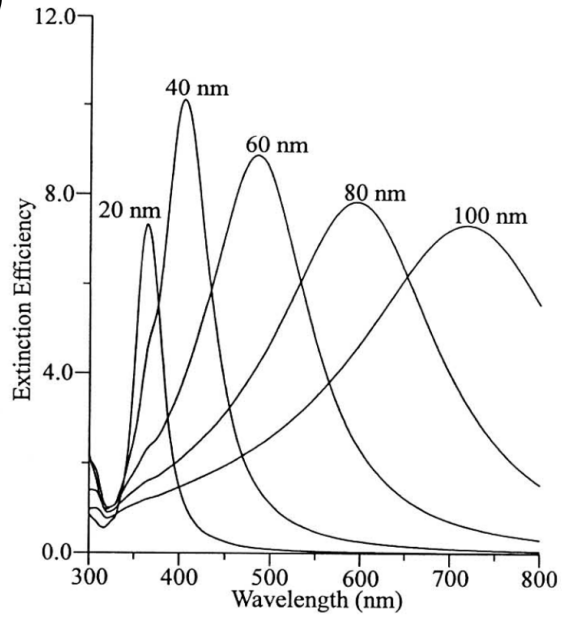

(b)

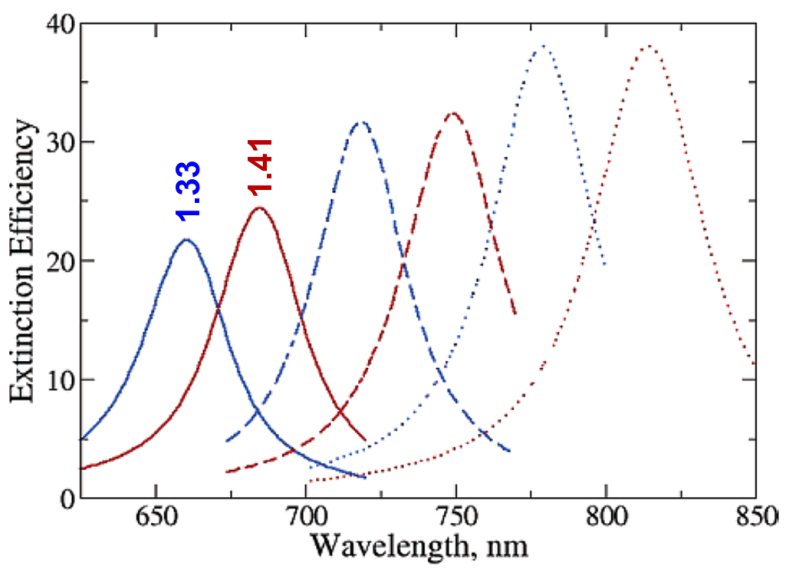

Figure 2.17.: (a) The peak wavelength of the surface plasmon, here exemplarily shown for Ag, depends on the particle size and experiences a red shift with increasing particle size. From [301 with kind permission of Springer. (b) Extinction efficiency of gold nanocylinders with different dimensions in two media $(n=1.33$ in blue and $n=1.41$ in red): $40 \mathrm{~nm} \times 20 \mathrm{~nm}$ cylinders (solid), $50 \mathrm{~nm} \times 20 \mathrm{~nm}$ cylinders (dashed) and $60 \mathrm{~nm} \times 20 \mathrm{~nm}$ cylinders (dotted)). A refractive index $n>1$ result in a red shift of the resonance wavelength. Taken from [283] with kind permission of the American Chemical Society

peak wavelength $\lambda_{\mathrm{SPR}}$, i.e. the resonance frequency, depends on the dielectric function as well as the interparticle distance of two particles. Embedding the particle in a dielectric environment with $n>1$ and $\epsilon_{\text {sub }}>1$, respectively, the peak wavelength of the surface plasmon will shift to higher wavelength compared to the free particle, residing in air or vacuum (see Fig 2.17b). Numerical calculations, e.g. within the discrete dipole approximation (DDA), showed a linear dependence of $\lambda_{\mathrm{SPR}}$ with respect to the change of the refractive index of the surrounding medium [276, 283]. 
Such behavior is also independent of the shape and size of the particles in a colloid solution [283]. However, while colloid nanoparticles reside in a homogeneous surrounding medium (the solvent), particles deposited on a planar surface are exposed to different dielectric conditions simultaneously. This fact was first addressed by YAMAGUCHI et al. [302, who expressed such a mixed environment by means of an effective dielectric constant $\epsilon_{\mathrm{eff}}$ (alternatively, the refractive index can be used, since $n \approx \sqrt{\epsilon})$. It represents the mean of the dielectric constant of the substrate $\epsilon_{\text {sub }}$ and of the remaining ambient medium $\epsilon_{\mathrm{M}}$ [303]. Thus, the effective dielectric constant is determined by

$$
\epsilon_{\mathrm{eff}}=m \cdot \epsilon_{\mathrm{sub}}+(1-m) \cdot \epsilon_{\mathrm{M}}
$$

The mixing factor $m$ describes the ratio of the particle fraction in contact with the substrate. In the visible region, the dielectric constant of the substrate is assumed to be independent of the frequency [303]. Furthermore, the model by YAMAGUCHI et al. included particle-particle interactions, which lead to an additional red shift of the resonance frequency. This is further supported by numerical calculations of the plasmon frequency and the electric field enhancement of single particles and dimers [293]. A huge increase of the electric field enhancement and a red shift of the resonance frequency by decreasing the interparticle distance were observed, which is in contrast negligible small for distances exceeding a tenth of the particles size [293]. Although the discussed influences on the resonance frequency are mostly analyzed for spherical particles within Mie theory [304, 305] and DDA, they constitute a universally valid concept for the surface plasmon frequency of metallic particles of arbitrary shape [276, 279 281, 283, 306]. Additionally to the red shift of the surface plasmon, the small distance between two particles can result in a so-called hot spot. These hot spots are prerequisite to observe single-molecule SERS [307] and distinguishes oneself by an extremely large enhancement at the junction between both particles [308 310]. Because of the required small distance between two particles, the hot spot is highly localized [311]. Within such spot an enhancement by a factor of $10^{11}$ can be accomplished [246].

To conclude this brief discussion of the electromagnetic enhancement, possible damping mechanisms will be addressed. Since the optical properties of the metal, i.e. the dielectric function, play an important role in the EM mechanism, one has to keep in mind that $\epsilon(\omega)$ is a complex quantity. While the real part has to fulfill certain criteria with respect to the resonance conditions, the imaginary part represents a damping of the plasmon resonance and, therefore, has to be as small as possible. To characterize the damping, a quality factor $Q$ is introduced [246]

$$
Q=\frac{\omega\left(\mathrm{d} \epsilon^{\prime} / \mathrm{d} \omega\right)}{2 \epsilon^{\prime \prime 2}}
$$

where $\epsilon^{\prime}$ is the real party and $\epsilon^{\prime \prime}$ is the imaginary part of the dielectric function $\epsilon(\omega)$. 
This quality factor leads to the following two criteria:

- $\operatorname{Re}(\epsilon)$ should be negative (rule of thumb: $-20 \leq \operatorname{Re}(\epsilon) \leq-1$ )

- $\operatorname{Im}(\epsilon) \ll 1$ and $Q>2$
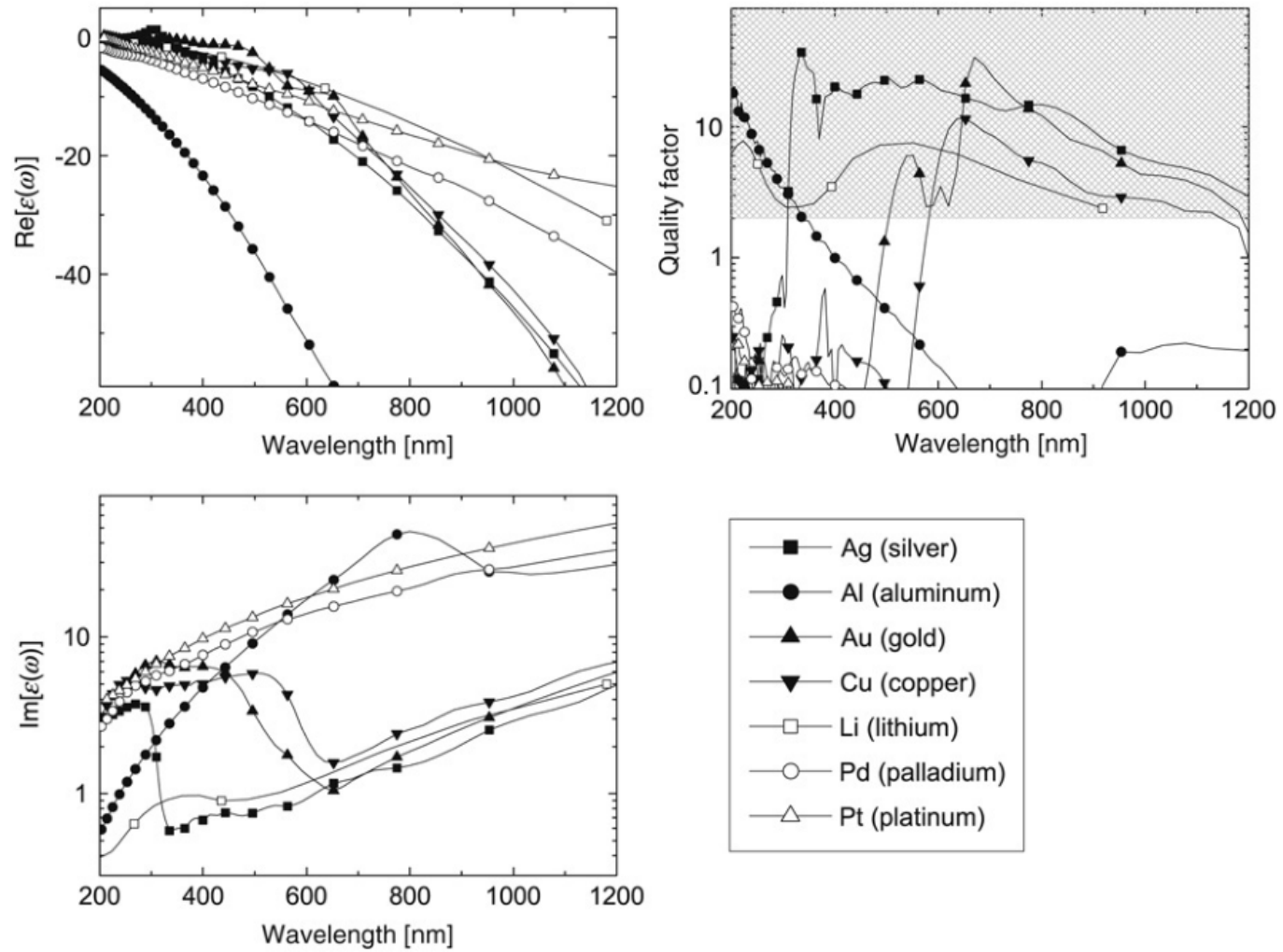

Figure 2.18.: Overview of the optical properties of a few selected metals. Real (top) and imaginary (bottom) parts of $\epsilon(\omega)$ are plotted as a function of the wavelength in the left column. Top right, the wavelength-dependence of the quality factor $Q$, defined in Eq.(2.22), of the localized surface plasmon resonance for metal/air particle is shown. The shaded area marks the region, which is interesting for most plasmonic applications. Taken from Ref.[246] with the kind permission of Elsevier Science.

These two criteria provide a reasonable basis for the selection of a plasmon-active metal for the spectral region of interest. While aluminum fulfills perfectly the requirements for the excitation of surface plasmons in the UV region, silver and gold are the metal of choice for the visible region. The wavelength dependent behavior of the real and imaginary part of $\epsilon(\omega)$ is summarized in Fig 2.18. In the case of gold, interband transitions, where bound electrons are optically excited to higher energy bands, alter the optical properties in the visible region [312]. This leads to an increase of the imaginary part of the dielectric function, which results in the damping of the plasmon band. Such interband excitation of a electron-hole pair is also 
known as Landau damping [313. In the case of silver, these transitions occur in the UV region [314] making their contributions negligible for typical SERS experiments. The difference in the optical properties of both metals is the basic reason for the higher SERS-activity of silver compared to gold [246].

Small particles show a damped and broadened plasmonic excitation, which may disappear in particles smaller than $2 \mathrm{~nm}$ in diameter [315]. This behavior is related to the mean free path of the electrons in the metal. Since the particle is smaller than the bulk value of the mean free path, $d=35-50 \mathrm{~nm}$ [316, additional scattering of the electron at the particle surface dampens the surface plasmon [278]. However, in particles with a dimension similar to the excitation wavelength, the dipole approximation is no longer valid and higher order modes are excited, which are blue shifted with respect to the dipole band. Furthermore, radiation effects [277] come in play and scattering begins to dominate over absorption [246, 274], both resulting in a further damping and broadening of the plasmonic response. Note that, these damping mechanisms should not be confused with the broadening of the surface plasmon due to the presence of a polydisperse particle size. However, they often cannot be distinguished from one another in absorption and extinction measurements [246].

\subsubsection{Chemical (electronic) enhancement}

Additional to the electromagnetic enhancement caused by the surface plasmon resonance, chemical and electronic effects may play a role as well and it might even be crucial for single-molecule detection [317, 318]. Chemical contributions to the enhancement are short-range effects and require a direct contact between the analyte and the metallic nanostructure [317, 318. However, it has been proven that the selective study of the chemical contributions is quite challenging. This is mostly due to the small contribution to the enhancement by a factor of only $10-10^{2}$, in contrast to the huge electromagnetic contribution of $10^{4}-10^{8}$.

First evidence for chemical contributions was provided by electrochemical measurements [319]. When a molecule lies flat on the surface, the enhancement factor depends on the applied electrode potential [319, 320]. This fact could not be solely explained by an electromagnetic enhancement due to surface plasmons. Since then, several studies dealt with the chemical enhancement, its contributions and the underlying mechanisms [233, 318, 321 324]. As shown in Fig,2.19, the chemical enhancement can generally be explained by a resonant-like Raman mechanism [318, 321 323]. In a simplified picture, a charge transfer from the metal to the adsorbate, or vice versa, induces an additional intermediate electronic state. This state is in resonance with the excitation wavelength, thus, enhancing the Raman signal by a resonant-like Raman process. For such a charge transfer, however, a chemical bond has to be created between the metal and the analyte, which restricts chemical contributions to the first monolayer [318]. The similarity to a resonant Raman process allows the description of the chemical enhancement based on Frank-Condon 


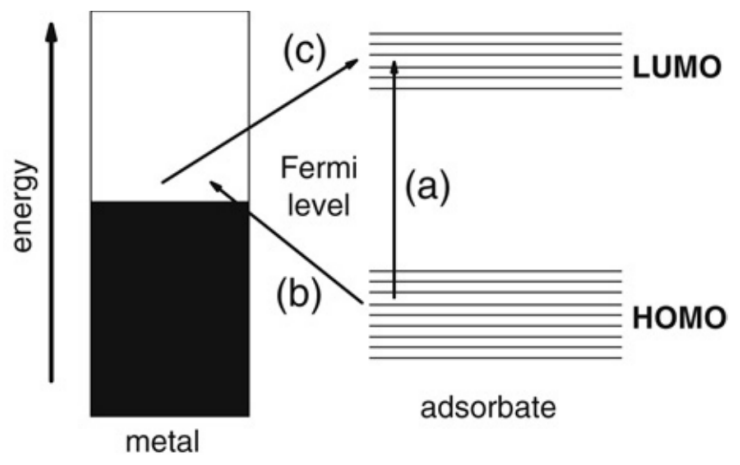

Figure 2.19.: The chemical enhancement can be viewed as charge transfer mechanism, appearing as a resonant-like contribution to the measured intensity. An electronic transition of the metal-molecule complex can be directly excited by the incident laser energy (a). Indirect coupling via charge transfer, by means of the Fermi level of the metal can induce a intensity enhancement. This charge transfer can occur from the HOMO of the analyte to the Fermi level of the metal (b) or from the Fermi level to the molecule's LUMO (c). However, such charge transfer is limited to the first atomic layer, which is chemically bonded to the metal. From Ref.[246] with the kind permission of Elsevier Science.

and Herzberg-Teller coupling mechanisms [321, 322]. A static contribution to the enhancement can further be provided by the chemical coupling between the metal and the analyte. This coupling may alter the electronic structure of the probed system, changing the polarizability of a given mode and, thus, vary the observed Raman intensity. Both effects, the charge-transfer and static contribution, are often summarized as the mechanisms behind the chemical enhancement. Sometimes, the resonant excitation of electronic states of the free molecule are included in the description of the chemical enhancement additionally, although they are also present in the absence of the metallic nanostructure [324]. Because of its chemical specificity, this mechanism depends on the adsorption site, the geometry of the bonding and the energy levels of the probed system [325, 326]. Once again, it should be emphasized that chemical contributions to the enhancement are only provided if a chemical interaction by bond formation is present in the metal-molecule complex. Although the chemical enhancement can be qualitatively explained, its magnitude and even, whether it is in fact present, is still highly under debate. The common opinion, however, is that the corresponding enhancement factor ranges from 10 to $10^{2}$ and that its contribution to the overall enhancement is significantly smaller than that of the electromagnetic enhancement. 


\subsubsection{Enhancement factors}

To characterize the possible enhancement of a given SERS substrate, one calculates the so-called SERS enhancement factor $E F$. In a first approximation, the overall enhancement factor can be expressed by the ratio of the SER intensity and the conventional Raman intensity, which is also referred to as intensity contrast $C=I_{\mathrm{SERS}} / I_{\mathrm{RS}}$. This contrast, however, ignores the fact that the intensity of a Raman scattering experiment depends on the amount of molecules within the scattering volume. Therefore, the intensity is typically normalized to the number of molecules $N_{\mathrm{V}}$ in the scattering volume $V$ of the conventional Raman experiment and to the average number of adsorbed molecules $N_{\mathrm{S}}$ in the same volume in the SERS experiments [78]. This results in the following SERS enhancement factor EF

$$
E F=\frac{I_{\mathrm{SERS}} / N_{\mathrm{S}}}{I_{\mathrm{RS}} / N_{\mathrm{V}}}
$$

Such a simple definition of the $E F$ is rather intuitive and is not explicitly derived from the Raman cross section. Based on the enhancement experienced by a single molecule, LE RU and co-workers [78] introduced a more rigorous definition of the $E F$ and provided, this way, a physical background for the intuitive definition of the $E F$ as shown in Eq.2.23). By deriving the enhancement of the cross section of a single molecule and linking it to the Raman cross section in the conventional Raman experiment, they obtained the following expression for the $E F$

$$
E F=\frac{I_{\mathrm{SERS}} H_{\mathrm{eff}} c_{\mathrm{RS}}}{I_{\mathrm{RS}} \mu_{\mathrm{S}} \mu_{\mathrm{M}} A_{\mathrm{M}}}
$$

where $\mu_{\mathrm{S}}$ is the surface density of the analyte on a single metal nanoparticle, $\mu_{\mathrm{M}}$ the surface density of the individual particles, $A_{\mathrm{M}}$ the surface area of the particle, $H_{\text {eff }}$ the effective height of the scattering volume and $c_{\mathrm{RS}}$ the concentration of the analyte in the conventional Raman scattering experiment. Comparing this rigorous definition of the $E F$ to the intuitive one of Eq.(2.23), $N_{\mathrm{S}}$ would be represented by $N_{\mathrm{S}}=\mu_{\mathrm{S}} \mu_{\mathrm{M}} A_{\mathrm{M}}$ and the number of molecule in the conventional experiment by $N_{\mathrm{V}}=H_{\mathrm{eff}} c_{\mathrm{RS}}$. 


\subsection{Polaronic excitations probed by Raman spectroscopy: the status quo}

As the study of the polaronic behavior is the main interest of this thesis, a brief summary of the current knowledge of polaronic characteristics in the Raman spectra of manganites and their correlations with other experimental data will be given in the following.

Over the last decades, numerous Raman spectroscopy studies of mixed-valence manganites were published. Most of them discussed the relations between the temperature-dependent Raman spectra and the polaronic behavior. A characteristic property of the Raman spectra of orthorhombic manganites are the two high frequency modes, namely the anti-symmetric stretching vibration at $490 \mathrm{~cm}^{-1}$ and the symmetric stretching vibration at $611 \mathrm{~cm}^{-1}$. Both modes are linked to an occurring Jahn-Teller distortion in the system [58, 59, 262], which is further supported by resonant Raman experiments on $\mathrm{LaMnO}_{3}$ [327, 328]. By exciting the sample near the Jahn-Teller energy $\left(E_{\mathrm{JT}} \approx 2 \mathrm{eV}\right.$ in $\left.\mathrm{LaMnO}_{3}[327]\right)$, a strong enhancement of the stretching modes is observed indicating a resonant behavior. High-temperature studies further showed the expected melting of the cooperative Jahn-Teller distortion at $T \approx 800 \mathrm{~K}$ [329]. A comprehensive Raman study focusing on structural relations between the observed Raman frequencies and the tilting angle of the $\mathrm{MnO}_{6}$ octahedra was made by MARTIN-CARRÓN [262, who established a relation between the intensity and width of the broad Jahn-Teller bands and the dynamic fluctuations produced by polaron hopping.

Temperature-dependent studies showed a strong correlation between the JT modes and the metal-insulator transition [61, 65-67, 267]. With decreasing temperature, the intensity of the stretching vibrations as well as the resistivity increases, reaching a maximal value at $T_{\mathrm{MI}}$, while both decrease rapidly when passing through the MI transition [61]. A similar behavior was observed by LiAROKAPIS et al., who measured the temperature-dependent Raman spectra of $\mathrm{La}_{1-x} \mathrm{Ca}_{x} \mathrm{MnO}_{3}$ with different dopings [267]. While they observed a decrease of the JT modes for dopings of $x=0.3$ and $x=0.4$, the low-doped compound with $x=0.05$ shows a similar behavior as the parent compound $\mathrm{LaMnO}_{3}$ [329], meaning that the stretching modes gain in intensity with decreasing temperature. Pressure-dependent Raman spectra show a similar behavior in comparison with the temperature-dependent measurements in the low pressure regime, $p<7 \mathrm{GPa}$, which is ascribed to a symmetrization of the $\mathrm{MnO}_{6}$ octahedra and, consequently, to a reduction of JT distortions [67]. At higher pressures, however, the metallization is suppressed due to the strong lattice compression, which activates charge localizing antiferromagnetic interactions [67]. Moreover, the linewidth has a pronounced minimum at $T_{\mathrm{C}}$ 61] indicating a long polaron lifetime $\tau_{\mathrm{JT}}$. ABRASHEV et al. estimated the maximum polaronic lifetime at $T_{\mathrm{C}}$ to be $\tau_{\mathrm{pol}}=6.7 \mathrm{ps}$, which is in good agreement with the one estimated from 
the resistivity data [61]. In addition, the stretching modes show a distinct softening below $T_{\mathrm{C}} / T_{\mathrm{N}}$, which can be attributed to spin-phonon coupling [62, 63, 267, 330].

A first attempt to explain the observed temperature-dependent behavior was made by ILIEv et al. [65], who also took the mixed-valence state and the observed broadening of the Jahn-Teller modes into account. The doping of the earth alkaline metal induces a mixed-valence state of the $\mathrm{Mn}$ ion resulting in a disorder of the $\mathrm{Mn}^{3+}$ and $\mathrm{Mn}^{4+}$ ions. In such a disordered state, the coherent Jahn-Teller distortion presented in the parent compound $\mathrm{LaMnO}_{3}$ is disturbed. A non-coherent JT distortion develops and, as a consequence, a disorder within the $\mathrm{MnO}_{6}$ octahedral network and the oxygen arrangement, respectively, is created causing a broadening of the JahnTeller stretching vibrations [65]. In this case, the JT stretching vibrations do not correspond to a first order $\Gamma$-point phonon anymore but, indeed, they belong to the phonon density of states of the oxygen atoms [65. Temperature-dependent measurements [65] further showed a decrease in intensity of the JT modes when passing through the metal-insulator transition as well as the increase of a sharp mode around $440 \mathrm{~cm}^{-1}$, attributed to a bending mode of the $\mathrm{MnO}_{6}$ octahedron. Because of its narrow linewidth and the associated sharpness, the emerging mode was attributed to a $\Gamma$-point phonon indicating the development of a more ordered structure at low temperatures [65. Such a behavior can be explained by the mean lifetime of the Jahn-Teller distortion $\tau_{\mathrm{JT}}$ and the time between two consecutive $\mathrm{Mn}^{3+} \rightarrow \mathrm{Mn}^{4+}$ hops $\tau_{\mathrm{h}}$, i.e. the averaged $\mathrm{Mn}^{3+}$ lifetime [65]. In the nonmetallic paramagnetic (as well as in the antiferromagnetic and ferromagnetic phases at doping levels below $x=0.5)$, the lifetime of $\mathrm{Mn}^{3+}$ is much larger than the period in which the JahnTeller distortion develops. Therefore, a noncoherent static Jahn-Teller distortion within the manganite system is formed. In the metallic state, however, the hopping time $\tau_{\mathrm{h}}$ strongly decreases to a point where $\tau_{\mathrm{h}} \ll \tau_{\text {pol }}$ and, thus, the Jahn-Teller distortion can only develop partly within the time frame of a $\mathrm{Mn}^{3+}$ ion. Hence, the Jahn-Teller distortion is reduced, i.e. the intensity of the stretching vibrations in the Raman spectra decreases, and a more ordered structure develops giving rise to the sharp bending mode. However, the JT distortion as well as the oxygen disorder, does not vanish completely, as remnants of the JT modes are still visible in the Raman spectra for temperatures $T<T_{\mathrm{MI}}$. This supports the framework of electronic phase separation in the mixed-valence manganites [65, 66]. Additionally to $\mathrm{La}_{0.7} \mathrm{Ca}_{0.3} \mathrm{MnO}_{3}$ a similar disorder-order transition was detected in the chargeordered manganite $\mathrm{Pr}_{0.65} \mathrm{Ca}_{0.35} \mathrm{MnO}_{3}$ [331 and the parent compound $\mathrm{LaMnO}_{3} 64$ and seems to be quite common phenomena in manganite systems. While the transition in $\mathrm{Pr}_{0.65} \mathrm{Ca}_{0.35} \mathrm{MnO}_{3}$ describes a transformation from a dynamical JT distortion to a static one below the charge-ordering temperature $T_{\mathrm{CO}}=225 \mathrm{~K}$, the transition in $\mathrm{LaMnO}_{3}$ is based on the thermally-activated disorder of the e $\mathrm{g}$ orbitals of $\mathrm{Mn}^{3+}$ for $T>T_{\mathrm{JT}} \approx 705 \mathrm{~K}$. At this point, this thesis ties in with the current state of research and presents, amongst others, temperature-dependent Raman measurements of the prototypical CMR system $\left(\mathrm{La}_{0.65} \mathrm{Pr}_{0.45}\right)_{0.7} \mathrm{Ca}_{0.3} \mathrm{MnO}_{3}$ (LPCMO), presented in the following $\mathrm{Ch} 3$. 


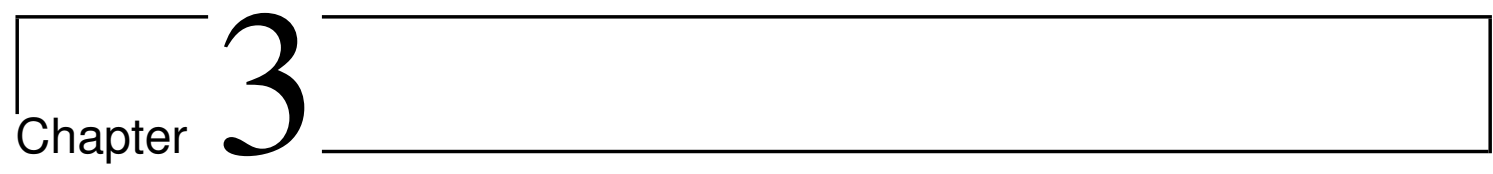

\title{
Publication 1
}

\section{Intrinsic antiferromagnetic coupling underlies colossal mag- netoresistance effect - Role of correlated polarons}

\author{
V. Moshnyaga ${ }^{1}$, A. Belenchuk ${ }^{2}$, S. Hühn ${ }^{1}$, C. Kalkert ${ }^{1}$, M. Jungbauer ${ }^{1}$, O.I. Lebedev ${ }^{3}$, S. \\ Merten $^{1}$, K.-Y. Choi ${ }^{4,5}$, P. Lemmens ${ }^{4}$, B. Damaschke ${ }^{1}$ and K. Samwer ${ }^{1}$ \\ ${ }^{1}$ I. Physikalisches Institut, Georg-August University Göttingen, Friedrich-Hund-Platz 1, \\ D-37077, Göttingen \\ 2 IIEN, Academy of Sciences of Republic Moldova, Strada Academiei 3/3, MD-2028, \\ Chisinau, Republic of Moldova. \\ ${ }^{3}$ Laboratoire CRISTMAT, UMR 6508 CNRS-ENSICAEN, 6 Boulevard du Maréchal Juin, \\ 14050 CAEN Cedex, France \\ ${ }^{4}$ Institut für Physik der Kondensierten Materie, Technische Universität Braunschweig, \\ Mendelssohnstrasse 3, D-38106, Braunschweig \\ ${ }^{5}$ Department of Physics, Chung-Ang University, Seoul 156-756, Republic of Korea
}

Physical Review B 89, 024420 (2014)

DOI: 10.1103/PhysRevB.89.024420

Accepted for publication on November $22^{\text {th }}, 2013$

Published online January $30^{\text {th }}, 2014$

Reproduced from Physical Review B 89, 024420 (2014) with the permission of APS Publishing.

Remark: Figures were partly rearranged to fit into the format of this thesis. Furthermore, figure 1 and 2 of the original manuscript were merged into one (Figure 3.1 in this thesis). 


\subsection{Motivation and Summary}

A commonly believed picture of the colossal magnetoresistance (CMR) effect is related to a first-order phase transition and electronic phase separation with coexisting ferromagnetic metallic and antiferromagnetic insulating phases. However, the underlying mechanism, i.e. the characteristic energy scale of the interacting phases and their spatial extent, still remains under debate. In the following study, experimental evidence of the existence of an effective antiferromagnetic coupling between the ferromagnetic nanodomains in epitaxial thin films of a classical CMR material $\left(\mathrm{La}_{1-y} \mathrm{Pr}_{y}\right)_{0.67} \mathrm{Ca}_{0.33} \mathrm{MnO}_{3}$ with a $\mathrm{Pr}$ dopings of $y=0.375$ and 0.4 is presented. This coupling leads to a peculiar low-field CMR behavior with magnetic hysteresis and slow resistance relaxation, both induced by the magnetization reversal. The coercive field obeys a square-root temperature dependence for $T \ll T_{\mathrm{C}}$ and increases anomalously close to the phase transition. The magnetic structure within the phaseseparation scenario could be modeled as an assembly of single-domain ferromagnetic nanoparticles, antiferromagnetically coupled (pinned) by correlated Jahn-Teller polarons. The concentration of polarons increases drastically close to phase transition as indicated by the third harmonic of the electrical conductivity as well as by Raman spectroscopy.

\subsection{Introduction}

The colossal magnetoresistance (CMR) effect, manifested itself as a drastic decrease of electrical resistance in an applied magnetic field, was first observed in single crystals of perovskite manganites in 1970 332. The "rediscovering" of CMR in thin manganite films [21, 22] initiated an enormous boom of research focusing on the intriguing CMR physics as well as on the potential applications. After almost two decades of extensive experimental and theoretical studies a commonly believed picture has been established: the fundamentals of CMR are related to a first order phase transition and electronic phase separation with coexisting ferromagnetic (FM) metallic and antiferromagnetic (AFM) insulating phases. The generic phase diagram of CMR manganites [164] with FM and AFM phases underscores a competition between two driving tendencies: electron delocalization, favoring a FM phase, and localization, which stabilizes an AFM ground state. These competing interactions result in the coexistence of FM metallic and charge-ordered insulating (COI) phases [180] close to a first order magnetic transition if the strength of electron-phonon coupling is large enough. "Colossal" values of CMR, $\rho(H) / \rho(0) \approx 10^{6} \%$ were indeed observed close to the FM/AFM phase boundary, achieved both by "filling control" close to $x \approx 0.5$ doping in the $\mathrm{La}_{1-x} \mathrm{Ca}_{x} \mathrm{MnO}_{3}$ system [333] and by "bandwidth control" while substituting a large La-cation by a smaller $\mathrm{Pr}$ in $\mathrm{La}_{5 / 8-y} \mathrm{Pr}_{y} \mathrm{Ca}_{3 / 8} \mathrm{MnO}_{3}$ (LPCMO) [152. Electronic phase coexistence was believed to govern the magnetotransport in manganites close to phase transition at $T_{\mathrm{C}}$, although the energy scale and the nature of magnetic interactions between the competing phases still remain 
unclear. SEN et al. [159] have demonstrated theoretically a coexistence of FM and nm-size AFM phases even in the absence of A-site disorder [334] but at sufficiently large electron-phonon coupling. Correlated Jahn-Teller (JT) polarons [146] or bipolarons [171] (CP), detected in neutron and x-ray scattering [144, 145, 149, 335] 337. as a short-range-ordered lattice superstructure with a correlation length of $\delta \approx 1-2 \mathrm{~nm}$ and charge/orbital ordering (COO) of CE-type, may contribute to the nm-scale phase separation. Considering the ground state of the CE-phase to be AFM, one can suggest that CPs may also possess short-range AFM correlations. However, the estimated very small amount of CPs [149, 336, 337], questions their role in the complex magnetic and electric state close to $T_{\mathrm{C}}$. Here we report that the $\mathrm{CMR}$ in high quality and strain-free epitaxial $\left(\mathrm{La}_{0.6} \mathrm{Pr}_{0.4}\right)_{0.67} \mathrm{Ca}_{0.33} \mathrm{MnO}_{3} / \mathrm{MgO}(100)$ films is characterized by a low-field hysteresis and slow relaxation dynamics, both originating from a peculiar magnetic domain structure with nm-size FM domains, antiferromagnetically coupled by correlated Jahn-Teller (JT) polarons. Our experimental results confirm the earlier theoretical models of electronic phase separation with competing FM metallic and AFM insulating phases [159, 338] at the first order phase transition. Moreover, we demonstrate that even a tiny amount of the polaronic AFM phase stabilizes the FM nano-domains and induce an effective exchange coupling between them, thus, playing a decisive role in the spin polarized charge transport and in the CMR behavior in general.

\subsection{Sample preparation and experimental techniques}

LPCMO films were prepared by a solution-based and vacuum-free metalorganic aerosol deposition (MAD) technique [98]. The precursors, acetylacetonates of La, $\mathrm{Pr}, \mathrm{Ca}$ and $\mathrm{Mn}$, have been weighted in appropriate amounts to obtain the experimentally determined molar ratios in the solution, i.e. La:Pr:Ca:Mn $=0.57: 0.34: 0.40: 1$. Then they have been dissolved in dimethylformamide to a concentration $0.02 \mathrm{M}$ calculated on Mn-precursor and sprayed onto the heated $\mathrm{MgO}(100)$ substrate by using compressed air at a pressure, $p=5 \mathrm{~atm}$. Keeping the following typical MAD processing conditions, like substrate temperature, $T_{\text {sub }}=800-900^{\circ} \mathrm{C}$, the solution volume, $V=2 \mathrm{ml}$, and deposition time, $t=5 \mathrm{~min}$, one gets an LPCMO film with the thicknesses, $d \approx 60 \mathrm{~nm}$; the corresponding growth rate is $v \approx 12 \mathrm{~nm} / \mathrm{min}$. After deposition the films were cooled down to room temperature in $30 \mathrm{~min}$. For comparison $\mathrm{La}_{0.7} \mathrm{Ca}_{0.3} \mathrm{MnO}_{3}$ and $\mathrm{La}_{0.7} \mathrm{Sr}_{0.3} \mathrm{MnO}_{3}$ were grown on $\mathrm{MgO}(100)$ as described in details in Ref.[149]. The structure of the films was characterized at room temperature by X-ray diffraction (XRD, $\Theta-2 \Theta$ Bragg-Brentano geometry, $\mathrm{Cu}-\mathrm{K}_{\alpha}$ radiation), small-angle X-ray reflection (XRR), and transmission electron microscopy (TEM) as well as by scanning tunneling microscopy (STM). TEM and high resolution TEM (HRTEM) studies were carried out on cross-section sample using Tecnai G2 30 UT microscope operated at $300 \mathrm{kV}$ and having $0.17 \mathrm{~nm}$ point resolution. Cross-section sample for TEM measurements was prepared using a focus ion beam (FIB) machine (FEI Helios 600 NanoLab DualBeam instrument in CIC Nano GUNE). The 
four-probe dc and ac resistivity measurements were performed by using a PPMS from "Quantum Design" in the temperature range, $T=2-400 \mathrm{~K}$, and for magnetic fields, $\mu_{0} H=0-4 \mathrm{~T}$, applied parallel to the film plane. The amplitude and the frequency of the ac current were varied in the range, $J=0-400 \mu \mathrm{A}$, and $f=0$ $1000 \mathrm{~Hz}$, respectively. Along with the ac voltage at the fundamental frequency, $U_{\omega}$, a third harmonic signal, $U_{3 \omega}$, was evaluated by means of Fourier analysis of the measured ac signal. Magnetic measurements were carried out by using a commercial SQUID magnetometer MPMS from "Quantum Design" for $T=10-400 \mathrm{~K}$ and fields, $\mu_{0} H=0-4 \mathrm{~T}$, aligned parallel to the film plane. In addition the magnetooptical Kerr effect and Kerr ellipticity were measured by using He-Ne laser with polarization modulation at $50 \mathrm{kHz}$ in a close circle He-cryostat, $T=20-300 \mathrm{~K}$, and magnetic fields, $\mu_{0} H=0-1.5 \mathrm{~T}$, oriented at $45^{\circ}$ with respect to the film plane. The same experimental setup was used to measure the resistance relaxation for magnetic fields applied parallel to the film plane. Raman spectroscopy was studied by using a Raman confocal microscope (LabRam HR800-UV) with $\mathrm{Ar}^{+}$-laser excitation at the wavelength, $\lambda=488 \mathrm{~nm}$. The spectra were measured for $T=80-300 \mathrm{~K}$ by using a cryogenic table "Linkam THMS 600" continuously cooled by liquid $\mathrm{N}_{2}$. The sample temperature was controlled by pumping rate of $\mathrm{N}_{2}$ and by heating of the sample holder. The measured Raman spectra were corrected by subtracting the base line and by normalizing by the Bose-Einstein factor.

\subsection{Results}

(a)

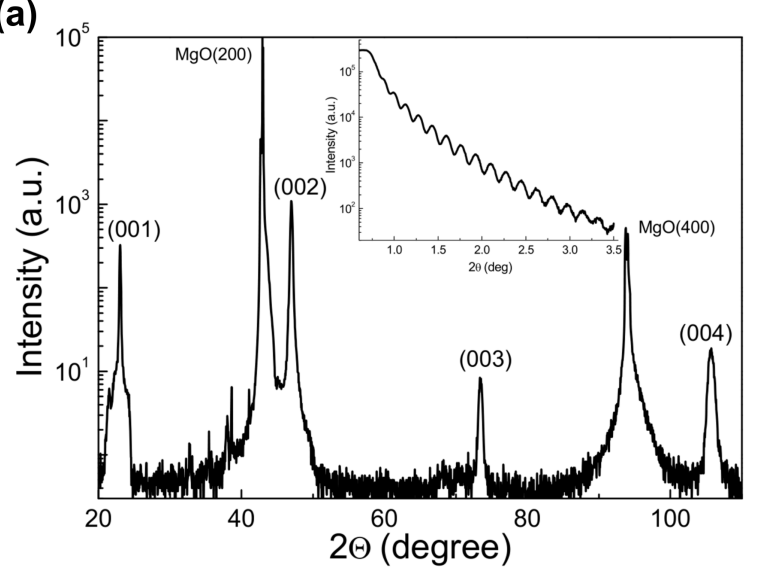

(b)

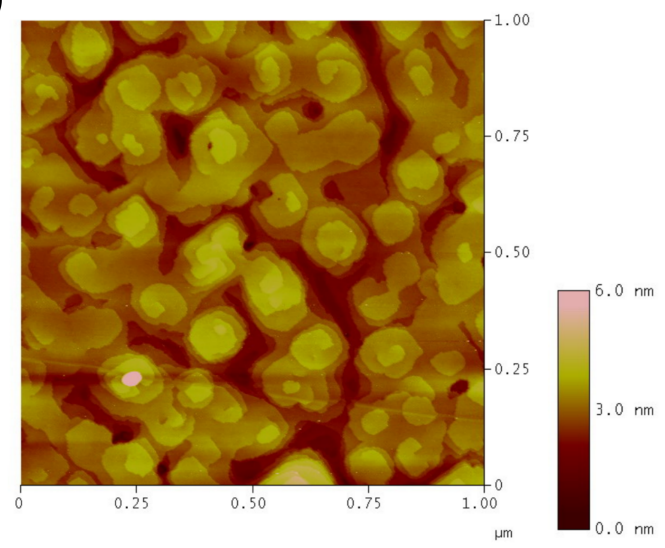

Figure 3.1.: (a) The $\Theta-2 \Theta$ pattern of an LPCMO/MgO film with $y=0.4$ shows the substrate peaks and (00l) peaks $(1=1,2,3,4)$, from the film of a perovskite structure, indicating an out-of-plane epitaxy with c-axis lattice parameter, $c=0.3870 \mathrm{~nm}$. From the periodicity of XRR oscillations (see the inset) the thickness, $d=53.6 \mathrm{~nm}$, was obtained. (b) STM image of the studied $\mathrm{LPCMO} / \mathrm{MgO}$ film $(y=0.4)$ with a mean square roughness, $\mathrm{RMS}=0.6 \mathrm{~nm}$. 
XRR measurements (see the inset to Fig 3.1 a) indicate a large scale homogeneity of the LPCMO films with the thickness, $d=50-70 \mathrm{~nm}$. XRD analysis (see Fig. $3.1 \mathrm{a}$ ) reveals a perfect out-of-plane epitaxy and a strain-free state of the films with a pseudo-cubic lattice parameter, $c \approx 0.3870 \mathrm{~nm}$, very close to the bulk value [152]. The STM image in Fig $3.1 \mathrm{~b}$ shows a surface morphology characteristic for epitaxial growth with atomically smooth terraces of 1 u.c. height and mean square roughness, RMS $=0.6 \mathrm{~nm}$, at the $1 \mu \mathrm{m}^{2}$ area. Moreover, an in-plane epitaxy of the film is evident with mosaic blocks oriented along two mutually perpendicular directions due to cubic crystalline structure of $\mathrm{MgO}$ substrate. TEM structural analysis shown in Fig. 3.2 infers a single crystalline character of LPCMO/MgO films and confirms STM morphology data concerning a large scale homogeneity and flatness of the films. The sample in Fig. $3.2 \mathrm{a}$ ) shows a uniform thickness of $\approx 50 \mathrm{~nm}$ in agreement with the nominal one and is characterized by the presence of twin boundaries, aligned perpendicular to the substrate surface. Electron diffraction (ED) analysis elucidates an orthorhombic Pnma structure with following lattice parameters: $a=0.544 \mathrm{~nm}$, $b=0.769 \mathrm{~nm}, c=0.545 \mathrm{~nm}$ at room temperature (\#ICSD 96908). The ED pattern in the insert of Fig. 3.2 a), being a superposition of those form $\mathrm{MgO}$ (substrate) and LPCMO (film), clearly evidences two main features of the film: 1) heteroepitaxial growth of the LPCMO film on the MgO substrate with the pseudo-cubic perovskite block $a_{\mathrm{p} \text {, LPCMO }}$ of the film aligned parallel to that of the substrate: $a_{\mathrm{p}, \mathrm{LPCMO}} / / a_{\mathrm{MgO}}$, and 2) twinning structure of LPCMO film. The HRTEM image of the LPCMO film (Fig. 3.2p) confirms the presence of rotational twins and reveals a heteroepitaxial and homogeneous interface. It should be noticed that due to very small orthorhombic distortion of LPCMO structure from a cubic perovskite, the lattice parameters of LPCMO, i.e. $a / \sqrt{2}, b / 2$, and $c / \sqrt{2}$, are very close to each other and can be expressed as $a_{\mathrm{p}, \text { LPCMO}}$. In this respect, the geometric phase analysis (GPA) patterns points out a uniform strain in both normal to the growth direction (g100) and parallel to the LPCMO/MgO interface (g010) directions. No twin boundary can be distinguishing in GPA color strain analysis maps (see right bottom insert Fig.3.2 a)). In addition, a network of misfit dislocations along the substrate/film interface actuates the relaxation of the lattice mismatch strain within the first 2-3 monolayers of the growing film, yielding a strain-free state of the rest of the LPCMO film .

The temperature dependences of the resistivity, $\rho$, and magnetization, $M$, presented in Fig 3.3 , demonstrate extremely sharp and coupled metal-insulator $\left(T_{\mathrm{MI}}\right)$ and magnetic $\left(T_{\mathrm{C}}\right)$ transitions at $T_{\mathrm{MI}} \approx T_{\mathrm{C}}=195 \mathrm{~K}$, with the maximal value of logarithmic derivative of the resistivity, $\alpha_{\rho}=(T / \rho)(\mathrm{d} \rho / \mathrm{d} T)=140$. This confirms a high crystalline quality and chemical homogeneity of the films. The measured values of the residual resistivity, $\rho(4 \mathrm{~K}) \approx 180 \mu \Omega \mathrm{cm}$, and of the saturation magnetization, $M(10 \mathrm{~K}) \approx 600 \mathrm{emu} / \mathrm{cm}^{3} \approx 3.6 \mu_{\mathrm{B}} / \mathrm{Mn}$, (inset in Fig $3.3 \mathrm{~b}$ ) agree well with the nominal Ca-doping $x=0.3$. The zooms in $\rho(T)$ and $M(T)$ (insets to Figs.3.3a) and 3.3p)) reveal a warming/cooling hysteresis of $\Delta T \sim 1 \mathrm{~K}$, clearly seen for $T=185-195 \mathrm{~K}$, i.e. just below $T_{\mathrm{C}}$. Apparently, CMR $=100 \%(R(0)-R(4 \mathrm{~T})) / R(4 \mathrm{~T})$, as shown in Fig 3.3 a), develops exclusively within this narrow temperature window of about 

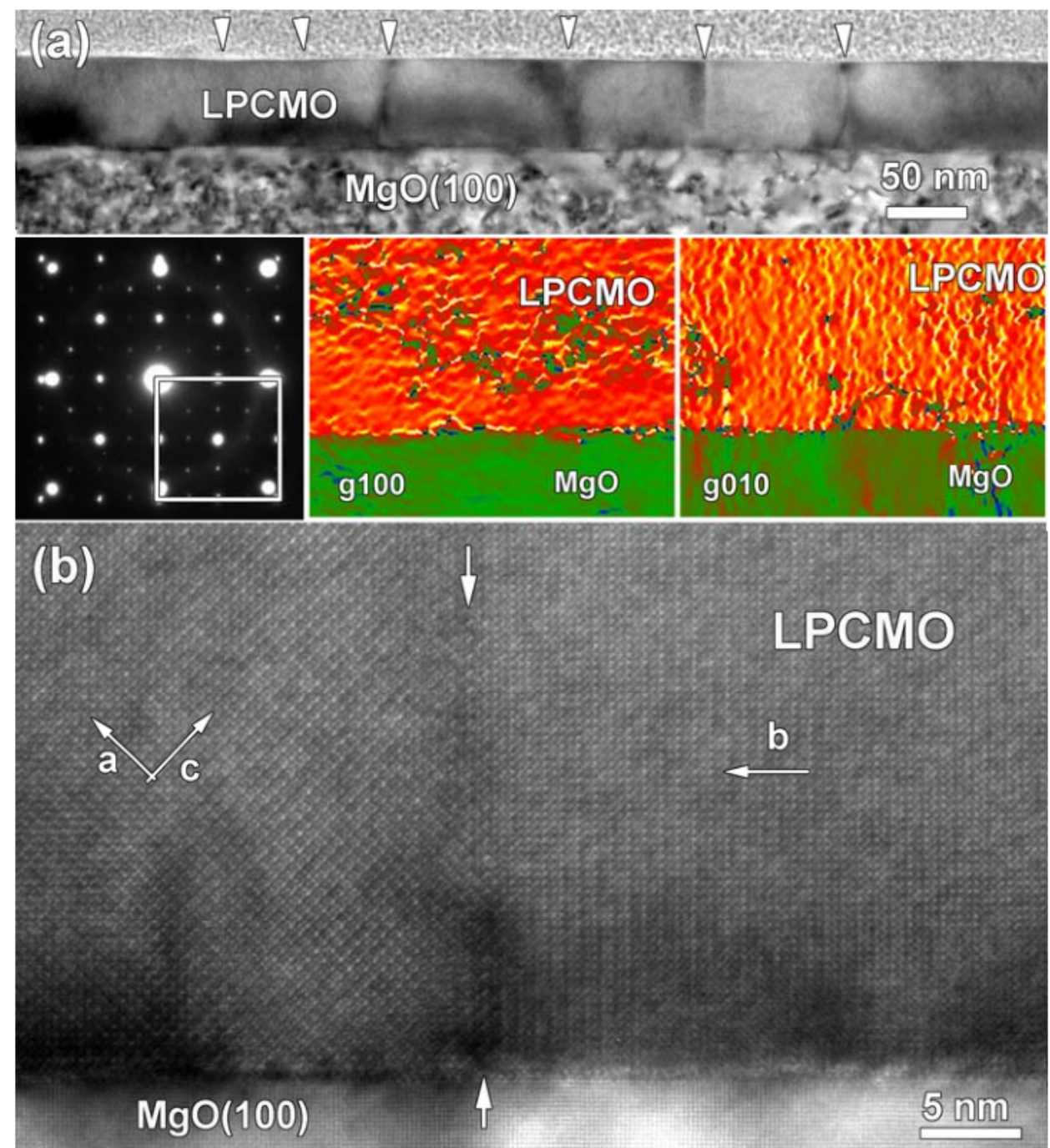

Figure 3.2.: a) Bright field low magnification TEM images of a LPCMO/MgO film and corresponding ED pattern showing a single crystalline epitaxial character of the film. Notice the presence of twin boundaries indicated by white arrowheads. An orthorhombic Pnma structure is indicated by diffraction spots $(1 / 2,0,0)$ and $(0,1 / 2,0)$ in ED pattern (left, bottom insert) and also twinning character of the film. HRTEM GPA color strain analysis maps along two orthogonal direction g100 (perpendicular to the interface) and g010 ( along the interface) are given as insert. Notice the absent of twin boundary in GPA pattern. (b) HRTEM image of a $\mathrm{LPCMO} / \mathrm{MgO}$ interface showing flat film/substrate interface and confirming a coexisting of crystallographic Pnma rotational twinning domains with heteroepitaxial perfect twin boundary indicated by white arrow. 
$10 \mathrm{~K}$. At low temperatures nor tunneling MR nor CMR was observed (see inset in Fig. 3.3 a) thus ruling out the grain-boundary-governed transport in the films under study. All this indicates that CMR in our LPCMO/MgO films is driven by the first order phase transition, as indicated by the hysteresis in $\rho(T)$ and $M(T)$, and can be viewed as a magnetic-field-induced insulator-to-metal transition.

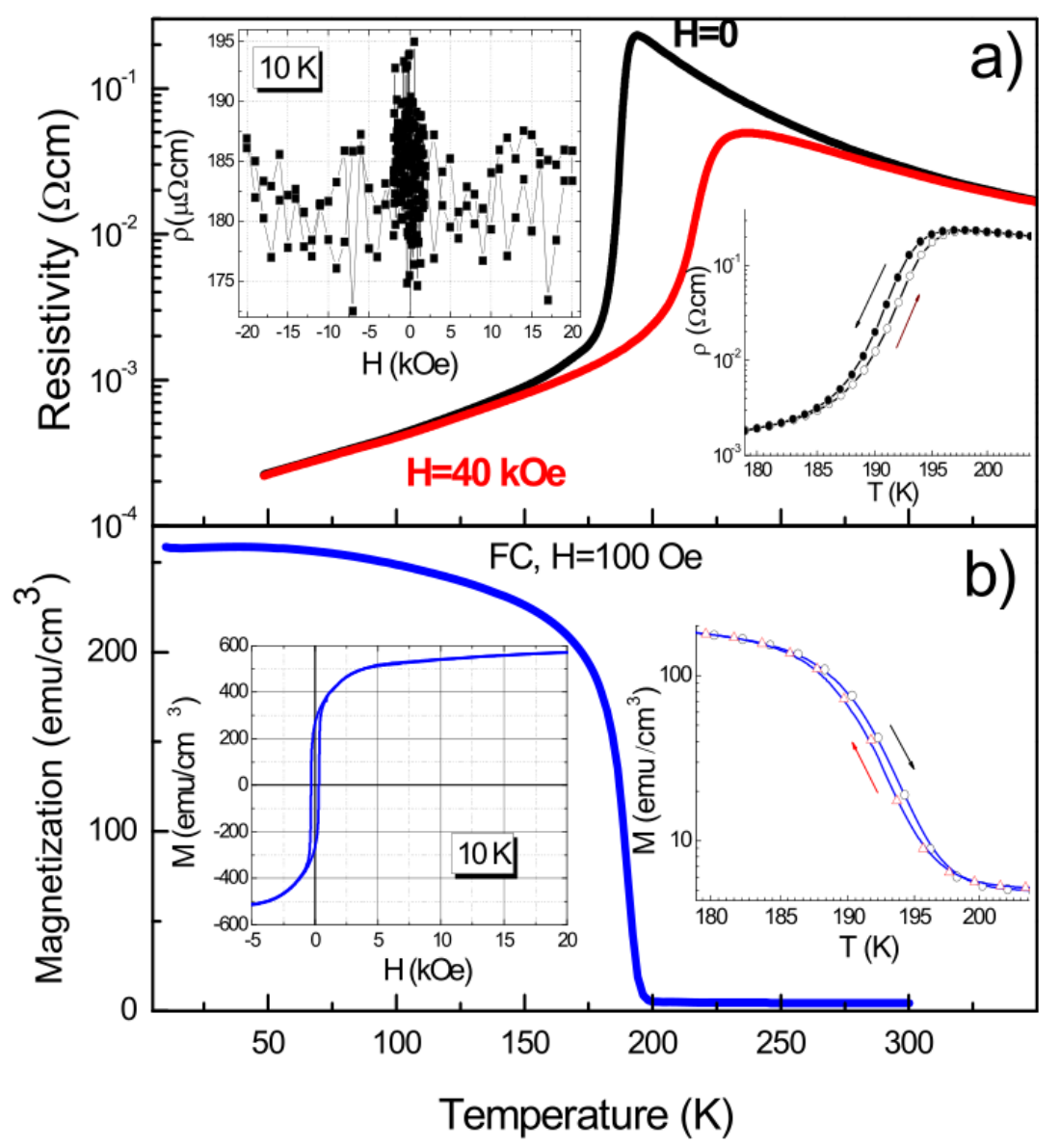

Figure 3.3.: General characteristics of the metal-insulator (a) and ferroparamagnetic (b) phase transitions in $\left(\mathrm{La}_{0.6} \mathrm{Pr}_{0.4}\right)_{0.67} \mathrm{Ca}_{0.33} \mathrm{MnO}_{3}$. The insets illustrate a temperature hysteresis in resistivity (a, right inset) and magnetization (b, right inset) as well as field dependences of the resistivity (a, left inset) and of the magnetization (b, left inset) at $T=10 \mathrm{~K}$.

In Fig 3.4 close correlation between the $\rho(H)$ and $M(H)$ dependences in the vicinity of phase transition is shown. For low fields, $0<H \leq 5 \mathrm{kOe}$, the resistivity (Fig.3.4a)) is hysteretic with two maxima at the coercive field $H_{c}$ (see the inset to Fig (3.4 A)). A suppression of CMR for $T<180 \mathrm{~K}$ is apparently accompanied by the vanishing of the $\rho(H)$ hysteresis. Furthermore, a magnetic ZFC-FC hysteresis, $\delta M=M(H \uparrow)-M(H \downarrow) \neq 0$, also "opens up" within the same temperature 
interval, $185 \mathrm{~K}<T<195 \mathrm{~K}$, and causes a dramatic increase of $H_{\text {sat }}$ (see inset in Fig 3.4 b)). Outside the phase transition region, both for the FM and paramagnetic $(\mathrm{PM})$ states, $M(H)$ and $\rho(H)$ curves are evidently not hysteretic. In the PM state for $200 \mathrm{~K}<T \leq 220 \mathrm{~K}$ a metamagnetic transition (Fig $3.4 \mathrm{~b}$ ) develops for $H \sim 5$-20 kOe and results in very large saturation magnetic moment, $M_{S}(205 \mathrm{~K})=5 \cdot 10^{-4} \mathrm{emu}$, which consists of $70 \%$ of the FM moment, $M_{S}(170 \mathrm{~K})=7 \cdot 10^{-4} \mathrm{emu}$. The $\rho(H)$ behavior (see Fig.3.4a)), being in close agreement with the metamagnetic transition, deviates strongly from the parabolic dependence, $\Delta \rho=\rho(H)-\rho(0) \sim-H^{2} \sim-M^{2}$, as would be the case for the field-induced PM magnetization [23]. For $T>220 \mathrm{~K}$ no metamagnetic transition was observed for $H \leq 50 \mathrm{kOe}$.
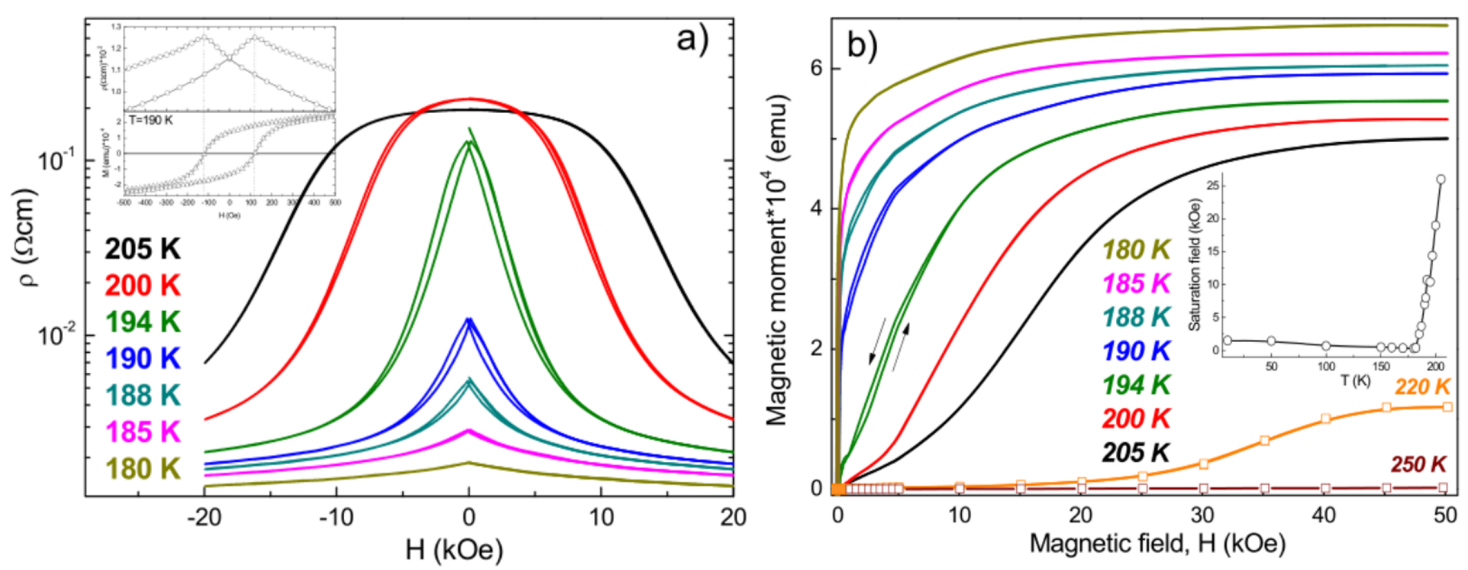

Figure 3.4.: Magnetic field dependencies of the resistivity (a) and magnetization (b) of an $\operatorname{LPCMO}\left(y=0.4, T_{\mathrm{C}}=195 \mathrm{~K}\right)$ film for temperatures, $180 \mathrm{~K}<T<205 \mathrm{~K}$, where the CMR develops. The inset to Fig. 2a) demonstrates the correlation between the coercive field (SQUID measurement) and field at which CMR shows a maximum. The inset to Fig. 2b) illustrates a strong enhancement of the saturation field in the vicinity of $T_{\mathrm{C}}$.

In Fig 3.5 the slow resistance relaxation, measured in the vicinity of the phase transition, is shown. After applying and switching off a magnetic field, $H=0-1 \mathrm{kOe}$, in addition to the instantaneous resistance change (CMR) we observed an exponential relaxation of the resistance, $R(t) \sim \exp \left(-t / \tau_{\text {res }}\right)$. Usually the slow resistance relaxation, along with the temperature- and high-field-driven $M(H)$ hysteresis, was considered as a manifestation of a metastable phase separated state [339, 340] close to the first order phase transition. In contrast to the previous experiments [339, 340, the slow resistance relaxation in LPCMO films, was observed for low magnetic fields $H<1$ kOe and, furthermore, $R(t)$ demonstrates the so called "recovery effect": after removal of magnetic field the resistance changes back to the initial value at $H=0$. The evaluated relaxation time, $\tau_{\text {res }} \approx 200 \mathrm{~s}$, can be modeled within the Neel relax- 

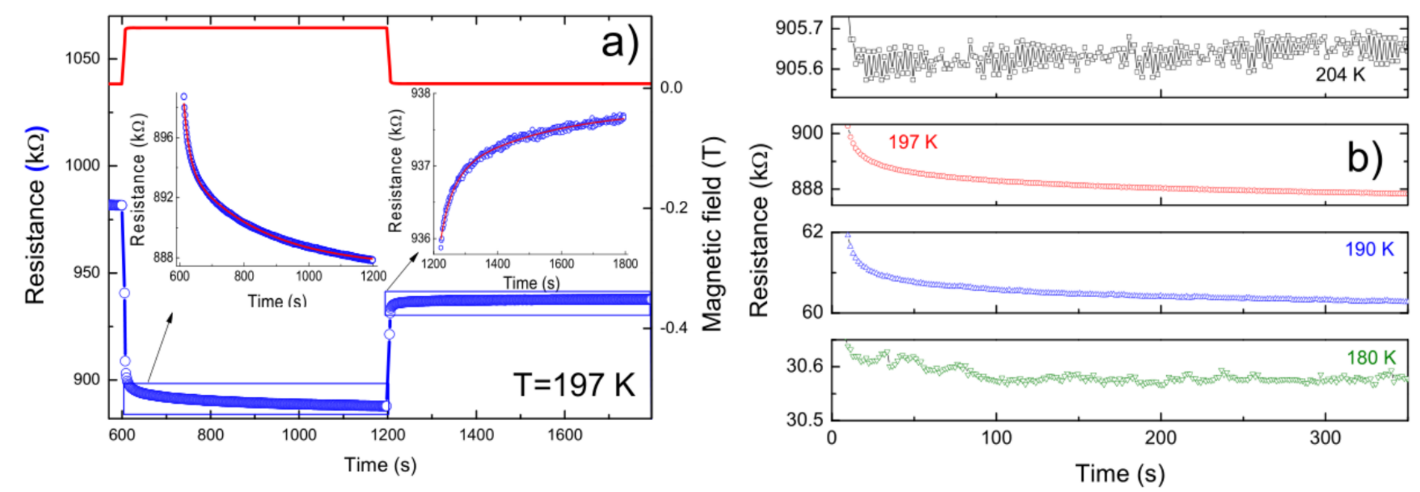

Figure 3.5.: a) Time dependences of the resistance in LPCMO film $(y=0.4)$ close to MI transition, measured after applying and switching off the external magnetic field, $H=1 \mathrm{kOe}$. Along with the instantaneous resistance changes, i.e. the CMR effect, one can see the "in-relaxation" i.e. the resistance decreases exponentially after applying the field and the "outrelaxation" (the resistance increases after switching off the field). The evaluated relaxation time $\tau \approx 200 \mathrm{~s} ; \mathrm{b}$ ) time dependences of the resistance after applying an in-plane magnetic field, $H=1 \mathrm{kOe}$, measured at different temperatures in the vicinity of $T_{\mathrm{C}}=195 \mathrm{~K}$.

ation of the magnetization (see below) and the super-paramagnetic model of Bean and Livingston 341. Importantly, the slow resistance relaxation is limited within the above discussed narrow temperature region close to $T_{\mathrm{C}}$ (see Fig. $3.5 \mathrm{~b}$ ) and is not present both for FM $\left(T \ll T_{\mathrm{C}}\right)$ and PM states.

In Fig.3.6a) we present the temperature dependences of the squared normalized coercive field, $h_{c}^{2}=\left(H_{c}(T) / H_{c}(0)\right)^{2}$, as a function of the normalized temperature, $t=\left(T-T_{\mathrm{C}}\right) / T$, evaluated from the magnetooptic and SQUID measurements of the LPCMO films with $y=0.375$ and 0.4 . At low temperatures $\left(T \ll T_{\mathrm{C}}\right)$ $h_{c}$ decreases with increasing temperature as expected for a FM material. The data can be fitted very well by the formula: $h_{c}=\left(1-T / T_{\mathrm{B}}\right)^{1 / 2}$, which describes a classic behavior of single-domain ferromagnetic particles [341]. The fitting parameter, $T_{B}=1.05 T_{C}$, is the so called blocking temperature, above which particles behave as an ideal superparamagnet $\left(h_{c}=0\right)$. The average radius of magnetic domains (particles) can be estimated from the expression $K_{\mathrm{U}} V / k_{\mathrm{B}} T_{B} \approx 25$, which is obtained from the Neel relaxation time, $\tau_{\text {obs }}(T)=\tau_{0} \exp \left(K_{\mathrm{U}} V / k_{\mathrm{B}} T_{B}\right)$, for the observable relaxation times, $\tau_{\text {obs }} \approx 100 \mathrm{~s}$, and $\tau_{0} \approx 10^{-9} \mathrm{~s}$ (see Ref.[341]). With the uniaxial anisotropy constant, $K_{\mathrm{U}}=3.6 \cdot 10^{4} \mathrm{~J} / \mathrm{m}^{3}$, measured for different manganites 342 344, including LPCMO, and the obtained $T_{\mathrm{B}}=220$ and $205 \mathrm{~K}$ for films with $y=0.375$ and 0.4 , respectively, the averaged radius of domains is $R_{\mathrm{RM}}=\left(75 k_{\mathrm{B}} T_{B} / 4 \pi K_{\mathrm{U}}\right)^{1 / 3} \approx 6-8 \mathrm{~nm}$. Such a small domain size illustrates 

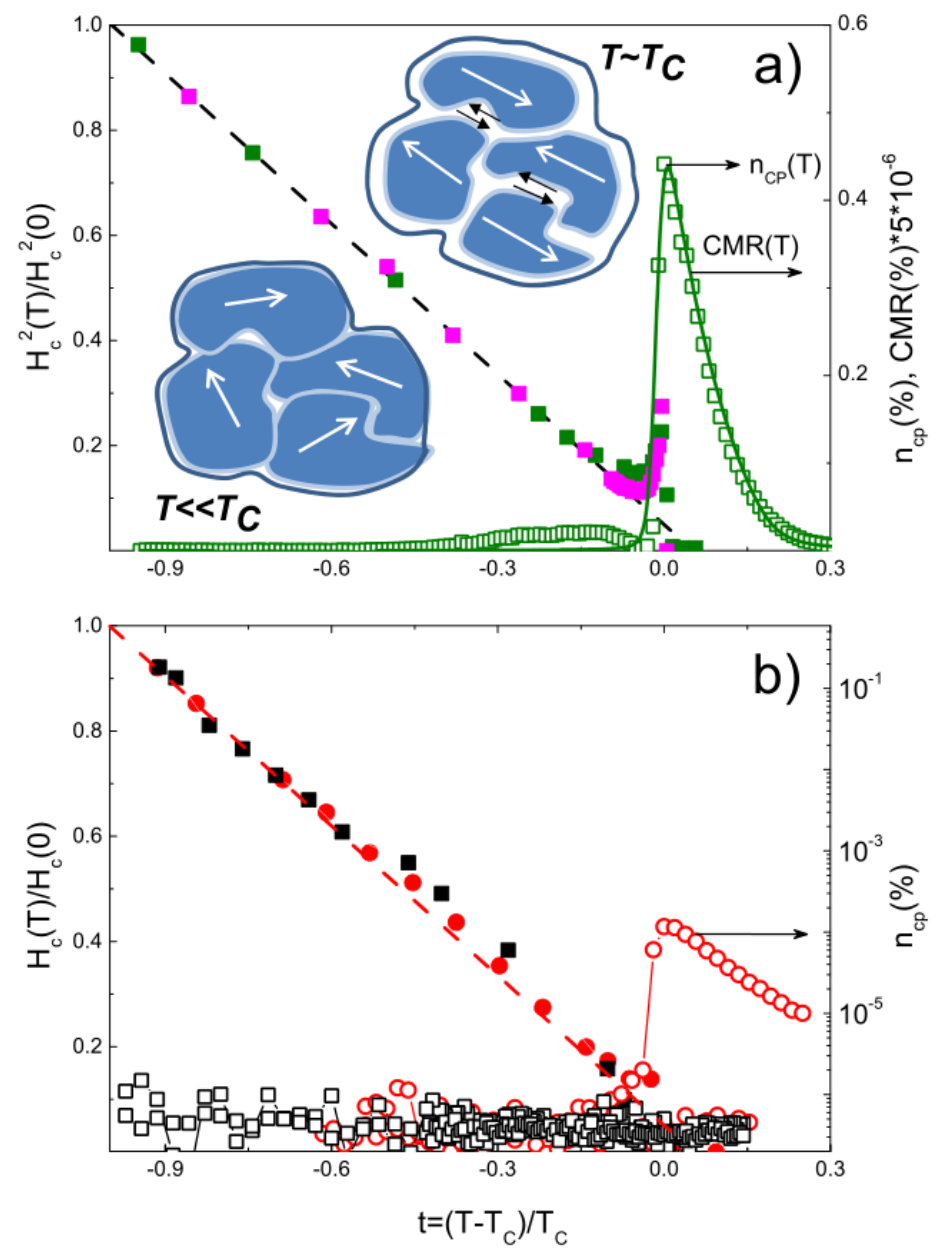

Figure 3.6.: a) The square of the measured normalized coercive field, $h_{c}^{2}=\left(H_{c}(T) / H_{c}(0)\right)^{2}$, as a function of the normalized temperature, $t=\left(T-T_{\mathrm{C}}\right) / T_{\mathrm{C}}$, for LPCMO films with $y=0.4$ (green) and $y=0.375$ (magenta) - left scale. The fit (dash line) is the dependence, $h_{c}^{2} \sim\left(1-t / t_{\mathrm{B}}\right)$, with the blocking temperature, $T_{\mathrm{B}}=1.05 T_{\mathrm{C}}$. On the right scale the temperature dependences of the relative amount of correlated polarons, $n_{\mathrm{CP}}(T)=U_{3 \omega}(T) / U_{\omega}(T)$, (open squares), and of $\operatorname{CMR}(T)$ (green line), multiplied by a factor of $5 \cdot 10^{-6}$ in LPCMO with $y=0.4$ demonstrate an enhancement in a number of polarons close to $T_{\mathrm{C}}$ as well as the scaling $n_{\mathrm{CP}}(T) \sim \operatorname{CMR}(T)$. In caption: the proposed magnetic structure near and far below $T_{\mathrm{C}}$; b) Normalized coercive field, $h=H_{c}(T) / H_{c}(0)$, as a function of normalized temperature, $t=\left(T-T_{\mathrm{C}}\right) / T_{\mathrm{C}}$, for optimally doped LCMO (red close circles) and LSMO (black close quadrates) films (left scale). One can see (the right scale) that $n_{\mathrm{CP}}(T)$ for LCMO (open red circles) and LSMO (open black squares) are by factor $10^{3}$ and $10^{5}$ smaller than that for LPCMO. 
a weakening of FM exchange and an increase of the electron-phonon coupling [20]. Moreover, by approaching $T_{\mathrm{C}}$ from below (see Fig $3.6 \mathrm{a}$ ) the coercive field, $h_{c}$, increases anomalously for LPCMO before vanishing for $T>T_{\mathrm{C}}$. This and the drastic increase of saturation field of the magnetization (Fig $3.4 \mathrm{p}$ ) are well-known fingerprints of exchange-coupled inhomogeneous magnetic systems. The classical examples are artificially layered systems: 1) $\mathrm{Co} / \mathrm{Cu} / \mathrm{Co}$ [345] with a nonmagnetic $\mathrm{Cu}$ layer, actuating a thickness dependent (AFM or FM) coupling of Ruderman-KittelKasuya-Yosida (RKKY) type; 2) Fe/ $\mathrm{Fe}_{1-x} \mathrm{Mn}_{x}$ bi-layers [346], with the FM layer (Fe) AFM coupled to the $\mathrm{Fe}_{1-x} \mathrm{Mn}_{x}(0.15<x<0.3)$ layer; and 3) oxide superlattices [347] of $\left(\mathrm{La}_{2 / 3} \mathrm{Ba}_{1 / 3} \mathrm{MnO}_{3} / \mathrm{LaNiO}_{3}\right)_{N}$. As shown for comparison in Fig. 3.6b), the $h_{c}(t)$ dependence in optimally doped $\mathrm{LCMO} / \mathrm{MgO}$ and $\mathrm{LSMO} / \mathrm{MgO}$ films is rather linear, pointing out the existence of weak-pinning centers [348]. Finally, no anomalous increase of coercive field close to $T_{\mathrm{C}}$ was observed both for LSMO and LCMO. Thus, the low-field hysteretic CMR in epitaxial LPCMO/MgO films close to the phase transition is intimately related to a peculiar magnetic state with FM nanodomains coupled to each other by AFM exchange. A hint to the possible origin of the exchange coupling mediator can be found in Fig.3.6a) (right scale), which demonstrates that $\operatorname{CMR}(T)$ scales nicely with the temperature dependence of electrical $3^{\text {rd }}$ harmonic coefficient, $K_{3 \omega}(T)=\log \left(U_{3 \omega}(T) / U_{\omega}(T)\right)$, here $U_{\omega}$ and $U_{3 \omega}$ are measured ac voltages at a fundamental $(\omega=117 \mathrm{~Hz})$ and third harmonic frequencies. As was discussed in details in Ref.[149], the origin of $3 \omega$-signal is ascribed to correlated polarons, which due to a CE-type ordering of $\mathrm{Mn}^{3+} / \mathrm{Mn}^{4+}$ can be considered as electric quadrupole moments. The latter are well-known sources of the nonlinear coupling to an electric field, $Q=\chi E^{2}$ (see Ref.[349]). The nonlinear resistance, $R_{3 \omega}=\mathrm{d} U_{3 \omega} / \mathrm{d} J$, being also proportional to the square of the current or $E$-field, provides then a measure of the number of quadrupoles, i.e. CPs, in the sample. The quotient $K_{3 \omega} \sim R_{3 \omega} / R_{\omega}$ is then proportional to the relative amount of CPs, $n_{\mathrm{CP}}=N_{\mathrm{CP}} / N_{0}$, to the whole number charge carriers, $N_{0}$, induced by Cadoping. Close to phase transition the amount of polarons 334 in LPCMO increases dramatically up to $n_{\mathrm{CP}} \approx 0.5 \%$ at $195 \mathrm{~K}$ and seems to be able to mediate the AFM coupling. Remarkably, the corresponding values of $K_{3 \omega}$ in LCMO and LSMO films (see Fig, 3.6b) are much smaller, i.e. about $10^{-4} \%$ and $10^{-7} \%$, respectively. These films compared to LPCMO reveal linear $h_{c}(t)$ dependences (see Fig.3.6p) as well as no anomalous increase of $h_{c}$ close to the phase transition. Apparently, the amount of correlated polarons in LCMO and LSMO is not sufficient for AFM coupling, and this seems to be the reason for the absence of low-field CMR as well as for moderate and very small CMR values for LCMO and LSMO films [149], respectively.

Another evidence for the enhancement of the polaronic phase at the phase transition could be obtained from Raman spectra, shown in Fig. 3.7. One can see characteristic temperature dependent Raman shifts at $440 \mathrm{~cm}^{-1}$ and $620 \mathrm{~cm}^{-1}$, identified [350, 351] as $E_{\mathrm{g}}$ (internal vibrations or bending) and $B_{2 \mathrm{~g}}$ (in-phase stretching) modes of $\mathrm{MnO}_{6}$ octahedra, respectively. Due to symmetry considerations $E_{\mathrm{g}}$ is one of five Raman active modes $\left(A_{1 \mathrm{~g}}+4 E_{\mathrm{g}}\right)$ of the rhombohedral $(\mathrm{R} \overline{\mathrm{c}} \mathrm{c})$ structure and $B_{2 \mathrm{~g}}$ 


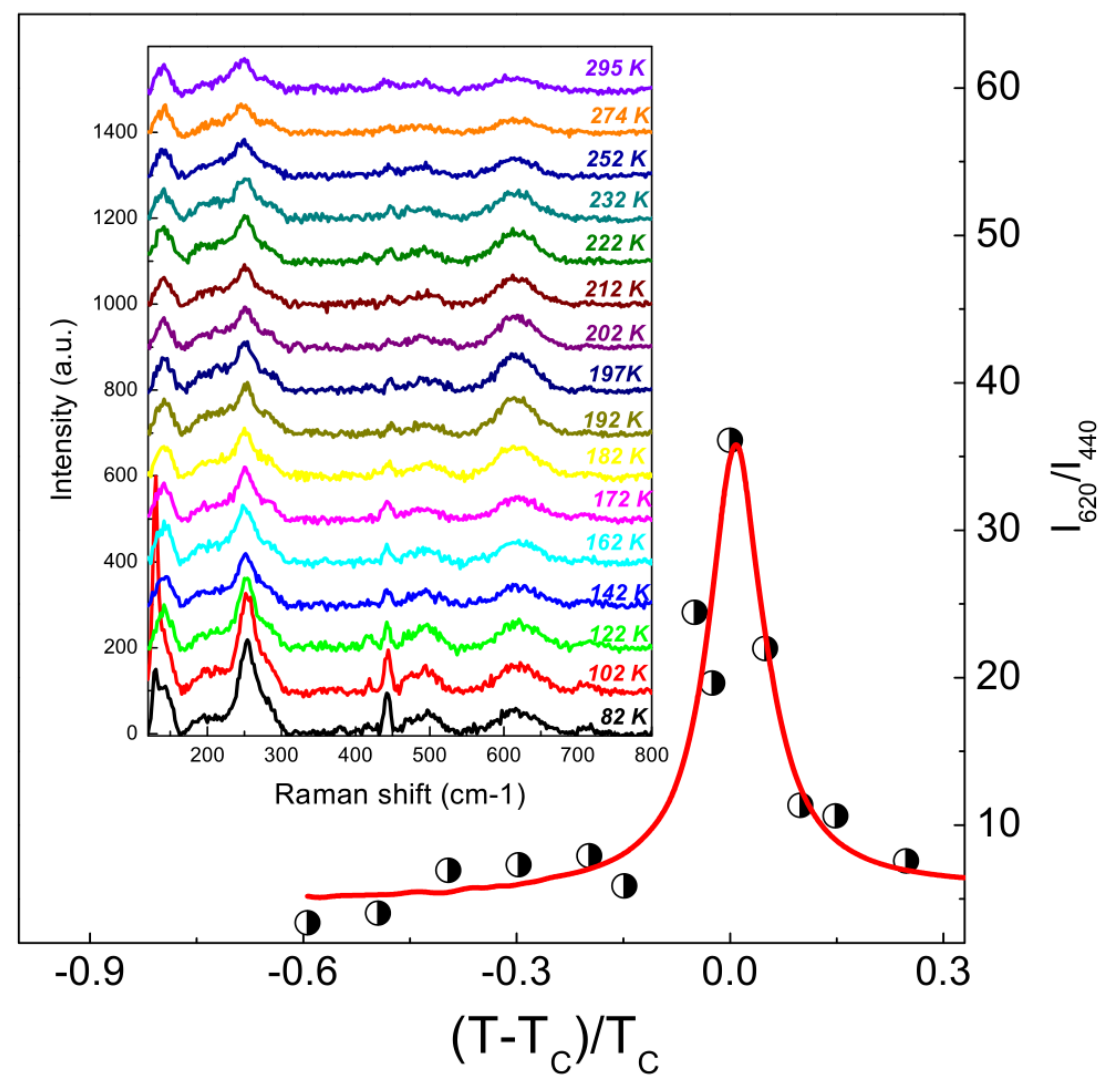

Figure 3.7.: The inset shows the temperature evolution of Raman spectra for LPCMO film $(y=0.4)$ for temperatures, $T=80-300 \mathrm{~K}$. Spectra were shifted by 100 units along vertical axis for clarity. The main panel demonstrates the temperature dependence of the intensity ratio between the Raman shift at $620 \mathrm{~cm}^{-1}$ due to JT phonons (stretching of $\mathrm{MnO}_{6}$ octahedra) and the Raman shift at $440 \mathrm{~cm}^{-1}$ (internal vibrations). A sharp peak close to $T_{\mathrm{C}}$ is in qualitative agreement with the behavior of correlated polarons in Fig. 3.6.

is the characteristic JT phonon mode within the orthorhombic (Pnma) structure, which has 24 active modes $\left(7 A_{1 \mathrm{~g}}+5 \mathrm{~B}_{1 \mathrm{~g}}+7 B_{2 \mathrm{~g}}+5 \mathrm{~B}_{3 \mathrm{~g}}\right)$. The intensity $I_{620}$ increases as the temperature decreases from $300 \mathrm{~K}$ down to $T_{\mathrm{C}} \approx 195 \mathrm{~K}$ and by further cooling this line becomes suppressed. In contrast, $I_{440}$ is suppressed by decreasing temperature down to $T_{\mathrm{C}}$, but it increases significantly in FM state for $T \ll T_{\mathrm{C}}$. The observed temperature evolution of Raman spectra reflects the competition between electron localization via JT distortions or CPs, which are compatible with a (Pnma) structure, and electron delocalization which favors a more symmetric (R $\overline{3} \mathrm{c}$ ) structure without JT distortions. The intensity ratio, $\gamma(T)=I_{620}(T) / I_{440}(T)$, (Fig. 3.7) illustrates this competition, underscoring a lattice aspect of the phase transition manifested by the enhancement of JT distortions at $T_{\mathrm{C}}$. 


\subsection{Discussion}

Remarkably, very different electric, $n_{\mathrm{CP}}(T)$, magnetic, $h_{c}(T)$, and structural, $\gamma(T)$, characteristics as well as $\operatorname{CMR}(T)$ itself, display a pronounced sharp peak close to the phase transition. This undoubtedly points out a strong coupling between electron, spin and lattice (phonons) degrees of freedom in an electronically/structurally inhomogeneous LPCMO. We consider this classic CMR material as an "intrinsic exchange coupled system", sketched in Fig.3.6a). Here, FM nanodomains are AFM exchange coupled (pinned) by correlated JT polarons (CPs), nucleated at the domain walls and assumed to possess AFM correlations. A preferred formation of the insulating $\mathrm{COO}$ phase at surfaces, interfaces or grain boundaries is well documented in the literature [227, 352, 353] and can be understood within the weakening of FM exchange at the two-dimensional defects. Due to a single crystalline character of our LPCMO/MgO(100) films (see structural data in Figs 3.1 3.2) and their relatively large thickness, $d \approx 50-70 \mathrm{~nm} \gg R_{\mathrm{FM}} \approx 6-8 \mathrm{~nm}$, grain boundaries and interfaces as locations points of CPs can be ruled out. Thus, domain walls provide a unique intrinsic possibility to host CPs. Phenomenological Ginzburg-Landau approaches predict the formation of a charge ordered [354 and AFM state [355] within the domain wall, especially when the bulk (domain) phase is located not far away from the FM/AFM boundary in the phase diagram [164]. Our LPCMO films [20] with Pr-doping, $y \approx 0.4$, and reduced $T_{\mathrm{C}}=195 \mathrm{~K} \gg 370 \mathrm{~K}$ in comparison to the double exchange FM metallic LSMO, fit nicely the above conditions to locate the AFM polaronic phase at the domain walls. The T-scale for low-field hysteretic CMR and for the underlying AFM coupling in LPCMO is limited from above by the appearance of charge ordering at $\mathrm{T} \mathrm{CO} \approx 220 \mathrm{~K}$ [152], which breaks the symmetry of PM phase and enhances the formation of CPs. Within the narrow temperature interval, $180 \mathrm{~K}<T_{\mathrm{C}}<T_{\mathrm{B}}<T_{\mathrm{CO}}$, a mixed phase with AFM layers and coupled FM nanodomains exists, yielding extremely large field-induced resistance changes and metamagnetic transition. Note a similarity between the blocking temperature, $T_{\mathrm{B}}<T_{\mathrm{CO}}$, and the $T^{*}$-scale [338, at which the "preformed magnetic clusters" (domains) can be substantially influenced by an applied magnetic field. A strain-free state of the LPCMO film and extremely sharp MI transition as an indicator for the absence of A-site quench disorder [334, both infer that such a mixed phase could be a true thermodynamic phase as was suggested earlier [354]. However, a more detailed inspection of the nature of mixed phase goes out of scope of this paper.

Our results, being in line with the electronic phase separation model of BURGY et al. [338], specify further the nature of competing phases, i.e. FM vs. AFM. They also provide evidence for the location of the AFM phase within the domain walls, where the FM order parameter becomes zero, changing from $+\mathrm{M}$ to $-\mathrm{M}$. Indeed, a polaronic AFM phase (Fig 3.6 a ) apparently resembles the "collinear" phase in Ref. 338] (Fig 3.2, "green phase"), which separates two domains with staggered "up" and "down" magnetizations. Remarkably, even a small amount of CPs can actuate the AFM coupling between FM nanodomains, thus, playing an important role 
in CMR. The maximal concentration of CPs in LPCMO, estimated from nonlinear electric measurements (see Fig.3.6 $)$, is $n_{\mathrm{CP}}\left(T_{\mathrm{C}}\right) \approx 0.5 \%$, and taking a homogeneous distribution of CPs with the size $\delta \approx 1.5 \mathrm{~nm}$ [144, 145], the average distance between them will be $A_{\mathrm{CP}}=\left(n_{\mathrm{CP}}\right)^{-1 / 3} \delta \approx 9 \mathrm{~nm}$. This fits nicely the size of FM domains, $R_{\mathrm{FM}} \approx 6-8 \mathrm{~nm}$, evaluated from magnetic measurements. Considering $A_{\mathrm{CP}}$ being a few nm large one can suggest an elastic (strain) mechanism [356] might play a role in actuating AFM coupling via correlated polarons. Mismatching lattice volumes of the polaronic, (Pnma), and polaron-free, $(\mathrm{R} \overline{3} \mathrm{c})$, phases may further support such strain scenario. For $T \ll T_{\mathrm{C}}$ in the globally FM metallic phase the amount of CPs in LPCMO is strongly reduced, $n_{\mathrm{CP}} \approx 10^{-6}$, and the distance between them becomes large, $A_{\mathrm{CP}} \approx 100 \mathrm{~nm} \ll R_{\mathrm{FM}}$, thus, allowing a strain relaxation and the absence of coupling.

The coupling constant, $J_{\mathrm{AFM}}$, can be estimated from the slow relaxation of resistivity (Fig.3.5), which actually means that after field removal the $J_{\mathrm{AFM}}$ acts as an effective field to restore the AFM coupling between FM nanodomains. For low magnetic fields, $H=0-1 \mathrm{kOe}$, and for $T \approx T_{\mathrm{C}}$, the resistance obeys a square field dependence, $\Delta R \sim-H^{2}$, but magnetization is a linear function of the field, $\Delta M \sim-H$. Taking $H$ as a parameter one gets $\Delta R \sim \Delta M^{2}$. Considering Neel relaxation of the magnetization, $\Delta M(t) \sim \exp \left(-t / \tau_{\text {mag }}\right)$, with magnetic relaxation time, $\tau_{\text {mag }}=\tau_{0} \exp \left(K_{\mathrm{U}} V / k_{\mathrm{B}} T_{\mathrm{B}}\right)$, one gets an exponential relaxation of the resistance, $\Delta R \sim \Delta M^{2} \sim \exp \left(-2 t / \tau_{\text {mag }}\right) \sim \exp \left(-t / \tau_{\text {res }}\right)$, with $\tau_{\text {res }}=\tau_{\text {mag }} / 2$. The relaxation time of the resistance in the presence of the Zeeman energy, $\mu \mu_{0} H$, and AFM coupling energy of the form, $E_{\mathrm{AFM}}=J_{\mathrm{AFM}} A$, can be written as: $\tau_{\text {res }}(T)=$ $\tau_{0} / 2 \cdot \exp \left(\left(K_{\mathrm{U}} V-\mu \mu_{0} H+E_{\mathrm{AFM}}\right) / k_{\mathrm{B}} T\right)$. For $T=197 \mathrm{~K}$, the magnetic moment of the domain, $\mu=n \mu_{\mathrm{B}}$, with $n$ is the number of Mn spins in the volume, $V=4 / 3 \pi\left(R_{\mathrm{FM}}\right)^{3}$, a magnetic field, $\mu_{0} H=0.1 \mathrm{~T}$, and the area, $A=4 \pi\left(R_{\mathrm{FM}}\right)^{2}$, of a domain, $R_{\mathrm{FM}} \approx 7 \mathrm{~nm}$, the AFM coupling constant, $J_{\mathrm{AFM}}=4.5 \cdot 10^{-4} \mathrm{~J} / \mathrm{m}^{2}$, and the AFM energy per Mnspin, $E_{\mathrm{AFM}} / \mathrm{Mn}=0.21 \mathrm{meV}=2.4 \mathrm{~K}$, were obtained. This coupling being much weaker than a typical AFM exchange energy, e.g. $10-12 \mathrm{meV}\left(T_{N} \approx 140 \mathrm{~K}\right.$ in $\mathrm{LaMnO}_{3}$ ), agrees with a short-range character of the COO polaronic phase. The value estimated above exceeds the interlayer coupling, $0.1 \mathrm{erg} / \mathrm{cm}^{2}=10^{-4} \mathrm{~J} / \mathrm{m}^{2}$, observed in artificial FM/AFM exchange-bias manganite heterostructures [357], but is comparable with the RKKY coupling in oxide superlattices [347], $J_{\mathrm{RKKY}} \approx 3 \cdot 10^{-4} \mathrm{~J} / \mathrm{m}^{2}$. Such system with intrinsic AFM coupling, originated from a phase coexistence, is an insulator, which shows a resistivity maximum at $H \approx H_{c}$ due to the antiparallel orientation of the magnetizations of adjacent nanodomains. For fields, $H_{c}<H<H_{\text {sat }}$, the magnetization of the nanodomains flips along the field direction, but the "interface" spins still remain AFM coupled, yielding the hysteretic $\rho(H)$ and $M(H)$ behavior (Fig $3.2 \mathrm{a}$ ) and $3.2 \mathrm{~b})$ ). Larger fields, $H>H_{\text {sat }} \approx 10-30 \mathrm{kOe}$, finally destroy the AFM correlations, resulting in a closure of the $\rho(H)$ and $M(H)$ curves. For the FM state the resistance contribution due to spin scattering of the charge carriers at the domain wall, $\rho \sim \cos \left(\Theta_{i j}\right)$, becomes small. Indeed, in the absence of AFM coupling the angle, $\Theta_{i j}$, between magnetizations of adjacent domains de- 
creases. As a result epitaxial LPCMO films show a typically low residual resistivity, value, $\tau_{\text {res }}(4.2 \mathrm{~K}) \approx 10^{-4} \Omega \mathrm{cm}$, similar to that of the double-exchange LSMO [23], which contains no CPs. One can see a striking analogy between the observed intrinsic low-field CMR in LPCMO films close to $T_{\mathrm{C}}$ and the extrinsic interfacial low-field tunneling magnetoresistance (TMR) in granular manganites [357] or in the LSMO/STO/LSMO trilayers [358]. They both show two resistance maxima at $\pm H_{c}$ originating from AFM coupling between FM metallic domains (CMR) or electrodes (TMR). However, in the TMR systems a symmetry break of the $\mathrm{MnO}_{6}$ network at the interface leads [350] to the orbital reconstruction at the interface, stabilizing a highly isolating COO-CE phase in very thin manganite films [351] and manganite/titanite superlattices [352]. In contrast, the $\mathrm{MnO}_{6}$ frame remains continuous in an intrinsic CMR system and CPs, nucleated at the interfaces between FM domains, show presumably short-range CO-AFM correlations, which can be destroyed at relatively low magnetic fields of few $\mathrm{kOe}$. Interesting for applications is the fact that low-field CMR was observed at relatively high temperatures $\approx 200 \mathrm{~K}$, indicating a persistence of high spin polarization in FM domains close to $T_{\mathrm{C}}$.

\subsection{Conclusion}

CMR in epitaxial strain-free $\left(\mathrm{La}_{0.6} \mathrm{Pr}_{0.4}\right)_{0.67} \mathrm{Ca}_{0.33} \mathrm{MnO}_{3} / \mathrm{MgO}$ films is demonstrated to be a low-field and hysteretic effect, controlled by peculiar magnetic domain structure with FM nanodomains, AFM coupled by correlated Jahn-Teller polarons nucleated at the domain walls. The obtained small size of FM nanodomains, $R_{F M} \approx 6$ $8 \mathrm{~nm}$, taken together with the estimated coupling constant, $J_{\mathrm{AFM}}=4.5 \cdot 10^{4} \mathrm{~J} / \mathrm{m}^{2}$, provide a rational explanation of the long-standing issue in CMR physics, i.e. how a tiny amount of correlated polarons can be responsible for extremely large magneticfield-induced resistance changes.

\subsection{Acknowledgment}

The authors thank Prof. W. Felsch for fruitful comments and discussions, and Prof. A. L. Chuvilin for FIB specimen preparation. Financial support from the Deutsche Forschungsgemeinschaft (DFG) via SFB 602 (TP A2) and SFB 1073 (TP B1 and B4), the Leibniz Program and EU FP7 (IFOX Project) is acknowledged. K.Y.C acknowledges financial support from the Humboldt Foundation and the NRF of Korea (No. 2009-0093817). 


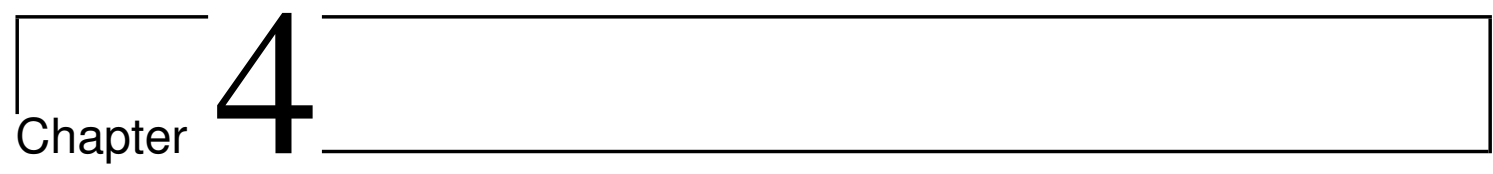

\section{Publication 2}

\section{Magnetic-Field-Induced Suppression of Jahn-Teller Phonon Bands in $\left(\mathrm{La}_{0.6} \mathbf{P r}_{0.4}\right)_{0.7} \mathrm{Ca}_{0.3} \mathrm{MnO}_{3}$ : the Mechanism of Colossal Magnetoresistance shown by Raman spectroscopy}

S. Merten ${ }^{1}$, O. Shapoval ${ }^{2}$, B. Damaschke ${ }^{1}$, K. Samwer ${ }^{1}$ and V. Moshnyaga ${ }^{1}$

${ }^{1}$ I. Physikalisches Institut, Georg-August University Göttingen, Friedrich-Hund-Platz 1, D-37077, Göttingen

2 IIEN, Academy of Sciences of Republic Moldova, Strada Academiei 3/3, MD-2028, Chisinau, Republic of Moldova.

Scientific Reports 9, 2387 (2019)

DOI: $10.1038 / \mathrm{s} 41598-019-39597-1$

Accepted for publication on January $28^{\text {th }}, 2019$

Published online February $20^{\text {th }}, 2019$

Licensed under a Creative Commons Attribution 4.0 International license (CC BY 4.0). 


\subsection{Motivation and Summary}

The huge number of Raman studies of the last decades demonstrated the enormous capabilities to study the polaronic behavior in manganites by Raman spectroscopy. Although temperature-dependent measurements could be successfully correlated with the temperature-dependent linear and nonlinear resistivity as presented in the previous chapter [66], studies in the CMR regime, i.e. magnetic-field-dependent Raman studies of mixed-valence CMR manganites, were still missing at the beginning of this thesis. Only one study of $\mathrm{La}_{0.25} \mathrm{Pr}_{0.375} \mathrm{Ca}_{0.375} \mathrm{MnO}_{3}$ [60] dealt with the magneticfield-dependent melting of the charge- and orbital-ordered phase. Therefore, to clarify the polaronic contribution to the CMR, we performed a magnetic-field-dependent Raman study on the prototypical CMR material, $\left(\mathrm{La}_{0.6} \mathrm{Pr}_{0.4}\right)_{0.7} \mathrm{Ca}_{0.3} \mathrm{MnO}_{3}$, in a range of $\mathrm{H}=0-50 \mathrm{kOe}$. The publication based on these measurements will be presented in the following section.

In the vicinity of the Curie temperature, $T_{\mathrm{C}}=197 \mathrm{~K}$ in this case, the intensity of the Jahn-Teller stretching mode at $614 \mathrm{~cm}^{-1}$ and of the bending mode at $443 \mathrm{~cm}^{-1}$ was found to be suppressed and enhanced, respectively. The observed magneticfield-induced intensity behavior $\Delta I$ shows a qualitative resemblance with the field and temperature dependence of the CMR of the same compound. In the case of the JT stretching vibrations, the suppression of the Raman intensity is even in quantitative agreement with the $\mathrm{CMR}$ effect $(\Delta I \approx 6000 \%, \mathrm{CMR}=7000 \%)$. This work provides, therefore, direct evidence that the reduction of the number of JahnTeller polarons at the phase transition is the main mechanism underlying the CMR.

\subsection{Introduction}

The rediscovering of the colossal magnetoresistance (CMR), i.e. a drastic decrease of electrical resistance in an applied magnetic field, in thin mixed-valence manganite films [21, 22] initiated an enormous boom of fundamental and applied research. For over twenty years, the long-standing important issue in CMR physics is the role of electron-phonon coupling and the mechanisms of electron-lattice correlations, controlling the coupled magnetic and metal-insulator transitions. In systems with strong electron-phonon coupling [164], like $\left(\mathrm{La}_{1-y} \mathrm{Pr}_{y}\right)_{1-x} \mathrm{Ca}_{x} \mathrm{MnO}_{3}$, a pronounced nm-scale coexistence with ferromagnetic (FM) metallic and charge-ordered insulating (COI) phases [152] and extremely large CMR $\left(\mathrm{CMR} \approx 10^{5}-10^{7} \%\right)$ were observed. In the parent compound $\mathrm{LaMnO}_{3}$ (LMO), the Jahn-Teller (JT) effect solely determines the structure and magnetism by means of a cooperative ordering of JahnTeller distorted $\mathrm{MnO}_{6}$ octahedra. In doped manganites, like $\left(\mathrm{La}_{0.6} \mathrm{Pr}_{0.4}\right)_{0.7} \mathrm{Ca}_{0.3} \mathrm{MnO}_{3}$ (LPCMO), only short-range static JT distortions, dubbed as correlated polarons [145] (CPs), exist and contribute to the phase separation. CPs are viewed as charge/orbital ordered (COO) lattice superstructures of the CE type and with antiferromagnetic $(\mathrm{AFM})$ correlations at a length scale, $d_{\mathrm{CP}} \approx 1-2 \mathrm{~nm}$, revealed by 
neutron and x-ray scattering [144, 146]. Recently, we have shown that even a tiny amount of CPs plays a key role in CMR as they are able to mediate an intrinsic AFM coupling between the nm-size FM domains, yielding an increase of the resistivity at the metal-insulator transition for antiparallel orientations of FM nano-domains at the coercive field $\mathrm{H}_{c}$ [66]. Furthermore, MicheLmann et al. observed a softening of the bulk modulus near $T_{\mathrm{C}}$, which can be attributed to the strong electron-phonon coupling in the $\left(\mathrm{La}_{0.6} \mathrm{Pr}_{0.4}\right)_{0.7} \mathrm{Ca}_{0.3} \mathrm{MnO}_{3}$ (LPCMO) system and thus, the presence of correlated polarons [150]. The origin and architecture of correlated polarons as short-range JT distortions makes it possible to probe them by Raman spectroscopy and to monitor their behavior across the phase transition. For the orthorhombic structure, e.g. LMO, the two high frequency modes at $490 \mathrm{~cm}^{-1}$ and $611 \mathrm{~cm}^{-1}$ are of particular interest, since they are directly related to the coherent JT distortion in the system [58]. These Raman features are forbidden in rhombohedral manganites, possessing no static JT distortions. Upon doping with divalent alkaline earth elements, like $\mathrm{Ca}^{2+}$, an oxygen disorder is introduced into the $\mathrm{MnO}_{6}$ octahedral network of LMO and thus, instead of a coherent, the incoherent JT distortions are formed. This leads to a broadening of the JT modes due to a disorder-induced phonon scattering and can be qualitatively described by means of the phonon density of states (PDOS) [65]. Temperature-dependent Raman spectra of $\left(\mathrm{La}_{0.7} \mathrm{Ca}_{0.3} \mathrm{MnO}_{3}\right.$ [65, 258] have shown a remarkable interplay between the broad JT stretching mode around $600 \mathrm{~cm}^{-1}$ and a sharp bending mode around $438 \mathrm{~cm}^{-1}$ : the intensity of the former is suppressed and of the latter is increased below $T_{\mathrm{C}}$. According to the disorder-order scenario of the phase transition, proposed by ILIEv et al. [65], the average lifetime, $\tau_{h}$, of a $\mathrm{Mn}^{3+}$ state in the paramagnetic state $\left(T>T_{\mathrm{C}}\right)$, i.e. the time between two consecutive $\mathrm{Mn}^{3+} \rightarrow \mathrm{Mn}^{4+}$ hopping events, is larger than the average lifetime of the JT distortion, $\tau_{J T}$. Hence, a quasi-static JT distortion develops yielding the broadening of the Raman mode. In the FM metallic state $\left(T<T_{\mathrm{C}}\right)$, in turn, $\tau_{h} \ll \tau_{J T}$, resulting in a suppression of the JT mode, since no more $\mathrm{Mn}^{3+}$ ions can exist. The arising sharp bending mode corresponds to a $\Gamma$-point phonon of a more ordered structure so that, the insulator-metal and paramagnetic-to-ferromagnetic transition is accompanied by a disorder-order transition in the $\mathrm{MnO}_{6}$ octahedral network. To the best of our knowledge, no magnetic-field-dependent Raman studies of the optimally doped CMR materials, e.g. LPCMO, were reported up to now. One can expect that in such a material a similar effect will occur in an applied magnetic field at $T \approx T_{\mathrm{C}}$. A previous magnetic-field-dependent Raman study was focused on the melting of the long-range-ordered $\mathrm{COO}$ insulating phase in $\left(\mathrm{La}_{1-y} \mathrm{Pr}_{y}\right)_{1-x} \mathrm{Ca}_{x} \mathrm{MnO}_{3}$ $(y=0.6, x=0.375)$ [60]. However, in contrast to LPCMO, the magnetic-fieldinduced melting of the $\mathrm{COO}$ phase is accompanied by a structural phase transition from a monoclinic $\left(\mathrm{P} 2{ }_{1} / \mathrm{m}\right)$ to the orthorhombic (Pnma) structure.

Here, we report a magnetic-field-dependent Raman study in thin films of a classic CMR material $\left(\mathrm{La}_{0.6} \mathrm{Pr}_{0.4}\right)_{0.7} \mathrm{Ca}_{0.3} \mathrm{MnO}_{3}$ and discuss it in terms of the disorder-order phase transition driven by magnetic field. The magnetic-field-induced suppression of the JT mode and an intensity gain of the bending mode was observed in the 
vicinity of $T_{\mathrm{C}}$, demonstrating the key role of the correlated JT polarons in the CMR effect.

\subsection{Results \& Discussion}

Structural, electrical and magnetic characterization of the LPCMO film (see supplementary information S1 in appendix A has revealed a coupled metal-insulator and ferromagnetic-paramagnetic phase transition at $T_{\mathrm{C}}=197 \mathrm{~K}$. In Fig 4.1, the unpolarized Raman spectra, measured in applied magnetic fields, $H=0-50 \mathrm{kOe}$, are shown for (a) $T \ll T_{\mathrm{C}}$ (b) $T=T_{\mathrm{C}}$ and (c) $T>T_{\mathrm{C}}$. The electronic background continuum was modeled by a collision-limited model [249] and the phonon peaks were fitted by multiple Lorentzian line shapes. As an example the best fit curve and the different components are shown in Fig. 4.2 (see also the supplemental information S2 in appendix A. The agreement between the experimental Raman spectra and the fitting curves were quite good at all analyzed temperatures and magnetic fields $\left(\mathrm{R}^{2} \approx 0.99\right)$. Furthermore, no signs of a Fano line shape were observed at any temperature or applied magnetic field, indicating no interaction between the electronic background continuum and the phonons [247]. The Raman spectra of LPCMO consist of seven phonon modes characteristic for manganites with orthorhombic structure e.g. $\mathrm{La}_{0.7} \mathrm{Ca}_{0.3} \mathrm{MnO}_{3}$ [65] or undoped orthorhombic $\mathrm{LaMnO}_{3}$ [58, 59]: rotational modes at $245 \mathrm{~cm}^{-1}\left(A_{1 \mathrm{~g}}, \omega_{1}\right)$ and $280 \mathrm{~cm}^{-1}\left(A_{1 \mathrm{~g}}, \omega_{2}\right)$, a displacement of the oxygen ion at $360 \mathrm{~cm}^{-1}\left(B_{2 \mathrm{~g}}, \omega_{3}\right)$, bending modes at $424 \mathrm{~cm}^{-1}\left(A_{1 \mathrm{~g}}, \omega_{4}\right)$ and $443 \mathrm{~cm}^{-1}\left(B_{2 \mathrm{~g}}, \omega_{\text {bend }}\right)$ as well as the JT stretching modes at $492 \mathrm{~cm}^{-1}\left(A_{1 \mathrm{~g}}, \omega_{\mathrm{as}}\right)$ and at $614 \mathrm{~cm}^{-1}\left(B_{2 \mathrm{~g}}, \omega_{\mathrm{s}}\right)$, respectively. The high frequency electronic (HFE) contribution at $\approx 900 \mathrm{~cm}^{-1}$ was assigned to the photoionization of small polarons [249]. For $T>T_{\mathrm{C}}$ the stretching mode $\left(\omega_{\mathrm{s}}\right)$ shows a small shoulder and splits visibly into two modes at $614 \mathrm{~cm}^{-1}$ (intrinsic) and $630 \mathrm{~cm}^{-1}$ (extrinsic) for temperatures $T<T_{\mathrm{C}}$ and magnetic fields, $H>20 \mathrm{kOe}$, respectively. This additional mode at $\approx 630 \mathrm{~cm}^{-1}\left(\omega_{\mathrm{N} 1}\right)$ was also observed in a thickness-dependent Raman study on $\mathrm{LaMnO}_{3}$ and is assigned to the film/substrate interface [359], pointing out its extrinsic origin.

In the following, we will focus on the magnetic field behavior of the stretching mode $\omega_{\mathrm{s}}$ as well as of the bending mode $\omega_{\text {bend }}$, because of their interplay at the temperature-driven phase transition [65, 66]. First, we will look at the Raman spectra measured far away from the transition temperature $T_{\mathrm{C}}$, shown in Fig 4.1 a and 4.1k. In the insulating paramagnetic state, $T>T_{\mathrm{C}}$, an applied magnetic field has no influence on the phonon modes. A similar behavior is observable for temperatures $T \ll T_{\mathrm{C}}$, i.e. deep in the FM metallic state. Therefore, we conclude that deep in the insulating and metallic state, the phonon and the electron [66] system of LPCMO is stable against an external magnetic field. In contrast, in the vicinity of $T_{\mathrm{C}}$ (Fig $4.1 \mathrm{~b}$ ), one can see a strong suppression of the JT modes and a less pronounced intensity increase of the bending mode $\omega_{\text {bend }}$ is observable. Note that even at high magnetic fields, i.e. $H=50 \mathrm{kOe}$, remnants of the JT modes are still 


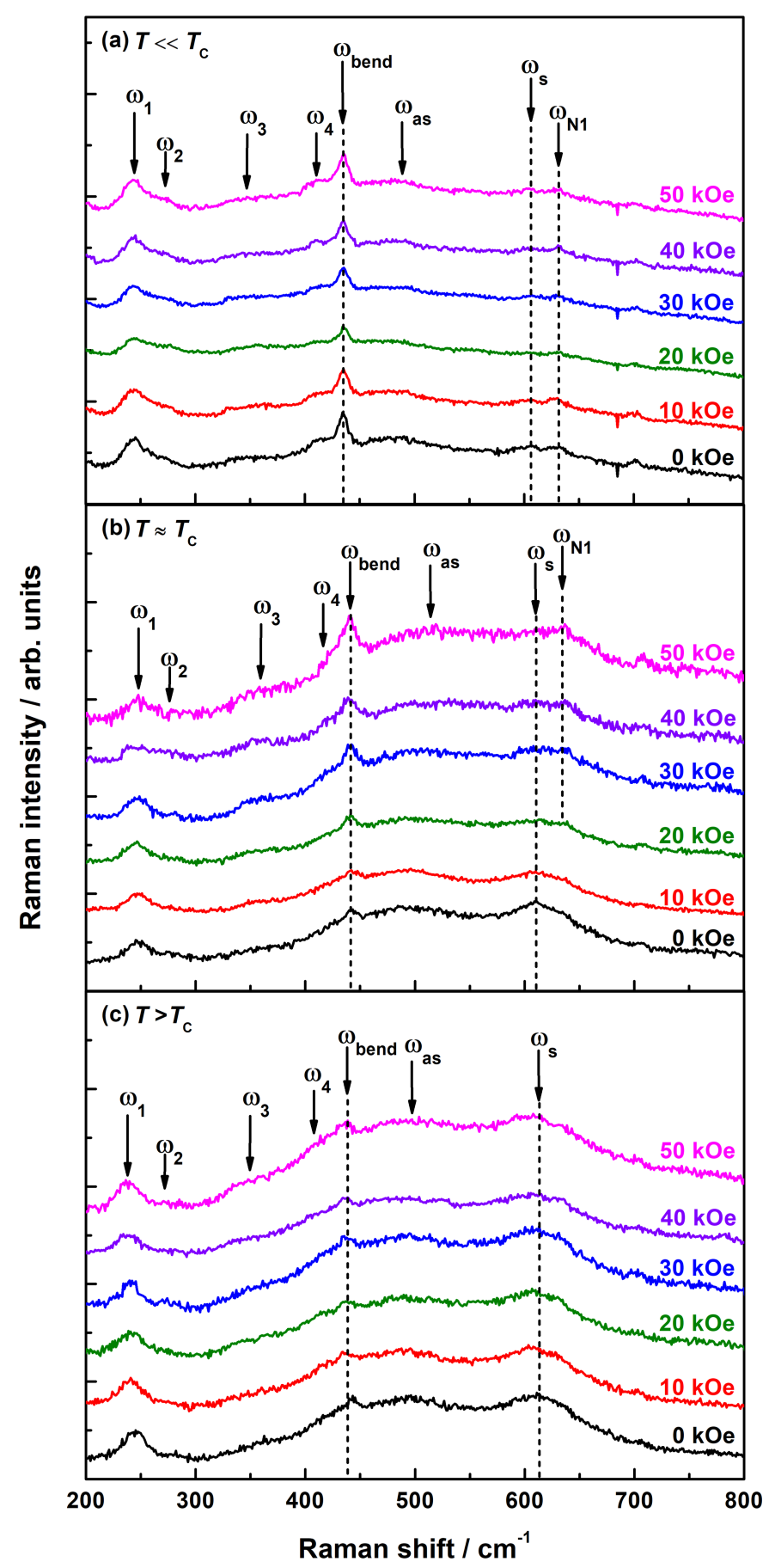

Figure 4.1.: Magnetic-field-dependent Raman spectra of LPCMO measured at different temperatures: (a) $T=90 \mathrm{~K} \ll T_{\mathrm{C}}$ (b) $T=T_{\mathrm{C}}=195 \mathrm{~K}$ (c) $T=245 \mathrm{~K}>T_{\mathrm{C}}$. The dashed lines indicate the Raman modes of interest, the bending mode $\omega_{\text {bend }}$ and the JT stretching mode $\omega_{\mathrm{s}}$ as well as the extrinsic JT mode $\omega_{\mathrm{N} 1}$. 
visible supporting the model of electronic phase separation [65, 66, 152]. In addition, the above mentioned splitting of the $\omega_{\mathrm{s}}$ mode can also be seen at $T=T_{\mathrm{C}}$ and magnetic fields $H>20 \mathrm{kOe}$. It becomes even clearer due to the progressive suppression of the intrinsic JT mode and the less sensitive field behavior of the extrinsic one.

To illustrate the close relation of the $\omega_{\mathrm{s}}$ and bending mode $\omega_{\text {bend }}$ to the phase transition, one can summarize their field behavior as the relative change of the intensities of the corresponding Raman modes in a magnetic field as a function of temperature, i.e. in a $\Delta I(T, 50 \mathrm{kOe})=100 \%|(I(50 \mathrm{kOe})-I(0 \mathrm{kOe})) / I(50 \mathrm{kOe})|$ vs. $\Delta T=T-T_{\mathrm{C}}$ plot as shown in Fig 4.3 a. One can see that the $\Delta I_{j}(T, 50 \mathrm{kOe})$ behavior of these modes show a remarkable qualitative and, in the case of $\omega_{\mathrm{s}}$, even a quantitative similarity to the CMR effect (see Fig.4.3p). Indeed, the CMR and $\Delta I_{j}(T, 50 \mathrm{kOe}$ ) are maximal only in the vicinity of $T_{\mathrm{C}}$. Note that, the intrinsic JT mode shows much higher field sensitivity than the bending mode. The relative change of $\omega_{\mathrm{s}}$ is negative, but since the $\Delta I_{j}(T, 50 \mathrm{kOe})$ is defined as the absolute value, the sign is not reflected in the $\Delta I(T, 50 \mathrm{kOe})$ value. Quantitatively, the observed $\Delta I_{\mathrm{s}}(T, 50 \mathrm{kOe}) \approx 6 \cdot 10^{3} \%$ of the intrinsic JT mode is of the same order of magnitude as the CMR $\approx 7 \cdot 10^{3} \%$, whereas $\omega_{\text {bend }}(T, 50 \mathrm{kOe}) \approx 65 \%$ is by about two orders of magnitude smaller (see Fig.4.3 a). Moreover, one can also see a difference of the linewidth of the $\Delta I_{\mathrm{s}}(T, 50 \mathrm{kOe})$ and the CMR: the former shows an extremely sharp maximum close to $T_{\mathrm{C}}$ and the latter is significantly broader for $T>T_{\mathrm{C}}$. Maybe, this is related to the difference in the probed scale as the CMR was measured on the macroscopic mm-

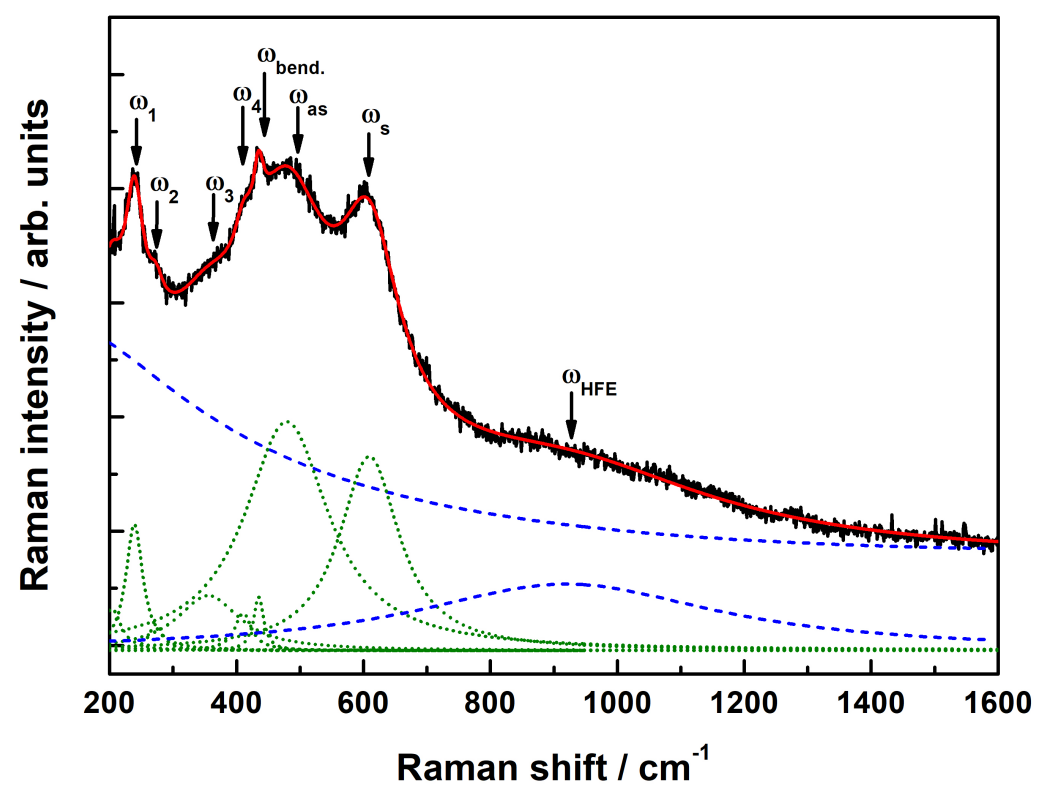

Figure 4.2.: The Raman spectrum at $245 \mathrm{~K}$ of LPCMO and the best fit curve (red) are shown. The phonon contribution (dotted green) and the electronic contributions (dashed blue) are shown separately. 
Temperature / K

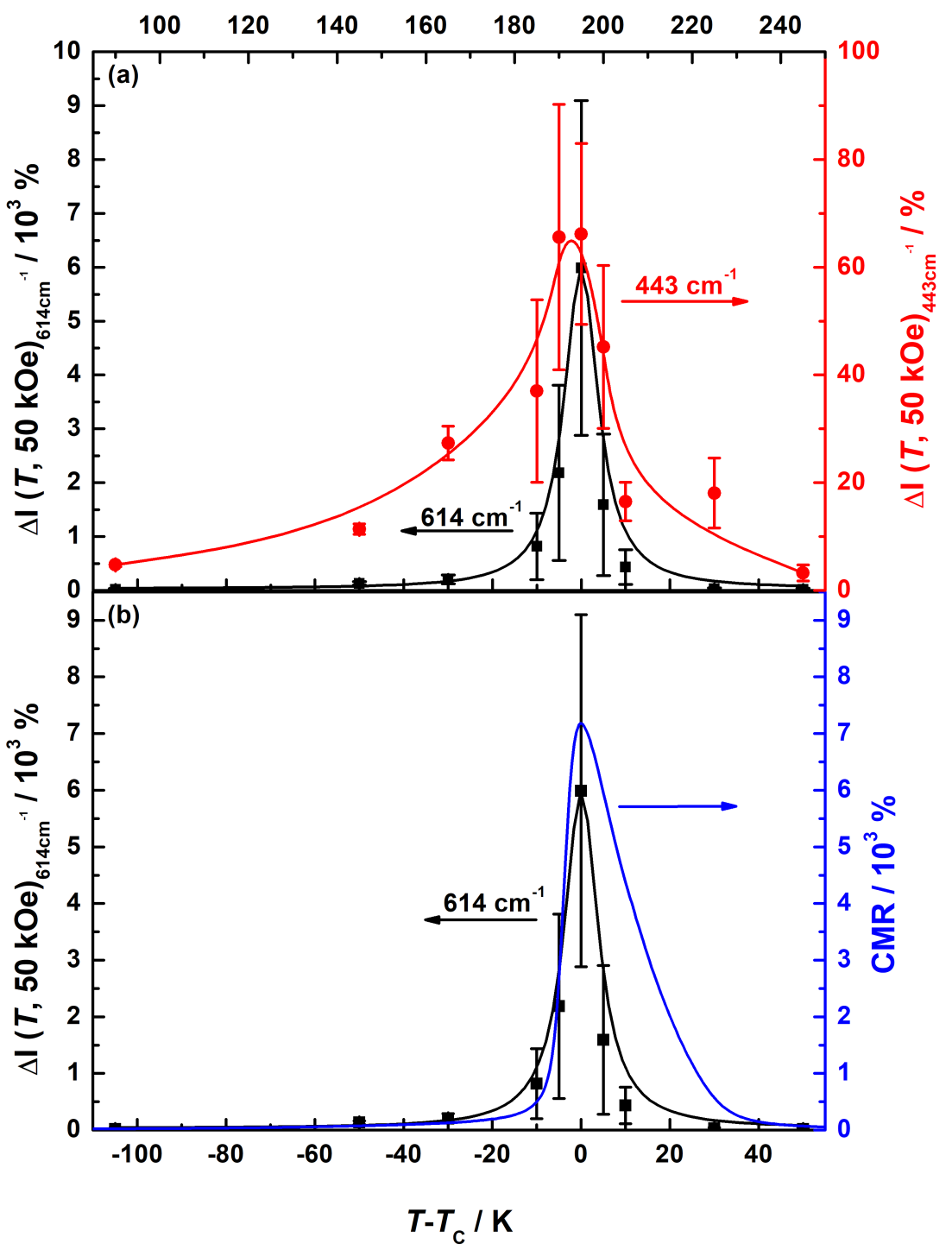

Figure 4.3.: (a) The $\Delta I_{j}(T, 50 \mathrm{kOe})$ ratios of the JT mode $\left(\omega_{\mathrm{s}}\right.$, left scale) and the bending mode ( $\omega_{\text {bend }}$, right scale) differing about two orders of magnitude $\left(\Delta I_{\mathrm{s}}(T, 50 \mathrm{kOe}) \approx 6000 \%, \Delta I_{\text {bend }}(T, 50 \mathrm{kOe})(\mathrm{T}, 50 \mathrm{kOe}) \approx 65 \%\right)$. For both modes, $\Delta I$ develops below $T=225 \mathrm{~K}$ coinciding with the metamagnetic transition temperature. (b) A comparison between the $\Delta I_{\mathrm{s}}$ (left scale) and the CMR ratio (right scale) illustrates their common polaronic nature.

scale, but $\Delta I_{\mathrm{s}}(T, 50 \mathrm{kOe})$ was taken locally on the $\mu \mathrm{m}$-scale. In addition, the probed systems are different, i.e. electric in case of CMR and phononic in $\Delta I_{\mathrm{s}}(T, 50 \mathrm{kOe})$. Nevertheless, the comparable $\Delta I_{\mathrm{s}}(T, 50 \mathrm{kOe})$ and CMR ratios demonstrate unambiguously the importance of the correlated JT polarons, giving evidence that their reduction is the main mechanism underlying the CMR effect. 

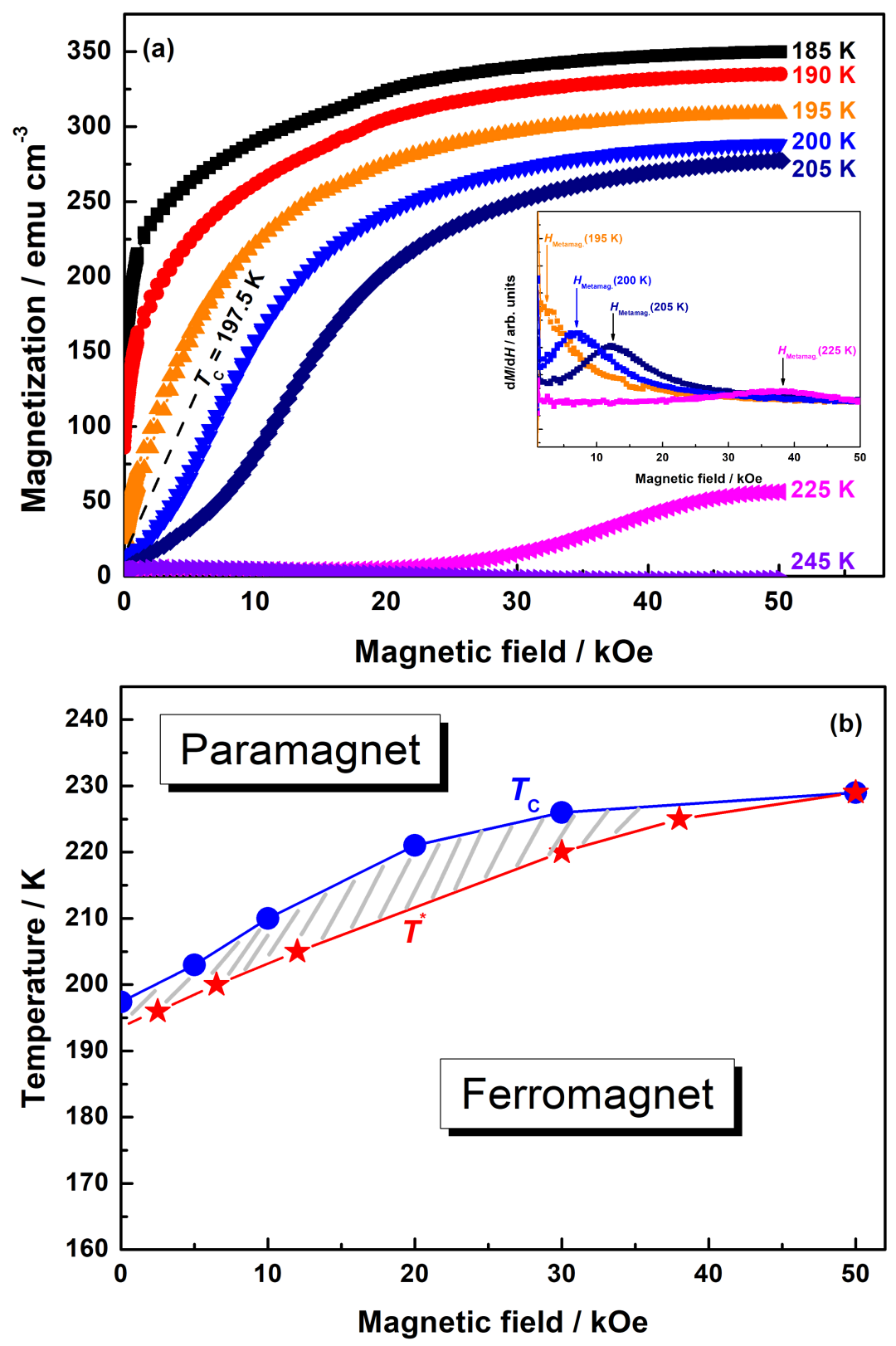

Figure 4.4.: (a) Field dependencies of the magnetization measured in the vicinity of $T_{\mathrm{C}}$, measured for magnetic fields $H=0-50 \mathrm{kOe}$. The inset shows $\mathrm{d} M / \mathrm{d} H$ curves for selected temperatures, $T=195 \mathrm{~K}, 200 \mathrm{~K}, 205 \mathrm{~K}$, $225 \mathrm{~K}$. (b) The evaluated $T$ - $H$ phase diagram with $T_{\mathrm{C}}$ and $T^{*}$ lines illustrates the existence of an inhomogeneous magnetic state for $T^{*}<T$ $<T_{\mathrm{C}}$, where FM nanodomains are AFM coupled by correlated JT polarons. Their field-induced melting results in a metamagnetic transition, accompanied by $\Delta I_{j}(T, 50 \mathrm{kOe})$ and CMR behavior. 
The origin of the magnetic-field-dependent Raman behavior and its relation to the magnetic phase transition and the CMR can be illustrated by a $T-H$ phase diagram, obtained by measuring the magnetization loops $M(H)$ at different temperatures across the phase transition, shown in Fig.4.4 (see also supplementary information S4 in appendix A). The obtained $T$ - $H$ phase diagram, shown in Fig $4.4 \mathrm{p}$, reveals two important temperature scales: 1$)$ the $T_{\mathrm{C}^{-}}(\mathrm{H})$-line indicating a magnetic field dependence with a saturating value of $T_{\mathrm{C}}(H=50 \mathrm{kOe})=229 \mathrm{~K}$ and 2$)$ the $T^{*}(H)$ line separating a mixed state with a metamagnetic transition from a homogeneous FM state, which is developed for $T<T^{*}(H)$. The narrow temperature distribution of the $\Delta I_{\mathrm{s}}(T, 50 \mathrm{kOe}), \Delta T_{\Delta I(T, 50 \mathrm{kOe})}= \pm 10 \mathrm{~K}$, and the $\mathrm{CMR}, \Delta T_{\mathrm{CMR}}= \pm 30 \mathrm{~K}$, around $T_{\mathrm{C}}$ shows their common origin and location between the $T^{*}$ - and $T_{\mathrm{C}}$-lines in the $T$ - $H$ diagram, i.e. within the inhomogeneous magnetic state. Such a complex magnetic behavior close to the first order phase transition was introduced in earlier theoretical CMR studies [1, 164] and supported by magnetic [66, 152] and ultrasonic [150] experiments on LPCMO. An applied magnetic field couples to the short-rangeordered polaronic AFM phase and melts the polaronic phase by aligning the spins parallel to the field, yielding the suppression of the JT modes in the Raman spectra. These changes go along with a drastic increase of the magnetic moment, i.e. a metamagnetic transition, and a reduction of the resistance manifesting as the CMR. Note that, both temperatures, $T_{\mathrm{C}}$ and $T^{*}$, merge into a common value in the $T$ - $H$ phase diagram, which is related to the temperature of the charge ordering $(\mathrm{CO})$ transition, $T_{\mathrm{CO}} \approx 225-230 \mathrm{~K}$, in manganites [152]. Above $T_{\mathrm{CO}}$, the PM phase remains fully symmetric, since neither CO and nor AFM correlations are energetically more favorable.

As mentioned before, the doping of the parent compound $\mathrm{LaMnO}_{3}$ with divalent cations induces an oxygen disorder in the system and thus, in the $\mathrm{MnO}_{6}$ network, which results in a broadening of the JT modes [65. Lowering the temperature below $T_{\mathrm{C}}$, suppresses the polaronic phase, i.e. the disorder in the system, resulting in an increase of structural order within the $\mathrm{MnO}_{6}$ network, reflected by the intensity increase of the bending mode $\omega_{\text {bend }}$. A similar process occurs in an applied magnetic field in the vicinity of $T_{\mathrm{C}}$. The suppression of the polaronic phase results in a strong suppression of the JT mode $\left(\omega_{\mathrm{s}}\right)$ and in a more moderate increase of the intensity of $\omega_{\text {bend. }}$. This means that the field-induced decrease of disorder close to $T_{\mathrm{C}}$ does not seem to be instantly converted into the same amount of structural order in the $\mathrm{MnO}_{6}$ system. Since the octahedral system remains at a relatively high temperature $\left(T=T_{\mathrm{C}} \approx 197 \mathrm{~K}\right)$, the disorder-order conversion is relatively ineffective. The reason is, likely, the lifetime $\tau_{h}$ of a $\mathrm{Mn}^{3+}$ state and its strongly non-linear temperature behavior close to $T_{\mathrm{C}}$ [149]. At low temperatures, i.e. $\tau_{h} \ll \tau_{J T}$, the further decrease of $\tau_{h}$ in an applied magnetic field enables an instantaneous gain in order. Close to the phase transition, $\tau_{h}$ is comparable to $\tau_{J T}$ and only a small amount of disorder can be transformed. Since $\tau_{h}<\tau_{J T}$, the JT distortion, however, can be significantly reduced resulting in a strong magnetic-field-induced effect for $\omega_{\mathrm{s}}$, but a much smaller for the bending mode $\omega_{\text {bend }}$. Temperature-dependent measurements at 

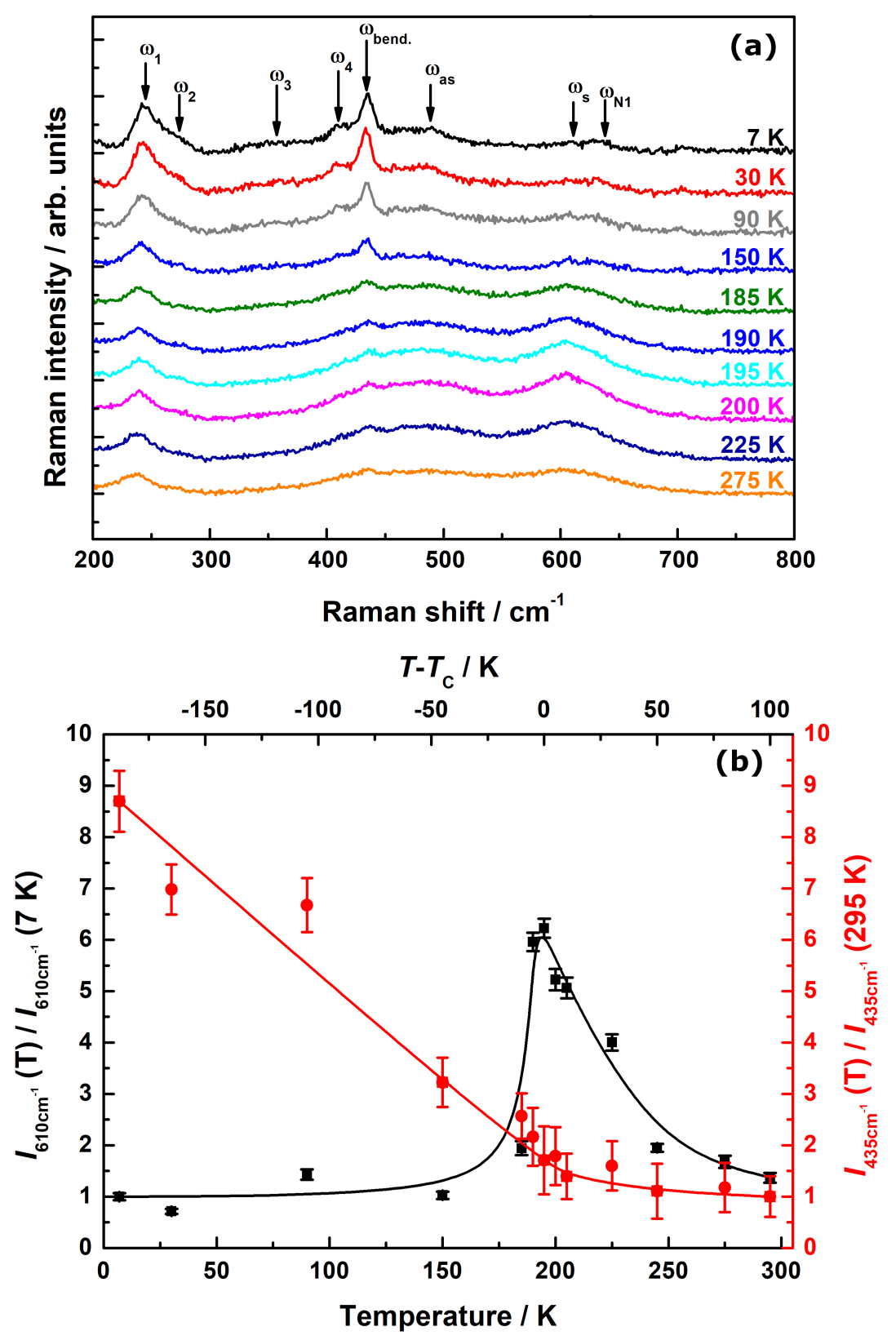

Figure 4.5.: Temperature-dependent Raman spectra of LPCMO (a) and the evaluated temperature dependency (b) of the intensity of the JT mode $\omega_{\mathrm{s}}\left(610 \mathrm{~cm}^{-1}\right.$, black $)$ and of the bending mode $\omega_{\text {bend }}\left(435 \mathrm{~cm}^{-1}\right.$, red $)$.

ambient magnetic field, $H=0 \mathrm{kOe}$, show a similar behavior (see Fig 4.5). Below $T_{\mathrm{C}}$, the polaronic phase shrinks rapidly and remains at a constant level, represented by the small intensity of the JT mode $\omega_{\mathrm{s}}$. The bending mode, in turn, gains significant intensity only below $T_{\mathrm{C}}$, continuously increasing down to $7 \mathrm{~K}$. One could expect a further field-induced increase of the bending mode at $T=T_{\mathrm{C}}$, i.e. higher values of $\Delta I_{\text {bend }}(T, 50 \mathrm{kOe})$ for $H>50 \mathrm{kOe}$ analogously to its temperature dependence. 
In summary, magnetic-field-dependent Raman spectroscopy on a thin LPCMO film in a broad range of temperatures and magnetic fields was carried out. We obtained direct evidence that the reduction of the amount of correlated JT polarons is the main mechanism underlying the CMR effect emphasizing the importance of strong electron-phonon coupling for CMR materials.

\subsection{Methods}

LPCMO film has been grown by a metalorganic aerosol deposition (MAD) technique [98] on commercial $\mathrm{MgO}(100)$ substrate (Crystal GmbH). Acetylacetonates of La, $\mathrm{Pr}$, Ca and $\mathrm{Mn}$ were used as precursors. The films was deposited at a substrate temperature $T_{\text {sub }}=950^{\circ} \mathrm{C}$ with a growth rate $v=10 \mathrm{~nm} / \mathrm{min}$ and was cooled down to room temperature in $30 \mathrm{~min}$ after deposition. X-ray diffraction in $\Theta-2 \Theta$ BraggBrentano geometry with $\mathrm{Cu}-\mathrm{k}_{\alpha}$ radiation and small-angle $\mathrm{x}$-ray reflectivity were performed to characterize the structure and thickness of the films. Magnetic (SQUID MPMS, Quantum design) and electrical four-probe characterizations (PPMS, Quantum design) were carried out for temperatures $T=5-300 \mathrm{~K}$ and magnetic fields $H=0-50 \mathrm{kOe}$. Raman measurements were performed in a backscattering-geometry with a continuous-wave Nd:YAG laser $(\lambda=532 \mathrm{~nm})$ by means of a confocal Raman microscope (LabRAM HR Evolution, Horiba Jobin Yvon), equipped with a thermoelectrically cooled charge-coupled device of $1024 \times 256$ pixels. The laser beam was focused onto the sample surface within a spot size, $d_{\text {Spot }} \approx 1.29 \mu \mathrm{m}$. To avoid significant heating of the sample, the laser power was kept at $P=2.9 \mathrm{~mW}$ (see supplementary information $\mathrm{S} 2$ in appendix A). The Raman setup was optically coupled to a continuous-flow He cryostat (Microstat MO, Oxford Instruments) to measure Raman spectra for temperatures, $T=90-245 \mathrm{~K}$, and magnetic fields, $H=0-50 \mathrm{kOe}$. A superconducting solenoid produces a magnetic field perpendicular to the sample surface (see supplementary information S3 in appendix A). No correction of the spectrometer response was made.

\subsection{Acknowledgement}

This work was financially supported financially by the A02, B04 and B01 project of the Sonderforschungsbereich SFB 1073 by the Deutsche Forschungsgemeinschaft DFG. 


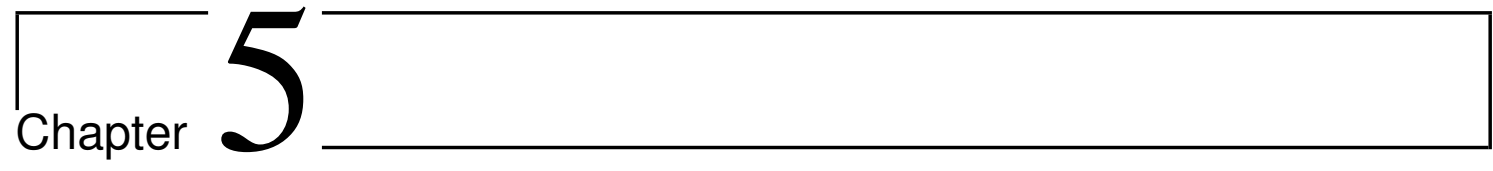

\section{Publication 3}

Metalorganic-aerosol-deposited Au nanoparticles for the characterization of ultrathin films by Surface-Enhanced Raman Spectroscopy

S. Merten ${ }^{1}$, V. Roddatis ${ }^{2}$ and V. Moshnyaga ${ }^{1}$

${ }^{1}$ I. Physikalisches Institut, Georg-August University Göttingen, Friedrich-Hund-Platz 1, D-37077, Göttingen

${ }^{2}$ Institut für Materialphysik, Georg-August University Göttingen, Friedrich-Hund-Platz 1, D-37077, Göttingen

Applied Physics Letters 115, 151902 (2019)

DOI: $10.1063 / 1.5121601$

Accepted for publication on September $15^{\text {th }}, 2019$

Published online October $8^{\text {th }}, 2019$

Reproduced from Applied Physics Letters 115, 151902 (2019) with the permission of AIP Publishing. 


\subsection{Motivation and Summary}

Surface-enhanced Raman spectroscopy is rarely used for the surface studies of thin oxide films. Mainly, this is caused by the challenge of a noninvasive deposition of the metallic nanostructures required for SERS. In particular, deoxygenation effects alter the surface structure, thus, potentially influencing the experimental results. These effects are further enhanced by the deposition of a metallic layer on top of the oxide. Therefore, a first step to facilitate SERS studies on correlated oxides would be to develop a method to prevent, or at least to minimize, those effects.

In the following chapter a new approach for ultrathin film characterization by means of surface-enhanced Raman spectroscopy was realized and applied to nanocrystalline $\mathrm{TiO}_{2}$ films. The results presented in the following chapter are summarized in Ref.[360]. The vacuum-free metalorganic aerosol deposition technique was used to grow gold nanoparticles on top of the surface of thin $\mathrm{TiO}_{2}$ films. An averaged enhancement factor of $10^{7}$ as well as the characterization of a $3 \mathrm{~nm}$ thin film was accomplished. Since the thin film acts as a substrate itself, this deposition technique can, therefore, be used for the characterization of a variety of solid thin film materials by means of SERS. Furthermore, MAD-based nanoparticle growth can be of special interest for oxide films due to the high partial pressure of oxygen and the constant oxygen flow during the deposition.

\subsection{Introduction}

Functional oxides play an important role in contemporary and future electronic devices, e.g. as high- $\kappa$ gate dielectrics [361] or as magnetoresistive devices and spin-valve heterostructures in spintronics [362, 363]. Especially, ultrathin films or oxide interfaces show novel functionalities, which are promising for new electronic devices [364]. Commonly used techniques to characterize structural properties of solid thin films are x-ray diffraction (XRD) and transmission electron microscopy (TEM). However, both techniques are difficult to employ for ultrathin films of only a few unit cells $(d<5 \mathrm{~nm})$, making the structural characterization of such films quite challenging. The small scattering volume, disorder or an improper crystallization yields a broadening of the diffraction peaks and may result in a vanishing signal [365].

In the last decades, Raman spectroscopy has been proven to be a versatile and noninvasive tool to characterize microstructures, nanoscale materials $(d>6 \mathrm{~nm})$ and even amorphous samples [49 51]. Unfortunately, the low scattering efficiency of the Raman process still hampers the practical application for the characterization of ultrathin films. Its poor sensitivity, however, can be overcome by using Surface-Enhanced Raman Spectroscopy (SERS) [68]. This technique exploits the locally enhanced electromagnetic field near the surface of metallic nanostructures due to the excitation of localized surface plasmon resonances (LSPR). But, until 
now, only a few attempts were made to facilitate SERS for the characterization of thin solid films [82, 83]. A critical issue is to ensure a noninvasive growth of the required metallic nanostructures near or on the studied thin film system, making its application for thin film characterization quite challenging. Furthermore, the growth of thin films directly on the metallic nanostructure can be excluded, as the required high roughness of the nanostructures prevents an epitaxial growth of the film. Therefore, the ideal way to establish SERS for thin film characterization would be the growth of the metallic nanostructure directly onto the surface of the studied thin film. However, most of standard physical vapor deposition techniques, such as pulsed-laser deposition, thermal evaporation or sputtering, require ultrahigh vacuum and elevated temperatures. These conditions often lead to an oxygen deficiency in oxide films [87, 88] and, thus, altering the structural properties of the material. Such an oxygen loss can be further enhanced by the deposition of a metal layer, like $\mathrm{Ag}, \mathrm{Au}, \mathrm{Pt}$ or $\mathrm{Cu}$, onto the surface [94, 97]. A promising chemical route to prevent deoxygenation effects could be provided by the metalorganic aerosol deposition (MAD) [98, which has proven to be a powerful technique to grow oxide films [99] as well as complex oxide heterostructures [100, 101. The continuous oxygen supply and the associated high oxygen partial pressure up to 1 bar during the deposition could be of great advantage, thus, making the MAD a promising technique to enable SERS studies on thin oxide films.

In this letter, we demonstrate the possibility of growing $\mathrm{Au}$ nanoparticles by the vacuum-free MAD technique and performed the structural characterization of thin films of the well-known oxide system $\mathrm{TiO}_{2}$ by means of SERS. We observed a significant enhancement of the $E_{\mathrm{g}}$ mode of $\mathrm{TiO}_{2}$ by a factor of $10^{7}$ in the $\mathrm{Au} / \mathrm{TiO}_{2}$ structure and were able to accomplish the characterization of a 3-4 nm thin $\mathrm{TiO}_{2}$ film.

\subsection{Experimentel Techniques}

Thin $\mathrm{TiO}_{2}$ films and $\mathrm{Au}$ nanoparticles were prepared by MAD [98] by using commercially available precursors titanium(IV) diisopropoxidebis and gold(III)acetate, which were dissolved in dimethylformamide to a concentration of $0.02 \mathrm{~mol} / \mathrm{l}$. Subsequently, $\mathrm{TiO}_{2}$ films of different thicknesses, namely $d=3,7,14,60 \mathrm{~nm}$, were grown at $960^{\circ} \mathrm{C}$ on $\mathrm{Si}(100)$ substrates (Crystal $\mathrm{GmbH}$ ). Finally, $1 \mathrm{ml}$ of the Au precursor solution was sprayed onto the $\mathrm{TiO}_{2}$ film surface at a deposition temperature, $T_{\mathrm{Au}}=550-600^{\circ} \mathrm{C}$, with a corresponding growth rate of $0.11 \mathrm{ml} \mathrm{s}^{-1}$. Structural analysis of the $\mathrm{TiO}_{2}$ films was carried out by using BRUKER D8 diffractometer by x-ray diffraction (XRD) using $\mathrm{Cu}_{k \alpha}$ radiation. Transmission electron microscopy (TEM) studies were performed by using a Thermofisher Titan 80-300 operated by $300 \mathrm{kV}$. Cross-sections were prepared by mechanical polishing followed by $\mathrm{Ar}^{+}$ion milling using a Gatan Precision Ion Polishing System (PIPS 695). The surface morphology of the grown Au-layer was examined by scanning electron microscopy (SEM, Leo 
Supra 35) and the LSPR wavelength was obtained by a four-lens optical reflectivity setup under normal incidence. Raman spectra were acquired by a JobinYvon Horiba LabRAM HR Evolution spectrometer equipped with a continuous-wave HeNe-laser at $632.8 \mathrm{~nm}$ and a thermoelectrically cooled charge-coupled device of $1024 \times 256$ pixels and a depth resolution, $\delta<2 \mu \mathrm{m}$. The spot size of the incident laser beam was $\approx 1 \mu \mathrm{m}$ and the laser power was kept below $1 \mathrm{~mW}$ to avoid heating.

\subsection{Results \& Discussion}

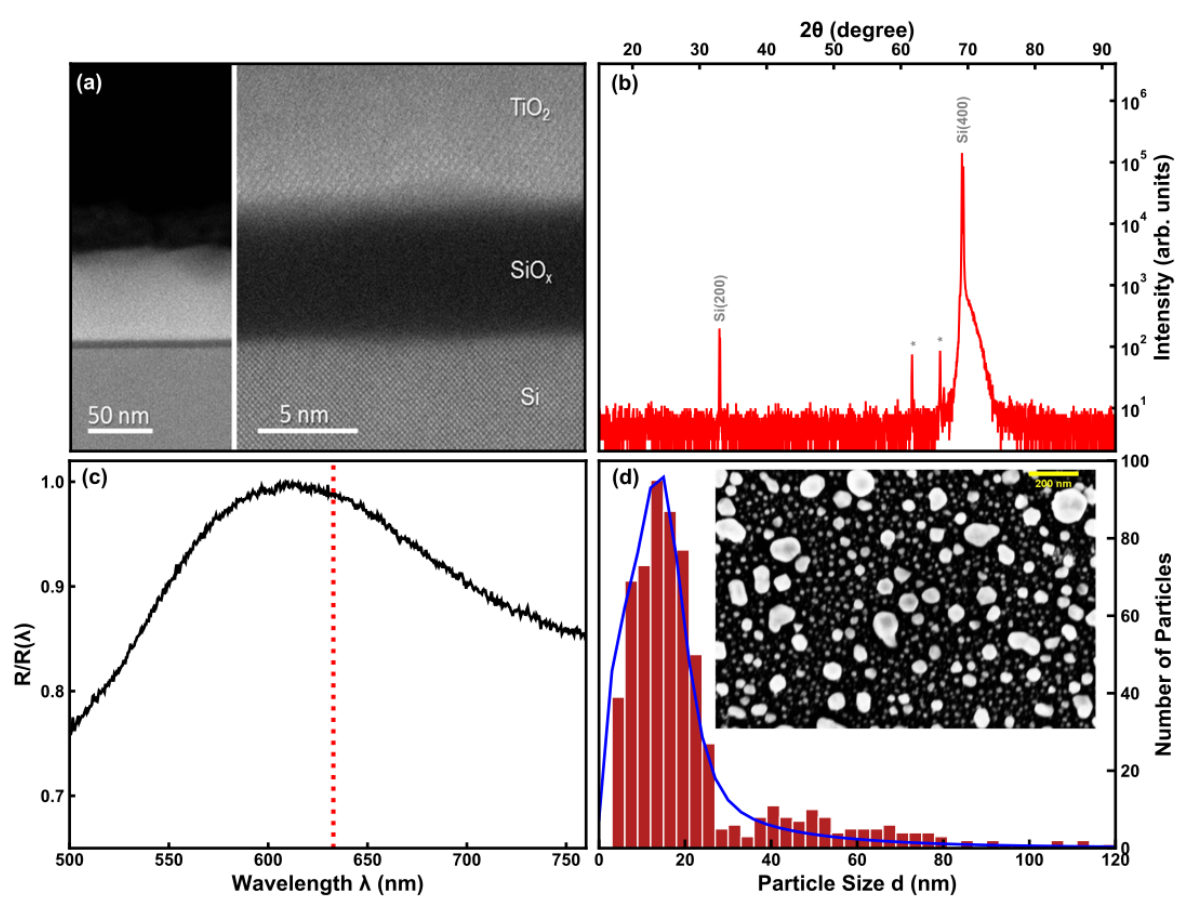

Figure 5.1.: TEM images (a) show the nanoscale crystalline growth of $\mathrm{TiO}_{2}$ on $\mathrm{Si}(100)$, which is supported by the missing $\mathrm{TiO}_{2}$ reflections in the XRD pattern (b). Naturally, a thin buffer layer of $\mathrm{SiO}_{2}$ is formed due to the ambient pressure during deposition. Optical reflectivity measurements (c) show a LSPR wavelength, $\lambda=613 \mathrm{~nm}$, which lies near the used excitation line (dotted red line). SEM pictures of an $1.5 \times 1.1 \mu \mathrm{m}^{2}$ area show the formation of homogeneously distributed $\mathrm{Au}-\mathrm{NPs}$ with a mean particle size, $d_{\mathrm{Au}} \approx 16 \mathrm{~nm}$, and an average surface coverage of $58 \mu \mathrm{m}^{-2}$ of the enhancing particle $(d)$.

The structural characterization by XRD and TEM was carried out on a $60 \mathrm{~nm}$ thick film to guarantee a large enough scattering volume for the characterization. As we were interested in the characterization of (ultra) thin films, the further study by Raman spectroscopy as well as the Au deposition was performed on films with thicknesses, $d=3-15 \mathrm{~nm}$. The correspondent deposition volumes were deduced from 
the volume used for the $60 \mathrm{~nm}$ thick film. TEM images, shown in Fig.5.17, reveal the formation of nanoscale regions, but, no complete crystallization through the whole $60 \mathrm{~nm}$ thick oxide film. This is supported by the XRD pattern (see Fig.5.1p), which shows no indication of any of the common $\mathrm{TiO}_{2}$ phases, namely anatase and rutile. The sharp peak around $32.96^{\circ}$ can be assigned to the basis-forbidden $\mathrm{Si}(200)$ reflection, which is often observed in XRD patterns of $\operatorname{Si}(100)$ substrates [366, 367] and can be explained by so-called Umweganregungen [368]. Furthermore, a $5 \mathrm{~nm}$ thick buffer layer at the film/substrate interface is clearly visible, which is, most likely, $\mathrm{SiO}_{2}$. Since the films were deposited at ambient atmosphere and elevated temperatures, a naturally grown amorphous $\mathrm{SiO}_{2}$ buffer layer at the $\mathrm{Si}(100)$ surface cannot be prevented and, probably, causing a nanoscale crystalline growth of $\mathrm{TiO}_{2}$. Optical measurements of the Au-covered film show a LSPR wavelength, $\lambda_{\mathrm{LSPR}}=613 \mathrm{~nm}$, which lies near the used excitation, $\lambda_{\text {inc }}=632.8 \mathrm{~nm}$ (see Fig 5.1k). SEM pictures, shown in Fig.5.1d, reveal the formation of homogeneously distributed Au-NPs. The particle size is, however, distributed over a rather broad range with a characteristic tail to larger diameters, commonly seen for aerosol- or vapor-based nanoparticle growth [369, 370]. A mean particle size, $d_{\mathrm{Au}} \approx 16 \mathrm{~nm}$, with a surface coverage of $58 \mu \mathrm{m}^{-2}$ was obtained by means of the particle size distribution.

Figure 5.2 shows the acquired Raman spectra of a $14 \mathrm{~nm}$ thick bare $\mathrm{TiO}_{2}$ film as well as of the same sample covered by Au-NPs at room temperature. Without the $\mathrm{Au}-\mathrm{NPs}$, only a tiny Raman signal around $142 \mathrm{~cm}^{-1}$ was detected, which corresponds well to the $E_{\mathrm{g}}(1)$ phonon mode of the anatase phase of $\mathrm{TiO}_{2}$ [371] (see the insert in Fig 5.2 ). The weak signal will probably be caused by the relatively low Raman scattering efficiency of the $14 \mathrm{~nm}$ film as well as the nanoscale crystalline growth. Since Raman spectroscopy is sensitive to the crystallinity and microstructure of a material, Raman lines become weak and broad when the lattice is imperfect [51]. Therefore, the nanocrystalline structure of our $\mathrm{TiO}_{2}$ films suppresses the phonon modes of $\mathrm{TiO}_{2}$ resulting in the almost vanishing Raman signal. In contrast, the intensity of the $E_{\mathrm{g}}(1)$ mode is significantly enhanced by a factor of $\approx 200$ for the film covered by Au-NPs. Furthermore, an additional mode at $635 \mathrm{~cm}^{-1}$ is observed, which is also associated with the anatase phase $\left(E_{\mathrm{g}}(2)\right)$ 371]. However, no signs of the rutile phase [371] with its strongest Raman-active mode at $612 \mathrm{~cm}^{-1}$ or the amorphous combination band at $675 \mathrm{~cm}^{-1}$ seen in $\mathrm{TiO}_{2} /$ Klarite films [82] can be detected. To determine whether the intensity enhancement is homogeneously distributed over the sample and to exclude the occurrence of "enhancement voids", i.e. points without an intensity enhancement, we performed a Raman mapping of a $48 \times 48 \mu \mathrm{m}^{2}$ area with a step size of $6 \mu \mathrm{m}$. As one can see in Fig, $5.2 \mathrm{~b}$, the Raman mapping does not show any enhancement voids, but an almost homogeneous enhancement by at least a factor of 250 over the whole mapping area. The variations of the signal enhancement can be, most likely, attributed to an inhomogeneous clustering of the enhancing particles, i.e. a higher amount of Au-NPs at a given point and thus, a higher concentration of probed unit cells. Such clustering can result in a signal enhancement by a factor of 2000 at certain points; one order of magnitude 
higher than the average enhancement of the SERS-active substrate.

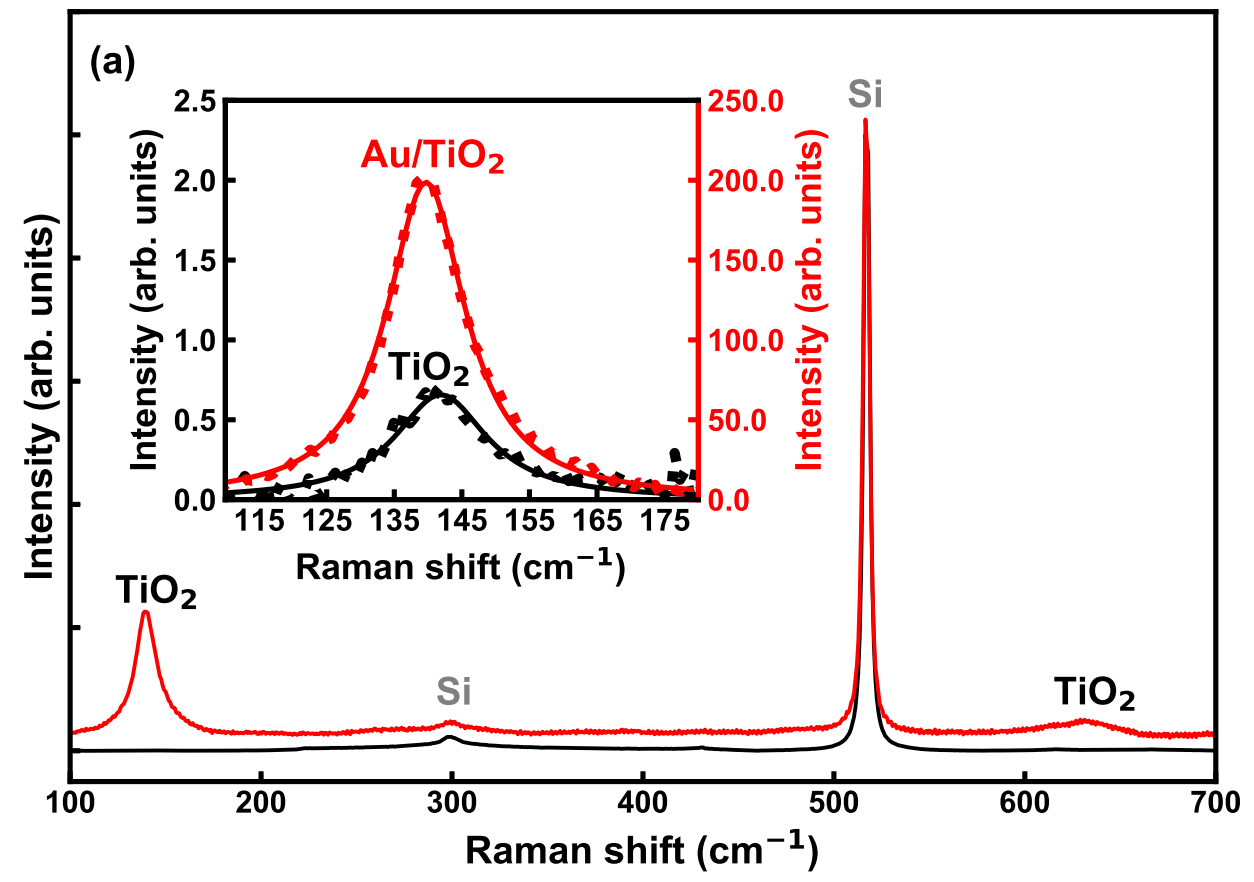

(b)

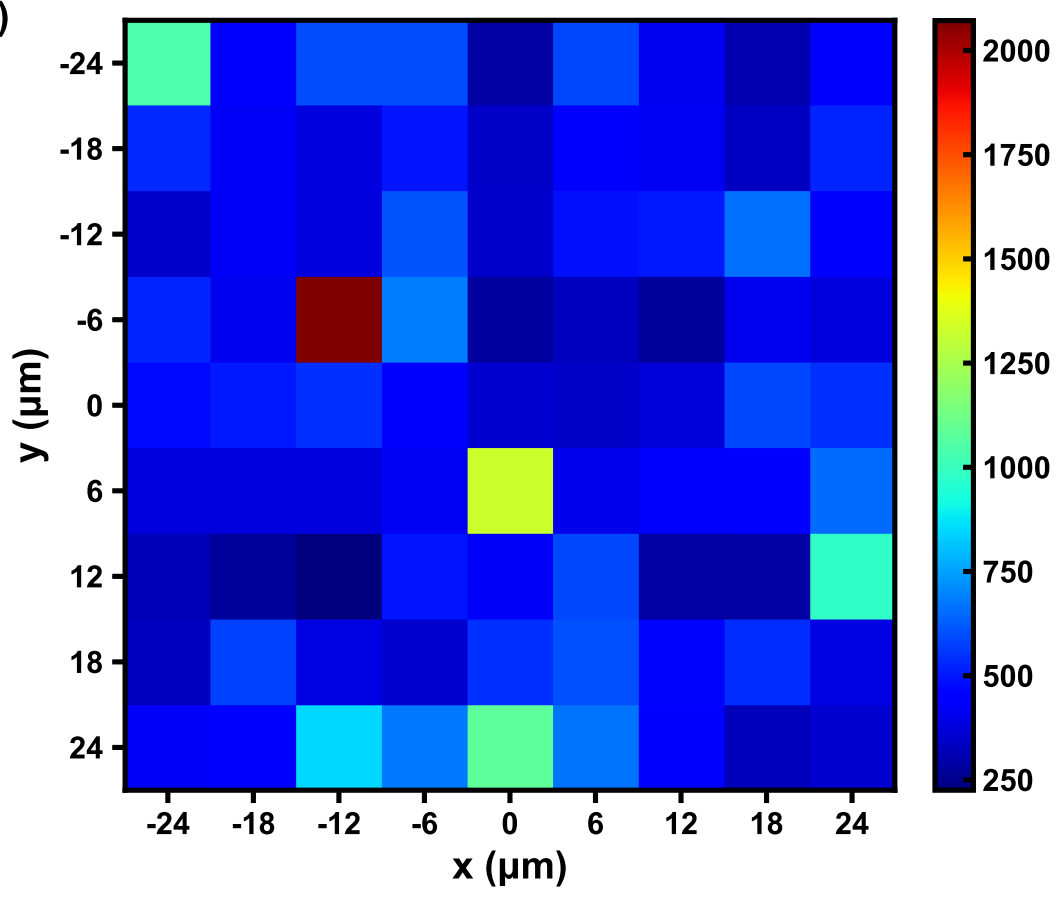

Figure 5.2.: (a) Comparison of the conventional Raman signal of the $14 \mathrm{~nm}$ thick $\mathrm{TiO}_{2}$ film (black) and the SER signal obtained by the same $\mathrm{TiO}_{2}$ film covered by Au-NPs (red). The Raman Mapping (b) of a $48 \times 48 \mu \mathrm{m}^{2}$ area shows a homogeneous enhancement of the Raman signal. 
The intensity ratio, however, neglects the concentration of the probed analyte. Therefore, one typically normalizes the intensity enhancement to the number of probed analyte in the SER and the conventional Raman experiment, leading to the most common definition of the SERS enhancement factor $(E F)$ of a given SERSsubstrate [78]

$$
E F=\frac{I_{S E R S} N_{R S}}{I_{R S} N_{S E R S}}
$$

where $N_{\mathrm{RS}}$ and $N_{\mathrm{SERS}}$ represent the number of molecules/unit cells adsorbed on the SERS-substrate within the laser spot area and the number of molecules/unit cells probed by conventional Raman spectroscopy, respectively. $I_{\text {SERS }}$ correspond to the SER intensity and $I_{\mathrm{RS}}$ to the conventional Raman scattering intensity of a given vibrational mode. However, the magnitude of the $E F$ depends strongly on the determination of $N_{i}$ and the estimated $E F$ tends to be overestimated [78]. A comprehensive and critical review of the different definitions of the $E F$ was made by LE RU and co-workers [78]. They proposed a more rigorous definition of the $E F$ in Eq. (5.1), defining it as

$$
E F=\frac{I_{S E R S} H_{e f f} c_{R S}}{I_{R S} \mu_{S} \mu_{M} A_{M}}
$$

where $\mu_{\mathrm{S}}$ is the surface density of the analyte on a single metal particle, $\mu_{\mathrm{M}}$ the surface density of the individual nanoparticles enhancing the Raman signal, i.e. the obtained surface coverage, $A_{\mathrm{M}}$ the surface area of an individual metallic particle, $H_{\text {eff }}$ the effective height of the scattering volume [78] and $c_{\mathrm{RS}}$ the concentration of the analyte in the conventional Raman scattering experiment. For the estimation of the $E F$, we used the intensity of the $E_{\mathrm{g}}$ mode at $143 \mathrm{~cm}^{-1}$ and followed the remarks given in Ref.[78]. The concentration $c_{\mathrm{RS}}$ is represented by the number of unit cells $N$ (u.c.) in the scattering volume $V$ yielding

$$
c_{\mathrm{RS}}=\frac{N(\text { u.c. })}{V}=\frac{V / V(\text { u.c. })}{V}=\frac{1}{a^{2} c}
$$

where $a$ and $c$ are the lattice constants of $\mathrm{TiO}_{2}$. For the calculation of the $E F$, we used the bulk lattice constants of anatase $\mathrm{TiO}_{2}[372, a=3.784 \AA$ and $c=9.512 \AA$, respectively, and calculated an effective scattering height, $H_{\text {eff }}=9.03 \mu \mathrm{m}$, by applying the definition given in Ref.[78]. The surface density of the analyte on the metal can be estimated by the number of unit cells within the area of the nanoparticle and, thus, yields $\mu_{\mathrm{S}}=\left(A_{\mathrm{NP}} a^{-2}\right) / A_{\mathrm{NP}}=1 / a^{2}$. Therefore, by using Eq. (5.2), we get an $E F \approx 10^{7}$, averaged over 81 spectra acquired at different positions on the $\mathrm{TiO}_{2}$ surface.

The estimated $E F$ is higher than those obtained for SERS substrates produced by metal vapor deposition [373] $\left(E F=10^{5}\right)$ and commercially available Klarite SERS 
substrates [374] $\left(E F=10^{6}\right)$. On the other hand, our MAD-based EF is comparable to those obtained for SERS substrates produced by a combination of a highly ordered, etched substrate and electron beam evaporation [375] $\left(E F=10^{7}\right)$. The latter one as well as the commercially available Klarite substrate, however, require a pretreatment of the substrate, e.g. etching or nanostructuring of the substrate to establish an ordered, monodisperse particle distribution, which produces the mentioned $E F$. The MAD-based SERS substrate prepared by a self-aggregated, polydisperse growth, however, achieves a comparable enhancement. Considering the resulting relatively broad particle size distribution of our SERS-active substrates and, thus, the inhomogeneous coverage of the enhancing particle, it is quite remarkable that a comparable $E F$ can be obtained by using this simple MAD-based approach. Note that, for the sake of the calculation, several assumptions were made, which affect the estimated EF. The particle was considered as an ideal spherical shape; the irregular, more elliptic shape, which can be seen in the SEM pictures, was neglected. For the calculation of the surface area of the metallic particle, we treated the particles as hemispheroid protrusions and thus, overestimated the surface area $A_{\mathrm{M}}$, since the cross section of the particle was taken into account and not the local curvature in contact with the substrate. Hence, the evaluated EF of our MAD-based SERS substrates will be underestimated by at least one order of magnitude. Furthermore, the electromagnetic enhancement might not be the sole mechanism contributing to the estimated $E F$. The enhancement of non-totally symmetric $E_{\mathrm{g}}$ modes can indicate a charge-transfer-induced resonance via Herzberg-Teller coupling [318, which could be mediated by the plasmonic excitation in the $\mathrm{Au}-\mathrm{TiO}_{2}$ system [82] However, it is generally accepted that the chemical SERS mechanism can only provide a $E F \leq 10^{2}$ [76], which is several orders of magnitudes lower than the one obtained for our MAD-based SERS substrates. Nonetheless, the calculated EF should provide a reasonable estimation of the enhancement, which can be obtained with our MAD-grown nanoparticles.

Finally, to obtain the detection limit of our SERS-active structures, we measured the SER spectra of $\mathrm{TiO}_{2}$ films with different thicknesses, $d=14 \mathrm{~nm}, 7 \mathrm{~nm}, \approx 3 \mathrm{~nm}$. As one can see in Fig.5.3a, we could not detect any Raman signal of the $3 \mathrm{~nm}$ thick film and just a tiny Raman signal of the $7 \mathrm{~nm}$ and $14 \mathrm{~nm}$ films was observed. In contrast, the SER spectra, seen in Fig.5.3b, have shown a clearly measurable Raman signal for all thicknesses. Note that the SER intensity slightly differs from sample to sample, which could be due to a small difference of the LSPR wavelength $\lambda_{\text {LSPR }}$ as well as due to the small scattering volume of the $3 \mathrm{~nm}$ film. Furthermore, a frequency shift by $1-2 \mathrm{~cm}^{-1}$ can be observed for the $7 \mathrm{~nm}$ and $14 \mathrm{~nm}$ thick films, while the shift in the ultrathin film can be neglected due to the overlapping statistical errors (see Tab.5.1). These small difference may indicate slight structural changes due to the annealing of the $\mathrm{TiO}_{2}$ film during the Au-NP deposition. A distinct narrowing of the linewidth in the conventional and SER spectra is detected, indicating an improved crystalline quality with elevated thickness. The smaller FWHM in the SER spectra might be explained by the different sensitivity of both Raman techniques. While the 


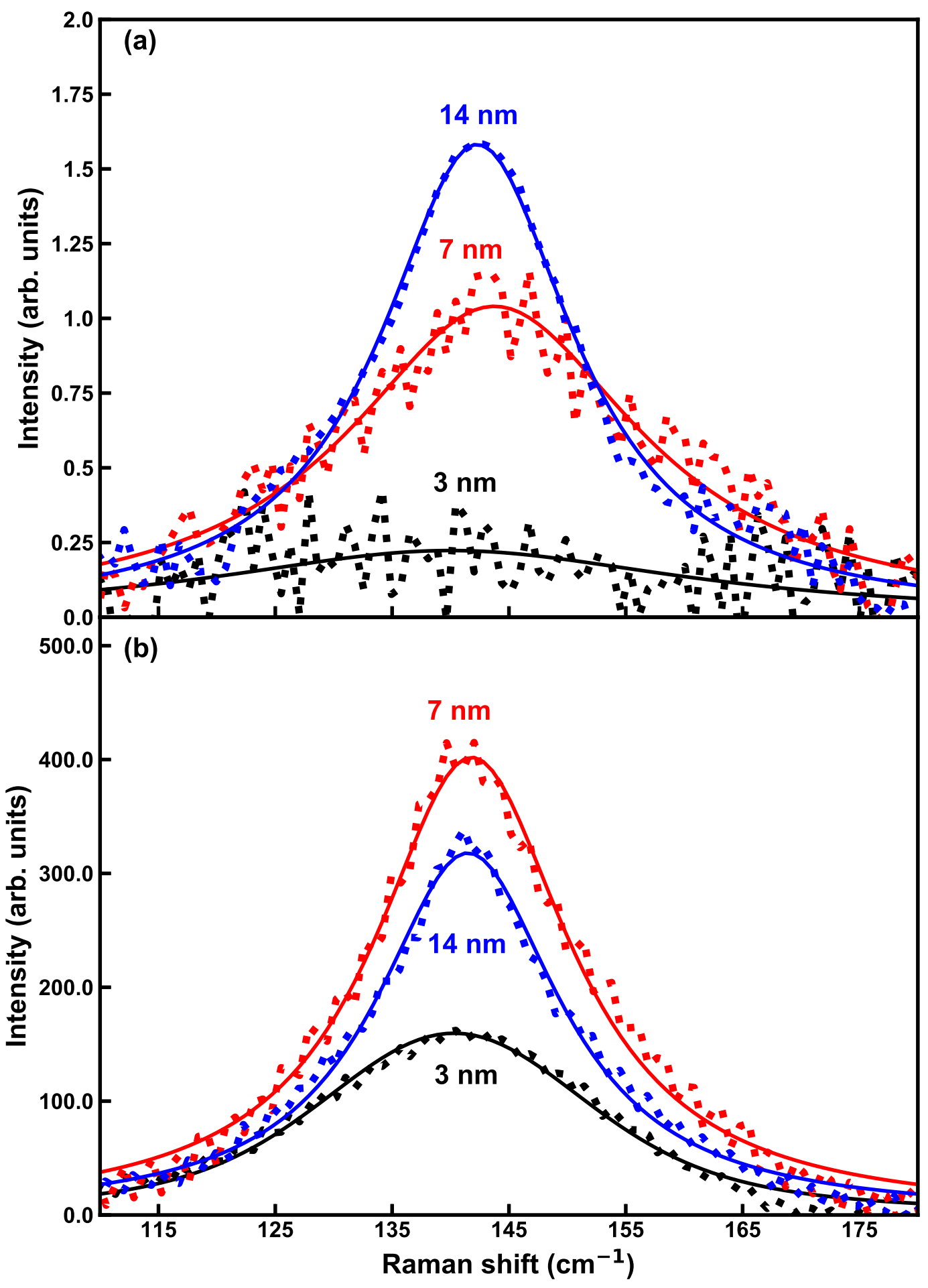

Figure 5.3.: Conventional Raman (a) and SER spectra (b) of thin $\mathrm{TiO}_{2}$ films with different thicknesses: $d \approx 3-4 \mathrm{~nm}$ (black), $d \approx 7 \mathrm{~nm}$ (red) and $d \approx 14 \mathrm{~nm}$ (blue). 
Table 5.1.: Linewidths and phonon frequencies in $\mathrm{cm}^{-1}$ of the $E_{\mathrm{g}}(1)$ in the conventional and SER spectra.

\begin{tabular}{c|c|c} 
& FWHM (conv. RS) & FWHM (SERS) \\
\hline $3 \mathrm{~nm}$ & $50.12 \pm 10.2$ & $27.96 \pm 0.52$ \\
$7 \mathrm{~nm}$ & $30.59 \pm 1.2$ & $20.42 \pm 0.41$ \\
$14 \mathrm{~nm}$ & $20.19 \pm 0.4$ & $19.08 \pm 0.30$ \\
& Raman shift (conv. RS) & Raman shift (SERS) \\
\hline $3 \mathrm{~nm}$ & $139.98 \pm 2.86$ & $140.31 \pm 0.20$ \\
$7 \mathrm{~nm}$ & $143.75 \pm 0.40$ & $141.80 \pm 0.15$ \\
$14 \mathrm{~nm}$ & $142.28 \pm 0.15$ & $141.49 \pm 0.10$
\end{tabular}

SER spectra will mainly probe the surface area, the conventional Raman spectra will also probe the highly amorphous interface region, which will be dominate due to the small thickness of the $\mathrm{TiO}_{2}$ films. By increasing the films's thickness or by enhancing the signal of the surface area, these contributions are suppressed due to the larger amount of probed nanoscale crystalline regions, resulting in a higher linewidth of the Raman peak. This is supported by the comparable FWHM of the $14 \mathrm{~nm}$ thick film in both kind of Raman spectra and the similar width of the $E_{\mathrm{g}}$ mode in the SER spectra of the $7 \mathrm{~nm}$ thick film. However, the estimated linewidth, $\approx 19-20 \mathrm{~cm}^{-1}$, is still three times larger than the one observed for single crystals $\left(7 \mathrm{~cm}^{-1}\right)[376]$. The linewidths and frequencies are summarized in Tab 5.1 .

Considering a (001) growth direction of the $\mathrm{TiO}_{2}$ film with a lattice constant 372, $c=9.512 \AA$, the observation of the Raman signal of a $3 \mathrm{~nm}$ thick film implies that we are able to structurally characterize ultrathin films consisting of only 3 u.c.. Note that the preferred enhancement of the $E_{\mathrm{g}}$ modes may also indicate the existence of $\{101\}$ facets at the surface, which results in a suppression of the $A_{\mathrm{g}}$ and $B_{1 \mathrm{~g}}$ modes [377]. Therefore, a favored enhancement of the non-totally symmetric modes may not be caused by the chemical SERS mechanism. Considering the amorphous nanocrystalline growth, an increased exposure of $\{101\}$ facets at the surface might further be possible, since it represents the thermodynamically most stable surface 378.

In summary, we introduced a new approach to produce SERS-active substrates for thin film characterization. A strong enhancement of the $E_{\mathrm{g}}$ modes of the anatase $\mathrm{TiO}_{2}$ phase was observed and an average enhancement factor of $\approx 10^{7}$ could be obtained. Moreover, the characterization of an 3 u.c. thin film could be easily accomplished showing the enormous capabilities of MAD-based SERS substrates for the characterization of ultrathin solid films. Since the film serves as the substrate itself, this technique can be easily applied to a variety of thin film materials. Furthermore, as the MAD technique provides a constant oxygen flow, this approach should be particularly suitable for oxides struggling with oxygen deficiencies and/or 
deoxygenation effects during the $\mathrm{Au}$ deposition. We believe that further improvement to a more ordered monodisperse particle size distribution, could push our MAD-based SERS substrates to the detection of single unit cells, thus, making the MAD a promising and, more important, a simple and reproducible technique for the fabrication of highly active SERS substrates.

\subsection{Acknowledgment}

Financial support from the Deutsche Forschungsgemeinschaft (DFG) via SFB 1073 (TP B04, B01, A02 and Z02) is acknowledged. 


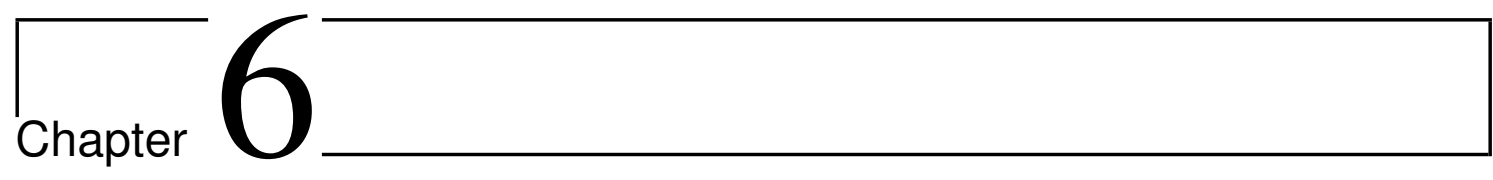

\section{Publication 4}

\section{Jahn-Teller reconstructed surface of the doped manganites shown by means of surface-enhanced Raman spectroscopy}

S. Merten ${ }^{1}$, V. Bruchmann-Bamberg ${ }^{1}$, B. Damaschke ${ }^{1}$, K. Samwer ${ }^{1}$ and V. Moshnyaga ${ }^{1}$

${ }^{1}$ I. Physikalisches Institut, Georg-August University Göttingen, Friedrich-Hund-Platz 1, D-37077, Göttingen

Physical Review Materials 3, 060401(R) (2019) as Rapid Communication

DOI: 10.1103/PhysRevMaterials.3.060401

Accepted for publication on April $30^{\text {th }}, 2019$

Published online June $28^{\text {th }}, 2019$

Licensed under a Creative Commons Attribution 4.0 International license (CC BY 4.0). 


\subsection{Motivation and Summary}

Microscopic and macroscopic interfaces are crucial for manganite physics. Domain boundaries of the electronically phase separated system can be considered as microscopic interfaces between the charge-ordered polaron-rich and the ferromagnetic metallic polaron-free regions. At the macroscopic interfaces, i.e. the surface and the film/substrate interface, electrical and magnetic dead layers are formed, which hinder the practical application of mixed-valence manganites in electronic devices (see Ch.2.1.3). Many of the recent theoretical and experimental results propose a charge redistribution at the surface/interface to accommodate the polar discontinuity [39, 41, 44, 206, 236, 238]. Such a charge redistribution would result in the enrichment of Jahn-Teller active $\mathrm{Mn}^{3+}$ ions at the surface/interface and, thus, in the localization of polarons. Therefore, a surface-sensitive technique such as surface-enhanced Raman spectroscopy should be able to determine whether such an enrichment of Jahn-Teller polarons occurs indeed. For this purpose, the in Ch.5presented approach was applied to the mixed-valence manganites $\mathrm{La}_{1-x} \mathrm{~A}_{x} \mathrm{MnO}_{3}$ and the results obtained were published in the article presented in this chapter. To the best of the author's knowledge, this publication constitutes the first SERS study on mixed-valence manganites.

Within this publication, direct evidence of the theoretically predicted electron-rich surface of doped perovskite manganites $\mathrm{La}_{0.7} \mathrm{~A}_{0.3} \mathrm{MnO}_{3}(\mathrm{~A}=\mathrm{Ca}, \mathrm{Sr})$ is presented by means of surface-enhanced Raman spectroscopy. The required Au nanoparticles were grown on top of thin manganite films by the metalorganic aerosol deposition technique (see $\mathrm{Ch} / 5$ and [360]), providing a stable oxygen atmosphere and prevents deoxygenation effects. The acquired surface-enhanced Raman spectra of rhombohedral $\mathrm{La}_{0.7} \mathrm{Sr}_{0.3} \mathrm{MnO}_{3}$ thin films reveal the symmetry-forbidden Jahn-Teller stretching modes demonstrating the predicted symmetry breaking at the surface and the fallback into the orthorhombic (Pnma) structure. These Jahn-Teller peaks arise from an electron accumulation at the surface and, hence, from the formation of $\mathrm{Mn}^{3+}$ and JT polarons.

\subsection{Introduction}

Mixed-valence perovskite manganites, like $\mathrm{La}_{1-x} \mathrm{~A}_{x} \mathrm{MnO}_{3}(\mathrm{~A}=\mathrm{Sr}, \mathrm{Ca})$, possess strong electron-spin-lattice correlations [1, 20], which result in many fundamentally interesting and useful electronic properties. Some of them, like a high degree of spin polarization [379] and a high catalytic activity [380, make manganites promising materials for many future applications, like tunnelling magnetoresistance (TMR) devices [381] or solid oxide fuel cells [382. Therefore, a detailed knowledge of the surface structure is of crucial importance, as the electronic surface structure determines the catalytic and fuel cell performance as well as the spin polarization at the TMR interface. 
Numerous theoretical studies have aimed at the understanding of the differently oriented $\mathrm{LaMnO}_{3}$ surfaces [200, 201], the (001) surface of $\mathrm{CaMnO}_{3}$ [203, 205] and the surface of the hole-doped $\mathrm{La}_{1-x} \mathrm{~A}_{x} \mathrm{MnO}_{3}(\mathrm{~A}=\mathrm{Ca}, \mathrm{Sr})$ [39, 40, 206, 383]. The alterations at the surface compared to the bulk structure range from large surface rumpling [40, 200 202, 206], over a spin-flip in the surface layer [201, 383] to a charge transfer from the bulk to the surface [39, 40, 206]. The latter results in the formation of a non-magnetic, most likely canted antiferromagnetic, insulating $\mathrm{Mn}^{3+}$-rich layer at the surface. Coaxial impact-collision ion scattering spectroscopy on $\mathrm{La}_{0.7} \mathrm{Sr}_{0.3} \mathrm{MnO}_{3}$ revealed a $\mathrm{MnO}_{2}$-terminated (001) surface [209]. Photoemission studies of $\mathrm{La}_{0.7} \mathrm{Ca}_{0.3} \mathrm{MnO}_{3}$ (LCMO) and $\mathrm{La}_{0.9} \mathrm{Ca}_{0.1} \mathrm{MnO}_{3}$ showed further that the surface termination depends also on the doping, changing from a $\mathrm{MnO}_{2}$ to a (La,Ca)O termination with decreasing $x$ [208]. A non-magnetic and insulating layer at the manganite surface was observed by conducting atomic force microscopy [187, 188] in $\mathrm{La}_{0.7} \mathrm{Ca}_{0.3} \mathrm{MnO}_{3}$ and by X-ray magnetic circular dichroism (XMCD) [93] as well as by X-ray resonant magnetic scattering (XRMS) [103, 384 on $\mathrm{La}_{0.7} \mathrm{Sr}_{0.3} \mathrm{MnO}_{3}$ (LSMO) and the layered compound $\mathrm{La}_{2-2 x} \mathrm{Sr}_{1+2 x} \mathrm{Mn}_{2} \mathrm{O}_{7}$. Segregation effects, which are well-known in manganite thin films [183, 213], lower the effective doping at the surface and can result in the reconstruction of the surface layer, forming a Ruddleson-Popper-like phase [37] or in a change of the manganese valence, as shown in $\mathrm{La}_{1-x} \mathrm{~Pb}_{x} \mathrm{MnO}_{3}$ [38]. In the latter case, the valence change was attributed to the possible higher oxidation states of $\mathrm{Pb}$, compared to $\mathrm{Sr}$ or $\mathrm{Ca}$. These extrinsic segregation effects are related to oxygen deficiencies and, thus, depend on the growth conditions, i.e. temperature and oxygen partial pressure [87, 215]. They provide a serious obstacle for the study of manganite surfaces, since they alter the surface chemistry significantly and, sometimes, in an irreversible way. As a result, despite an enormous experimental effort, the theoretical predictions have not been proven and no mature picture of the surface nature has been created up to now.

Over the last twenty years, Raman spectroscopy was established as a powerful tool to study correlated materials [57], including mixed-valence manganites [63, 65, 66]. Numerous Raman studies engaged in the correlation of the observed Raman modes to the presence of Jahn-Teller (JT) distortions in the manganite system [58, 59] as well as in their relation to the metal-insulator and ferromagnetic-paramagnetic phase transition [58, 66]. In the Raman spectra, these JT distortions, which are inherent for the orthorhombic (Pnma) structure of the lightly-doped manganites $(\mathrm{x}<0.5)$, are represented by two high-frequency stretching modes at $490 \mathrm{~cm}^{-1}$ and $611 \mathrm{~cm}^{-1}$ [58, 58, 66]. In rhombohedral $\mathrm{La}_{0.7} \mathrm{Sr}_{0.3} \mathrm{MnO}_{3}$, in turn, these modes are forbidden [63, 350, 385] and instead, the Raman spectra are dominated by a sharp bending mode at $442 \mathrm{~cm}^{-1}$. Therefore, the appearance of the JT modes can be used as a finger print of the lattice structure of the manganite film. Unique Raman techniques to probe the surface structure of manganites or other correlated oxides could be Tip-Enhanced- (TERS) and/or Surface-Enhanced Raman Spectroscopy (SERS), which both exploit the excitation of surface plasmons in metallic nanostructures 
to enhance the Raman signal from nearby adsorbates [287]. The strongly localized character of the surface plasmon, i.e. its electric field $E(r)_{S P}$ scales with $1 / r^{3}$, limits the probing area down to a few nanometers $\left(d_{\mathrm{SP}} \approx 4-5 \mathrm{~nm}\right)$ [73, 74], making the study of the manganite surface by means of Raman spectroscopy in principle possible. However, up to now only few studies were performed on oxides, mostly focused on nanoparticles rather than thin films [83, 386, 387]. Moreover, to the best of our knowledge, only one TERS study on a strongly correlated material, the double perovskite $\mathrm{La}_{2} \mathrm{CoMnO}_{6}$ [84], was published, but no surface-oriented studies of the mixed-valence manganites, $\mathrm{La}_{1-x} \mathrm{~A}_{x} \mathrm{MnO}_{3}$, were reported up to now.

In this letter, we present a SERS study on thin $\mathrm{La}_{0.7} \mathrm{Ca}_{0.3} \mathrm{MnO}_{3}$ and $\mathrm{La}_{0.7} \mathrm{Sr}_{0.3} \mathrm{MnO}_{3}$ films. While the surface Raman spectra of LCMO are enhanced but congruent, the surface of LSMO reveals the dominance of two Jahn-Teller-like Raman modes. This gives evidence for the presence of the cooperative JT effect due to the symmetrybreaking-induced electron enrichment at the LSMO surface.

\subsection{Experimental Techniques}

LSMO and LCMO films have been grown by a metalorganic aerosol deposition (MAD) technique [98] on $\mathrm{MgO}(100)$ substrates (Crystal GmbH). Acetylacetonates of $\mathrm{La}, \mathrm{Sr}, \mathrm{Ca}$ and Mn were used as precursors. The films were grown at a substrate temperature $T_{\mathrm{sub}}=950^{\circ} \mathrm{C}$ with a growth rate $v=10 \mathrm{~nm} / \mathrm{min}$ and cooled down to room temperature in $15 \mathrm{~min}$. The gold nanoparticles (Au-NPs) were subsequently deposited by MAD at $T_{\text {sub }}=550-570^{\circ} \mathrm{C}$, using gold(III)acetate precursor (Alfa Aesar, $99.9 \%$ ) dissolved in dimethylformamide to a concentration $c=0.02 \mathrm{M}$. The solution with a volume, $V=1 \mathrm{ml}$, was sprayed onto the manganite film with a deposition rate $v=0.011 \mathrm{ml} / \mathrm{s}$. Finally, the prepared Au-NP/LS(C)MO $/ \mathrm{MgO}(100)$ samples were cooled down to room temperature in 10 min. X-ray diffraction in $\Theta-2 \Theta$ BraggBrentano geometry with $\mathrm{Cu}_{k \alpha}$ radiation and small-angle X-ray reflectivity measurements were performed to characterize the structure and thickness of the films. Magnetic (SQUID MPMS, Quantum design) and electrical four-probe characterizations (PPMS, Quantum design) were carried out for temperatures $T=5-400 \mathrm{~K}$. The morphology and size of the Au-NPs were characterized via scanning electron microscopy (SEM, Leo Supra 35). A four-lens optical reflection setup under normal incidence, combined with a UV-VIS spectrometer (Maya 2000Pro USB, Ocean Optics), was used to determine the wavelength of the surface plasmon resonance $\lambda_{\mathrm{SPR}}$. The Raman spectra were acquired in a back-scattering geometry with a confocal Raman microscope (LabRAM HR Evolution, Horiba Jobin Yvon) equipped with a thermoelectrically cooled charge-coupled device of 1024x256 pixels. A continuouswave HeNe-laser, $\lambda=632.8 \mathrm{~nm}$, with a spot size of $d<1 \mu \mathrm{m}$ was used and the laser power at the surface was kept at $P_{0}=0.65 \mathrm{~mW}$ during the measurement. 


\subsection{Results \& Discussion}

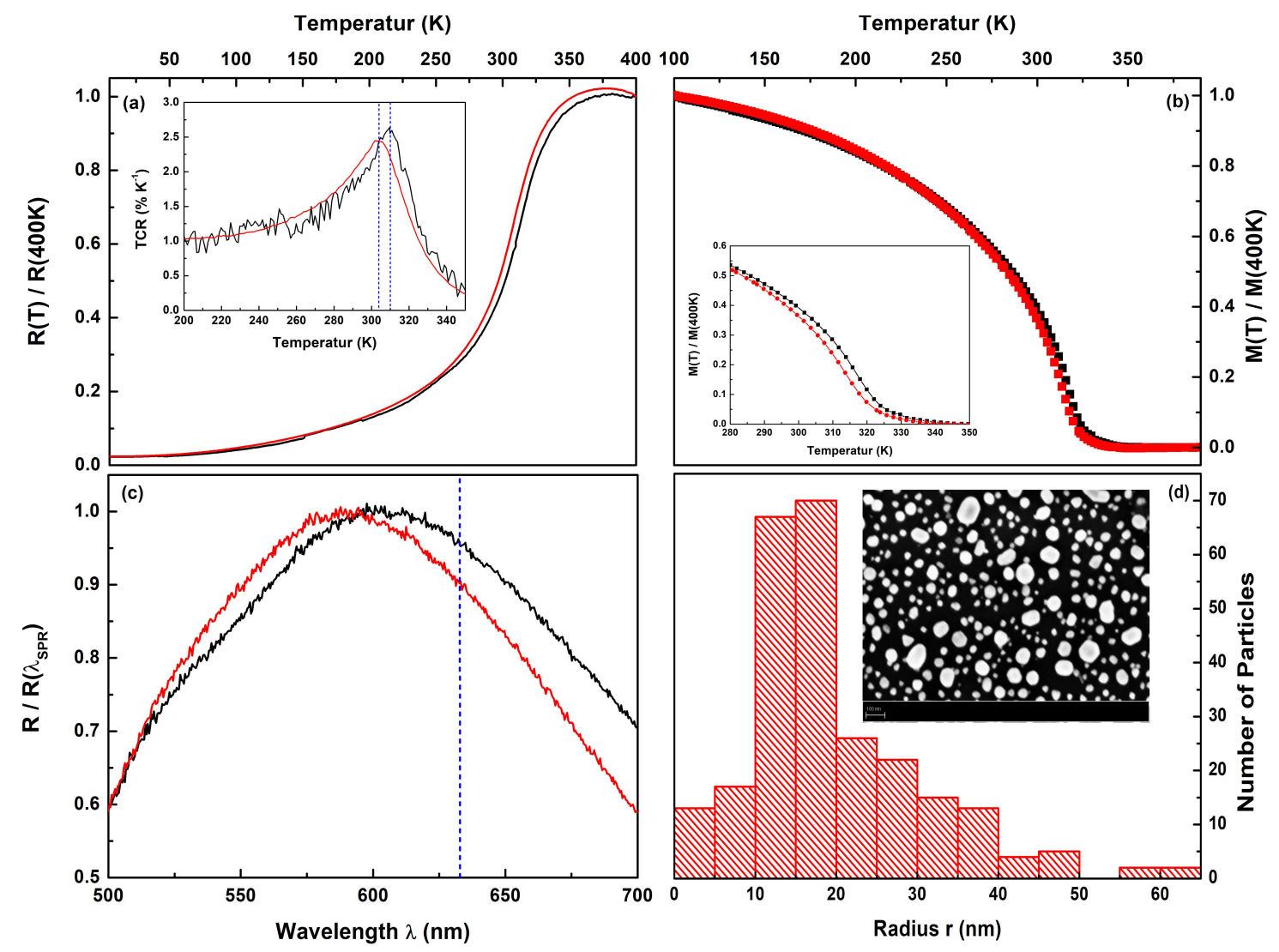

Figure 6.1.: Electrical (a) and magnetic (b) characterization of the ultrathin LSMO film $(d=12$ u.c.). Slight changes of the magnetic and electric transition temperatures infer negligible deoxygenation effects (black: LSMO; red: $\mathrm{Au} / \mathrm{LSMO}$ ). Reflectance measurements (c) provide a SPR wavelength $\lambda_{S P R}=606 \mathrm{~nm}$ for LCMO (black) and $\lambda_{S P R}=592 \mathrm{~nm}$ for LSMO (red), respectively. SEM pictures of an exemplary $\mathrm{Au} /$ manganite structure show the formation of Au-NPs with a mean particle size, $d_{\mathrm{NP}} \approx 15-$ $16 \mathrm{~nm}$, obtained from the particle size distribution (d).

Structural, electrical and magnetic characterization of thin LCMO and LSMO films are shown in Fig.S1.1-S1.4 in the Supplementary Information (SI) SI.I (see appendix $\mathrm{B}$ of this thesis) demonstrating a high quality of our thin manganite films. The estimated pseudo-cubic lattice constants, $a_{\mathrm{LCMO}}=3.867 \AA$ and $a_{\mathrm{LSMO}}=3.882 \AA$, are very close to the corresponding bulk values [99, 222] indicating a strain-free state of the manganite films on $\mathrm{MgO}(100)$. Electrical and magnetic measurements reveal a coupled ferromagnetic-paramagnetic and metal-insulator transition, $T_{\mathrm{MI}, \mathrm{C}}=267 \mathrm{~K}$, for LCMO and a ferromagnetic-paramagnetic transition, $T_{\mathrm{C}}=364 \mathrm{~K}$, and metalmetal transition, $T_{\mathrm{MM}}=343 \mathrm{~K}$, for LSMO. After the deposition of the Au-layer, the structural, electrical and magnetic properties of our manganite films did not change. 
This is in line with DFT calculations [388] and XPS measurements [389] showing no chemical interaction between an Au-layer and the manganite surface. However, Brivio et al. observed a drastic decrease of the Curie temperature for ultrathin films with a thickness less than $8 \mathrm{~nm}\left[96\right.$. They attributed the observed decrease of $T_{C}$ by $\approx 150 \mathrm{~K}$ for a $4 \mathrm{~nm}$ thick LSMO film to deoxygenation effects during the deposition of the Au-layer. Oxygen vacancies, strongly affecting the properties of the manganites [86, are a well-known problem in common thin film deposition techniques using ultra-high vacuum, e.g. pulsed laser deposition. However, the high partial oxygen pressure, $p\left(\mathrm{O}_{2}\right) \approx 0.2$ bar, within the MAD prevents or, at least, minimizes the formation of oxygen vacancies during the deposition. This is supported by annealing experiments done on MAD-grown Ruddleson-Popper $\mathrm{SrO}\left(\mathrm{SrTiO}_{3}\right)_{n}$ heterostructures [100]. To verify the stability of our films with respect to deoxygenation effects caused by the Au deposition, we prepared an ultrathin LSMO film, $d=12$ u.c. $(\approx 4.6 \mathrm{~nm})$, on a $\mathrm{TiO}_{2}$-terminated $\mathrm{STO}(100)$ substrate. Since the thickness is much smaller than $8 \mathrm{~nm}$, one could expect a strong reduction of the $T_{C}$ and $T_{M M}$, because of deoxygenation. As one can see in Fig,6.1, only minimal changes of the transition temperatures, $\Delta T_{C}=3 \mathrm{~K}$ and $\Delta T_{M M}=7 \mathrm{~K}$, occur, compared to the huge decrease in previous works [95, 96]. Therefore, deoxygenation effects are negligible in our films. SEM images of the manganite surface show a homogeneous formation of Au-NPs without any indication of clustering. Nevertheless, the particle size is distributed over a rather broad range with a mean particle size, $d_{\mathrm{NP}}=15-16 \mathrm{~nm}$ (see Fig.6.1d), as it is common for a self-aggregated growth of nanoparticles [370]. The reflectance of the studied $\mathrm{Au}-\mathrm{NP} /$ manganite films shows a surface plasmon resonance (SPR) around $\lambda_{\mathrm{SPR}}=592-606 \mathrm{~nm}$, indicated by the broad reflectance peak [390] seen in Fig.6.1. (see appendix B of this thesis).

In Fig. 6.2 , the Raman spectra of thin LCMO films at room temperature are shown. A detailed explanation of the processing of the raw Raman spectra is given in SI.II (see appendix B). The spectra are dominated by a rotational mode at $228 \mathrm{~cm}^{-1}\left(A_{1 \mathrm{~g}}\right)$ and by the two broad JT stretching modes: anti-stretching at $499 \mathrm{~cm}^{-1}$ (as-JT, $A_{1 \mathrm{~g}}$ ) and stretching at $609 \mathrm{~cm}^{-1}\left(\mathrm{~s}-\mathrm{JT}, B_{2 \mathrm{~g}}\right)$, respectively [58, 559, 65]. One can also see a small contribution of the bending mode at $438 \mathrm{~cm}^{-1}\left(B_{2 \mathrm{~g}}\right)$. The remaining modes at $343 \mathrm{~cm}^{-1}\left(B_{2 \mathrm{~g}}\right)$ and $438 \mathrm{~cm}^{-1}\left(A_{1 \mathrm{~g}}\right)$ can be also assigned to the orthorhombic structure [58]. However, since the bending mode and the JT modes play an important role in the metal-insulator transition [65, 258] and the rotational mode is sensitive to structural changes, e.g. octahedral tilting due to doping [259], the focus in the following discussion will be on these four modes. A direct comparison of the "bulk" Raman spectra and the Surface-Enhanced Raman (SER) spectra of LCMO reveals only slight differences, i.e. an enhancement of the spectra and a peak shift of the rotational mode as well as of the JT stretching modes. Furthermore, an additional mode around $568 \mathrm{~cm}^{-1}$ is observable (see Fig.6.2.). A simple approach to quantify the enhancement is to calculate the intensity contrast, $C=I_{\mathrm{SERS}} / I_{\mathrm{RS}}$, with $I_{\mathrm{SERS}}$ as SER and $I_{\mathrm{RS}}$ as normal Raman intensity. The obtained intensity contrasts are summarized in Tab6.1. Remarkably, both stretching 
modes have a different sign of peak shift $\Delta \omega=\omega_{\text {SERS }}-\omega_{R S}$ : a blue shift for the as-JT mode and a red shift for the s-JT mode.

In contrast to LCMO, the most prominent features in the "bulk" Raman spectra of LSMO (Fig. $6.2 \mathrm{~b}$ ) are the rotational mode at $178 \mathrm{~cm}^{-1}\left(A_{1 \mathrm{~g}}\right)$ and the bending mode at $422 \mathrm{~cm}^{-1}\left(E_{\mathrm{g}}\right)$, whose positions are consistent with previous observations 63, 262 and lattice dynamical calculations [385. Additionally, weak contributions of the "orthorhombic" modes at $331 \mathrm{~cm}^{-1}$ and $396 \mathrm{~cm}^{-1}$ and the JT modes at $470 \mathrm{~cm}^{-1}$ and $630 \mathrm{~cm}^{-1}$ can be seen. Since these modes are forbidden in the rhombohedral structure of LSMO [350, 385], we assign their appearance in the "bulk" spectra to a small orthorhombic distortion at the film/substrate interface, which is in agreement with previous results [391, 392] (see also appendix B of this thesis). The huge strain induced by the large mismatch between the $\mathrm{MgO}(100)$ substrate $\left(a_{\mathrm{MgO}}=4.209 \AA[99]\right)$ and LSMO $\left(a_{\mathrm{bulk}}=3.889 \AA[222]\right)$ is released by the formation of misfit dislocations, located within the first $\approx 2 \mathrm{~nm}$ from the interface [99, 334]. After this defect- and strain-rich region, the film grows unstrained and almost defect free 334 with a pseudo-cubic lattice-constant, $a_{\text {film }}=3.882 \AA$, which is almost identical to the bulk-value. These misfit dislocations are responsible for the observed orthorhombic distortions. Additionally, a broad hump centered around $515 \mathrm{~cm}^{-1}$ is clearly seen in the "bulk" spectra of LSMO and corresponds to an collective plasmalike excitation [393]. The SER spectra of LSMO, shown in Fig.6.2d, reveal an additional mode at $551 \mathrm{~cm}^{-1}$ and a strong enhancement of the JT modes at $477 \mathrm{~cm}^{-1}$

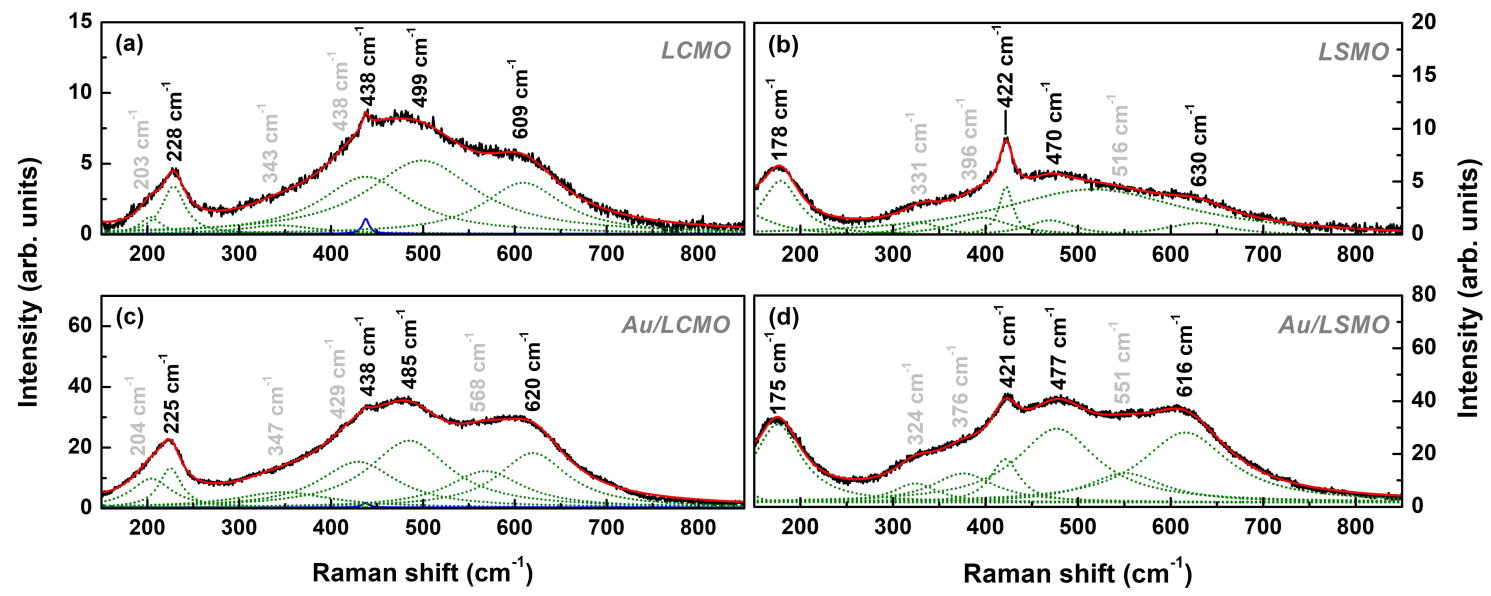

Figure 6.2.: Comparison between the "bulk" Raman spectra of LCMO (a) and LSMO (b) and their corresponding SER spectra (c, d). For LCMO (c), the Au-NP deposition results in an enhancement of the Raman spectra and an additional mode around $568 \mathrm{~cm}^{-1}$. The SER spectra of LSMO (d) show an moderate enhancement of the rotational and bending mode as well as the strong enhancement of the JT modes at $477 \mathrm{~cm}^{-1}$ and $616 \mathrm{~cm}^{-1}$, indicating a Jahn-Teller distorted surface structure. 
Table 6.1.: Intensity contrasts obtained for the different modes of LCMO and LSMO.

\begin{tabular}{c|c|c|c|c} 
& Rotational & Bending & as-JT & s-JT \\
\hline LCMO & 3.75 & 1.5 & 3.15 & 4.34 \\
LSMO & 7.35 & 7 & 34.64 & 30.52
\end{tabular}

and $616 \mathrm{~cm}^{-1}$, compared to the relatively weak enhancement of the rotational and bending mode (see Tab.6.1). However, this strong intensity enhancement cannot be attributed to an enhancement of the film/substrate interface, since the electric field of the surface plasmon decays strongly within the film. Considering the thickness of the film, $d_{\text {film }}=89 \mathrm{~nm}$, and the distance dependence of the plasmonic enhancement [73], $I \propto 1 / r^{-10}$, the intensity enhancement at the interface would be of the order of $10^{-8}$ compared to that of the surface. Hence, the observed enhancement of the JT modes in the SER spectra of LSMO is certainly a surface phenomenon. This is further supported by the observed peak shift of the JT modes. The absence of bonding partners at the surface causes a deviation of the Mn-O bond length $d_{\mathrm{Mn}-\mathrm{O}}$ [206], which will be reflected in the vibrational frequency. However, the red shift of the s-JT mode in LCMO indicates a smaller $d_{\mathrm{Mn}-\mathrm{O}}$, but the blue shift of the as-JT mode a larger $d_{\mathrm{Mn}-\mathrm{O}}$ [255]. Similar considerations apply to LSMO, but here, the JT modes are related to the film/substrate interface and not the "bulk" structure. The different sign of the peak shift suggests an asymmetric change of $d_{\mathrm{Mn}-\mathrm{O}}$ favouring the asymmetric stretching of the in-plane oxygen in LCMO, but the symmetric stretching in LSMO.

The appearance of the JT-like modes at the LSMO surface could be caused by different processes: A charge transfer from the bulk to the surface [39] would lead to the formation of an insulating $\mathrm{Mn}^{3+}$-rich layer at the surface in doped manganites with a hole concentration $x=0.3$. As a consequence, the crystal structure changes from the rhombohedral $(\mathrm{R} \overline{3} \mathrm{c}$ ) in the bulk to the orthorhombic (Pnma) structure at the surface [206]. Since the JT modes are allowed in this structure, the enrichment of the JT-active $\mathrm{Mn}^{3+}$ ion and the lowering of the symmetry should lead to the appearance of the JT modes within the insulating surface layer. Such a symmetrylowering cannot occur in an orthorhombic system, since it already resides in the lowsymmetric stable structure, predicted by the tolerance factor for perovskite oxides [394]. This is supported by theoretical calculations on orthorhombic $\mathrm{LaMnO}_{3}$ [201] and $\mathrm{CaMnO}_{3}$ [203, 205] and in good agreement with the observed enhancement in our SER spectra of LCMO. Chemical effects like segregation of the dopant [37, 183, 213, 215] are well-known for doped manganites. However, since segregation is favoured by a low oxygen partial pressure [215], these effects should be suppressed due to the high $p\left(\mathrm{O}_{2}\right)$ provided by the MAD. An enrichment of $\mathrm{Sr} / \mathrm{Ca}$ at the surface would further result in a higher effective doping at the surface, leading, thus, to a reduction of the JT modes [267] and not an enhancement. Another possible 
explanation for the appearance of the JT-like modes at the LSMO surface is the $\mathrm{Au}-\mathrm{NP}$ deposition itself. X-ray absorption spectroscopy on LSMO $(d=40 \mathrm{~nm})$ with an Au-capping layer $(d=2 \mathrm{~nm})$ revealed the presence of $\mathrm{Mn}^{2+}$ at the interface between film and capping layer [395]. Additionally, the deposition of Au-NPs onto the surface creates an interface between the gold and the manganite surface, which could cause the observed symmetry-breaking. However, the formation of $\mathrm{Mn}^{2+}$ as well as the artificially created symmetry-breaking would impact the $\mathrm{Mn}^{3+} / \mathrm{Mn}^{4+}$ ratio and thus, result in a decrease of the transition temperature. As mentioned before, even an ultrathin film does not show any significant changes of its properties after the Au-NP deposition. Therefore, the formation of $\mathrm{Mn}^{2+}$ or an artificially induced symmetry-breaking caused by the Au-NP deposition is unlikely. Hence, the JT-like modes have to be an intrinsic electronic surface phenomenon or they are related to the $\mathrm{Au}-\mathrm{NPs}$ themselves.

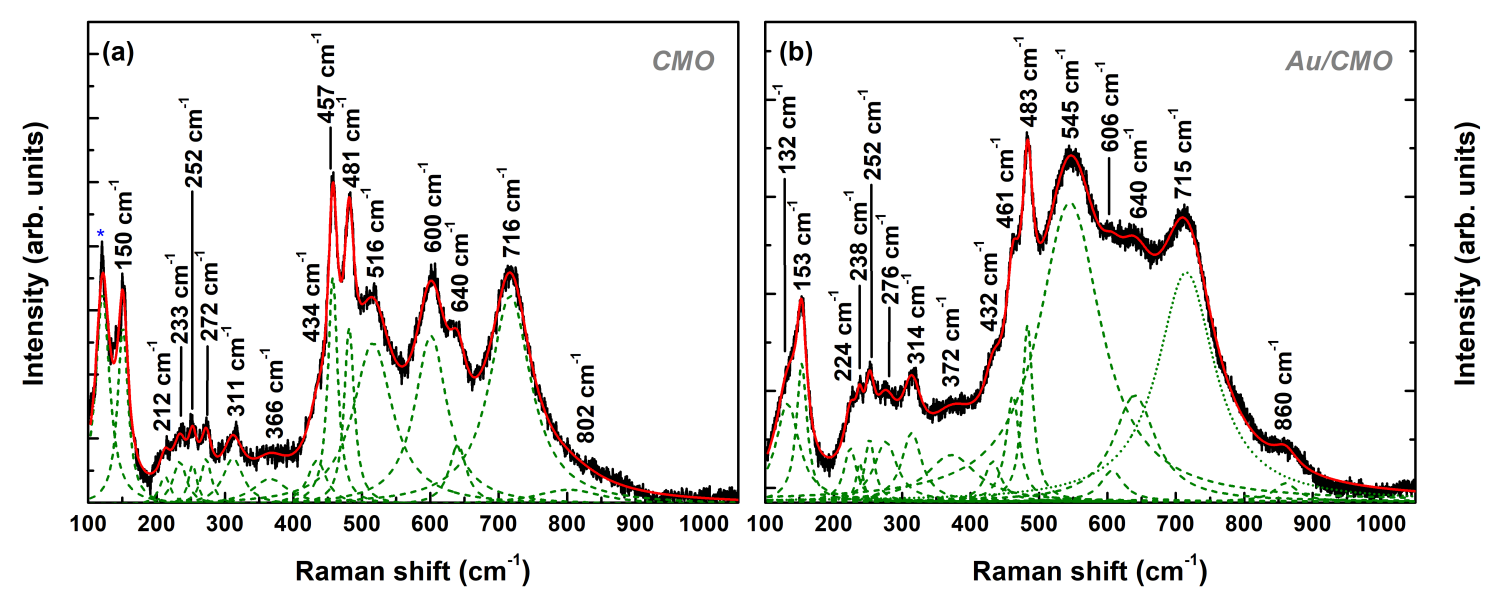

Figure 6.3.: The bulk (a) and SER spectra (b) of $\mathrm{CaMnO}_{3}$ reveal no Jahn-Tellerrelated Raman modes, supporting the authenticity of our LSMO spectra and the appearance of the Jahn-Teller stretching mode.

To verify the authenticity of the appearing JT-like modes at the LSMO surface and to exclude effects caused by the Au-NPs themselves, we have grown orthorhombic $\mathrm{CaMnO}_{3}$ thin films on the lattice-matched $\mathrm{LaAlO}_{3}$ (100) substrates (see Fig.1.5 in SI.I (see appendix B for plasmonic characterization). $\mathrm{CaMnO}_{3}$ shows a 60 times smaller JT distortion than the orthorhombic $\mathrm{LaMnO}_{3}$ and does not feature any Jahn-Teller related Raman modes (Fig.6.3 a) [396. Slab calculations of the $\mathrm{CaMnO}_{3}$ surface predict only a possible change of the magnetic order [203, 205], but no electronic or structural reconstruction, which could cause a stronger JT distortion. As one can see in Fig 6.3 b, there are no additional JT-like modes present in the SER spectra of $\mathrm{CaMnO}_{3}$. This verifies the authenticity of the observed JT-like modes in the SER spectra of LSMO and excludes their appearance caused by the Au-NPs 
themselves 11. However, as in LCMO and LSMO, an additional mode at $545 \mathrm{~cm}^{-1}$ is clearly visible in the SER spectra of $\mathrm{CaMnO}_{3}$ and, likely, will be of similar origin as the additional surface mode in LCMO and LSMO.

Hence, we can conclude that the strong enhancement of the JT-like modes has to be an intrinsic surface phenomenon of the LSMO surface and that, they can, indeed, be assigned to the JT stretching vibrations, which are inherent to the orthorhombic (Pnma) structure of the lightly-doped manganites. Note that, we assume a $\mathrm{MnO}_{2}{ }^{-}$ terminated surface of our manganite films as observed in previous works [209] [208]. But, since the surface termination of the studied films is unknown, one has to keep in mind, that a different termination of the manganite surface could lead to an alternative surface reconstruction and thus, to the suppression of the JT modes. Nonetheless, we can experimentally confirm herewith the theoretically predicted symmetry lowering at the manganite surface and the formation of an electron-rich JT distorted surface layer in the ferromagnetic metallic LSMO as well as the electron enrichment at the surface of the orthorhombic LCMO.

To model the surface structure of LSMO and LCMO, we compared the intensity contrast of the different modes (see Tab 6.1). By keeping in mind the strong distance dependence of the plasmonic enhancement [73], the significantly higher contrast of the JT modes in comparison to the bending mode can be directly linked to a stronger surface contribution of them. Hence, the bending mode can be considered as a "bulk" mode and can be taken to estimate the thickness of the Jahn-Teller distorted surface layer. The treatment as a "bulk" mode is further supported by its negligible peak shift, which would be expected from an undisturbed structure. Using the simplified sphere model and the $|E|^{4}$ approximation, the SER intensity scales with [73]

$$
I_{S E R S} \sim[(1+r / a)]^{-10}
$$

with $I_{S E R S}$ the SER intensity of the Raman mode, $a$ the radius of curvature of the field enhancing feature of the metallic surface and $r$ the distance from the metallic surface to the adsorbate. Assuming the radius of curvature is given by the mean particle size, $d_{\mathrm{NP}} \approx 16 \mathrm{~nm}$, Eq.1 yields the distance dependence shown in Fig.6.4. Since we consider the bending mode as a bulk mode, the ratio $\eta=C_{\text {bend }} / C_{\mathrm{JT}}$ represents the weakening of the plasmonic enhancement within the whole distorted surface layer. To estimate the thickness of the surface layer, one compares the determined ratio with the distance dependence in Fig.6.4. For LSMO, the determined ratio, $\eta \approx 7 / 31$, yields a thickness of the surface layer, $d_{\mathrm{JT}} \approx 7$ u.c. $\approx 2.8 \mathrm{~nm}$. Similar considerations for the modes of LCMO yield a significantly smaller thickness $d_{\mathrm{JT}} \approx 4-5$ u.c. $\approx 1.7-2.0 \mathrm{~nm}$. Both values correspond well to the thicknesses of the

1 One can observe a change in the intensity ratios of the modes, which can be discussed in a picture of a distorted surface structure. Every mode is caused by a specific rotation of the $\mathrm{MnO}_{6}$ octahedron, so that a different intensity ratio gives information about the preferred octahedral tilting at the surface. However, this discussion would be beyond the scope of this paper and will be discussed elsewhere. 
insulating antiferromagnetic surface layer obtained from XMCD [93] and XRMS [103. The insert in Fig.6.4 summarizes the proposed model of the manganite surface. Note that the intensity contrast of the rotational mode in LSMO is only slightly higher than the contrast of the bending mode, indicating a rather bulk-related than surface-related nature. In LCMO, however, the intensity contrast is similar to those of the JT modes and thus, will be associated to the surface structure. As mentioned before, this mode is sensitive to the tilting of the $\mathrm{MnO}_{6}$ octahedron, so that its blue shift suggests a change of the octahedral tilting near the surface. However, both manganite systems show a comparable peak shift and therefore, a similar octahedral tilting. Considering that a structural transition occurs at the LSMO surface, this is quite remarkable and, in combination with the slightly higher contrast of the rotational mode, hints the presence of an intermediate layer, which accommodates the structural transition at the surface.

Finally, we want to discuss the assignment of the additional mode around 550-
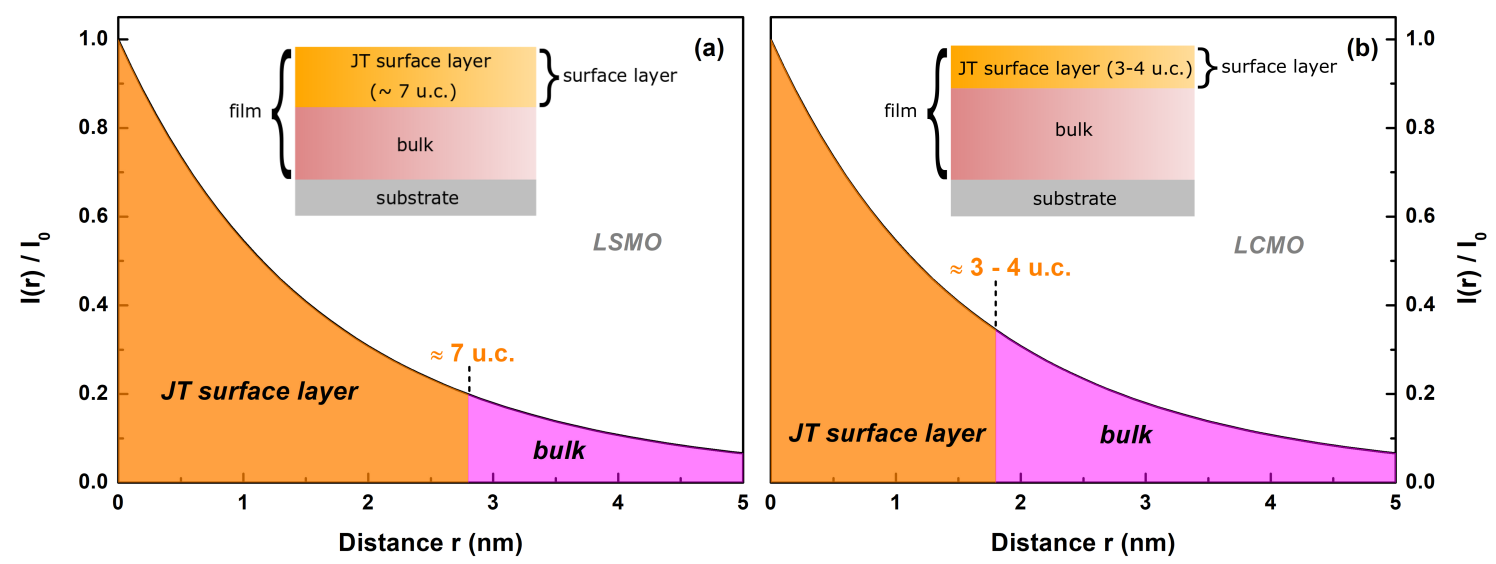

Figure 6.4.: The modelled (a) LSMO and (b) LCMO surface structure, determined from the distance dependence of the electromagnetic enhancement due to the plasmonic excitation in the Au-NPs.

$570 \mathrm{~cm}^{-1}$. Compared to theoretical calculations [58, 396, the closest mode would be the out-of-phase stretching mode at $536 \mathrm{~cm}^{-1}$ in $\mathrm{CaMnO}_{3}$ and $575 \mathrm{~cm}^{-1}$ in $\mathrm{LaMnO}_{3}$ of $B_{1 \mathrm{~g}}$ symmetry, respectively. Although, our back-scattering geometry only allows the detection of the $A_{1 \mathrm{~g}}$ and $B_{2 \mathrm{~g}}$ modes, one can imagine that the symmetry-breaking at the surface and the rearrangement of the $\mathrm{MnO}_{6}$ octahedron could result in a structural distortion at the surface allowing the observation of $B_{1 \mathrm{~g}}$ modes. Further studies, especially from the theoretical point of view, could give insight into the origin of the additional mode and our proposed assignment.

\subsection{Conclusion}

We have studied the surface structure of the mixed-valence perovskite manganites by means of SERS. The SER spectra reveal an electron-rich surface structure, which 
is manifested by the dominance of the JT stretching modes. The strong enhancement of the JT modes in LSMO was attributed to a structural transition to the orthorhombic (Pnma) structure at the surface, which results in a unique surface structure. This is the direct experimental confirmation of the theoretically predicted surface of the doped manganite with $x=0.3$. The authenticity of our SERS results obtained for the rhombohedral LSMO is supported by the SER spectra of orthorhombic LCMO and CMO.

\subsection{Acknowledgment}

The authors acknowledge financial support from the Deutsche Forschungsgemeinschaft (DFG) via SFB 1073 (TP B04, B01 and A02). 


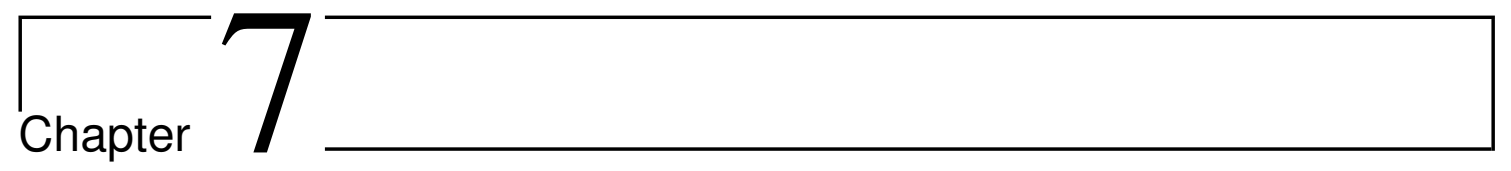

\section{Conclusion}

\subsection{General Discussion}

In the following chapter, the results presented previously shall be generally discussed and embedded into the current state of manganite physics. Its emphasis will lie in the polaronic contributions to the different phenomena occurring in mixed-valence manganites. The general contribution of polarons to the emerging symmetry-breaking at interfaces will be pointed out, whether it is of structural nature, such as at macroscopic interfaces, or of electronic nature, such as at the microscopic interface between the electronically separated phases. Chapter 5 describes methodical advances needed for the facilitation of SERS on correlated oxides and, therefore, takes on a special role within this these. For this reason, the results presented in that chapter will be left out of the following discussion.

As a starting point, the nature of the MI transition and CMR will be further analyzed. Chapter 3 and 4 engage in the temperature- and magnetic-field-dependent behavior of the Jahn-Teller polarons and their correlations to the MI transition and CMR. As mentioned in Ch.2.4, ILIEv et al. pointed out [65] that, the doping of manganites with alkaline earth metals induces a mixed-valence state, disturbing the cooperative JT effect. The $\mathrm{Mn}^{4+}$ ions induced to the manganite system can be therefore considered as a lattice defect, which causes a broadening of the Jahn-Teller modes. In this case, the JT stretching modes do not represent $\Gamma$-point phonons anymore, but rather have their origin in the phonon density of states of the oxygen ions and express the degree of disorder in the oxygen sublattice. Hence, any changes in the intensity of the JT modes constitute a change in the oxygen disorder and, consequently, in the octahedral $\mathrm{MnO}_{6}$ network. Considering the strong electronphonon coupling in the LPCMO system and the resulting substantial disorder (see Ch.2.1.2), the MI transition as well as the CMR may be described as disorder-order transitions of the octahedral network. Therefore, the Raman intensity behavior presented in Ch.3 and 4 [66, 268] can be interpreted in the following way: first of all, the strong Raman intensity increase of the JT modes in the vicinity of $T_{\mathrm{C}}$ indicates 
a rapid increase of the $\mathrm{JT}$ distortion in the manganite system. This is supported by the enhanced signal intensity in the $3 \omega$ measurements. The nonlinear $3 \omega$ signal is a general measure for the amount of correlated polarons [149], thus, the increasing signal indicates a raised amount of correlated polarons. However, the hole doping remains formally the same (i.e. for $\left(\mathrm{La}_{0.65} \mathrm{Pr}_{0.45}\right)_{0.7} \mathrm{Ca}_{0.3} \mathrm{MnO}_{3}$ this would be $y=0.45$ and $x=0.3$, in $\mathrm{La}_{0.7} \mathrm{Ca}_{0.3} \mathrm{MnO}_{3}$ it would be $x=0.3$ and so on). This is why, the higher amount of polarons cannot be explained by a higher portion of Jahn-Teller active $\mathrm{Mn}^{3+}$ ions. Such an intensity increase can rather be explained by an elevated disorder in the system, which is mediated by an increased amount of localized charge carriers due to the formation of correlated polarons. At higher temperatures, small polarons move freely through the lattice by thermally-activated hopping [131, 136141]. When approaching the MI transition, more and more of the small polarons are bound into pairs by the short-ranged correlations, forming strongly localized bipolarons [146]. The pairs of small polarons are now bound to a specific lattice site, and may not move freely through the lattice anymore. Therefore, they can be considered as additional lattice defects, increasing the disorder in the system. Since the polarons are related to the $\mathrm{MnO}_{6}$ octahedral network, the increasing amount of correlated polarons can be viewed as a disturbance of the octahedral network and, hence, of the oxygen sublattice in the perovskite unit cell. This is ultimately reflected in the increased Raman intensity of the JT modes.

The strong localization of charge carriers raises the electrical resistivity resulting in a highly insulating state at $T_{\mathrm{C}}\left(\rho>10^{-1} \Omega \mathrm{cm}\right)$, which represents a highly disordered state. Consequently, by suppressing the polaronic phase via an applied magnetic field [268] or lower temperatures [65, 66], the amount of correlated polarons is decreased and the bipolaronic structure falls apart. This results in a lower oxygen disorder, which is directly reflected in a lower Raman intensity of the JT modes [66, 268] and a smaller $3 \omega$ signal [66]. Since the short-range polaronic correlations within the manganite system decline, the charge carriers are less strongly bound, leading to their delocalization and the enhanced conductivity [66, 268]. The observed collapse of correlated polarons and the resulting enhancement of the electrical resistivity can be attributed to a higher energy gain caused by the onset of ferromagnetic order, which overcomes the energy gained by forming bipolarons due to the strong electron-phonon coupling. In this context, however, it should be kept in mind that the charge carriers do not lose their polaronic character [133-135] and the transport may be carried out by large polarons. In a simple picture, large polarons can be considered as delocalized small polarons [132, 134, 135], thus, forming a polaronic metal.

The elevated structural order is further demonstrated by the rising intensity of the sharp $\Gamma$-point phonon around $440 \mathrm{~cm}^{-1}$ [65], representing a bending mode of the oxygen octahedron (see Ch 2.2 . . While its intensity is nearly constant for temperatures of $T>T_{\mathrm{C}}$, a monotonic intensity increase can be observed below $T_{\mathrm{C}}$, which is in line with previous Raman studies of mixed-valence manganites [65]. Because 
of the dominance of the bending mode within the ferromagnetic metallic regime, it can be viewed as a fingerprint of the same. As seen in Fig. 3.7, the intensity behavior of the bending mode and the JT modes can be summarized in a $I_{620} / I_{440}$ vs. $\left(T-T_{\mathrm{C}} / T_{\mathrm{C}}\right)$ plot, revealing a pronounced interplay between both modes. It further illustrates the competition between the polaronic disordered and the ordered polaron-free ferromagnetic metallic structure and its importance for the MI transition, since the JT mode is significantly enhanced at $T_{\mathrm{C}}$. In general, such an interplay of two Raman-active vibrational modes can indicate a structural phase transition in terms of a change of the lattice symmetry. This conjecture is supported by temperature-dependent Raman spectra of rhombohedral $\mathrm{La}_{0.7} \mathrm{Sr}_{0.3} \mathrm{MnO}_{3}$ (LSMO) 393. Generally, LSMO can be considered as a well-ordered manganite structure due to the missing JT distortions [180]. Its Raman spectra are dominated by the rotational and the bending mode (see Ch.2.2.2) describing similar vibrations to the ones observed in orthorhombic manganites [58, 59, 350]. Decreasing the temperature results in a monotonic growth of the intensity of these modes [393], which is similar to the observed behavior in $\mathrm{La}_{0.7} \mathrm{Ca}_{0.3} \mathrm{MnO}_{3}$ (LCMO) or LPCMO below $T_{\mathrm{C}}$. Hence, it was assumed that the ordered low-temperature phase is represented by the rhombohedral $(\mathrm{R} \overline{3} \mathrm{c})$ structure. However, such a structural phase transition in LPCMO would be reflected in a symmetry change of the bending mode (from a $E_{\mathrm{g}}$ symmetry in the rhombohedral [258] to $B_{2 \mathrm{~g}}$ symmetry in the orthorhombic structure [59]). Following the selection rules given in Ch.2.2, the Raman line could not be assigned to the $E_{\mathrm{g}}$ symmetry [61] and remains of $B_{2 \mathrm{~g}}$ symmetry. This excludes a structural phase transition below $T_{\mathrm{C}}$ and, therefore, the presented results support the assumption of an order-disorder transition.

As shown in the temperature- and magnetic-field-dependent Raman measurements in Fig 3.7 in Ch.3 and Fig.4.3 in Ch.4 respectively, the strongly disordered state manifests itself only in a narrow temperature range. Additionally, the phase diagram in Fig 4.4 reveals that the CMR and MI transition develop within the same temperature region [268, indicating that they are driven by the described disorder-order transition. This disordered state is further characterized by an antiferromagnetic coupling between the ferromagnetic nano-domains [66]. Such coupling is mediated by correlated polarons and becomes apparent by the increase of the coercive field by approaching $T_{\mathrm{C}}$ (see Ch.3). Beyond this temperature regime $\left(T_{\mathrm{C}}>T>T^{*}\right)$, the amount of correlated polarons is roughly constant, which is insinuated by the constant Raman intensity of the JT modes [66, 268, the non-zero $3 \omega$ signal [66] and neutron scattering experiments [145]. Considering the substantial disorder in the CMR region, $T_{\mathrm{C}}>T>T^{*}$ [268], one can refer to the present phase as a polaronic glass [1, 144, 160]. This is supported by the nature of correlated polarons as short-ranged correlations. Conceptually, these correlations are comparable to the short-range correlations/order in an amorphous glass and, thus, the suppression of the polaronic phase is often referred to the melting of the polaronic phase [397]. A short-range order induces a broadening of the Raman modes due to the imperfect crystalline lattice. Furthermore, the first-order Raman scattering in 
amorphous materials is described in terms of the density-of-states [49]. Both are directly reflected in the measured Raman spectra of LPCMO and, therefore, give further evidence of the glassy nature of the CMR phase.

As mentioned in Ch.2.1.2, the electron-phonon coupling can be tuned by chemical doping [180] and induces the discussed disorder in the manganite system due to the binding of the electron to the lattice. Moreover, the quenched disorder is believed to cause the electronic phase separation [152, 155, 157, 159]. Since the sharp bending mode can be considered as a sign for the metallic phase in LPCMO [65, 66, 268, 393, the low intensity of the bending mode in the paramagnetic-insulating phase as well as the remnants of the JT modes in the low-temperature ferromagnetic-metal phase [65, 66, 268] support the picture of electronic phase separation in LPCMO. The substantial disorder induced by the strong electron-phonon coupling, indeed, opens a gap between the two ordered states, i.e. the ferromagnetic-metallic and the charge/orbitally ordered antiferromagnetic phase, causing the glassy phase separated intermediate phase [159], where CMR and metal-insulator transition develop [157, 159, 268. In this state, both ordered phases compete strongly with each other [159]. Such competition is believed to be fundamental for the high values of the CMR in strongly electron-phonon coupled systems [159]. The importance of the competition and, in particular, of the charge/orbitally ordered phase, i.e. the formation of correlated polarons, can be seen also by comparing the strength of the magnetoresistance in LPCMO and LCMO. The relatively high transition temperature of LCMO, $T_{\mathrm{C}, \mathrm{LCMO}}=260 \mathrm{~K}$, is well-above the typical charge-ordering temperature $T_{\mathrm{CO}}=220 \mathrm{~K}$ in highly doped $\mathrm{La}_{1-x} \mathrm{Ca}_{x} \mathrm{MnO}_{3}(x>0.5)$ [398] or $\mathrm{Pr}_{0.7} \mathrm{Ca}_{0.3} \mathrm{MnO}_{3}$ [399]. At this temperature, the formation of small CO islands starts and, therefore, the formation of correlated polarons. Although the JT distorted LCMO system exhibits a MI transition and electronic phase separation, the intermediate electron-phonon coupling is not sufficient enough to pull the transition temperature below $T_{\mathrm{CO}}$. The missing charge-ordering effects result in a low amount of correlated polarons, which is too low to mediate AFM coupling. This results in a relatively small magnetoresistance of $\mathrm{MR} \approx 100 \%$, which is even lower in the well-ordered LSMO [22, 131, 149]. The importance of charge-ordering for CMR is further demonstrated by signs of instability in the LPCMO system with respect to an applied magnetic field below $T_{\mathrm{CO}}$, causing metamagnetic transitions [66]. Below $T_{\mathrm{CO}}$, the antiferromagnetic coupling between the ferromagnetic domains, mediated by correlated polarons, can be cracked by applying a critical magnetic field of more than $H=20 \mathrm{kOe}$, which enhances the magnetization and electrical conductivity. The Raman spectra shown in Ch.3 and $\mathrm{Ch} 4$ support the assumption of arising instabilities in strongly electron-phonon coupled manganite systems, since first temperature- and magnetic-field-induced effects can be observed below $T_{\mathrm{CO}}$ (see Fig, 4.3 and Fig, 4.5). Furthermore, the competition between the charge-ordered polaronic phase (represented by the JT modes) and the ordered polaron-free phase (represented by the bending mode) is directly reflected in the enhancement of the JT mode at $T_{\mathrm{C}}$ (see Fig.3.7). Therefore, the presented results support the common believe that the glassy phase separated state 
and the competition of both of the phases cause the high CMR values in strongly electron-phonon coupled systems [180], such as LPCMO [164]. Furthermore, the quantitative resemblance of the obtained magnetic-field-induced intensity decrease of the JT modes of $\Delta I=6000 \%$ (see Ch.4 and [268]), and the obtained CMR value of $7000 \%$ in LPCMO, show the significance of correlated polarons for the CMR.

Since the discussed disorder-order transition is commonly observed in manganites [64, 331], it presumably represents a fundamental contribution to manganite physics. In this regard, an interesting question may be, where exactly the correlated polaron is located within the phase separated material. As discussed in Ch.2.1.3. theoretical calculations predict an enrichment of $\mathrm{Mn}^{3+}$ ions, i.e. a possible polaron accumulation, at the surface [39] and at interfaces [352]. In general, an electronically phase separated material, e.g. LPCMO, can be considered as a material with many internal boundaries/interfaces, which are induced by the symmetry-breaking due to the dissimilar electronic properties of the two ordered phases. Therefore, a possible location spot of correlated polarons may be the domain walls of the ferromagnetic metallic domains. Such conjecture is supported by the small charge correlation length of the antiferromagnetic CO phase of only a few unit cells, $\sqrt{5} a_{\text {per }}$ (with $a_{\text {per }}$ as the lattice constant of the perovskite structure) [159], which is nearly identical to the polaronic correlation length of $\delta \approx 1-2 \mathrm{~nm}$ from scattering experiments [144, 146, 336, 400]. Along with the estimated size of the ferromagnetic metallic domains of $6-8 \mathrm{~nm}$ and the average distance between the correlated polarons of $9 \mathrm{~nm}$ (see Ch.3 and Ref.[66]), these observations highly suggest a preferred nucleation of correlated polarons at the electronic interfaces within the manganite system, i.e. at the boundaries of the metallic domains. Indeed, a theoretical basis for this conjecture is given by BREY, who analyzed the manganite interface in terms of an electrostatic barrier caused by the abrupt change of charge carrier concentration [352. The electron depletion at the interface leads to the formation of a CE-type phase, which stabilizes within a thin interfacial layer of 3 u.c. thickness. Such an interfacial layer of CE-type would match the structure of correlated polarons and further indicates their preferred nucleation at the microscopic interface.

This preferred formation at an interface could be further verified by the SERS study presented in $\mathrm{Ch} 6$. The strong enhancement of the JT stretching vibrations compared to the bending mode indicates the formation of $\mathrm{Mn}^{3+}$ ions and, thus, electron accumulation at the LCMO and LSMO surface. Additionally, the symmetrybreaking and the resulting electron enrichment at the surface presumably leads to a symmetry-lowering from a rhombohedral to an orthorhombic structure at the LSMO surface, which can be deduced from the irreducible representation of both structures. While the rhombohedral structure only allows five Raman-active modes [258, 350], the orthorhombic one gives rise to 24 Raman-active vibrations [58, including the JT stretching vibrations. Since these modes have their origin in a present JT distortion [58, 59, 262] and, thus, should be forbidden in the LSMO structure [258, 350], their strong enhancement has to arise from a structural phase transition at the surface. 
Furthermore, the removal of the topmost oxygen ions at the surface [39, 206] induces disorder in the oxygen sublattice and the $\mathrm{MnO}_{6}$ network, respectively. The oxygen disorder is reflected in the Raman frequency shift of the JT modes [401], since the missing oxygen ions change the Mn-O-Mn bond angles as well as the Mn-O bond lengths at the surface, compared to their bulk values [206]. Therefore, these experiments indicate huge similarities between the microscopic interfaces (i.e. domain boundaries) within the phase separated manganite system and the macroscopic interfaces located at the surface and film/substrate interface. Both are characterized by a preferred polaron nucleation and a high disorder of the oxygen sublattice. However, the polaron structure at the surface will likely be different to the polaronic structure in the vicinity of $T_{\mathrm{C}}$. As aforementioned, the formation of correlated polarons starts below the typical charge-ordering temperature $T_{\mathrm{CO}}=220 \mathrm{~K}$. Since the SERS experiments are performed at ambient temperatures and pressures, the $\mathrm{CO}$ clusters have not yet been formed and the polarons may be arranged in a semicooperative structure, similar to low-doped $\mathrm{La}_{1-x}(\mathrm{Ca}, \mathrm{Sr})_{x} \mathrm{MnO}_{3}(x \leq 0.4)$ [351, 402 ] and undoped LMO [58, 59]. Furthermore, the native water layer at the surface may effect the observed JT distorted surface as well, since its influence cannot be accurately determined.

Overall, the experimental results presented in this thesis indicate a preferred nucleation of polarons at electronic and structural interfaces. This is further supported by XMCD and photoemission experiments [41-43], indicating similar electron accumulation processes at the film/substrate interface. Comparing the electronic accumulation at both macroscopic interfaces, i.e. the surface and the film/substrate interface, it becomes apparent that the thickness of the electronically distorted layers are nearly identical, $d \approx 7$ u.c. in LSMO [41, 43, 401] and $d \approx 4$ u.c. in LCMO [401, 403]. Note that, the barrier at the LSMO surface is twice as large as in LCMO, which is probably caused by an additional compensation of the occurring structural phase transition, which is negligible in the orthorhombic LCMO. Remarkably, the characteristic length scales of the distorted macroscopic interface layers, the correlation lengths of correlated polarons [144, 146, 336, 400] and the charge localization length in the antiferromagnetic insulating phase [159] are of similar magnitude and fit quite well with the theoretical predicted accumulation length of 3 u.c.. Therefore, it can be assumed that the compensation of the symmetry-break, whether it is of structural or electronic nature, comes from the nucleation of (correlated) polarons at the corresponding interface. However, this charge accumulation and charge-ordering provides an additional electrostatic barrier at the interface. While it may minimize the polar discontinuity occurring at the macroscopic interface, it gives rise for electrical and magnetic dead layers [28]. The strongly localized charge carriers suppress the double-exchange mechanism and establish an antiferromagnetic order, which results in the magnetic dead layer. Presumably, the antiferromagnetic order at the manganite surface will be similar to a C-type antiferromagnet [211, 226]. The missing oxygen ions along the [001] direction will lead to a lowering of the $3 \mathrm{z}^{2}-\mathrm{r}^{2}$ orbital due to the lower Coulomb repulsion [39]. This is similar to the case of an applied com- 
pressive strain, which causes a similar orbital polarization due to the compression of the $a b$-plane and the elongation of the $c$-axis [211, 226]. Theoretical calculations by CALDERón proposed a canted-antiferromagnetic order at the surface, which can be broken by an extremely high magnetic field, $B>60 \mathrm{~T}$ [39]. Therefore, the electrostatic barrier provided by the accumulating polarons and the resulting antiferromagnetic order at the surface may reasonable explain the vanishing low-field magnetoresistance in tunnel magnetoresistive devices at room temperature [32, 404].

Polaron formation and the corresponding disorder-order transition further support experiments performed by conductive atomic force microscopy [187, 240, 405], which induced a low resistive metallic state by applying an electric field. The metallic surface state obtained was remanent and, furthermore, a reversible switching between the low resistive metallic and high resistive insulating state was shown. While the mechanism behind such electric field induced resistive switching is often assumed to be oxygen diffusion, KRISPONEIT et al. [187] have shown that the switching likely results from a structural phase transition. This is indicated by the occurrence of reversible switching between the two resistive states at low temperatures, at which the oxygen diffusion will be too low to mediate the switching [187]. Instead, they attributed the switching process to a structural phase transition from an initial polaronic insulating CE-type phase to a polaron-free metallic phase, which corresponds to the discussed disorder-order transition observed in the temperatureand magnetic-field-dependent Raman spectra. Furthermore, the polaron accumulation at the surface demonstrated in Ch.6 supports the suggested polaron-mediated coupling of the c-AFM tip to the electric field and the proposed nature of the high resistive state [187]. Additionally, the estimated barrier height of $1.3 \mathrm{~nm}$ [187, 240] agrees well with the thicknesses of the nonmagnetic/insulating layers obtained in XMCD experiment [41, 43, 103, 395] and in the presented SERS measurements (see $\mathrm{Ch} .6)$.

Along the discussed surface/interface phenomena, the charge accumulation/charge transfer seem to be responsible for emerging interface phases in manganite superlattices. Recently, a high- $T_{\mathrm{C}}$ phase in $\mathrm{LaMnO}_{3} / \mathrm{SrMnO}_{3}$ superlattices has been discovered [101], which results from a charge transfer from the electron-rich LMO to the electron-poor $\mathrm{SrMnO}_{3}(\mathrm{SMO})$. Similar to the polaron nucleation at the surface, this interfacial charge transfer is driven by the compensation of the polar discontinuity (see Ch.2.1.3 [101]. By monitoring the superlattice growth in-situ by means of optical ellipsometry, an electron-rich region at the LMO/SMO interface with a thickness of approximately 2 u.c. was observed. The charge injection into the SMO layer presumably results in an interfacial ferromagnetic insulating phase [101]. The charge transfer length of the superlattice fits well to the previously discussed polaronic correlations lengths, thus, indicating a characteristic length, which is needed to compensate the polar catastrophe at manganite interfaces. The possible occurrence of an interfacial ferromagnetic insulating phase may provide an interesting starting point for further SERS studies on manganites. Low-doped $\mathrm{La}_{1-x} \mathrm{Sr}_{x} \mathrm{MnO}_{3}$ with 
$0.1<x<0.175$ shows a similar ground state [406 408] and corresponding Raman spectra are dominated by polaronic excitations [351. The high sensitivity of SERS may provide further insight into the nature of the interfacial layer and may verify the existence of the suggested ferromagnetic insulating phase.

\subsection{Summary and Outlook}

In summary, this thesis pointed out the significance of interfaces for the understanding of the fundamental processes in manganites, such as CMR and metalinsulator transition, as the polarons accumulate at the inner domain boundaries. Furthermore, the observed polaron accumulation at the macroscopic interface, i.e the surface, offers an additional electrostatic barrier and, therefore, contributes to the formation of dead layers.

The first two publications of this cumulative thesis engaged in the temperatureand magnetic-field-dependent Raman intensity behavior of the strongly electronphonon coupled LPCMO. An increasing amount of correlated polarons in the vicinity of $T_{\mathrm{C}}$ was proposed as the underlying mechanism behind the MI transition, which is supported by the increased intensity of JT modes at the transition temperature and the enhanced $3 \omega$ signal in nonlinear electrical resistivity experiments. Furthermore, the abnormal behavior of the coercive field suggests an AFM coupling between the nanometer-sized ferromagnetic domains $\left(R_{\mathrm{FM}} \approx 6-8 \mathrm{~nm}\right)$. The short correlation length $(\delta \approx 1-2 \mathrm{~nm})$ and the average distance between the correlated polarons $\left(A_{\mathrm{CP}}=9 \mathrm{~nm}\right)$ indicate that the antiferromagnetic coupling is mediated by correlated polarons. Similar length scales of $R_{\mathrm{FM}}$ and $A_{\mathrm{CP}}$ revealed further a preferred location at the ferromagnetic domain boundaries. Apparently, only a tiny amount of the polarons has to be correlated $\left(n_{\mathrm{CP}}=0.5 \%\right)$ to mediate the AFM coupling between the ferromagnetic domains. An increased amount of correlated polarons promotes disorder of the oxygen sublattice in the manganite system. Its suppression in the metallic regime allows for the description of CMR and MI transition within the framework of a disorder-order transition. Such a transition is reflected in a pronounced interplay of the JT modes and a sharp $\Gamma$-point phonon mode emerging at $440 \mathrm{~cm}^{-1}$ in the temperature- and magnetic-field-dependent Raman scattering experiments presented in $\mathrm{Ch} 3$ and 4 . The observed interplay underscores the relevance of the lattice for the competition of the charge-ordered polaron-rich and the ferromagnetic metallic phase. It could also be shown that the CMR and the magnetic-field-induced Raman intensity decrease develop only within a narrow temperature range of $T_{\mathrm{C}}>T>T^{*}$, emphasizing their common origin. The outer limit of this narrow range is represented by the charge-ordering temperature $T_{\mathrm{CO}}$, where first $\mathrm{CO}$ clusters are formed, i.e. correlated polarons. Below $T_{\mathrm{CO}}$, a critical magnetic field of $H=20 \mathrm{kOe}$ breaks the AFM coupling between the ferromagnetic domains resulting in metamagnetic transitions and the onset of magnetic-field-induced intensity effects in the Raman spectra. Because of the high amount of correlated polarons, the narrow range where 
CMR and MI transition develops can be interpreted as a highly disordered, glassy state, similar to the short-range order in amorphous materials. This results in a broadening of the JT modes and their description by the phonon density-of-states. As a consequence, the observed Raman intensity behavior was discussed within the picture of a disorder-order transition. Furthermore, the significance of correlated polarons was underlined by the quantitative resemblance of the CMR $(\approx 7000 \%)$ and the magnetic-field-induced suppression $(\approx 6000 \%)$ of the JT stretching modes.

In addition, polaron formation at the manganite surface was revealed by means of surface-enhanced Raman spectroscopy. The presented SERS study constitutes the highlight of this cumulative thesis, since it represents the first of its kind. For this, the MAD technique was improved to grow metallic nanostructures to perform surface-enhanced Raman studies on correlated oxides. To determine the capabilities of the MAD-based SERS substrates, the well-known oxide $\mathrm{TiO}_{2}$ was chosen as a first model system (see $\mathrm{Ch} .5$ ). Within this thesis, successful growth of gold nanoparticles by the MAD was demonstrated, which allowed for the characterization of a $3 \mathrm{~nm}$ thin $\mathrm{TiO}_{2}$ film. Furthermore, a homogeneous enhancement with an averaged $E F \approx 10^{7}$ was realized. This novel approach permitted the noninvasive deposition of gold nanoparticles on the manganite surface. The continuous oxygen flow within the MAD chamber minimizes deoxygenation effects at the manganite surface and thereby, preventing an artificially created surface structure, which was validated by transport measurements on ultrathin films. The SERS study revealed a $\mathrm{Mn}^{3+}$-enriched and, thus, Jahn-Teller reconstructed surface layer. Such polaronic layer at the surface provides an additional electrostatic barrier and can be considered as a reasonable origin of the electrical and magnetic dead layer at a given interface. Taking into account the rapid decrease of the plasmonic enhancement, a thickness between 4 and 7 u.c. of the polaron enriched surface layer was estimated. This is in line with XMCD measurements of the $\mathrm{LSMO} / \mathrm{SrTiO}_{3}$ interface [41, 43, 103, 395], c-AFM measurements [187] and theoretical calculations by CALDERón [39]. The similar thicknesses of the insulating nonmagnetic layer further hints at a characteristic charge transfer length for the accommodation of the polar catastrophe at manganite interfaces. The importance, and possible generality, of such correlation length for manganite structures (and likely correlated materials in general) is further shown by the recent observation of a high- $T_{\mathrm{C}}$ phase and the observed charge injection length of about 2 u.c..

Therefore, this thesis did not only provide further insight into the mechanisms behind CMR and MI transition, it also provided evidence for polaron formation at the manganite surface. In this connection, a preferred polaron accumulation at the interface was revealed, emphasizing the significance of phase boundaries for fundamental and applied manganite physics. Certainly, this thesis could not address all details of the polaronic behavior within the disorder-order transition and at the manganite surface. Therefore, a prospect of further studies will be given in the following chapter. Note that results presented therein are preliminary and, thus, the 
interpretation is still under debate and may not represent the final argumentation.

\section{Temperature-dependent SERS Studies}

An interesting point would be to study the temperature-dependent behavior of the surface. Transport measurements on ultrathin LSMO films revealed a broad low-temperature magnetoresistance at $T=100-200 \mathrm{~K}$, which was attributed to the magnetic transition of the dead layer [28]. Such a transition should also be accompanied by a suppression of the polaronic surface and a corresponding reduction of the thickness of the distorted layer [103, 104]. Likely, this would become apparent by similar features as seen in the bulk spectra. In the final stages of this thesis, first attempts were made to perform temperature-dependent SERS measurements on a thin LSMO/ $\mathrm{LaAlO}_{3}(100)$ film. Two sets of temperature-dependent SER spectra were collected at two different positions on the film surface and are depicted in Fig.7.1. However, both data sets show a completely different temperature dependence. While the spectra in panel (a) clearly exhibit a similar increase and decrease of the JT modes, the intensity of the JT modes in the spectra in panel (b) solely increase with decreasing temperature. Therefore, the former one reveals a behavior similar to the one observed in bulk material (see $\mathrm{Ch} .3$ and 4), whereas the latter one exhibits phonon properties analogously to LMO [64. Such behavior may indicate an inhomogeneous surface structure consisting of regions, where a disorder-order transition similar to LPCMO occurs, dubbed as CE-type structure in the following, and in which a cooperative Jahn-Teller effect is formed similar to LMO. Furthermore, several new features in the mid-frequency region $\left(250-400 \mathrm{~cm}^{-1}\right)$ emerge at low temperatures. These modes coincide with Raman-active vibrations of the orthorhombic manganite structure [58, 59], supporting the suggested structural phase transition at the surface (see Ch.60). However, a closer look into the second set of temperature-dependent SER spectra indicates a relatively odd intensity behavior.

While cooling down from room temperature to $280 \mathrm{~K}$ induces a huge intensity increase, a further decrease of the temperature down to $210 \mathrm{~K}$ does not change the intensity level of the Raman modes further. This is unexpected for a cooperativelike LMO structure, since a further increase of the intensity of the JT modes should occur when reducing the temperature. Moreover, the continuing reduction of the temperature down to $120 \mathrm{~K}$ causes a huge intensity gain, but, also additional features arise, which could not be observed in this manner in the first set of temperaturedependent SER spectra. The spectral structure of the rotational modes around $200 \mathrm{~cm}^{-1}$ exhibits severe differences and an additional broad feature at higher frequencies is revealed $\left(800-1000 \mathrm{~cm}^{-1}\right)$. The broad high-frequency feature coincides with an electronic mode caused by the small polaron ionization [249], observed in mixed-valence manganites [249, 401]. However, such a spectral feature should also occur in the CE-type structure, since it is also present in the temperatureand magnetic-field-dependent Raman study shown in $\mathrm{Ch} .3$ and 4 . A more detailed temperature-dependent study of the polaronic behavior at the surface may clarify 

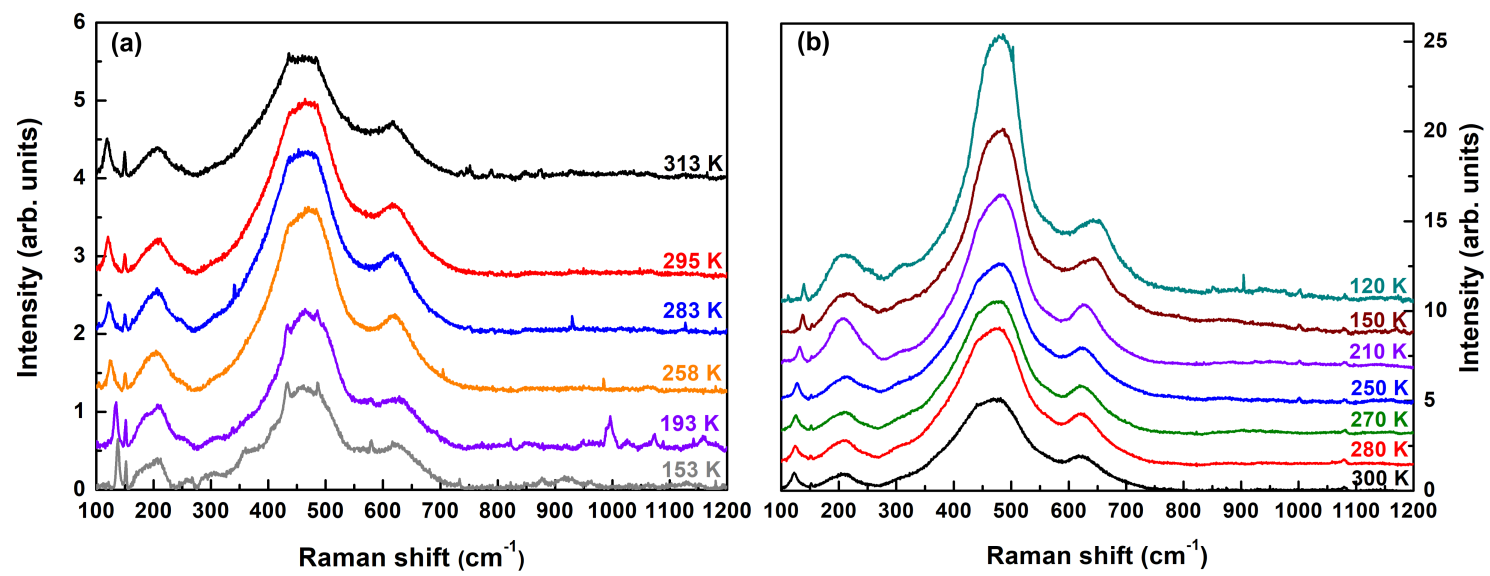

Figure 7.1.: Temperature-dependent SERS experiments of a LSMO/LAO(100) film $(d=60 \mathrm{~nm})$. Both studies, although performed on the same sample, exhibit a highly different intensity behavior. While the first one (a) exhibits a LPCMO-like disorder-order transition, indicated by the intensity of the JT modes, the second shows a monotonic increase, similar to the temperature-dependent Raman spectra of LMO. All SER spectra are normalized to the strongest Raman mode of the LAO substrate $\left(\omega=122 \mathrm{~cm}^{-1}\right)$.

this unusual temperature-dependent behavior at the surface and may give deeper insights into the behavior of the dead layer, thus, constituting interesting prospects for future studies.

\section{Surface Engineering}

Furthermore, first attempts to artificially engineer the surface are performed within the master thesis of Jonas Wawra, which was supervised by the author during the time this thesis was written. Within this master thesis, thin LCMO and LSMO films are capped with different oxide layers, such as $\mathrm{LaAlO}_{3}$ (LAO), $\mathrm{SrTiO}_{3}$ (STO), LMO and SMO, in order to study their influence on the JT distorted surface layer. Currently, the artificial tailoring of the dead layer exhibits a highly active research field, which was facilitated by recent advances in thin film deposition techniques, allowing the deposition of films only a monolayer thick. The idea behind such an endeavor is on the one hand to inject charge carriers at the interface, e.g. by inserting a LMO buffer layer at the film/substrate interface [409], and on the other hand to artificially tailor the octahedral rotations at the film/substrate interface [234, 410, 411]. Both processes should reduce the electrostatic barrier known as dead layer, which is crucial for possible applications of manganite films in electronic devices. SERS studies on such heterostructures could contribute to the discussion by shedding light on the impact of such capping/buffer layers onto the surface, as Raman scattering is able to monitor the polaronic behavior [65, 66, 268, 401] at the artificially created interface. Furthermore, it is sensitive to structural changes, e.g. 
octahedral tilting, due to the low-frequency rotational modes [58, 59, 259]. However, since the plasmonic enhancement scales with $1 / r^{3}$, the capping layer has to be only a few monolayers thick. In this thesis, a thickness of $1 \mathrm{~nm}(\approx 2$ u.c. $)$ was chosen, because it should ensure the growth of a closed film on top the surface, but also guarantees a sufficient enhancement to study the capping-layer-induced effects, as shown by preliminary performed distance-dependence studies (see Fig.7.2).

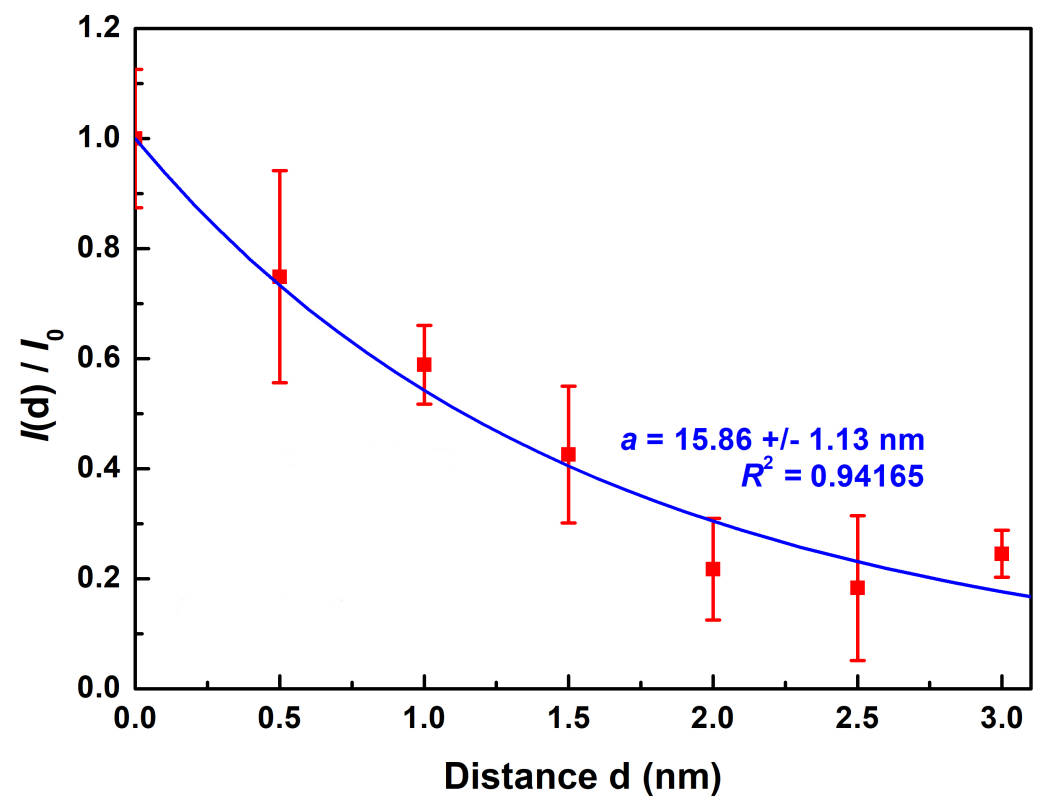

Figure 7.2.: Distance-dependent study of the plasmonic enhancement, performed on $\mathrm{Al}_{2} \mathrm{O}_{3} / \mathrm{LCMO}$ stacks. For a layer thickness of $1 \mathrm{~nm}(\approx 2$ u.c. $)$, the signal is decreased to $60 \%$, providing a sufficient enhancement for the study of interface effects due to the deposited capping layer. One further obtains a particle size of the enhancing particle, here $a_{A u} \approx 16 \mathrm{~nm}$ by means of fitting Eq.2.20 from Ch.2.3 (blue line). This corresponds well to the estimated particle size by means of the particle size distribution in $\mathrm{Ch} .6$.

First, the deposition of LAO and STO capping layers was performed. The choice of LAO and STO as capping layers was motivated by a recent XAS study [395]. This study revealed an enhanced amount of $\mathrm{Mn}^{2+}$ at the surface by depositing a STO capping layer onto the surface, but, almost no changes of the mixed-valence state for the LAO capping layer. In addition, these oxides may constitute prototypical examples for insulating barriers in tunnel magnetoresistive devices [29, 32, 404]. Both oxides can further be used to monitor possible contributions to the SER spectra due to the capping layer. This would be significantly important for the interpretation of SER spectra of films with a LMO capping layer. The JT stretching vibrations of LMO would overlap with the induced JT modes of the free surface and, thus, changes induced by the LMO layer could not be unambiguously identified. In the 
following, preliminary results of the SER spectra of the films with LAO and STO capping layers will be presented. The SER spectra of the different surfaces are shown in Fig.7.3. Note that an in-depth analyse of this data was not present at the point this thesis was written.
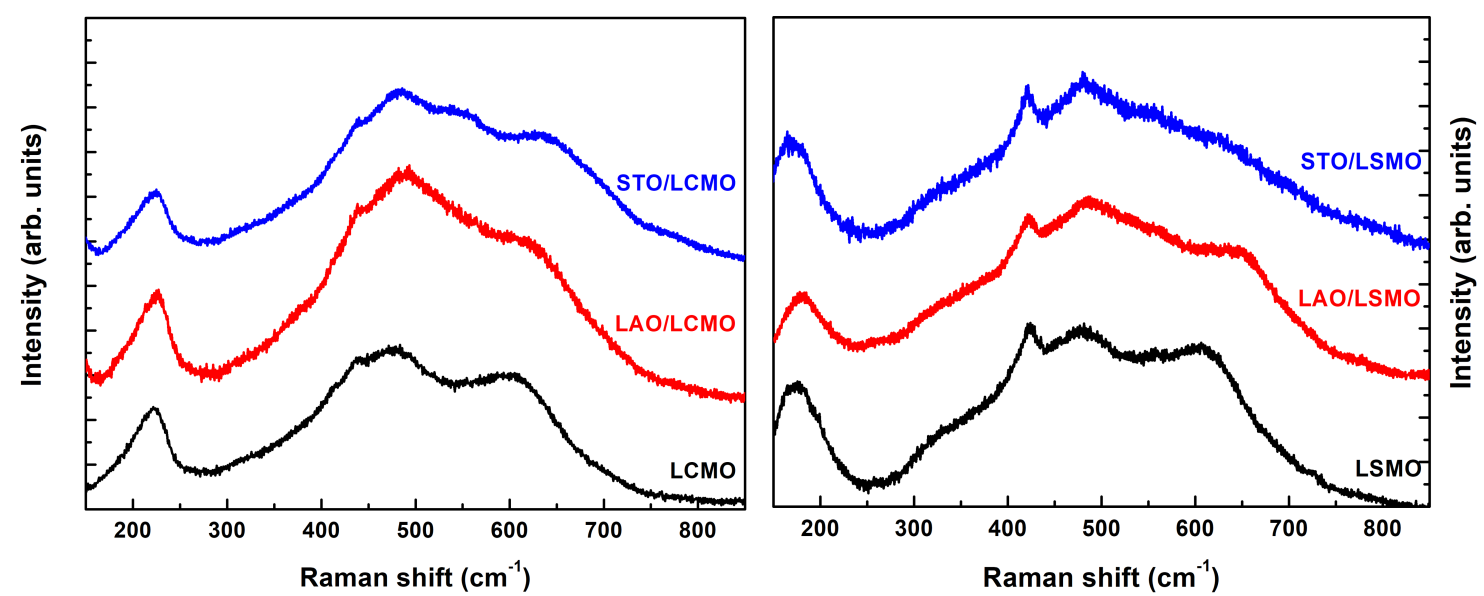

Figure 7.3.: SER spectra of the free manganite surface (black) of LCMO and LSMO compared to the SER spectra of the capped manganite surfaces (red: LAO; blue: STO).

At the first glance, the capping layers on LCMO induce a shift of the JT modes and a small suppression of the symmetric stretching vibration. The STO capping layer on LSMO, in turn, suppresses the JT mode and the spectrum resembles the bulk spectrum of LSMO. The LAO capping layer however induces rather structural changes, which are indicated by the shift of the JT modes. Overall, it seems that the capping layer reduces the intensity of the JT stretching vibrations and, thus, likely the JT distorted surface layer. The additional surface mode around $555 \mathrm{~cm}^{-1}$ further is pronounced, when growing a STO capping layer onto the manganite surface. In a subsequent step, the effect of charge and hole injection by means of LMO and SMO will be studied within the master thesis of Jonas Wawra. However, as aforementioned, the master thesis is still ongoing and no preliminary results concerning the LMO and SMO capping layers were obtained during the time this cumulative thesis was written. Furthermore, this study will provide a preliminary step to the facilitation of SERS on superlattices of correlated oxides, such as LMO/SMO superlattices, which could give further insight into the interfacial charge transfer mentioned.

In conclusion, the presented research contributes to the understanding of manganites, emphasizing the significance of (internal) microscopic and macroscopic interfaces in fundamental as well as applied manganite physics. It further opens the field of correlated oxides to an unique technique to study the interesting phenomena occurring at their surfaces and interfaces. 


\section{Bibliography}

[1] E. Dagotto. Complexity in Strongly Correlated Electronic Systems. Science, 309(5732):257-262, 2005.

[2] H. Keller, A. Bussmann-Holder, and K. A. Müller. Jahn-Teller physics and high- $T_{\mathrm{C}}$ superconductivity. Materials Today, 11(9):38-46, 2008.

[3] H. Hosono, A. Yamamoto, H. Hiramatsu, and Y. Ma. Recent advances in ironbased superconductors toward applications. Materials Today, 21(3):278-302, 2018.

[4] G. H. Jonker and J. H. Van Santen. Ferromagnetic compounds of manganese with perovskite structure. Physica, 16(3):337-349, 1950.

[5] V. Pfahl, M. K. Phani, M. Büchsenschütz-Göbeler, A. Kumar, V. Moshnyaga, W. Arnold, and K. Samwer. Conduction electrons as dissipation channel in friction experiments at the metal-metal transition of LSMO measured by contact-resonance atomic force microscopy. Applied Physics Letters, 110(5):053102, 2017.

[6] V. Pfahl, C. Ma, W. Arnold, and K. Samwer. Universal aspects of sonolubrication in amorphous and crystalline materials. Journal of Applied Physics, 123(3):035301, 2018.

[7] S. Raabe, D. Mierwaldt, J. Ciston, M. Uijttewaal, H. Stein, J. Hoffmann, Y. Zhu, P. Blöchl, and C. Jooss. In Situ Electrochemical Electron Microscopy Study of Oxygen Evolution Activity of Doped Manganite Perovskites. Advanced Functional Materials, 22(16):3378-3388, 2012.

[8] M. E. Abrishami, M. Risch, J. Scholz, V. Roddatis, N. Osterthun, and C. Jooss. Oxygen Evolution at Manganite Perovskite Ruddlesden-Popper Type Particles: Trends of Activity on Structure, Valence and Covalence. Materials (Basel, Switzerland), 9(11), 2016.

[9] T. Mertelj, D. Kuščer, M. Kosec, and D. Mihailovic. Photoinduced infrared absorption in $\mathrm{La}_{1-x} \mathrm{Sr}_{x} \mathrm{Mn}_{1-\delta} \mathrm{O}_{3}$ : Changes of the small polaron binding energy with doping. Physical Review B, 61(22):15102-15107, 2000. 
[10] M. Mchalwat, H. Ulrichs, J. Walowski, C. Ballani, O. Shapoval, V. Moshnyaga, M. Münzenberg, and K. Samwer. Laser-induced changes of nonlinear electronic transport properties in $\mathrm{La}_{0.75} \mathrm{Ba}_{0.25} \mathrm{MnO}_{3}$ and $\left(\mathrm{La}_{0.6} \mathrm{Pr}_{0.4}\right)_{0.7} \mathrm{Ca}_{0.3} \mathrm{MnO}_{3}$. Journal of Physics: Condensed Matter, 30(4):045701, 2018.

[11] E. Kamil, R. Schade, T. Pruschke, and P. E. Blöchl. Reduced density-matrix functionals applied to the Hubbard dimer. Physical Review B, 93(8), 2016.

[12] R. Schade, E. Kamil, and P. Blöchl. Reduced density-matrix functionals from many-particle theory. The European Physical Journal Special Topics, 226(11):2677-2692, 2017.

[13] R. Schade and P. E. Blöchl. Adaptive cluster approximation for reduced density-matrix functional theory. Physical Review B, 97(24), 2018.

[14] T. Köhler, S. Rajpurohit, O. Schumann, S. Paeckel, F. R. A. Biebl, M. Sotoudeh, S. C. Kramer, P. E. Blöchl, S. Kehrein, and S. R. Manmana. Relaxation of photoexcitations in polaron-induced magnetic microstructures. Physical Review B, 97(23), 2018.

[15] J. H. Ngai, F. J. Walker, and C. H. Ahn. Correlated Oxide Physics and Electronics. Annual Review of Materials Research, 44(1):1-17, 2014.

[16] S.-W. Cheong and M. Mostovoy. Multiferroics: a magnetic twist for ferroelectricity. Nature Materials, 6(1):13-20, 2007.

[17] H. A. Jahn and E. Teller. Stability of polyatomic molecules in degenerate electronic states - I. Orbital degeneracy. Proceedings of the Royal Society of London. Series A - Mathematical and Physical Sciences, 161(905):220-235, 1937.

[18] H. Nickisch, H. Thomas, and K.-H. Höck. Jahn-Teller effect in ititnerant electron systems: the Jahn-Teller polaron. Helvetica Physica Acta, 56(1-3):237, 1983.

[19] J. G. Bednorz and K. A. Müller. Possible high- $T_{\mathrm{C}}$ superconductivity in the BaLa-Cu-O system. Zeitschrift für Physik B Condensed Matter, 64(2):189-193, 1986.

[20] V. Moshnyaga and K. Samwer. Electron-lattice correlations and phase transitions in CMR manganites. Annalen der Physik, 523(8-9):652-663, 2011.

[21] K.-I. Chahara, T. Ohno, M. Kasai, and Y. Kozono. Magnetoresistance in magnetic manganese oxide with intrinsic antiferromagnetic spin structure. Applied Physics Letters, 63(14):1990-1992, 1993. 
[22] R. von Helmolt, J. Wecker, B. Holzapfel, L. Schultz, and K. Samwer. Giant negative magnetoresistance in perovskitelike $\mathrm{La}_{0.7} \mathrm{Ba}_{0.3} \mathrm{MnO}_{3}$ ferromagnetic films. Physical Review Letters, 71(14):2331-2333, 1993.

[23] A. Urushibara, Y. Moritomo, T. Arima, A. Asamitsu, G. Kido, and Y. Tokura. Insulator-metal transition and giant magnetoresistance in $\mathrm{La}_{1-x} \mathrm{Sr}_{x} \mathrm{MnO}_{3}$. Physical Review B, 51(20):14103-14109, 1995.

[24] Z. Liao and J. Zhang. Metal-to-Insulator Transition in Ultrathin Manganite Heterostructures. Applied Sciences, 9(1):144, 2019.

[25] A. P. Ramirez. Colossal magnetoresistance. Journal of Physics: Condensed Matter, 9(39):8171-8199, 1997.

[26] Y. Tokura and Y. Tomioka. Colossal magnetoresistive manganites. Journal of Magnetism and Magnetic Materials, 200(1-3):1-23, 1999.

[27] H. Y. Hwang, S.-W. Cheong, N. P. Ong, and B. Batlogg. Spin-Polarized Intergrain Tunneling in $\mathrm{La}_{0.67} \mathrm{Sr}_{0.33} \mathrm{MnO}_{3}$. Physical Review Letters, 77(10):20412044, 1996.

[28] J. Z. Sun, D. W. Abraham, R. A. Rao, and C. B. Eom. Thickness-dependent magnetotransport in ultrathin manganite films. Applied Physics Letters, 74(20):3017-3019, 1999.

[29] D. C. Worledge and T. H. Geballe. Spin-polarized tunneling in $\mathrm{La}_{0.67} \mathrm{Sr}_{0.33} \mathrm{MnO}_{3}$. Applied Physics Letters, 76(7):900-902, 2000.

[30] J. O’Donnell, A. E. Andrus, S. Oh, E. V. Colla, and J. N. Eckstein. Colossal magnetoresistance magnetic tunnel junctions grown by molecular-beam epitaxy. Applied Physics Letters, 76(14):1914-1916, 2000.

[31] R. P. Borges, W. Guichard, J. G. Lunney, J. M. D. Coey, and F. Ott. Magnetic and electric "dead" layers in $\mathrm{La}_{0.7} \mathrm{Sr}_{0.3} \mathrm{MnO}_{3}$ thin films. Journal of Applied Physics, 89(7):3868-3873, 2001.

[32] J. Simon, T. Walther, W. Mader, J. Klein, D. Reisinger, L. Alff, and R. Gross. Diffusion and segregation effects in doped manganite/titanate heterostructures. Applied Physics Letters, 84(19):3882-3884, 2004.

[33] N. Nakagawa, H. Y. Hwang, and D. A. Muller. Why some interfaces cannot be sharp. Nature Materials, 5(3):204-209, 2006.

[34] Seungbum Hong, Serge M. Nakhmanson, and Dillon D. Fong. Screening mechanisms at polar oxide heterointerfaces. Reports on Progress in Physics, 79(7):076501, 2016. 
[35] D. Yi, N. Lu, X. Chen, S. Shen, and P. Yu. Engineering magnetism at functional oxides interfaces: manganites and beyond. Journal of Physics: Condensed Matter, 29(44):443004, 2017.

[36] H. Dulli, E. W. Plummer, P. A. Dowben, Jaewu Choi, and S.-H. Liou. Surface electronic phase transition in colossal magnetoresistive manganese perovskites: $\mathrm{La}_{0.65} \mathrm{Sr}_{0.35} \mathrm{MnO}_{3}$. Applied Physics Letters, 77(4):570-572, 2000.

[37] H. Dulli, P. A. Dowben, S.-H. Liou, and E. W. Plummer. Surface segregation and restructuring of colossal-magnetoresistant manganese perovskite $\mathrm{La}_{0.65} \mathrm{Sr}_{0.35} \mathrm{MnO}_{3}$. Physical Review B, 62(22):R14629-R14632, 2000.

[38] C. N. Borca, Delia Ristoiu, Q. L. Xu, S.-H. Liou, S. Adenwalla, and P. A. Dowben. The surface terminal layer and composition of the colossal magnetoresistance perovskite: $\mathrm{La}_{0.65} \mathrm{~Pb}_{0.35} \mathrm{MnO}_{3}$. Journal of Applied Physics, 87(9):61046106, 2000.

[39] M. J. Calderón, L. Brey, and F. Guinea. Surface electronic structure and magnetic properties of doped manganites. Physical Review B, 60(9):66986704, 1999.

[40] H. Zenia, G. A. Gehring, G. Banach, and W. M. Temmerman. Electronic and magnetic properties of the (001) surface of hole-doped manganites. Physical Review B, 71(2):R14, 2005.

[41] J.-S. Lee, D. A. Arena, P. Yu, C. S. Nelson, R. Fan, C. J. Kinane, S. Langridge, M. D. Rossell, R. Ramesh, and C.-C. Kao. Hidden Magnetic Configuration in Epitaxial La ${ }_{1-x} \mathrm{Sr}_{x} \mathrm{MnO}_{3}$ Films. Physical Review Letters, 105(25), 2010.

[42] R. Peng, H. C. Xu, M. Xia, J. F. Zhao, X. Xie, D. F. Xu, B. P. Xie, and D. L. Feng. Tuning the dead-layer behavior of $\mathrm{La}_{0.67} \mathrm{Sr}_{0.33} \mathrm{MnO}_{3} / \mathrm{SrTiO}_{3}$ via interfacial engineering. Applied Physics Letters, 104(8):081606, 2014.

[43] J. A. Mundy, Y. Hikita, T. Hidaka, T. Yajima, T. Higuchi, H. Y. Hwang, D. A. Muller, and L. F. Kourkoutis. Visualizing the interfacial evolution from charge compensation to metallic screening across the manganite metal-insulator transition. Nature Communications, 5:3464, 2014.

[44] Zuhuang Chen, Zhanghui Chen, Z. Q. Liu, M. E. Holtz, C. J. Li, X. Renshaw Wang, W. M. Lü, M. Motapothula, L.S. Fan, J. A. Turcaud, L. R. Dedon, C. Frederick, R. J. Xu, R. Gao, A.T. N'Diaye, E. Arenholz, J. A. Mundy, T. Venkatesan, D. A. Muller, L.-W. Wang, Jian Liu, and L. W. Martin. Electron Accumulation and Emergent Magnetism in $\mathrm{LaMnO}_{3} / \mathrm{SrTiO}_{3}$ Heterostructures. Physical Review Letters, 119(15), 2017.

[45] J. Garcia-Barriocanal, J. C. Cezar, F. Y. Bruno, P. Thakur, N. B. Brookes, C. Utfeld, A. Rivera-Calzada, S. R. Giblin, J. W. Taylor, J. A. Duffy, S. B. 
Dugdale, T. Nakamura, K. Kodama, C. Leon, S. Okamoto, and J. Santamaria. Spin and orbital Ti magnetism at $\mathrm{LaMnO}_{3} / \mathrm{SrTiO}_{3}$ interfaces. Nature Communications, 1(1), 2010.

[46] M.-B. Lepetit, B. Mercey, and C. Simon. Interface Effects in Perovskite Thin Films. Physical Review Letters, 108(8), 2012.

[47] R. Loudon. The Raman effect in crystals. Advances in Physics, 13(52):423482, 1964.

[48] K. Kneipp, H. Kneipp, I. Itzkan, R. R. Dasari, and M. S. Feld. Surfaceenhanced Raman scattering and biophysics. Journal of Physics: Condensed Matter, 14(18):R597-R624, 2002.

[49] R. Shuker and R. W. Gammon. Raman-Scattering Selection-Rule Breaking and the Density of States in Amorphous Materials. Physical Review Letters, 25(4):222-225, 1970.

[50] P. Colomban, A. Tournie, and L. Bellot-Gurlet. Raman identification of glassy silicates used in ceramics, glass and jewellery: a tentative differentiation guide. Journal of Raman Spectroscopy, 37(8):841-852, 2006.

[51] G. Gouadec and P. Colomban. Raman Spectroscopy of nanomaterials: How spectra relate to disorder, particle size and mechanical properties. Progress in Crystal Growth and Characterization of Materials, 53(1):1-56, 2007.

[52] W. D. W. Abney and L. C. Festing. XX. On the influence of the atomic grouping in the molecules of organic bodies on their absorption in the infrared region of the spectrum. Philosophical Transactions of the Royal Society of London, 172:887-918, 1881.

[53] C. V. Raman and K. S. Krishnan. A New Type of Secondary Radiation. Nature, 121(3048):501-502, 1928.

[54] G. Landsberg and L. Mandelstam. Eine neue Erscheinung bei der Lichtzerstreuung in Krystallen. Die Naturwissenschaften, 16(28):557-558, 1928.

[55] L. Andrew Lyon, Christine D. Keating, Audrey P. Fox, Bonnie E. Baker, Lin He, Sheila R. Nicewarner, Shawn P. Mulvaney, and Michael J. Natan. Raman Spectroscopy. Analytical Chemistry, 70(12):341-362, 1998.

[56] J. R. Baena and B. Lendl. Raman spectroscopy in chemical bioanalysis. Current Opinion in Chemical Biology, 8(5):534-539, 2004.

[57] T. P. Devereaux and R. Hackl. Inelastic light scattering from correlated electrons. Reviews of Modern Physics, 79(1):175-233, 2007. 
[58] M. N. Iliev, M. V. Abrashev, H.-G. Lee, V. N. Popov, Y. Y. Sun, C. Thomsen, R. L. Meng, and C. W. Chu. Raman spectroscopy of orthorhombic perovskitelike $\mathrm{YMnO}_{3}$ andLaMnO 3 . Physical Review B, 57(5):2872-2877, 1998.

[59] M. N. Iliev, M. V. Abrashev, J. Laverdière, S. Jandl, M. M. Gospodinov, Y.-Q. Wang, and Y.-Y. Sun. Distortion-dependent Raman spectra and mode mixing in $\mathrm{RMnO}_{3}$ perovskites ( $\left.\mathrm{R}=\mathrm{La}, \mathrm{Pr}, \mathrm{Nd}, \mathrm{Sm}, \mathrm{Eu}, \mathrm{Gd}, \mathrm{Tb}, \mathrm{Dy}, \mathrm{Ho}, \mathrm{Y}\right)$. Physical Review B, 73(6):413, 2006.

[60] M. Kim, H. Barath, S. L. Cooper, P. Abbamonte, E. Fradkin, M. Rübhausen, C. L. Zhang, and S.-W. Cheong. Raman scattering studies of the temperatureand field-induced melting of charge order in $\mathrm{La}_{x} \mathrm{Pr}_{y} \mathrm{Ca}_{1-x-y} \mathrm{MnO}_{3}$. Physical Review B, 77(13):8171, 2008.

[61] M. V. Abrashev, V. G. Avanov, M. N. Iliev, R. A. Chakalov, R. I. Chakalova, and C. Thomsen. Raman Study of the Variations of the Jahn-Teller Distortions through the Metal-Insulator Transition in Magnetoresistive $\mathrm{La}_{0.7} \mathrm{Ca}_{0.3} \mathrm{MnO}_{3}$ Thin Films. physica status solidi (b), 215(1):631-636, 1999.

[62] E. Granado, N. O. Moreno, A. García, J. A. Sanjurjo, C. Rettori, I. Torriani, S. B. Oseroff, J. J. Neumeier, K. J. McClellan, S.-W. Cheong, and Y. Tokura. Phonon Raman scattering in $\mathrm{R}_{1-x} \mathrm{~A}_{x} \mathrm{MnO}_{3+\delta}(\mathrm{R}=\mathrm{La}, \mathrm{Pr} \quad \mathrm{A}=\mathrm{Ca}, \mathrm{Sr})$. Physical Review B, 58(17):11435-11440, 1998.

[63] E. Granado, A. García, J. A. Sanjurjo, C. Rettori, I. Torriani, F. Prado, R. D. Sánchez, A. Caneiro, and S. B. Oseroff. Magnetic ordering effects in the Raman spectra of $\mathrm{La}_{1-x} \mathrm{~A}_{x} \mathrm{MnO}_{3}$. Physical Review B, 60(17):11879-11882, 1999.

[64] E. Granado, J. A. Sanjurjo, C. Rettori, J. J. Neumeier, and S. B. Oseroff. Order-disorder in the Jahn-Teller transition of $\mathrm{LaMnO}_{3}$ : A Raman scattering study. Physical Review B, 62(17):11304-11307, 2000.

[65] M. N. Iliev, M. V. Abrashev, V. N. Popov, and V. G. Hadjiev. Role of JahnTeller disorder in Raman scattering of mixed-valence manganites. Physical Review B, 67(21):4393, 2003.

[66] V. Moshnyaga, A. Belenchuk, S. Hühn, C. Kalkert, M. Jungbauer, O. I. Lebedev, S. Merten, K.-Y. Choi, P. Lemmens, B. Damaschke, and K. Samwer. Intrinsic antiferromagnetic coupling underlies colossal magnetoresistance effect: Role of correlated polarons. Physical Review B, 89(2), 2014.

[67] A. Sacchetti, P. Dore, P. Postorino, and A. Congeduti. Pressure and temperature dependence of optical phonons in $\mathrm{La}_{0.75} \mathrm{Sr}_{0.25} \mathrm{MnO}_{3}$. Journal of Physics and Chemistry of Solids, 65(8-9):1431-1437, 2004.

[68] M. Moskovits. Surface-enhanced spectroscopy. Reviews of Modern Physics, $57(3): 783-826,1985$. 
[69] E. Bailo and V. Deckert. Tip-enhanced Raman scattering. Chemical Society Reviews, 37(5):921-930, 2008.

[70] X. Wang, S.-C. Huang, T.-X. Huang, H.-S. Su, J.-H. Zhong, Z.-C. Zeng, M.-H. $\mathrm{Li}$, and B. Ren. Tip-enhanced Raman spectroscopy for surfaces and interfaces. Chemical Society Reviews, 46(13):4020-4041, 2017.

[71] E. C. Le Ru and P. G. Etchegoin. Rigorous justification of the $|\mathrm{E}|^{4}$ enhancement factor in Surface Enhanced Raman Spectroscopy. Chemical Physics Letters, 423(1-3):63-66, 2006.

[72] S. L. McCall, P. M. Platzman, and P. A. Wolff. Surface enhanced Raman scattering. Physics Letters A, 77(5):381-383, 1980.

[73] B. J. Kennedy, S. Spaeth, M. Dickey, and K. T. Carron. Determination of the Distance Dependence and Experimental Effects for Modified SERS Substrates Based on Self-Assembled Monolayers Formed Using Alkanethiols. The Journal of Physical Chemistry B, 103(18):3640-3646, 1999.

[74] S. S. Masango, R. A. Hackler, N. Large, A.-I. Henry, M. O. McAnally, G. C. Schatz, P. C. Stair, and R. P. van Duyne. High-Resolution Distance Dependence Study of Surface-Enhanced Raman Scattering Enabled by Atomic Layer Deposition. Nano Letters, 16(7):4251-4259, 2016.

[75] K. Kneipp, Y. Wang, H. Kneipp, L. T. Perelman, I. Itzkan, R. R. Dasari, and M. S. Feld. Single Molecule Detection Using Surface-Enhanced Raman Scattering (SERS). Physical Review Letters, 78(9):1667-1670, 1997.

[76] K. Kneipp, H. Kneipp, I. Itzkan, R. R. Dasari, and M. S. Feld. Ultrasensitive Chemical Analysis by Raman Spectroscopy. Chemical Reviews, 99(10):29572976, 1999.

[77] J. Jiang, K. Bosnick, M. Maillard, and L. Brus. Single Molecule Raman Spectroscopy at the Junctions of Large Ag Nanocrystals. The Journal of Physical Chemistry B, 107(37):9964-9972, 2003.

[78] E. C. Le Ru, E. Blackie, M. Meyer, and P. G. Etchegoin. Surface Enhanced Raman Scattering Enhancement Factors: A Comprehensive Study. The Journal of Physical Chemistry C, 111(37):13794-13803, 2007.

[79] S. Pahlow, A. März, B. Seise, K. Hartmann, I. Freitag, E. Kämmer, R. Böhme, V. Deckert, K. Weber, D. Cialla, and J. Popp. Bioanalytical application of surface- and tip-enhanced Raman spectroscopy. Engineering in Life Sciences, 12(2):131-143, 2012. 
[80] J. Cailletaud, C. de Bleye, E. Dumont, P.-Y. Sacré, L. Netchacovitch, Y. Gut, M. Boiret, Y.-M. Ginot, Ph Hubert, and E. Ziemons. Critical review of surfaceenhanced Raman spectroscopy applications in the pharmaceutical field. Journal of Pharmaceutical and Biomedical Analysis, 147:458-472, 2018.

[81] S. Stöckel, J. Kirchhoff, U. Neugebauer, P. Rösch, and J. Popp. The application of Raman spectroscopy for the detection and identification of microorganisms. Journal of Raman Spectroscopy, 47(1):89-109, 2016.

[82] S. Degioanni, A.-M. Jurdyc, F. Bessueille, J. Coulm, B. Champagnon, and D. Vouagner. Surface-enhanced Raman scattering of amorphous $\mathrm{TiO}_{2}$ thin films by gold nanostructures: Revealing first layer effect with thickness variation. Journal of Applied Physics, 114(23):234307, 2013.

[83] C.-T. Ko, P.-S. Yang, Y.-Y. Han, W.-C. Wang, J.-J. Huang, Y.-H. Lee, Y.J. Tsai, J. Shieh, and M.-J. Chen. Atomic-layer-deposited silver and dielectric nanostructures for plasmonic enhancement of Raman scattering from nanoscale ultrathin films. Nanotechnology, 26(26):265702, 2015.

[84] C. Meyer, S. Hühn, M. Jungbauer, S. Merten, B. Damaschke, K. Samwer, and V. Moshnyaga. Tip-enhanced Raman spectroscopy (TERS) on double perovskite $\mathrm{La}_{2} \mathrm{CoMnO}_{6}$ thin films: field enhancement and depolarization effects. Journal of Raman Spectroscopy, 48(1):46-52, 2017.

[85] M. Rajeswari, R. Shreekala, A. Goyal, S. E. Lofland, S. M. Bhagat, K. Ghosh, R. P. Sharma, R. L. Greene, R. Ramesh, T. Venkatesan, and T. Boettcher. Correlation between magnetic homogeneity, oxygen content, and electrical and magnetic properties of perovskite manganite thin films. Applied Physics Letters, 73(18):2672-2674, 1998.

[86] J. Dho, N. H. Hur, I. S. Kim, and Y. K. Park. Oxygen pressure and thickness dependent lattice strain in $\mathrm{La}_{0.7} \mathrm{Sr}_{0.3} \mathrm{MnO}_{3}$ films. Journal of Applied Physics, 94(12):7670, 2003.

[87] M. P. de Jong, V. A. Dediu, C. Taliani, and W. R. Salaneck. Electronic structure of $\mathrm{La}_{0.7} \mathrm{Sr}_{0.3} \mathrm{MnO}_{3}$ thin films for hybrid organic/inorganic spintronics applications. Journal of Applied Physics, 94(11):7292-7296, 2003.

[88] L. Feigl, B. D. Schultz, S. Ohya, D. G. Ouellette, A. Kozhanov, and C. J. Palmstrøm. Structural and transport properties of epitaxial $\mathrm{PrNiO}_{3}$ thin films grown by molecular beam epitaxy. Journal of Crystal Growth, 366:51-54, 2013.

[89] C. N. Borca, B. Xu, T. Komesu, H.-K. Jeong, M. T. Liu, S.-H. Liou, S. Stadler, Y. Idzerda, and P. A. Dowben. Electronic-structure modifications induced by surface segregation in $\mathrm{La}_{0.65} \mathrm{~Pb}_{0.35} \mathrm{MnO}_{3}$ thin films. Europhysics Letters (EPL), 56(5):722-728, 2001. 
[90] M. P. de Jong, I. Bergenti, V. A. Dediu, M. Fahlman, M. Marsi, and C. Taliani. Evidence for $\mathrm{Mn}^{2+}$ ions at surfaces of $\mathrm{La}_{0.7} \mathrm{Sr}_{0.3} \mathrm{MnO}_{3}$ thin films. Physical Review B, 71(1):R14, 2005.

[91] M. P. de Jong, I. Bergenti, W. Osikowicz, R. Friedlein, V. A. Dediu, C. Taliani, and W. R. Salaneck. Valence electronic states related to $\mathrm{Mn}^{2+}$ at $\mathrm{La}_{0.7} \mathrm{Sr}_{0.3} \mathrm{MnO}_{3}$ surfaces characterized by resonant photoemission. Physical Review B, 73(5):1171, 2006.

[92] S. Valencia, A. Gaupp, W. Gudat, Ll. Abad, Ll. Balcells, A. Cavallaro, B. Martínez, and F. J. Palomares. Mn valence instability in $\mathrm{La}_{0.67} \mathrm{Ca}_{0.33} \mathrm{MnO}_{3}$ thin films. Physical Review B, 73(10):449, 2006.

[93] S. Valencia, A. Gaupp, W. Gudat, Ll. Abad, Ll. Balcells, and B. Martínez. Impact of microstructure on the $\mathrm{Mn}$ valence of $\mathrm{La}_{0.67} \mathrm{Ca}_{0.33} \mathrm{MnO}_{3}$ thin films. Physical Review B, 75(18):449, 2007.

[94] S. Brivio, M. Cantoni, D. Petti, A. Cattoni, R. Bertacco, M. Finazzi, F. Ciccacci, A. Sidorenko, G. Allodi, M. Ghidini, and R. de Renzi. Decrease of the Curie temperature in $\mathrm{La}_{0.67} \mathrm{Sr}_{0.33} \mathrm{MnO}_{3}$ thin films induced by Au capping. Materials Science and Engineering: B, 144(1-3):93-96, 2007.

[95] R. Bertacco, S. Brivio, M. Cantoni, A. Cattoni, D. Petti, M. Finazzi, F. Ciccacci, A. A. Sidorenko, M. Ghidini, G. Allodi, and R. de Renzi. Proximity effects induced by a gold layer on $\mathrm{La}_{0.67} \mathrm{Sr}_{0.33} \mathrm{MnO}_{3}$ thin films. Applied Physics Letters, 91(10):102506, 2007.

[96] S. Brivio, C. Magen, A. A. Sidorenko, D. Petti, M. Cantoni, M. Finazzi, F. Ciccacci, R. de Renzi, M. Varela, S. Picozzi, and R. Bertacco. Effects of Au nanoparticles on the magnetic and transport properties of $\mathrm{La}_{0.67} \mathrm{Sr}_{0.33} \mathrm{MnO}_{3}$ ultrathin layers. Physical Review B, 81(9):150, 2010.

[97] N. Homonnay, K. J. ÓShea, C. Eisenschmidt, M. Wahler, D. A. MacLaren, and G. Schmidt. Interface Reactions in LSMO-Metal Hybrid Structures. ACS Applied Materials \& Interfaces, 7(40):22196-22202, 2015.

[98] V. Moshnyaga, I. Khoroshun, A. Sidorenko, P. Petrenko, A. Weidinger, M. Zeitler, B. Rauschenbach, R. Tidecks, and K. Samwer. Preparation of rareearth manganite-oxide thin films by metalorganic aerosol deposition technique. Applied Physics Letters, 74(19):2842-2844, 1999.

[99] E. Gommert, H. Cerva, J. Wecker, and K. Samwer. Influence of misfit stress on the magnetoresistive properties of $\mathrm{La}_{0.7} \mathrm{Ca}_{0.3} \mathrm{MnO}_{3-\delta}$ thin films. Journal of Applied Physics, 85(8):5417-5419, 1999.

[100] M. Jungbauer, S. Hühn, R. Egoavil, H. Tan, J. Verbeeck, G. van Tendeloo, and V. Moshnyaga. Atomic layer epitaxy of Ruddlesden-Popper $\mathrm{SrO}\left(\mathrm{SrTiO}_{3}\right)_{n}$ 
films by means of metalorganic aerosol deposition. Applied Physics Letters, 105(25):251603, 2014.

[101] M. Keunecke, F. Lyzwa, D. Schwarzbach, V. Roddatis, N. Gauquelin, K. Müller-Caspary, J. Verbeeck, S. J. Callori, F. Klose, M. Jungbauer, and V. Moshnyaga. High- $T_{\mathrm{C}}$ Interfacial Ferromagnetism in $\mathrm{SrMnO}_{3} / \mathrm{LaMnO}_{3} \mathrm{Su}-$ perlattices. Advanced Functional Materials, 11:1808270, 2019.

[102] J.-H. Park, E. Vescovo, H.-J. Kim, C. Kwon, R. Ramesh, and T. Venkatesan. Magnetic Properties at Surface Boundary of a Half-Metallic Ferromanget $\mathrm{La}_{0.67} \mathrm{Sr}_{0.33} \mathrm{MnO}_{3}$. Physical Review Letters, 81(9):1953-1956, 1998.

[103] J. W. Freeland, J. J. Kavich, K. E. Gray, L. Ozyuzer, H. Zheng, J. F. Mitchell, M. P. Warusawithana, P. Ryan, X. Zhai, R. H. Kodama, and J. N. Eckstein. Suppressed magnetization at the surfaces and interfaces of ferromagnetic metallic manganites. Journal of Physics: Condensed Matter, 19(31):315210, 2007.

[104] A. Verna, Bruce A. Davidson, Y. Szeto, A. Yu. Petrov, A. Mirone, A. Giglia, N. Mahne, and S. Nannarone. Measuring magnetic profiles at manganite surfaces with monolayer resolution. Journal of Magnetism and Magnetic Materials, 322(9-12):1212-1216, 2010.

[105] V. M. Goldschmidt. Die Gesetze der Krystallochemie. Die Naturwissenschaften, 14(21):477-485, 1926.

[106] H. Y. Hwang, S.-W. Cheong, P. G. Radaelli, M. Marezio, and B. Batlogg. Lattice Effects on the Magnetoresistance in Doped $\mathrm{LaMnO}_{3}$. Physical Review Letters, 75(5):914-917, 1995.

[107] K. Momma and F. Izumi. VESTA for three-dimensional visualization of crystal, volumetric and morphology data. Journal of Applied Crystallography, 44(6):1272-1276, 2011.

[108] J. B. Goodenough. Theory of the Role of Covalence in the Perovskite-Type Manganites[La, M(II)] $\mathrm{MnO}_{3}$. Physical Review, 100(2):564-573, 1955.

[109] A. J. Millis, Boris I. Shraiman, and R. Mueller. Dynamic Jahn-Teller Effect and Colossal Magnetoresistance in $\mathrm{La}_{1-x} \mathrm{Sr}_{x} \mathrm{MnO}_{3}$. Physical Review Letters, 77(1):175-178, 1996.

[110] J. M. D. Coey, M. Viret, and S. von Molnár. Mixed-valence manganites. Advances in Physics, 48(2):167-293, 1999.

[111] P. W. Anderson. Antiferromagnetism. Theory of Superexchange Interaction. Physical Review, 79(2):350-356, 1950. 
[112] J. Kanamori. Superexchange interaction and symmetry properties of electron orbitals. Journal of Physics and Chemistry of Solids, 10(2-3):87-98, 1959.

[113] M. Opel. Vorlesungsskript Magnetismus, Kapitel 6: Die Austauschwechselwirkung. http://www.wmi.badw.de/teaching/Lecturenotes/, 2005. Last called: 16.09.2019, 7.18 p.m.

[114] R. Gross. Vorlesungsskript Spinelektronik, Kapitel 4: Der Kolossale Magnetowiderstandseffekt. http://www.wmi.badw.de/teaching/Lecturenotes/, 2005. Last called: $16.09 .2019,7.18$ p.m.

[115] N. N. Kovaleva, Andrzej M. Oleś, A. M. Balbashov, A. Maljuk, D. N. Argyriou, G. Khaliullin, and B. Keimer. Low-energy Mott-Hubbard excitations in $\mathrm{LaMnO}_{3}$ probed by optical ellipsometry. Physical Review B, 81(23), 2010.

[116] A. Chainani, M. Mathew, and D. D. Sarma. Electron spectroscopic investigation of the semiconductor-metal transition in $\mathrm{La}_{1-x} \mathrm{Sr}_{x} \mathrm{MnO}_{3}$. Physical Review B, 47(23):15397-15403, 1993.

[117] J.-H. Park, C. T. Chen, S.-W. Cheong, W. Bao, G. Meigs, V. Chakarian, and Y. U. Idzerda. Electronic Aspects of the Ferromagnetic Transition in Manganese Perovskites. Physical Review Letters, 76(22):4215-4218, 1996.

[118] Y. Tokura. Orbital Physics in Transition-Metal Oxides. Science, 288(5465):462-468, 2000.

[119] A. Wold, R. J. Arnott, and J. B. Goodenough. Some Magnetic and Crystallographic Properties of the System $\mathrm{LaMn}_{1-x} \mathrm{Ni}_{x} \mathrm{O}_{3+\lambda}$. Journal of Applied Physics, 29(3):387-389, 1958.

[120] S. Baidya and T. Saha-Dasgupta. Electronic structure and phonons in $\mathrm{La}_{2} \mathrm{CoMnO}_{6}$ : A ferromagnetic insulator driven by Coulomb-assisted spinorbit coupling. Physical Review B, 84(3), 2011.

[121] C. Zener. Interaction between thed-Shells in the Transition Metals. II. Ferromagnetic Compounds of Manganese with Perovskite Structure. Physical Review, 82(3):403-405, 1951.

[122] P. W. Anderson and H. Hasegawa. Considerations on Double Exchange. Physical Review, 100(2):675-681, 1955.

[123] P. G. de Gennes. Effects of Double Exchange in Magnetic Crystals. Physical Review, 118(1):141-154, 1960.

[124] A. J. Millis, P. B. Littlewood, and B. I. Shraiman. Double Exchange Alone Does Not Explain the Resistivity of $\mathrm{La}_{1-x} \mathrm{Sr}_{x} \mathrm{MnO}_{3}$. Physical Review Letters, 74(25):5144-5147, 1995. 
[125] A. J. Millis. Cooperative Jahn-Teller effect and electron-phonon coupling in $\mathrm{La}_{1-x} \mathrm{~A}_{x} \mathrm{MnO}_{3}$. Physical Review B, 53(13):8434-8441, 1996.

[126] A. J. Millis, R. Mueller, and Boris I. Shraiman. Fermi-liquid-to-polaron crossover. II. Double exchange and the physics of colossal magnetoresistance. Physical Review B, 54(8):5405-5417, 1996.

[127] A. J. Millis. Lattice effects in magnetoresistive manganese perovskites. Nature, 392(6672):147-150, 1998.

[128] W. Archibald, J. S. Zhou, and J. B. Goodenough. First-order transition at $T_{\mathrm{C}}$ the orthomanganites. Physical Review B, 53(21):14445-14449, 1996.

[129] S. J. L. Billinge, R. G. DiFrancesco, G. H. Kwei, J. J. Neumeier, and J. D. Thompson. Direct Observation of Lattice Polaron Formation in the Local Structure of $\mathrm{La}_{1-x} \mathrm{Ca}_{x} \mathrm{MnO}_{3}$. Physical Review Letters, 77(4):715-718, 1996.

[130] P. Dai, Jiandi Zhang, H. A. Mook, S. H. Liou, P. A. Dowben, and E. W. Plummer. Experimental evidence for the dynamic Jahn-Teller effect in $\mathrm{La}_{0.65} \mathrm{Sr}_{0.35} \mathrm{MnO}_{3}$. Physical Review B, 54(6):R3694-R3697, 1996.

[131] G. J. Snyder, R. Hiskes, S. DiCarolis, M. R. Beasley, and T. H. Geballe. Intrinsic electrical transport and magnetic properties of $\mathrm{La}_{0.67} \mathrm{Ca}_{0.33} \mathrm{MnO}_{3}$ and $\mathrm{La}_{0.67} \mathrm{Sr}_{0.33} \mathrm{MnO}_{3} \mathrm{MOCVD}$ thin films and bulk material. Physical Review $B$, 53(21):14434-14444, 1996.

[132] C. H. Booth, F. Bridges, G. J. Snyder, and T. H. Geballe. Evidence of magnetization-dependent polaron distortion $\mathrm{La}_{1-x} \mathrm{~A}_{x} \mathrm{MnO}_{3}(\mathrm{~A}=\mathrm{Ca}, \mathrm{Pb})$. Physical Review B, 54(22):R15606-R15609, 1996.

[133] J.-S. Zhou, J. B. Goodenough, A. Asamitsu, and Y. Tokura. Pressure-Induced Polaronic to Itinerant Electronic Transition in $\mathrm{La}_{1-x} \mathrm{Ca}_{x} \mathrm{MnO}_{3}$ Crystals. Physical Review Letters, 79(17):3234-3237, 1997.

[134] Ch. Hartinger, F. Mayr, J. Deisenhofer, A. Loidl, and T. Kopp. Large and small polaron excitations in $\mathrm{La}_{0.67}(\mathrm{Ca}) \mathrm{Sr}_{0.33} \mathrm{MnO}_{3}$ films. Physical Review B, 69(10):2331, 2004.

[135] Ch. Hartinger, F. Mayr, A. Loidl, and T. Kopp. Polaronic excitations in colossal magnetoresistance manganite films. Physical Review B, 73(2):918, 2006.

[136] M. Quijada, J. Černe, J. R. Simpson, H. D. Drew, K. H. Ahn, A. J. Millis, R. Shreekala, R. Ramesh, M. Rajeswari, and T. Venkatesan. Optical conductivity of manganites: Crossover from Jahn-Teller small polaron to coherent transport in the ferromagnetic state. Physical Review B, 58(24):16093-16102, 1998. 
[137] K. H. Kim, J. H. Jung, and T. W. Noh. Polaron Absorption in a Perovskite Manganite $\mathrm{La}_{0.7} \mathrm{Ca}_{0.3} \mathrm{MnO}_{3}$. Physical Review Letters, 81(7):1517-1520, 1998.

[138] S. Mildner, J. Hoffmann, P. E. Blöchl, S. Techert, and C. Jooss. Temperatureand doping-dependent optical absorption in the small-polaron system $\mathrm{Pr}_{1-x} \mathrm{Ca}_{x} \mathrm{MnO}_{3}$. Physical Review B, 92(3):2502, 2015.

[139] M. Jaime, M. B. Salamon, M. Rubinstein, R. E. Treece, J. S. Horwitz, and D. B. Chrisey. High-temperature thermopower in $\mathrm{La}_{0.67} \mathrm{Ca}_{0.33} \mathrm{MnO}_{3}$ films: Evidence for polaronic transport. Physical Review B, 54(17):11914-11917, 1996.

[140] M. Jaime, H. T. Hardner, M. B. Salamon, M. Rubinstein, P. Dorsey, and D. Emin. Hall-Effect Sign Anomaly and Small-Polaron Conduction in $\left(\mathrm{La}_{1-x} \mathrm{Gd}_{x}\right)_{0.67} \mathrm{Ca}_{0.33} \mathrm{MnO}_{3}$. Physical Review Letters, 78(5):951-954, 1997.

[141] A. Banerjee, S. Pal, E. Rozenberg, and B. K. Chaudhuri. Adiabatic and nonadiabatic small-polaron hopping conduction in $\mathrm{La}_{1-x} \mathrm{~Pb}_{x} \mathrm{MnO}_{3}(0.0 \leq \mathrm{x} \leq 0.5)$ type oxides above the metal-semiconductor transition. Journal of Physics: Condensed Matter, 13(42):9489-9504, 2001.

[142] G.-M. Zhao, K. Conder, H. Keller, and K. A. Müller. Giant oxygen isotope shift in the magnetoresistive perovskite $\mathrm{La}_{1-x} \mathrm{Ca}_{x} \mathrm{MnO}_{3+y}$. Nature, 381(6584):676-678, 1996.

[143] A. Shengelaya, G.-M. Zhao, H. Keller, and K. A. Müller. EPR Evidence of Jahn-Teller Polaron Formation in $\mathrm{La}_{1-x} \mathrm{Ca}_{x} \mathrm{MnO}_{y}$. Physical Review Letters, 77(26):5296-5299, 1996.

[144] P. Dai, J. A. Fernandez-Baca, N. Wakabayashi, E. W. Plummer, Y. Tomioka, and Y. Tokura. Short-Range Polaron Correlations in the Ferromagnetic $\mathrm{La}_{1-x} \mathrm{Ca}_{x} \mathrm{MnO}_{3}$. Physical Review Letters, 85(12):2553-2556, 2000.

[145] C. P. Adams, J. W. Lynn, Y. M. Mukovskii, A. A. Arsenov, and D. A. Shulyatev. Charge Ordering and Polaron Formation in the Magnetoresistive Oxide $\mathrm{La}_{0.7} \mathrm{Ca}_{0.3} \mathrm{MnO}_{3}$. Physical Review Letters, 85(18):3954-3957, 2000.

[146] C. S. Nelson, M. v. Zimmermann, Y. J. Kim, J. P. Hill, Doon Gibbs, V. Kiryukhin, T. Y. Koo, S.-W. Cheong, D. Casa, B. Keimer, Y. Tomioka, Y. Tokura, T. Gog, and C. T. Venkataraman. Correlated polarons in dissimilar perovskite manganites. Physical Review B, 64(17):403, 2001.

[147] S. Mori, C. H. Chen, and S.-W. Cheong. Paired and Unpaired Charge Stripes in the Ferromagnetic Phase of $\mathrm{La}_{0.5} \mathrm{Sr}_{0.5} \mathrm{MnO}_{3}$. Physical Review Letters, 81(18):3972-3975, 1998. 
[148] L. Sudheendra, V. Moshnyaga, E. D. Mishina, B. Damaschke, Th. Rasing, and K. Samwer. Direct imaging of lattice-strain-induced stripe phases in an optimally doped manganite film. Physical Review B, 75(17), 2007.

[149] V. Moshnyaga, K. Gehrke, O. I. Lebedev, L. Sudheendra, A. Belenchuk, S. Raabe, O. Shapoval, J. Verbeeck, G. van Tendeloo, and K. Samwer. Electrical nonlinearity in colossal magnetoresistance manganite films: Relevance of correlated polarons. Physical Review B, 79(13):93, 2009.

[150] M. Michelmann, V. Moshnyaga, and K. Samwer. Colossal magnetoelastic effects at the phase transition of $\left(\mathrm{La}_{0.6} \mathrm{Pr}_{0.4}\right)_{0.7} \mathrm{Ca}_{0.3} \mathrm{MnO}_{3}$. Physical Review B, 85(1), 2012.

[151] J. S. Helton, Y. Zhao, D. A. Shulyatev, and J. W. Lynn. Damping and softening of transverse acoustic phonons in colossal magnetoresistive $\mathrm{La}_{0.7} \mathrm{Ca}_{0.3} \mathrm{MnO}_{3}$ and $\mathrm{La}_{0.7} \mathrm{Sr}_{0.3} \mathrm{MnO}_{3}$. Physical Review B, 99(2), 2019.

[152] M. Uehara, S. Mori, C. H. Chen, and S.-W. Cheong. Percolative phase separation underlies colossal magnetoresistance in mixed-valent manganites. Nature, 399(6736):560-563, 1999.

[153] D. Louca and T. Egami. Local lattice distortions in La1 $-x \mathrm{Sr}_{x} \mathrm{MnO}_{3}$ studied by pulsed neutron scattering. Physical Review B, 59(9):6193-6204, 1999.

[154] S. W. Huang, Y. T. Liu, J. M. Lee, J. M. Chen, J. F. Lee, R. W. Schoenlein, Y.-D. Chuang, and J.-Y. Lin. Polaronic effect in the X-ray absorption spectra of $\mathrm{La}_{1-x} \mathrm{Ca}_{x} \mathrm{MnO}_{3}$ manganites. Journal of Physics: Condensed Matter, 31(19):195601, 2019.

[155] J. M. De Teresa, M. R. Ibarra, P. A. Algarabel, C. Ritter, C. Marquina, J. Blasco, J. García, A. del Moral, and Z. Arnold. Evidence for magnetic polarons in the magnetoresistive perovskites. Nature, 386(6622):256-259, 1997.

[156] G.-M. Zhao, Y. S. Wang, D. J. Kang, W. Prellier, M. Rajeswari, H. Keller, T. Venkatesan, C. W. Chu, and R. L. Greene. Evidence for the immobile bipolaron formation in the paramagnetic state of the magnetoresistive manganites. Physical Review B, 62(18):R11949-R11952, 2000.

[157] A. Moreo, M. Mayr, A. Feiguin, S. Yunoki, and E. Dagotto. Giant Cluster Coexistence in Doped Manganites and Other Compounds. Physical Review Letters, 84(24):5568-5571, 2000.

[158] D. Khomskii. Phase separation, percolation and giant isotope effect in manganites. Physica B: Condensed Matter, 280(1-4):325-330, 2000.

[159] C. Şen, G.Alvarez, and E.Dagotto. Competing Ferromagnetic and ChargeOrdered States in Models for Manganites: The Origin of the Colossal Magnetoresistance Effect. Physical Review Letters, 98(12), 2007. 
[160] E. Dagotto. Open questions in CMR manganites, relevance of clustered states and analogies with other compounds including the cuprates. New Journal of Physics, 7:67, 2005.

[161] M. H. Burkhardt, M. A. Hossain, S. Sarkar, Y.-D. Chuang, A. G. Cruz Gonzalez, A. Doran, A. Scholl, A. T. Young, N. Tahir, Y. J. Choi, S.-W. Cheong, H. A. Dürr, and J. Stöhr. Imaging the First-Order Magnetic Transition in $\mathrm{La}_{0.35} \mathrm{Pr}_{0.275} \mathrm{Ca}_{0.375} \mathrm{MnO}_{3}$. Physical Review Letters, 108(23), 2012.

[162] S. Yunoki, J. Hu, A. L. Malvezzi, A. Moreo, N. Furukawa, and E. Dagotto. Phase Separation in Electronic Models for Manganites. Physical Review Letters, 80(4):845-848, 1998.

[163] D. P. Arovas, G. Gómez-Santos, and F. Guinea. Phase separation in doubleexchange systems. Physical Review B, 59(21):13569-13572, 1999.

[164] N. Mathur and P. Littlewood. Mesoscopic Texture in Manganites. Physics Today, 56(1):25-30, 2003.

[165] M. Mayr, A. Moreo, A. Vergés J., J. Arispe, A. Feiguin, and E. Dagotto. Resistivity of Mixed-Phase Manganites. Physical Review Letters, 86(1):135$138,2001$.

[166] T. Z. Ward, J. D. Budai, Z. Gai, J. Z. Tischler, Lifeng Yin, and J. Shen. Elastically driven anisotropic percolation in electronic phase-separated manganites. Nature Physics, 5(12):885-888, 2009.

[167] M. Sirena, L. Steren, and J. Guimpel. Thickness dependence of the properties of $\mathrm{La}_{0.6} \mathrm{~A}_{0.4} \mathrm{MnO}_{3}$ thin films. Thin Solid Films, 373(1-2):102-106, 2000.

[168] F. Tsui, M. C. Smoak, T. K. Nath, and C. B. Eom. Strain-dependent magnetic phase diagram of epitaxial $\mathrm{La}_{0.67} \mathrm{Sr}_{0.33} \mathrm{MnO}_{3}$ thin films. Applied Physics Letters, 76(17):2421-2423, 2000.

[169] M. Jungbauer, S. Hühn, J.-O. Krisponeit, and V. Moshnyaga. Visible range colossal magnetorefractive effect in $\left(\mathrm{La}_{1-y} \mathrm{Pr}_{y}\right)_{0.67} \mathrm{Ca}_{0.33} \mathrm{MnO}_{3}$ films. New Journal of Physics, 16(6):063034, 2014.

[170] H. L. Ju, H.-C. Sohn, and Kannan M. Krishnan. Evidence for $\mathrm{O}_{2 p}$ Hole-Driven Conductivity in $\mathrm{La}_{1-x} \mathrm{Sr}_{x} \mathrm{MnO}_{3}(0 \leq \mathrm{x} \leq 0.7)$ and $\mathrm{La}_{1-x} \mathrm{Ca}_{x} \mathrm{MnO}_{z}$ Thin Films. Physical Review Letters, 79(17):3230-3233, 1997.

[171] A. S. Alexandrov and A. M. Bratkovsky. Carrier Density Collapse and Colossal Magnetoresistance in Doped Manganites. Physical Review Letters, 82(1):141144, 1999. 
[172] A. S. Alexandrov and A. M. Bratkovsky. Theory of colossal magnetoresistance in doped manganites. Journal of Physics: Condensed Matter, 11(8):1989-2005, 1999.

[173] A. S. Alexandrov, A. M. Bratkovsky, and V. V. Kabanov. Phase Coexistence and Resistivity near the Ferromagnetic Transition of Manganites. Physical Review Letters, 96(11), 2006.

[174] A. S. Alexandrov and A. M. Bratkovsky. Colossal magneto-optical conductivity in doped manganites. Physical Review B, 60(9):6215-6218, 1999.

[175] A. S. Alexandrov and A. M. Bratkovsky. The essential interactions in oxides and spectral weight transfer in doped manganites. Journal of Physics: Condensed Matter, 11(48):L531-L539, 1999.

[176] J. E. Gordon, C. Marcenat, J. P. Franck, I. Isaac, Guanwen Zhang, R. Lortz, C. Meingast, F. Bouquet, R. A. Fisher, and N. E. Phillips. Specific heat and thermal expansion of $\mathrm{La}_{0.65} \mathrm{Ca}_{0.35} \mathrm{MnO}_{3}$ : Magnetic-field dependence, isotope effect, and evidence for a first-order phase transition. Physical Review B, 65(2), 2001.

[177] P. Graziosi, A. Gambardella, M. Prezioso, A. Riminucci, I. Bergenti, N. Homonnay, G. Schmidt, D. Pullini, and D. Busquets-Mataix. Polaron framework to account for transport properties in metallic epitaxial manganite films. Physical Review B, 89(21):1843, 2014.

[178] M. Maschek, D. Lamago, J.-P. Castellan, A. Bosak, D. Reznik, and F. Weber. Polaronic metal phases in $\mathrm{La}_{0.7} \mathrm{Sr}_{0.3} \mathrm{MnO}_{3}$ uncovered by inelastic neutron and x-ray scattering. Physical Review B, 93(4), 2016.

[179] L. Hu, L. Q. Yu, P. Xiong, X. L. Wang, J. H. Zhao, L. F. Wang, Z. Huang, and W. B. Wu. Static and dynamic signatures of anisotropic electronic phase separation in $\mathrm{La}_{0.67} \mathrm{Ca}_{0.33} \mathrm{MnO}_{3}$ thin films under anisotropic strain. Physical Review B, 97(21):894, 2018.

[180] E. Dagotto, T. Hotta, and A. Moreo. Colossal magnetoresistant materials: the key role of phase separation. Physics Reports, 344(1-3):1-153, 2001.

[181] Y. Choi, M. C. Lin, and M. Liu. Computational study on the catalytic mechanism of oxygen reduction on $\mathrm{La}_{0.5} \mathrm{Sr}_{0.5} \mathrm{MnO}_{3}$ in solid oxide fuel cells. Angewandte Chemie (International ed. in English), 46(38):7214-7219, 2007.

[182] N. Rezlescu, E. Rezlescu, C. Doroftei, P. D. Popa, and M. Ignat. Nanostructured Lanthanum Manganite Perovskites In Catalyst Applications. Digest Journal of Nanomaterials and Biostructures, 8(2):581, 2013. 
[183] W. Lee, J. W. Han, Y. Chen, Z. Cai, and B. Yildiz. Cation size mismatch and charge interactions drive dopant segregation at the surfaces of manganite perovskites. Journal of the American Chemical Society, 135(21):7909-7925, 2013.

[184] A. Ohtomo and H. Y. Hwang. A high-mobility electron gas at the $\mathrm{LaAlO}_{3} / \mathrm{SrTiO}_{3}$ heterointerface. Nature, 427(6973):423-426, 2004.

[185] A. J. Grutter, A. Vailionis, J. A. Borchers, B. J. Kirby, C. L. Flint, C. He, E. Arenholz, and Y. Suzuki. Interfacial Symmetry Control of Emergent Ferromagnetism at the Nanoscale. Nano Letters, 16(9):5647-5651, 2016.

[186] K.-Y. Meng, A. S. Ahmed, M. Baćani, A.-O. Mandru, X. Zhao, N. Bagués, B. D. Esser, J. Flores, D. W. McComb, H. J. Hug, and F. Yang. Observation of Nanoscale Skyrmions in $\mathrm{SrIrO}_{3} / \mathrm{SrRuO}_{3}$ Bilayers. Nano Letters, 19(5):3169$3175,2019$.

[187] J.-O. Krisponeit, C. Kalkert, B. Damaschke, V. Moshnyaga, and K. Samwer. Nanoscale resistance switching in manganite thin films: Sharp voltage threshold and pulse-width dependence. Physical Review B, 82(14), 2010.

[188] Ll. Abad, B. Martínez, and Ll. Balcells. Surface behavior of $\mathrm{La}_{0.67} \mathrm{Ca}_{0.33} \mathrm{MnO}_{3}$ epitaxial thin films. Applied Physics Letters, 87(21):212502, 2005.

[189] M. Huijben, L. W. Martin, Y.-H. Chu, M. B. Holcomb, P. Yu, G. Rijnders, D. H. A. Blank, and R. Ramesh. Critical thickness and orbital ordering in ultrathin $\mathrm{La}_{0.7} \mathrm{Sr}_{0.3} \mathrm{MnO}_{3}$ films. Physical Review B, 78(9), 2008.

[190] A. Biswas, M. Rajeswari, R. C. Srivastava, T. Venkatesan, R. L. Greene, Q. Lu, A. L. de Lozanne, and A. J. Millis. Strain-driven charge-ordered state in $\mathrm{La}_{0.67} \mathrm{Sr}_{0.33} \mathrm{MnO}_{3}$. Physical Review B, 63(18):3239, 2001.

[191] M. Bibes, L. Balcells, S. Valencia, J. Fontcuberta, M. Wojcik, E. Jedryka, and S. Nadolski. Nanoscale multiphase separation at $\mathrm{La}_{0.67} \mathrm{Sr}_{0.33} \mathrm{MnO}_{3} / \mathrm{SrTiO}_{3}$ interfaces. Physical Review Letters, 87(6):067210, 2001.

[192] T. Becker, C. Streng, Y. Luo, V. Moshnyaga, B. Damaschke, N. Shannon, and K. Samwer. Intrinsic inhomogeneities in manganite thin films investigated with scanning tunneling spectroscopy. Physical Review Letters, 89(23):237203, 2002 .

[193] A. A. Sidorenko, G. Allodi, R. De Renzi, G. Balestrino, and M. Angeloni. $\mathrm{Mn}^{55} \mathrm{NMR}$ and magnetization studies of $\mathrm{La}_{0.67} \mathrm{Sr}_{0.33} \mathrm{MnO}_{3}$ thin films. Physical Review B, 73(5), 2006.

[194] M. Angeloni, G. Balestrino, N. G. Boggio, P. G. Medaglia, P. Orgiani, and A. Tebano. Suppression of the metal-insulator transition temperature in thin $\mathrm{La}_{0.7} \mathrm{Sr}_{0.3} \mathrm{MnO}_{3}$ films. Journal of Applied Physics, 96(11):6387-6392, 2004. 
[195] H. Y. Hwang, Y. Iwasa, M. Kawasaki, B. Keimer, N. Nagaosa, and Y. Tokura. Emergent phenomena at oxide interfaces. Nature Materials, 11(2):103-113, 2012 .

[196] F. Bottin, F. Finocchi, and C. Noguera. Facetting and $(\mathrm{n} \times 1)$ reconstructions of $\mathrm{SrTiO}_{3}(110)$ surfaces. Surface Science, 574(1):65-76, 2005.

[197] A. Wander, I. J. Bush, and N. M. Harrison. Stability of rocksalt polar surfaces: An ab initio study of $\mathrm{MgO}(111)$ and $\mathrm{NiO}(111)$. Physical Review B, 68(23):4977, 2003.

[198] R. A. Evarestov, E. A. Kotomin, E. Heifets, J. Maier, and G. Borstel. Ab initio Hartree-Fock calculations of $\mathrm{LaMnO}_{3}$ (110) surfaces. Solid State Communications, 127(5):367-371, 2003.

[199] E. A. Kotomin, E. Heifets, J. Maier, and W. A. Goddard III. Atomistic simulations of the $\mathrm{LaMnO}_{3}$ (110) polar surface. Physical Chemistry Chemical Physics, 5(19):4180, 2003.

[200] R. A. Evarestov, E. A. Kotomin, Yu. A. Mastrikov, D. Gryaznov, E. Heifets, and J. Maier. Comparative density-functional LCAO and plane-wave calculations of $\mathrm{LaMnO}_{3}$ surfaces. Physical Review B, 72(21):231, 2005.

[201] F. L. Tang, M. Huang, W. J. Lu, and W. Y. Yu. Structural relaxation and Jahn-Teller distortion of $\mathrm{LaMnO}_{3}$ (001) surface. Surface Science, 603(6):949954, 2009.

[202] Yu. A. Mastrikov, E. Heifets, E. A. Kotomin, and J. Maier. Atomic, electronic and thermodynamic properties of cubic and orthorhombic $\mathrm{LaMnO}_{3}$ surfaces. Surface Science, 603(2):326-335, 2009.

[203] A. Filippetti and W. E. Pickett. Magnetic Reconstruction at the (001) $\mathrm{CaMnO}_{3}$ Surface. Physical Review Letters, 83(20):4184-4187, 1999.

[204] F. Hou, T.-Y. Cai, S. Ju, and M.-R. Shen. Magnetic reconstruction at oxygendeficient $\mathrm{SrMnO}_{3}$ (001) surface: A first-principle investigation. Applied Physics Letters, 99(19):192510, 2011.

[205] S. Keshavarz, Y. O. Kvashnin, D. C. M. Rodrigues, M. Pereiro, I. Di Marco, C. Autieri, L. Nordström, I. V. Solovyev, B. Sanyal, and O. Eriksson. Exchange interactions of $\mathrm{CaMnO}_{3}$ in the bulk and at the surface. Physical Review B, 95(11), 2017.

[206] J. M. Pruneda, V. Ferrari, R. Rurali, P. B. Littlewood, N. A. Spaldin, and E. Artacho. Ferrodistortive instability at the (001) surface of half-metallic manganites. Physical Review Letters, 99(22):226101, 2007. 
[207] J. Choi, J. Zhang, S.-H. Liou, P. A. Dowben, and E. W. Plummer. Surfaces of the perovskite manganites $\mathrm{La}_{1-x} \mathrm{Ca}_{x} \mathrm{MnO}_{3}$. Physical Review B, 59(20):1345313459, 1999.

[208] J. Choi, H. Dulli, S.-H. Liou, P. A. Dowben, and M. A. Langell. The Influence of Surface Terminal Layer and Surface Defects on the Electronic Structure of CMR Perovskites: $\mathrm{La}_{0.65} \mathrm{~A}_{0.35} \mathrm{MnO}_{3}(\mathrm{~A}=\mathrm{Ca}$, Sr, Ba). physica status solidi (b), 214(1):45-57, 1999 .

[209] M. Yoshimoto, H. Maruta, T. Ohnishi, K. Sasaki, and H. Koinuma. In situ determination of the terminating layer of $\mathrm{La}_{0.7} \mathrm{Sr}_{0.3} \mathrm{MnO}_{3}$ thin films using coaxial impact-collision ion scattering spectroscopy. Applied Physics Letters, 73(2):187-189, 1998.

[210] H. Kumigashira, K. Horiba, H. Ohguchi, K. Ono, M. Oshima, N. Nakagawa, M. Lippmaa, M. Kawasaki, and H. Koinuma. In situ photoemission characterization of terminating-layer-controlled $\mathrm{La}_{0.6} \mathrm{Sr}_{0.4} \mathrm{MnO}_{3}$ thin films. Applied Physics Letters, 82(20):3430-3432, 2003.

[211] D. Pesquera, G. Herranz, A. Barla, E. Pellegrin, F. Bondino, E. Magnano, F. Sánchez, and J. Fontcuberta. Surface symmetry-breaking and strain effects on orbital occupancy in transition metal perovskite epitaxial films. Nature Communications, 3:1189, 2012.

[212] D. Cappus, M. Haßel, E. Neuhaus, M. Heber, F. Rohr, and H.-J. Freund. Polar surfaces of oxides: reactivity and reconstruction. Surface Science, 337(3):268$277,1995$.

[213] R. Bertacco, J. P. Contour, A. Barthélemy, and J. Olivier. Evidence for strontium segregation in $\mathrm{La}_{0.7} \mathrm{Sr}_{0.3} \mathrm{MnO}_{3}$ thin films grown by pulsed laser deposition: consequences for tunnelling junctions. Surface Science, 511(1-3):366-372, 2002 .

[214] Z. Li, M. Bosman, Z. Yang, P. Ren, L. Wang, L. Cao, X. Yu, C. Ke, M. B. H. Breese, A. Rusydi, W. Zhu, Z. Dong, and Y. L. Foo. Interface and Surface Cation Stoichiometry Modified by Oxygen Vacancies in Epitaxial Manganite Films. Advanced Functional Materials, 22(20):4312-4321, 2012.

[215] T. T. Fister, D. D. Fong, J. A. Eastman, P. M. Baldo, M. J. Highland, P. H. Fuoss, K. R. Balasubramaniam, J. C. Meador, and P. A. Salvador. In situ characterization of strontium surface segregation in epitaxial $\mathrm{La}_{0.7} \mathrm{Sr}_{0.3} \mathrm{MnO}_{3}$ thin films as a function of oxygen partial pressure. Applied Physics Letters, 93(15):151904, 2008.

[216] A. Tselev, R. K. Vasudevan, A. G. Gianfrancesco, L. Qiao, P. Ganesh, T. L. Meyer, H. N. Lee, M. D. Biegalski, A. P. Baddorf, and S. V. Kalinin. Surface Control of Epitaxial Manganite Films via Oxygen Pressure. ACS Nano, 9(4):4316-4327, 2015. 
[217] W. Yuan, Y. Zhao, C. Tang, T. Su, Q. Song, J. Shi, and W. Han. Epitaxial growth and properties of $\mathrm{La}_{0.7} \mathrm{Sr}_{0.3} \mathrm{MnO}_{3}$ thin films with micrometer wide atomic terraces. Applied Physics Letters, 107(2):022404, 2015.

[218] K. Fuchigami, Z. Gai, T. Z. Ward, L. F. Yin, P. C. Snijders, E. W. Plummer, and J. Shen. Tunable metallicity of the $\mathrm{La}_{0.75} \mathrm{Sr}_{0.25} \mathrm{MnO}_{3}$ (001) surface by an oxygen overlayer. Physical Review Letters, 102(6):066104, 2009.

[219] S. Estradé, J. Arbiol, F. Peiró, Ll. Abad, V. Laukhin, Ll. Balcells, and B. Martínez. Cationic diffusion in $\mathrm{La}_{0.67} \mathrm{Ca}_{0.33} \mathrm{MnO}_{3}$ thin films grown on $\mathrm{LaAlO}_{3}$ (001) substrates. Applied Physics Letters, 91(25):252503, 2007.

[220] S. Estradé, J. Arbiol, F. Peiró, I. C. Infante, F. Sánchez, J. Fontcuberta, F. de La Peña, M. Walls, and C. Colliex. Cationic and charge segregation in $\mathrm{La}_{0.67} \mathrm{Sr}_{0.33} \mathrm{MnO}_{3}$ thin films grown on (001) and (110) $\mathrm{SrTiO}_{3}$. Applied Physics Letters, 93(11):112505, 2008.

[221] S. Estradé, J. M. Rebled, J. Arbiol, F. Peiró, I. C. Infante, G. Herranz, F. Sánchez, J. Fontcuberta, R. Córdoba, B. G. Mendis, and A. L. Bleloch. Effects of thickness on the cation segregation in epitaxial (001) and (110) $\mathrm{La}_{0.67} \mathrm{Ca}_{0.33} \mathrm{MnO}_{3}$ thin films. Applied Physics Letters, 95(7):072507, 2009.

[222] C. Kwon, M. C. Robson, K.-C. Kim, J. Y. Gu, S. E. Lofland, S. M. Bhagat, Z. Trajanovic, M. Rajeswari, T. Venkatesan, A. R. Kratz, R. D. Gomez, and R. Ramesh. Stress-induced effects in epitaxial $\mathrm{La}_{0.7} \mathrm{Sr}_{0.3} \mathrm{MnO}_{3}$ films. Journal of Magnetism and Magnetic Materials, 172(3):229-236, 1997.

[223] Y. Suzuki, H. Y. Hwang, S.-W. Cheong, and R. B. van Dover. The role of strain in magnetic anisotropy of manganite thin films. Applied Physics Letters, 71(1):140-142, 1997.

[224] P. Adamo, X. Ke, H. Q. Wang, H. L. Xin, T. Heeg, M. E. Hawley, W. Zander, J. Schubert, P. Schiffer, D. A. Muller, L. Maritato, and D. G. Schlom. Effect of biaxial strain on the electrical and magnetic properties of (001) $\mathrm{La}_{0.7} \mathrm{Sr}_{0.3} \mathrm{MnO}_{3}$ thin films. Applied Physics Letters, 95(11):112504, 2009.

[225] Z. Liao, F. Li, P. Gao, L. Li, J. Guo, X. Pan, R. Jin, E. W. Plummer, and J. Zhang. Origin of the metal-insulator transition in ultrathin films of $\mathrm{La}_{0.67} \mathrm{Sr}_{0.33} \mathrm{MnO}_{3}$. Physical Review B, 92(12), 2015.

[226] C. Aruta, G. Ghiringhelli, A. Tebano, N. G. Boggio, N. B. Brookes, P. G. Medaglia, and G. Balestrino. Strain induced x-ray absorption linear dichroism in $\mathrm{La}_{0.7} \mathrm{Sr}_{0.3} \mathrm{MnO}_{3}$ thin films. Physical Review B, 73(23):220, 2006.

[227] A. Tebano, C. Aruta, S. Sanna, P. G. Medaglia, G. Balestrino, A. A. Sidorenko, R. de Renzi, G. Ghiringhelli, L. Braicovich, V. Bisogni, and N. B. Brookes. Evidence of orbital reconstruction at interfaces in ultrathin $\mathrm{La}_{0.67} \mathrm{Sr}_{0.33} \mathrm{MnO}_{3}$ films. Physical Review Letters, 100(13):137401, 2008. 
[228] C. Aruta, G. Ghiringhelli, V. Bisogni, L. Braicovich, N. B. Brookes, A. Tebano, and G. Balestrino. Orbital occupation, atomic moments, and magnetic ordering at interfaces of manganite thin films. Physical Review B, 80(1):136, 2009.

[229] A. Tebano, A. Orsini, P. G. Medaglia, D. Di Castro, G. Balestrino, B. Freelon, A. Bostwick, Young Jun Chang, G. Gaines, E. Rotenberg, and N. L. Saini. Preferential occupation of interface bands in $\mathrm{La}_{0.67} \mathrm{Sr}_{0.33} \mathrm{MnO}_{3}$ films as seen via angle-resolved photoemission. Physical Review B, 82(21), 2010.

[230] B. Cui, C. Song, F. Li, G. Y. Wang, H. J. Mao, J. J. Peng, F. Zeng, and F. Pan. Tuning the entanglement between orbital reconstruction and charge transfer at a film surface. Scientific Reports, 4:4206, 2014.

[231] J. M. Rondinelli and N. A. Spaldin. Structure and properties of functional oxide thin films: insights from electronic-structure calculations. Advanced Materials, 23(30):3363-3381, 2011.

[232] J. M. Rondinelli, S. J. May, and J. W. Freeland. Control of octahedral connectivity in perovskite oxide heterostructures: An emerging route to multifunctional materials discovery. MRS Bulletin, 37(3):261-270, 2012.

[233] X. Li, I. Lindfors-Vrejoiu, M. Ziese, A. Gloter, and P. A. van Aken. Impact of interfacial coupling of oxygen octahedra on ferromagnetic order in $\mathrm{La}_{0.7} \mathrm{Sr}_{0.3} \mathrm{MnO}_{3} / \mathrm{SrTiO}_{3}$ heterostructures. Scientific Reports, 7:40068, 2017.

[234] E. J. Moon, P. V. Balachandran, B. J. Kirby, D. J. Keavney, R. J. SichelTissot, C. M. Schlepütz, E. Karapetrova, X. M. Cheng, J. M. Rondinelli, and S. J. May. Effect of interfacial octahedral behavior in ultrathin manganite films. Nano Letters, 14(5):2509-2514, 2014.

[235] S. Koohfar, A. S. Disa, M. S. J. Marshall, F. J. Walker, C. H. Ahn, and D. P. Kumah. Structural distortions at polar manganite interfaces. Physical Review $B, 96(2), 2017$.

[236] L. Abad, V. Laukhin, S. Valencia, A. Gaup, W. Gudat, L. Balcells, and B. Martínez. Interfacial Strain: The Driving Force for Selective Orbital Occupancy in Manganite Thin Films. Advanced Functional Materials, 17(18):3918$3925,2007$.

[237] R. Herger, P. R. Willmott, C. M. Schlepütz, M. Björck, S. A. Pauli, D. Martoccia, B. D. Patterson, D. Kumah, R. Clarke, Y. Yacoby, and M. Döbeli. Structure determination of monolayer-by-monolayer grown $\mathrm{La}_{1-x} \mathrm{Sr}_{x} \mathrm{MnO}_{3}$ thin films and the onset of magnetoresistance. Physical Review B, 77(8), 2008.

[238] T. Riedl, T. Gemming, K. Dörr, M. Luysberg, and K. Wetzig. Mn Valency at $\mathrm{La}_{0.7} \mathrm{Sr}_{0.3} \mathrm{MnO}_{3} / \mathrm{SrTiO}_{3}$ (001) Thin Film Interfaces. Microscopy and Microanalysis, 15(3):213-221, 2009. 
[239] F. Y. Bruno, J. Garcia-Barriocanal, M. Varela, N. M. Nemes, P. Thakur, J. C. Cezar, N. B. Brookes, A. Rivera-Calzada, M. Garcia-Hernandez, C. Leon, S. Okamoto, S. J. Pennycook, and J. Santamaria. Electronic and Magnetic Reconstructions $\mathrm{La}_{0.7} \mathrm{Sr}_{0.3} \mathrm{MnO}_{3} / \mathrm{SrTiO}_{3}$ Heterostructures: A Case of Enhanced Interlayer Coupling Controlled by the Interface. Physical Review Letters, 106(14), 2011.

[240] J.-O. Krisponeit, B. Damaschke, V. Moshnyaga, and K. Samwer. Layerby-Layer Resistive Switching: Multistate Functionality due to Electric-FieldInduced Healing of Dead Layers. Physical Review Letters, 122(13), 2019.

[241] Y. Anahory, L. Embon, C. J. Li, S. Banerjee, A. Meltzer, H. R. Naren, A. Yakovenko, J. Cuppens, Y. Myasoedov, M. L. Rappaport, M. E. Huber, K. Michaeli, T. Venkatesan, Ariando, and E. Zeldov. Emergent nanoscale superparamagnetism at oxide interfaces. Nature Communications, 7(1), 2016.

[242] S. Valencia, M. J. Calderón, L. López-Mir, Z. Konstantinovic, E. Schierle, E. Weschke, L. Brey, B. Martínez, and Ll. Balcells. Enhancement of spinorbit coupling at manganite surfaces. Physical Review B, 98(11), 2018.

[243] A. Soumyanarayanan, N. Reyren, A. Fert, and C. Panagopoulos. Emergent phenomena induced by spin-orbit coupling at surfaces and interfaces. Nature, 539(7630):509-517, 2016.

[244] H. Kuzmany. Solid-State Spectroscopy. Springer Berlin Heidelberg, 2014.

[245] D. A. Long. Raman Spectroscopy. McGraw-Hill, 1977.

[246] E. Le Ru and P. Etchegoin. Principles of Surface-Enhanced Raman Spectroscopy: And Related Plasmonic Effects. Elsevier Science, 2008.

[247] F. Cerdeira, T. A. Fjeldly, and M. Cardona. Interaction between electronic and vibronic Raman scattering in heavily doped silicon. Solid State Communications, 13(3):325-328, 1973.

[248] M. Cardona. Light Scattering in Solids I. Springer Berlin Heidelberg, 1983.

[249] S. Yoon, H. L. Liu, G. Schollerer, S. L. Cooper, P. D. Han, D. A. Payne, S.-W. Cheong, and Z. Fisk. Raman and optical spectroscopic studies of smallto-large polaron crossover in the perovskite manganese oxides. Physical Review $B, 58(5): 2795-2801,1998$.

[250] X. K. Chen, J. G. Naeini, K. C. Hewitt, J. C. Irwin, R. Liang, and W. N. Hardy. Electronic Raman scattering in underdoped $\mathrm{YBa}_{2} \mathrm{Cu}_{3} \mathrm{O}_{6.5}$. Physical Review B, 56(2):R513-R516, 1997. 
[251] M. Opel, R. Nemetschek, C. Hoffmann, R. Philipp, P. F. Müller, R. Hackl, I. Tüttő, A. Erb, B. Revaz, E. Walker, H. Berger, and L. Forró. Carrier relaxation, pseudogap, and superconducting gap in high- $T_{\mathrm{C}}$ cuprates: A Raman scattering study. Physical Review B, 61(14):9752-9774, 2000.

[252] G. R. Boyd, T. P. Devereaux, P. J. Hirschfeld, V. Mishra, and D. J. Scalapino. Probing the pairing symmetry of the iron pnictides with electronic Raman scattering. Physical Review B, 79(17), 2009.

[253] N. S. Mueller, S. Heeg, and S. Reich. Surface-enhanced Raman scattering as a higher-order Raman process. Physical Review A, 94(2), 2016.

[254] M. K. Schmidt, R. Esteban, A. González-Tudela, G. Giedke, and J. Aizpurua. Quantum Mechanical Description of Raman Scattering from Molecules in Plasmonic Cavities. ACS Nano, 10(6):6291-6298, 2016.

[255] J. M. Hollas. Modern Spectroscopy. John Wiley \& Sons Inc, 2003.

[256] C. P. Brock, Th. Hahn, H. Wondratschek, U. Müller, U. Shmueli, E. Prince, A. Authier, V. Kopský, D. B. Litvin, E. Arnold, D. M. Himmel, M. G. Rossmann, S. Hall, B. McMahon, M. I. Aroyo, C. J. Gilmore, J. A. Kaduk, and H. Schenk, editors. International Tables for Crystallography. International Union of Crystallography, 2016.

[257] B. A. DeAngelis and William B. Newnham, R.E. Factor Group Analysis of the Vibrational Spectra of Crystals: A Review and Consolidation. American Mineralogist, 578:255, 1972.

[258] M. V. Abrashev, A. P. Litvinchuk, M. N. Iliev, R. L. Meng, V. N. Popov, V. G. Ivanov, R. A. Chakalov, and C. Thomsen. Comparative study of optical phonons in the rhombohedrally distorted perovskite $\mathrm{LaMnO}_{3}$ and $\mathrm{LaAlO}_{3}$. Physical Review B, 59(6):4146-4153, 1999.

[259] V. A. Amelitchev, B. Güttler, O. Yu. Gorbenko, A. R. Kaul, A. A. Bosak, and A. Yu. Ganin. Structural and chemical analysis of colossal magnetoresistance manganites by Raman spectrometry. Physical Review B, 63(10):589, 2001.

[260] S. Dattagupta and A. Sood. Effect of charge ordering/disordering on Raman line shape in manganites. Physical Review B, 65(6):181, 2002.

[261] S. Mansouri, S. Charpentier, S. Jandl, P. Fournier, A. A. Mukhin, V. Yu Ivanov, and A. Balbashov. A micro-Raman study of a $\operatorname{Pr}_{0.5} \mathrm{Ca}_{0.5} \mathrm{MnO}_{3}$ single crystal and thin films. Journal of Physics: Condensed Matter, 21(38):386004, 2009 .

[262] L. Martín-Carrón, A. de Andrés, M. J. Martínez-Lope, M. T. Casais, and J. A. Alonso. Raman phonons as a probe of disorder, fluctuations, and local 
structure in doped and undoped orthorhombic and rhombohedral manganites. Physical Review B, 66(17):413, 2002.

[263] J. C. Irwin, J. Chrzanowski, and J. P. Franck. Oxygen isotope effect on the vibrational modes of $\mathrm{La}_{1-x} \mathrm{Ca}_{x} \mathrm{MnO}_{3}$. Physical Review B, 59(14):9362-9371, 1999.

[264] A. Antonakos, E. Liarokapis, G. H. Aydogdu, and H.-U. Habermeier. Strain effects on $\mathrm{La}_{0.5} \mathrm{Ca}_{0.5} \mathrm{MnO}_{3}$ thin films. Materials Science and Engineering: $B$, 144(1-3):83-88, 2007.

[265] A. Antonakos, D. Palles, E. Liarokapis, M. Filippi, and W. Prellier. Evaluation of the strains in charge-ordered $\mathrm{Pr}_{1-x} \mathrm{Ca}_{x} \mathrm{MnO}_{3}$ thin films using Raman spectroscopy. Journal of Applied Physics, 104(6):063508, 2008.

[266] A. Antonakos, E. Liarokapis, M. Filippi, and W. Prellier. Optical Study of the Strain Effects on $\mathrm{La}_{0.6} \mathrm{Ca}_{0.4} \mathrm{MnO}_{3}$ Thin Films. Journal of Superconductivity and Novel Magnetism, 22(3):209-213, 2009.

[267] E. Liarokapis, Th. Leventouri, D. Lampakis, D. Palles, J. J. Neumeier, and D. H. Goodwin. Local lattice distortions and Raman spectra in the La1 - $x \mathrm{Ca}_{x} \mathrm{MnO}_{3}$ system. Physical Review B, 60(18):12758-12763, 1999.

[268] S. Merten, O. Shapoval, B. Damaschke, K. Samwer, and V. Moshnyaga. Magnetic-Field-Induced Suppression of Jahn-Teller Phonon Bands in $\left(\mathrm{La}_{0.6} \mathrm{Pr}_{0.4}\right)_{0.7} \mathrm{Ca}_{0.3} \mathrm{MnO}_{3}$ : the Mechanism of Colossal Magnetoresistance shown by Raman Spectroscopy. Scientific Reports, 9(1), 2019.

[269] LabRAM HR User Manual.

[270] Microstat MO (Oxford Instruments) - User Manual.

[271] M. Fleischmann, P. J. Hendra, and A. J. McQuillan. Raman spectra of pyridine adsorbed at a silver electrode. Chemical Physics Letters, 26(2):163-166, 1974.

[272] D. L. Jeanmaire and R. P. Van Duyne. Surface Raman Spectroelectrochemistry. Journal of Electroanalytical Chemistry and Interfacial Electrochemistry, 84(1):1-20, 1977.

[273] M. G. Albrecht and J. A. Creighton. Anomalously intense Raman spectra of pyridine at a silver electrode. Journal of the American Chemical Society, 99(15):5215-5217, 1977.

[274] S. Enoch and N. Bonod. Plasmonics: From Basics to Advanced Topics (Springer Series in Optical Sciences). Springer, 2015.

[275] V. Amendola, R. Pilot, M. Frasconi, O. M. Maragò, and M. A. Iatì. Surface plasmon resonance in gold nanoparticles: a review. Journal of Physics: Condensed Matter, 29(20):203002, 2017. 
[276] K. L. Kelly, E. Coronado, L. L. Zhao, and G. C. Schatz. The Optical Properties of Metal Nanoparticles: The Influence of Size, Shape, and Dielectric Environment. The Journal of Physical Chemistry B, 107(3):668-677, 2003.

[277] A. Wokaun, J. P. Gordon, and P. F. Liao. Radiation Damping in SurfaceEnhanced Raman Scattering. Physical Review Letters, 48(14):957-960, 1982.

[278] B. N. J. Persson, D. Schumacher, and A. Otto. Surface resistivity and vibrational damping in adsorbed layers. Chemical Physics Letters, 178(2-3):204$212,1991$.

[279] P. K. Jain, K. S. Lee, I. H. El-Sayed, and M. A. El-Sayed. Calculated Absorption and Scattering Properties of Gold Nanoparticles of Different Size, Shape, and Composition: Applications in Biological Imaging and Biomedicine. The Journal of Physical Chemistry B, 110(14):7238-7248, 2006.

[280] S. E. J. Bell and M. R. McCourt. SERS enhancement by aggregated Au colloids: effect of particle size. Physical Chemistry Chemical Physics, 11(34):7455, 2009 .

[281] A. Derkachova, K. Kolwas, and I. Demchenko. Dielectric Function for Gold in Plasmonics Applications: Size Dependence of Plasmon Resonance Frequencies and Damping Rates for Nanospheres. Plasmonics, 11(3):941-951, 2015.

[282] B. N. J. Persson. Polarizability of small spherical metal particles: influence of the matrix environment. Surface Science, 281(1-2):153-162, 1993.

[283] M. M. Miller and A. A. Lazarides. Sensitivity of Metal Nanoparticle Surface Plasmon Resonance to the Dielectric Environment. The Journal of Physical Chemistry B, 109(46):21556-21565, 2005.

[284] J. Gersten and A. Nitzan. Electromagnetic theory of enhanced Raman scattering by molecules adsorbed on rough surfaces. The Journal of Chemical Physics, 73(7):3023-3037, 1980.

[285] A. Otto, I. Mrozek, H. Grabhorn, and W. Akemann. Surface-enhanced Raman scattering. Journal of Physics: Condensed Matter, 4(5):1143-1212, 1992.

[286] K. A. Willets and R. P. Van Duyne. Localized Surface Plasmon Resonance Spectroscopy and Sensing. Annual Review of Physical Chemistry, 58(1):267297, 2007.

[287] S. Schlücker. Oberflächenverstärkte Raman-Spektroskopie: Konzepte und chemische Anwendungen. Angewandte Chemie, 126(19):4852-4894, 2014.

[288] J. D. Jackson. Classical Electrodynamics. John Wiley \& Sons Inc, 1998. 
[289] C. F. Bohren and D. R. Huffman. Absorption and Scattering of Light by Small Particles. Wiley, 1998.

[290] M. I. Mishchenko, L. D. Travis, and A. A. Lacis. Scattering, Absorption, and Emission of Light by Small Particles. Cambridge University Press, 2002.

[291] T. M. Cotton, R. A. Uphaus, and D. Mobius. Distance dependence of surfaceenhanced resonance Raman enhancement in Langmuir-Blodgett dye multilayers. The Journal of Physical Chemistry, 90(23):6071-6073, 1986.

[292] J. A. Dieringer, A. D. McFarland, N. C. Shah, D. A. Stuart, A. V. Whitney, C. R. Yonzon, M. A. Young, X. Zhang, and R. P. Van Duyne. Introductory Lecture : Surface enhanced Raman spectroscopy: new materials, concepts, characterization tools, and applications. Faraday Discussions, 132:9-26, 2006.

[293] E. Hao and G. C. Schatz. Electromagnetic fields around silver nanoparticles and dimers. The Journal of Chemical Physics, 120(1):357-366, 2004.

[294] I. W. Sztainbuch. The effects of Au aggregate morphology on surfaceenhanced Raman scattering enhancement. The Journal of Chemical Physics, 125(12):124707, 2006.

[295] M. Moskovits. Surface roughness and the enhanced intensity of Raman scattering by molecules adsorbed on metals. The Journal of Chemical Physics, 69(9):4159-4161, 1978.

[296] P. N. Sanda, J. M. Warlaumont, J. E. Demuth, J. C. Tsang, K. Christmann, and J. A. Bradley. Surface-Enchanced Raman Scattering from Pyridine on Ag(111). Physical Review Letters, 45(18):1519-1523, 1980.

[297] Yu, S.-S. Chang, L. Lee, C, and C. R. C. Wang. Gold Nanorods: Electrochemical Synthesis and Optical Properties. The Journal of Physical Chemistry B, 101(34):6661-6664, 1997.

[298] S. Chen, Z. Fan, and D. L. Carroll. Silver Nanodisks: Synthesis, Characterization, and Self-Assembly. The Journal of Physical Chemistry B, 106(42):1077710781, 2002.

[299] A. V. Whitney, B. D. Myers, and R. P. Van Duyne. Sub-100 nm Triangular Nanopores Fabricated with the Reactive Ion Etching Variant of Nanosphere Lithography and Angle-Resolved Nanosphere Lithography. Nano Letters, 4(8):1507-1511, 2004.

[300] L.-W. Liu, Q.-W. Zhou, Z.-Q. Zeng, M.-L. Jin, G.-F. Zhou, R.-Z. Zhan, H.-J. Chen, X.-S. Gao, X.-B. Lu, S. Senz, Z. Zhang, and J.-M. Liu. Induced SERS activity in $\mathrm{Ag} @ \mathrm{SiO}_{2} / \mathrm{Ag}$ core-shell nanosphere arrays with tunable interior insulator. Journal of Raman Spectroscopy, 47(10):1200-1206, 2016. 
[301] T. Jensen, L. Kelly, A. Lazarides, and G. C. Schatz. Electrodynamics of Noble Metal Nanoparticles and Nanoparticle Clusters. Journal of Cluster Science, 10(2):295-317, 1999.

[302] T. Yamaguchi, S. Yoshida, and A. Kinbara. Optical effect of the substrate on the anomalous absorption of aggregated silver films. Thin Solid Films, 21(1):173-187, 1974.

[303] U. Kreibig and M. Vollmer. Optical Properties of Metal Clusters. Springer Berlin Heidelberg, 1995.

[304] W. Hergert and T. Wriedt. The Mie Theory. Springer Berlin Heidelberg, 2012.

[305] Q. Fu and W. Sun. Mie theory for light scattering by a spherical particle in an absorbing medium. Applied Optics, 40(9):1354, 2001.

[306] L. L. Zhao, K. L. Kelly, and G. C. Schatz. The Extinction Spectra of Silver Nanoparticle Arrays: Influence of Array Structure on Plasmon Resonance Wavelength and Width. The Journal of Physical Chemistry B, 107(30):73437350, 2003.

[307] E. C. Le Ru, P. G. Etchegoin, and M. Meyer. Enhancement factor distribution around a single surface-enhanced Raman scattering hot spot and its relation to single molecule detection. The Journal of Chemical Physics, 125(20):204701, 2006.

[308] A. M. Michaels, J. Jiang, and L. Brus. Ag Nanocrystal Junctions as the Site for Surface-Enhanced Raman Scattering of Single Rhodamine 6G Molecules. The Journal of Physical Chemistry B, 104(50):11965-11971, 2000.

[309] H. Xu, J. Aizpurua, M. Käll, and P. Apell. Electromagnetic contributions to single-molecule sensitivity in surface-enhanced Raman scattering. Physical Review E, 62(3):4318-4324, 2000.

[310] M. Futamata. Single molecule sensitivity in SERS: importance of junction of adjacent Ag nanoparticles. Faraday Discussions, 132:45-61, 2006.

[311] E. C. Le Ru and P. G. Etchegoin. Sub-wavelength localization of hot-spots in SERS. Chemical Physics Letters, 396(4-6):393-397, 2004.

[312] R. Lässer and N. V. Smith. Interband optical transitions in gold in the photon energy range 2-25 eV. Solid State Communications, 37(6):507-509, 1981.

[313] L. D. Landau. On the Vibrations of the Electronic Plasma. In Collected Papers of L.D. Landau, pages 445-460. Elsevier, 1965.

[314] N. E. Christensen. The Band Structure of Silver and Optical Interband Transitions. physica status solidi (b), 54(2):551-563, 1972. 
[315] M. M. Alvarez, J. T. Khoury, T. G. Schaaff, M. N. Shafigullin, I. Vezmar, and R. L. Whetten. Optical Absorption Spectra of Nanocrystal Gold Molecules. The Journal of Physical Chemistry B, 101(19):3706-3712, 1997.

[316] D. Gall. Electron mean free path in elemental metals. Journal of Applied Physics, 119(8):085101, 2016.

[317] A. Otto. Theory of First Layer and Single Molecule Surface Enhanced Raman Scattering (SERS). physica status solidi (a), 188(4):1455-1470, 2001.

[318] J. R. Lombardi and R. L. Birke. A Unified Approach to Surface-Enhanced Raman Spectroscopy. The Journal of Physical Chemistry C, 112(14):56055617, 2008.

[319] A. Champion and P. Kambhampati. Surface-enhanced Raman scattering. Chemical Society Reviews, 27(4):241, 1998.

[320] A. J. Bard. Encyclopedia of Electrochemistry. Wiley-VCH Verlag GmbH \& Co. KGaA, 2007.

[321] F. J. Adrian. Charge transfer effects in surface-enhanced Raman scattering. The Journal of Chemical Physics, 77(11):5302-5314, 1982.

[322] J. R. Lombardi, R. L. Birke, T. Lu, and J. Xu. Charge-transfer theory of surface enhanced Raman spectroscopy: Herzberg-Teller contributions. The Journal of Chemical Physics, 84(8):4174-4180, 1986.

[323] A. Otto. The 'chemical' (electronic) contribution to surface-enhanced Raman scattering. Journal of Raman Spectroscopy, 36(6-7):497-509, 2005.

[324] S. M. Morton and L. Jensen. Understanding the Molecule-Surface Chemical Coupling in SERS. Journal of the American Chemical Society, 131(11):40904098, 2009.

[325] H. Grabhorn and A. Otto. What determines the selection rules of surfaceenhanced Raman spectroscopy? Vacuum, 41(1-3):473-475, 1990.

[326] P. Kambhampati, C. M. Child, M. C. Foster, and A. Campion. On the chemical mechanism of surface enhanced Raman scattering: Experiment and theory. The Journal of Chemical Physics, 108(12):5013-5026, 1998.

[327] V. Perebeinos and P. B. Allen. Multiphonon resonant Raman scattering predicted in $\mathrm{LaMnO}_{3}$ from the Franck-Condon process via self-trapped excitons. Physical Review B, 64(8):362, 2001.

[328] R. Krüger, B. Schulz, S. Naler, R. Rauer, D. Budelmann, J. Bäckström, K. H. Kim, S.-W. Cheong, V. Perebeinos, and M. Rübhausen. Orbital ordering in $\mathrm{LaMnO}_{3}$ investigated by resonance Raman spectroscopy. Physical Review Letters, 92(9):097203, 2004. 
[329] L. Martín-Carrón and A. de Andrés. Melting of the cooperative Jahn-Teller distortion in $\mathrm{LaMnO}_{3}$ single crystal studied by Raman spectroscopy. The European Physical Journal B, 22(1):11-16, 2001.

[330] R. Gupta, G. Venketeswara Pai, A. K. Sood, T. V. Ramakrishnan, and C. N. R. Rao. Raman scattering in charge-ordered $\operatorname{Pr}_{0.67} \mathrm{Ca}_{0.33} \mathrm{MnO}_{3}$ : Anomalous temperature dependence of linewidth. Europhysics Letters (EPL), 58(5):778-784, 2002.

[331] V. Dediu, C. Ferdeghini, F. C. Matacotta, P. Nozar, and G. Ruani. JahnTeller Dynamics in Charge-Ordered Manganites from Raman Spectroscopy. Physical Review Letters, 84(19):4489-4492, 2000.

[332] C. W. Searle and S. T. Wang. Studies of the ionic ferromagnet (LaPb)MnO3. V. Electric transport and ferromagnetic properties. Canadian Journal of Physics, 48(17):2023-2031, 1970.

[333] P. Schiffer, A. P. Ramirez, W. Bao, and S.-W. Cheong. Low Temperature Magnetoresistance and the Magnetic Phase Diagram of $\mathrm{La}_{1-x} \mathrm{Ca}_{x} \mathrm{MnO}_{3}$. Physical Review Letters, 75(18):3336-3339, 1995.

[334] V. Moshnyaga, L. Sudheendra, O. I. Lebedev, S. A. Köster, K. Gehrke, O. Shapoval, A. Belenchuk, B. Damaschke, G. van Tendeloo, and K. Samwer. A-Site Ordering versus Electronic Inhomogeneity in Colossally Magnetoresistive Manganite Films. Physical Review Letters, 97(10), 2006.

[335] M. M. Seikh, C. Narayana, L. Sudheendra, A. K. Sood, and C. N. R. Rao. A Brillouin scattering study of $\mathrm{La}_{0.77} \mathrm{~A}_{0.23} \mathrm{MnO}_{3}$ across the metal-insulator transition. Journal of Physics: Condensed Matter, 16(24):4381-4390, 2004.

[336] V. Kiryukhin, T. Y. Koo, H. Ishibashi, J. P. Hill, and S.-W. Cheong. Average lattice symmetry and nanoscale structural correlations in magnetoresistive manganites. Physical Review B, 67(6), 2003.

[337] J. Tao, D. Niebieskikwiat, M. Varela, W. Luo, M. A. Schofield, Y. Zhu, M. B. Salamon, J. M. Zuo, S. T. Pantelides, and S. J. Pennycook. Direct Imaging of Nanoscale Phase Separation in $\mathrm{La}_{0.55} \mathrm{~A}_{0.45} \mathrm{MnO}_{3}$ : Relationship to Colossal Magnetoresistance. Physical Review Letters, 103(9), 2009.

[338] J. Burgy, M. Mayr, V. Martin-Mayor, A. Moreo, and E. Dagotto. Colossal Effects in Transition Metal Oxides Caused by Intrinsic Inhomogeneities. Physical Review Letters, 87(27), 2001.

[339] N. A. Babushkina, L. M. Belova, D. I. Khomskii, K. I. Kugel, O. Yu. Gorbenko, and A. R. Kaul. Low-temperature transition to a metallic state in $\left(\mathrm{La}_{0.5} \mathrm{Pr}_{0.5}\right)_{0.7} \mathrm{Ca}_{0.3} \mathrm{MnO}_{3}$ films. Physical Review B, 59(10):6994-7000, 1999. 
[340] P. Levy, F. Parisi, M. Quintero, L. Granja, J. Curiale, J. Sacanell, G. Leyva, G. Polla, R. S. Freitas, and L. Ghivelder. Nonvolatile magnetoresistive memory in phase separated $\mathrm{La}_{0.25} \operatorname{Pr}_{0.3} \mathrm{Ca}_{0.375} \mathrm{MnO}_{3}$. Physical Review B, 65(14), 2002.

[341] C. P. Bean and J. D. Livingston. Superparamagnetism. Journal of Applied Physics, 30(4):S120-S129, 1959.

[342] J. W. Lynn, R. W. Erwin, J. A. Borchers, Q. Huang, A. Santoro, J.-L. Peng, and Z. Y. Li. Unconventional Ferromagnetic Transition in La1 $-x \mathrm{Ca}_{x} \mathrm{MnO}_{3}$. Physical Review Letters, 76(21):4046-4049, 1996.

[343] Y. Murakami, H. Kasai, J. J. Kim, S. Mamishin, D. Shindo, S. Mori, and A. Tonomura. Ferromagnetic domain nucleation and growth in colossal magnetoresistive manganite. Nature Nanotechnology, 5(1):37-41, 2009.

[344] N. D. Mathur, M.-H. Jo, J. E. Evetts, and M. G. Blamire. Magnetic anisotropy of thin film $\mathrm{La}_{0.7} \mathrm{Ca}_{0.3} \mathrm{MnO}_{3}$ on untwinned paramagnetic $\mathrm{NdGaO}_{3}$ (001). Journal of Applied Physics, 89(6):3388-3392, 2001.

[345] S. S. P. Parkin, R. Bhadra, and K. P. Roche. Oscillatory magnetic exchange coupling through thin copper layers. Physical Review Letters, 66(16):21522155, 1991.

[346] W. Pan, N.-Y. Jih, C.-C. Kuo, and M.-T. Lin. Coercivity enhancement near blocking temperature in exchange biased $\mathrm{Fe} / \mathrm{Fe}_{x} \mathrm{Mn}_{1-x}$ films on $\mathrm{Cu}(001)$. Journal of Applied Physics, 95(11):7297-7299, 2004.

[347] K. R. Nikolaev, A. Yu. Dobin, I. N. Krivorotov, W. K. Cooley, A. Bhattacharya, A. L. Kobrinskii, L. I. Glazman, R. M. Wentzovitch, E. Dan Dahlberg, and A. M. Goldman. Oscillatory Exchange Coupling and Positive Magnetoresistance in Epitaxial Oxide Heterostructures. Physical Review Letters, 85(17):3728-3731, 2000.

[348] P. Gaunt. Ferromagnetic domain wall pinning by a random array of inhomogeneities. Philosophical Magazine B, 48(3):261-276, 1983.

[349] D. S. Bethune, R. W. Smith, and Y. R. Shen. Sum-frequency generation via a resonant quadrupole transition in sodium. Physical Review A, 17(1):277-292, 1978.

[350] M. N. Iliev and M. V. Abrashev. Raman phonons and Raman JahnTeller bands in perovskite-like manganites. Journal of Raman Spectroscopy, 32(10):805-811, 2001.

[351] K.-Y. Choi, P. Lemmens, T. Sahaoui, G. Güntherodt, Yu. G. Pashkevich, V. P. Gnezdilov, P. Reutler, L. Pinsard-Gaudart, B. Büchner, and A. Revcolevschi. Existence of orbital polarons in ferromagnetic insulating 
$\mathrm{La}_{1-x} \mathrm{Sr}_{x} \mathrm{MnO}_{3}(0.11 \leqslant \mathrm{x} \leqslant 0.14)$ revealed by giant phonon softening. Physical Review B, 71(17), 2005.

[352] L. Brey. Electronic phase separation in manganite-insulator interfaces. Physical Review B, 75(10), 2007.

[353] K. Gehrke, V. Moshnyaga, K. Samwer, O. I. Lebedev, J. Verbeeck, D. Kirilenko, and G. Van Tendeloo. Interface controlled electronic variations in correlated heterostructures. Physical Review B, 82(11), 2010.

[354] G. C. Milward, M. J. Calderón, and P. B. Littlewood. Electronically soft phases in manganites. Nature, 433(7026):607-610, 2005.

[355] M. S. Rzchowski and R. Joynt. Electronic inhomogeneity at magnetic domain walls in strongly correlated systems. Europhysics Letters (EPL), 67(2):287293, 2004.

[356] K. H. Ahn, T. Lookman, and A. R. Bishop. Strain-induced metal-insulator phase coexistence in perovskite manganites. Nature, 428(6981):401-404, 2004.

[357] A. L. Kobrinskii, A. M. Goldman, Maria Varela, and S. J. Pennycook. Thickness dependence of the exchange bias in epitaxial manganite bilayers. Physical Review B, 79(9), 2009.

[358] Yu Lu, X. W. Li, G. Q. Gong, Gang Xiao, A. Gupta, P. Lecoeur, J. Z. Sun, Y. Y. Wang, and V. P. Dravid. Large magnetotunneling effect at low magnetic fields in micrometer-scale epitaxial $\mathrm{La}_{0.7} \mathrm{Sr}_{0.3} \mathrm{MnO}_{3}$ tunnel junctions. Physical Review B, 54(12):R8357-R8360, 1996.

[359] A. Dubey and V. G. Sathe. The effect of magnetic order and thickness in the Raman spectra of oriented thin films of $\mathrm{LaMnO}_{3}$. Journal of Physics: Condensed Matter, 19(34):346232, 2007.

[360] S. Merten, V. Roddatis, and V. Moshnyaga. Metalorganic-aerosol-deposited $\mathrm{Au}$ nanoparticles for the characterization of ultrathin films by SurfaceEnhanced Raman Spectroscopy. submitted to Applied Physics Letters, revised: 05.09.2019, 2019.

[361] J. Robertson. High dielectric constant gate oxides for metal oxide Si transistors. Reports on Progress in Physics, 69(2):327-396, 2006.

[362] Manuel Bibes and Agns Barthelemy. Oxide Spintronics. IEEE Transactions on Electron Devices, 54(5):1003-1023, 2007.

[363] S. Majumdar and S. van Dijken. Pulsed laser deposition of La1 $-x \mathrm{Sr}_{x} \mathrm{MnO}_{3}$ : thin-film properties and spintronic applications. Journal of Physics D: Applied Physics, 47(3):034010, 2014. 
[364] Manuel Bibes, Javier E. Villegas, and Agnès Barthélémy. Ultrathin oxide films and interfaces for electronics and spintronics. Advances in Physics, 60(1):5-84, 2011.

[365] M. Birkholz. Thin Film Analysis by X-Ray Scattering. Wiley, 2005.

[366] D. Hesse, S. K. Lee, and U. Gösele. Microstructure of (104)-oriented $\mathrm{Bi}_{3.25} \mathrm{La}_{0.75} \mathrm{Ti}_{3} \mathrm{O}_{12}$ and $\mathrm{Bi}_{3.54} \mathrm{Nd}_{0.46} \mathrm{Ti}_{3} \mathrm{O}_{12}$ ferroelectric thin films on multiply twinned $\mathrm{SrRuO}_{3} / \mathrm{Pt}(111)$ electrodes on YSZ(100)-buffered $\mathrm{Si}(100)$. physica status solidi (a), 202(12):2287-2298, 2005.

[367] P. Zaumseil. High-resolution characterization of the forbidden Si 200 and Si 222 reflections. Journal of Applied Crystallography, 48(Pt 2):528-532, 2015.

[368] E. Rossmanith. Approximate calculation of multiple-diffraction patterns based on Renninger's kinematical 'simplest approach'. Journal of Applied Crystallography, 33(3):921-927, 2000.

[369] C. G. Granqvist and R. A. Buhrman. Ultrafine metal particles. Journal of Applied Physics, 47(5):2200-2219, 1976.

[370] E. N. Aybeke, Y. Lacroute, C. Elie-Caille, A. Bouhelier, E. Bourillot, and E. Lesniewska. Homogeneous large-scale crystalline nanoparticle-covered substrate with high SERS performance. Nanotechnology, 26(24):245302, 2015.

[371] W. Ma, Z. Lu, and M. Zhang. Investigation of structural transformations in nanophase titanium dioxide by Raman spectroscopy. Applied Physics A: Materials Science \& Processing, 66(6):621-627, 1998.

[372] J. K. Burdett, T. Hughbanks, G. J. Miller, J. W. Richardson, and J. V. Smith. Structural-electronic relationships in inorganic solids: powder neutron diffraction studies of the rutile and anatase polymorphs of titanium dioxide at 15 and 295 K. Journal of the American Chemical Society, 109(12):3639-3646, 1987.

[373] R. M. Stöckle, V. Deckert, C. Fokas, and R. Zenobi. Controlled Formation of Isolated Silver Islands for Surface-Enhanced Raman Scattering. Applied Spectroscopy, 54(11):1577-1583, 2000.

[374] M. E. Hankus, D. N. Stratis-Cullum, and P. M. Pellegrino. Surface enhanced Raman scattering (SERS)-based next generation commercially available substrate: physical characterization and biological application. In Hooman Mohseni, Massoud H. Agahi, and Manijeh Razeghi, editors, Biosensing and Nanomedicine IV, SPIE Proceedings, page 80990N. SPIE, 2011.

[375] J. Jiang, Z. Xu, A. Ameen, F. Ding, G. Lin, and G. L. Liu. Large-area, lithography-free, low-cost SERS sensor with good flexibility and high performance. Nanotechnology, 27(38):385205, 2016. 
[376] W. F. Zhang, Y. L. He, M. S. Zhang, Z. Yin, and Q. Chen. Raman scattering study on anatase $\mathrm{TiO}_{2}$ nanocrystals. Journal of Physics D: Applied Physics, 33(8):912-916, 2000.

[377] F. Tian, Y. Zhang, J. Zhang, and C. Pan. Raman Spectroscopy: A New Approach to Measure the Percentage of Anatase $\mathrm{TiO}_{2}$ Exposed (001) Facets. The Journal of Physical Chemistry C, 116(13):7515-7519, 2012.

[378] M. Lazzeri, A. Vittadini, and A. Selloni. Structure and energetics of stoichiometric $\mathrm{TiO}_{2}$ anatase surfaces. Physical Review B, 63(15), 2001.

[379] W. E. Pickett and D. J. Singh. Transport and fermiology of the ferromagnetic phase of $\mathrm{La}_{0.67} \mathrm{~A}_{0.33} \mathrm{MnO}_{3}(\mathrm{~A}=\mathrm{Ca}, \mathrm{Sr}, \mathrm{Ba})$. Journal of Magnetism and Magnetic Materials, 172(3):237-246, 1997.

[380] C. H. Kim, G. Qi, K. Dahlberg, and W. Li. Strontium-Doped Perovskites Rival Platinum Catalysts for Treating $\mathrm{NO}_{x}$ in Simulated Diesel Exhaust. Science, 327(5973):1624-1627, 2010.

[381] M. Viret, J. Nassar, M. Drouet, J. P. Contour, C. Fermon, and A. Fert. Spin polarised tunnelling as a probe of half metallic ferromagnetism in mixedvalence manganites. Journal of Magnetism and Magnetic Materials, 198199:1-5, 1999.

[382] J. Suntivich, H. A. Gasteiger, N. Yabuuchi, H. Nakanishi, J. B. Goodenough, and Y. Shao-Horn. Design principles for oxygen-reduction activity on perovskite oxide catalysts for fuel cells and metal-air batteries. Nature Chemistry, 3(7):546-550, 2011.

[383] A. Filippetti and W. E. Pickett. Double-exchange-driven spin pairing at the (001) surface of manganites. Physical Review B, 62(17):11571-11575, 2000.

[384] J. W. Freeland, K. E. Gray, L. Ozyuzer, P. Berghuis, Elvira Badica, J. Kavich, H. Zheng, and J. F. Mitchell. Full bulk spin polarization and intrinsic tunnel barriers at the surface of layered manganites. Nature Materials, 4(1):62-67, 2004 .

[385] M. Talati and P. K. Jha. Phonons and Jahn-Teller distortion in manganites. Journal of Molecular Structure, 838(1-3):227-231, 2007.

[386] L. Yang, M. Gong, X. Jiang, D. Yin, X. Qin, B. Zhao, and W. Ruan. Investigation on SERS of different phase structure $\mathrm{TiO}_{2}$ nanoparticles. Journal of Raman Spectroscopy, 46(3):287-292, 2015.

[387] O. Prakash, S. Kumar, P. Singh, V. Deckert, S. Chatterjee, A. K. Ghosh, and R. K. Singh. Surface-enhanced Raman scattering characteristics of $\mathrm{CuO}: \mathrm{Mn} / \mathrm{Ag}$ heterojunction probed by methyl orange: effect of $\mathrm{Mn}^{2+}$ doping. Journal of Raman Spectroscopy, 47(7):813-818, 2016. 
[388] D. Petti, A. Stroppa, S. Picozzi, S. Brivio, M. Cantoni, and R. Bertacco. Effect of Au proximity on the LSMO surface: An ab initio study. Journal of Magnetism and Magnetic Materials, 324(17):2659-2663, 2012.

[389] D. Petti, R. Bertacco, S. Brivio, M. Cantoni, A. Cattoni, and F. Ciccacci. X-ray photoemission study of the $\mathrm{Au} / \mathrm{La}_{0.67} \mathrm{Sr}_{0.33} \mathrm{MnO}_{3}$ interface formation. Journal of Applied Physics, 103(4):044903, 2008.

[390] K. Huang, W. Pan, J. F. Zhu, J. C. Li, N. Gao, C. Liu, L. Ji, E. T. Yu, and J. Y. Kang. Asymmetric light reflectance from metal nanoparticle arrays on dielectric surfaces. Scientific Reports, 5(1), 2015.

[391] P. Dore, P. Postorino, A. Sacchetti, M. Baldini, R. Giambelluca, M. Angeloni, and G. Balestrino. Raman measurements on thin films of the $\mathrm{La}_{0.7} \mathrm{Sr}_{0.3} \mathrm{MnO}_{3}$ manganite: a probe of substrate-induced effects. The European Physical Journal B, 48(2):255-258, 2005.

[392] J. Kreisel, G. Lucazeau, C. Dubourdieu, M. Rosina, and F. Weiss. Raman scattering study of $\mathrm{La}_{0.7} \mathrm{Sr}_{0.3} \mathrm{MnO}_{3} / \mathrm{SrTiO}_{3}$ multilayers. Journal of Physics: Condensed Matter, 14(20):5201-5210, 2002.

[393] P. Björnsson, M. Rübhausen, J. Bäckström, M. Käll, S. Eriksson, J. Eriksen, and L. Börjesson. Lattice and charge excitations in $\mathrm{La}_{1-x} \mathrm{Sr}_{x} \mathrm{MnO}_{3}$. Physical Review B, 61(2):1193-1197, 2000.

[394] A.-M. Haghiri-Gosnet and J.-P. Renard. CMR manganites: physics, thin films and devices. Journal of Physics D: Applied Physics, 36(8):R127-R150, 2003.

[395] S. Valencia, L. Peña, Z. Konstantinovic, Ll Balcells, R. Galceran, D. Schmitz, F. Sandiumenge, M. Casanove, and B. Martínez. Intrinsic antiferromagnetic/insulating phase at manganite surfaces and interfaces. Journal of Physics: Condensed Matter, 26(16):166001, 2014.

[396] M. V. Abrashev, J. Bäckström, L. Börjesson, V. N. Popov, R. A. Chakalov, N. Kolev, R.-L. Meng, and M. N. Iliev. Raman spectroscopy of $\mathrm{CaMnO}_{3}$ : Mode assignment and relationship between Raman line intensities and structural distortions. Physical Review B, 65(18), 2002.

[397] J. M. Zuo and J. Tao. Nanometer-sized regions of charge ordering and charge melting in $\mathrm{La}_{0.67} \mathrm{Ca}_{0.33} \mathrm{MnO}_{3}$ revealed by electron microdiffraction. Physical Review B, 63(6), 2001.

[398] M. Pissas and G. Kallias. Phase diagram of the $\mathrm{La}_{1-x} \mathrm{Ca}_{x} \mathrm{MnO}_{3}$ compound $(0.5<x<0.9)$. Physical Review B, 68(13), 2003. 
[399] Y. Tomioka, A. Asamitsu, H. Kuwahara, Y. Moritomo, and Y. Tokura. Magnetic-field-induced metal-insulator phenomena $\mathrm{La}_{1-x} \mathrm{Ca}_{x} \mathrm{MnO}_{3}$ with controlled charge-ordering instability. Physical Review B, 53(4):R1689-R1692, 1996.

[400] D. Louca, T. Egami, E. L. Brosha, H. Röder, and A. R. Bishop. Local JahnTeller distortion in La1 $-x \mathrm{Sr}_{x} \mathrm{MnO}_{3}$ observed by pulsed neutron diffraction. Physical Review B, 56(14):R8475-R8478, 1997.

[401] S. Merten, V. Bruchmann-Bamberg, B. Damaschke, K. Samwer, and V. Moshnyaga. Jahn-Teller reconstructed surface of the doped manganites shown by means of surface-enhanced Raman spectroscopy. Physical Review Materials, 3(6), 2019.

[402] A. E. Pantoja, H. J. Trodahl, R. G. Buckley, Y. Tomioka, and Y. Tokura. Raman spectroscopy of orthorhombic $\mathrm{La}_{1-x} \mathrm{Ca}_{x} \mathrm{MnO}_{3}, x=0.1-0.3$. Journal of Physics: Condensed Matter, 13(16):3741-3752, 2001.

[403] S. Valencia, A. Gaupp, W. Gudat, Ll. Abad, Ll. Balcells, and B. Martínez. Surface degradation of magnetic properties in manganite thin films proved with magneto-optical techniques in reflection geometry. Applied Physics Letters, 90(25):252509, 2007.

[404] M. Bowen, M. Bibes, A. Barthelemy, J.-P. Contour, A. Anane, Y. Lemaitre, and A. Fert. Nearly total spin polarization in $\mathrm{La}_{0.67} \mathrm{Sr}_{0.33} \mathrm{MnO}_{3}$ from tunneling experiments. Applied Physics Letters, 82(2):233-235, 2003.

[405] J.-O. Krisponeit, C. Kalkert, B. Damaschke, V. Moshnyaga, and K. Samwer. Time-resolved resistive switching on manganite surfaces: Creep and $1 / \mathrm{f}^{\text {alpha }}$ noise signatures indicate pinning of nanoscale domains. Physical Review B, 87(12), 2013.

[406] H. Fujishiro, T. Fukase, and M. Ikebe. Charge Ordering and Sound Velocity Anomaly in $\mathrm{La}_{1-x} \mathrm{Sr}_{x} \mathrm{MnO}_{x \geq 0.5}$. Journal of the Physical Society of Japan, 67(8):2582-2585, 1998.

[407] J. Hemberger, A. Krimmel, T. Kurz, H.-A. Krug von Nidda, V. Yu. Ivanov, A. A. Mukhin, A. M. Balbashov, and A. Loidl. Structural, magnetic, and electrical properties of single-crystalline $\mathrm{La}_{1-x} \mathrm{Sr}_{x} \mathrm{MnO}_{3}(0.4<\mathrm{x}<0.85)$. Physical Review B, 66(9), 2002.

[408] O. Chmaissem, B. Dabrowski, S. Kolesnik, J. Mais, J. D. Jorgensen, and S. Short. Structural and magnetic phase diagrams of $\mathrm{La}_{1-x} \mathrm{Sr}_{x} \mathrm{MnO}_{3}$ and $\mathrm{Pr}_{1-y} \mathrm{Sr}_{y} \mathrm{MnO}_{3}$. Physical Review B, 67(9), 2003.

[409] J. J. Kavich, M. P. Warusawithana, J. W. Freeland, P. Ryan, X. Zhai, R. H. Kodama, and J. N. Eckstein. Nanoscale suppression of magnetization at 
atomically assembled manganite interfaces: XMCD and XRMS measurements. Physical Review B, 76(1), 2007.

[410] A. Belenchuk, O. Shapoval, V. Roddatis, V. Bruchmann-Bamberg, K. Samwer, and V. Moshnyaga. Ruddlesden-Popper interface in correlated manganite heterostructures induces magnetic decoupling and dead layer reduction. Applied Physics Letters, 109(23):232405, 2016.

[411] J. J. Peng, C. Song, F. Li, Y. D. Gu, G. Y. Wang, and F. Pan. Restoring the magnetism of ultrathin $\mathrm{LaMnO}_{3}$ films by surface symmetry engineering. Physical Review B, 94(21), 2016.

[412] S. Mahajan, R. M. Cole, J. D. Speed, S. H. Pelfrey, A. E. Russell, P. N. Bartlett, S. M. Barnett, and J. J. Baumberg. Understanding the SurfaceEnhanced Raman Spectroscopy "Background". The Journal of Physical Chemistry $C$, 114(16):7242-7250, 2009.

[413] R. Gautam, S. Vanga, F. Ariese, and S. Umapathy. Review of multidimensional data processing approaches for Raman and infrared spectroscopy. EPJ Techniques and Instrumentation, 2(1), 2015.

[414] H. M. Manasevit and W. I. Simpson. Single-Crystal Silicon on a Sapphire Substrate. Journal of Applied Physics, 35(4):1349-1351, 1964.

[415] S. Cristoloveanu. Silicon on insulator technologies and devices: from present to future. Solid-State Electronics, 45(8):1403-1411, 2001.

[416] C. Kalkert, J.-O. Krisponeit, M. Esseling, O. I. Lebedev, V. Moshnyaga, B. Damaschke, G. van Tendeloo, and K. Samwer. Resistive switching at manganite/manganite interfaces. Applied Physics Letters, 99(13):132512, 2011.

[417] V. Bhosle and J. Narayan. Epitaxial growth and magnetic properties of $\mathrm{La}_{0.7} \mathrm{Sr}_{0.3} \mathrm{MnO}_{3}$ films on (0001) sapphire. Applied Physics Letters, 90(10):101903, 2007.

[418] H. L. Ju, Kannan M. Krishnan, and D. Lederman. Evolution of straindependent transport properties in ultrathin $\mathrm{La}_{0.67} \mathrm{Sr}_{0.33} \mathrm{MnO}_{3}$ films. Journal of Applied Physics, 83(11):7073-7075, 1998.

[419] M. Lucht, M. Lerche, H.-C. Wille, Yu. V. Shvyd'ko, H. D. Rüter, E. Gerdau, and P. Becker. Precise measurement of the lattice parameters of $\alpha$-Al2O3in the temperature range 4.5-250 $\mathrm{K}$ using the Mössbauer wavelength standard. Journal of Applied Crystallography, 36(4):1075-1081, 2003. 


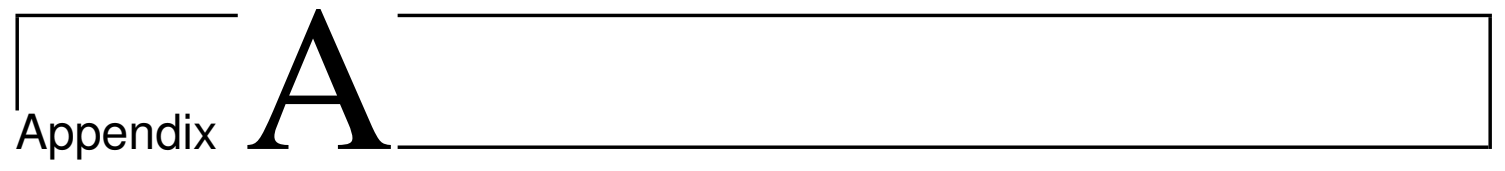

\section{Supplementary Information for Chapter 4}

Herewith, all additional information regarding the characterization of the LPCMO film, the evaluation of the Raman spectra, deduction of the $T-H$ phase diagram and the experimental setup are provided. This appendix represents the supplemental material to the publication presented in $\mathrm{Ch} 4$

\section{S1. Structural, electrical and magnetic characterization}

$\mathrm{X}$-ray diffraction (XRD) and X-ray reflectometry (XRR) structural characterization of our LPCMO film demonstrates an out-of-plane epitaxial grown film on the $\mathrm{MgO}(100)$ substrate with a pseudo-cubic lattice constant, $a=3.871 \AA$, and a thickness, $d=66 \mathrm{~nm}$. Electric and magnetic measurements display coupled insulatormetal and paramagnetic-ferromagnetic transitions with a transition temperature $T_{\mathrm{MI}} \approx T_{\mathrm{C}} \approx 197 \mathrm{~K}$ (see Fig, A.1). In Fig, A.1, we present the $R(T)$ curves for different applied magnetic fields, $H=0 \mathrm{kOe}, 10 \mathrm{kOe}, 30 \mathrm{kOe}$ and $50 \mathrm{kOe}$.

\section{S2. Details of the evaluation of the Raman spectra of LPCMO}

First, the Raman spectra were corrected by the Bose-Einstein thermal factor $(1+$ $n(\omega))=\left[1-\exp \left(-\hbar \omega / k_{\mathrm{B}} T\right)\right]^{-1}$. The electronic background continuum was modeled by a collision-limited model $R_{\mathrm{el}}=B G \omega /\left(G^{2}+\omega^{2}\right)$ with $B, G$ and $\omega$ as intensity, linewidth and frequency, respectively. The phonon peaks were modeled by the best fit of multiple Lorentzian line shapes. Exemplary fits for different temperatures and applied magnetic fields are shown in Fig. A.4 and A.5. Additional information concerning possible heating effects and the splitting of the JT mode are shown in Fig. A.3 and A.6.

\section{S3. Field alignment in the optical setup}

Figure A.7 shows a sketch of the optical experiment. The laser beam was focused onto the sample by using a 50xLWD objective with a working distance, 
$d_{\mathrm{WD}}=10.6 \mathrm{~mm}$. The sample was mounted on a $\mathrm{Cu}$ sample holder (thermal contact with silver paste at the corners of the sample), which is placed in the center of a superconducting solenoid magnetic coil inside a commercial cryostat (Microstat MO, Oxford Instruments) in vacuum $\left(10^{6}-10^{7} \mathrm{mbar}\right)$. The field homogeneity is below $2 \%$ over a $5 \mathrm{~mm}$ diameter sphere with stability is $0.01 \% / \mathrm{h}$ [270]. The field can be considered as homogenous inside the probed sample area, since the Raman spectrometer only probes a small area of the sample $(<2 \mu \mathrm{m})$.

\section{S4. Deduction of the $T-H$ phase diagram from the $M(H)$ curves}

To create the $T$ - $H$ phase diagram, we measured the $M(H)$ curves and reconstructed the $M(T)$ curves by picking the magnetization value from the $M(H)$ curve at different magnetic fields at a specific temperature. The obtained $\mathrm{M}(\mathrm{T})$ curves are shown in Fig A.8. The $T_{\mathrm{C}}(H)$ value of the $T-H$ phase diagram is defined by the linear approximation of the $M(T)$ curve to $M=0 \mathrm{emu} / \mathrm{cm}^{3}$. The $T^{*}(H)$ is obtained from the field of a maximum of $\mathrm{d} M / \mathrm{d} H_{T>T c(0)}$. The $M(H)$ curves and the corresponding $\mathrm{d} M / \mathrm{d} H_{T>T c(0)}$ curves are shown in Fig. A.8 b-c as well as in Fig.4.4a in the main manuscript. 

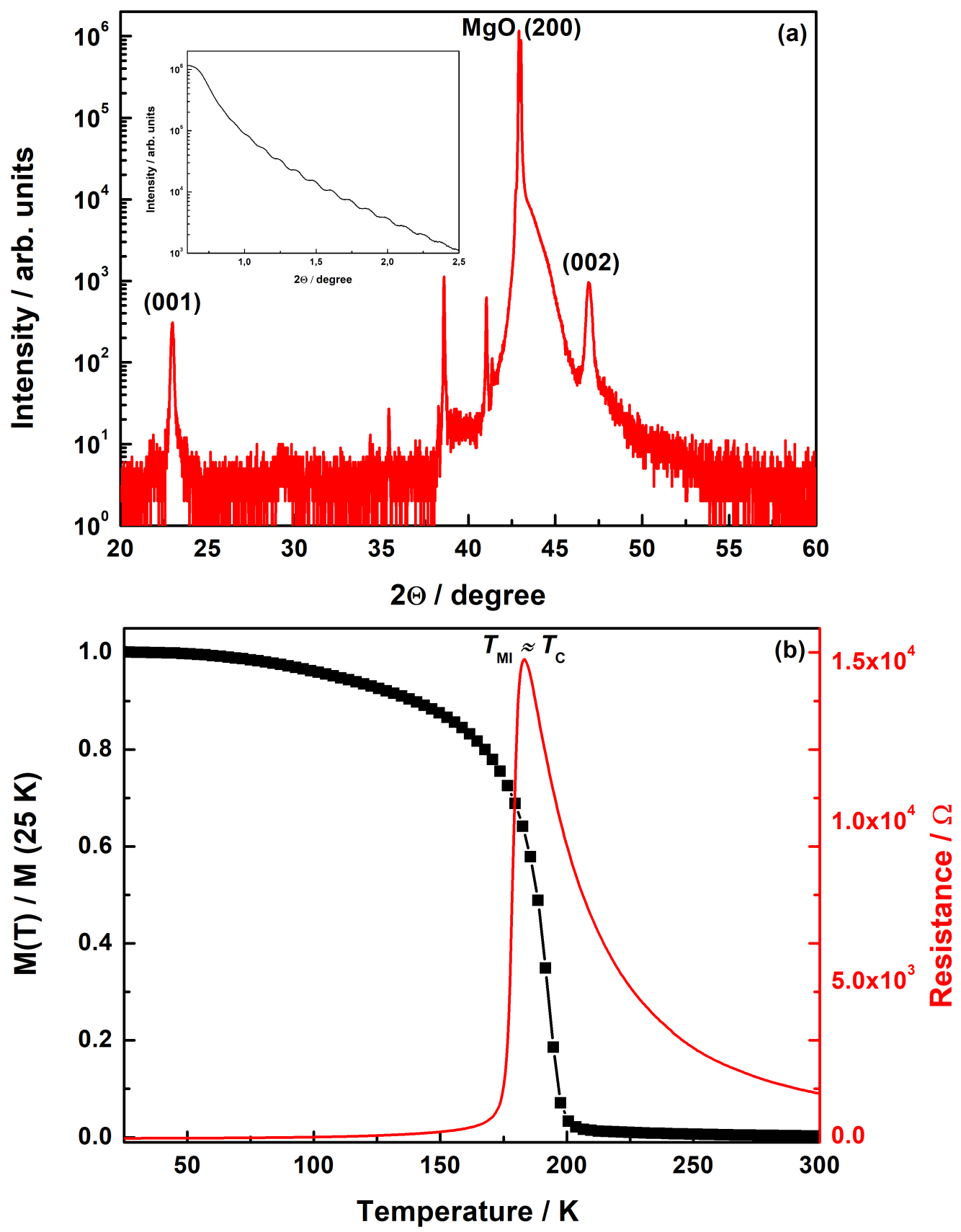

Figure A.1.: (a) XRD and XRR pattern and (b) $R(T)$ and $M(T)$ curves of the thin LPCMO film. The LPCMO film shows out-of-plane epitaxial growth with a pseudo-cubic lattice constant, $a=3.871 \AA$ and a transition temperature $T_{\mathrm{MI}} \approx T_{\mathrm{C}} \approx 197 \mathrm{~K}$. 


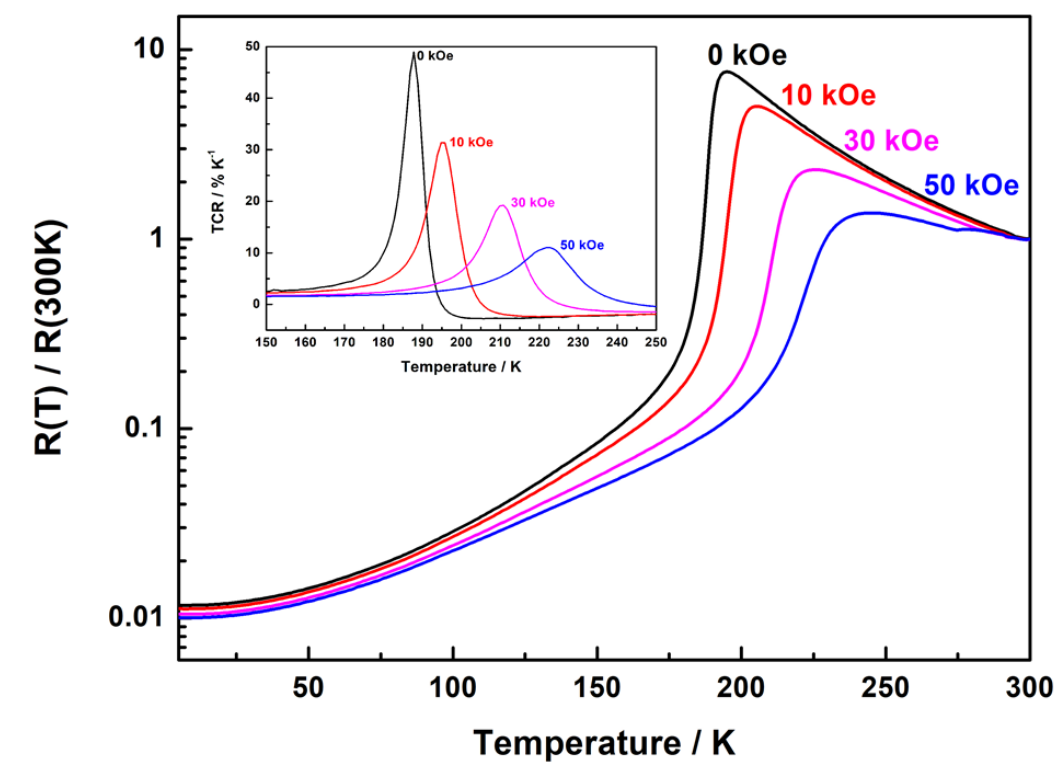

Figure A.2.: $R(T)$ for different applied magnetic fields, $H=0 \mathrm{kOe}, 10 \mathrm{kOe}, 30 \mathrm{kOe}$ and $50 \mathrm{kOe}$.

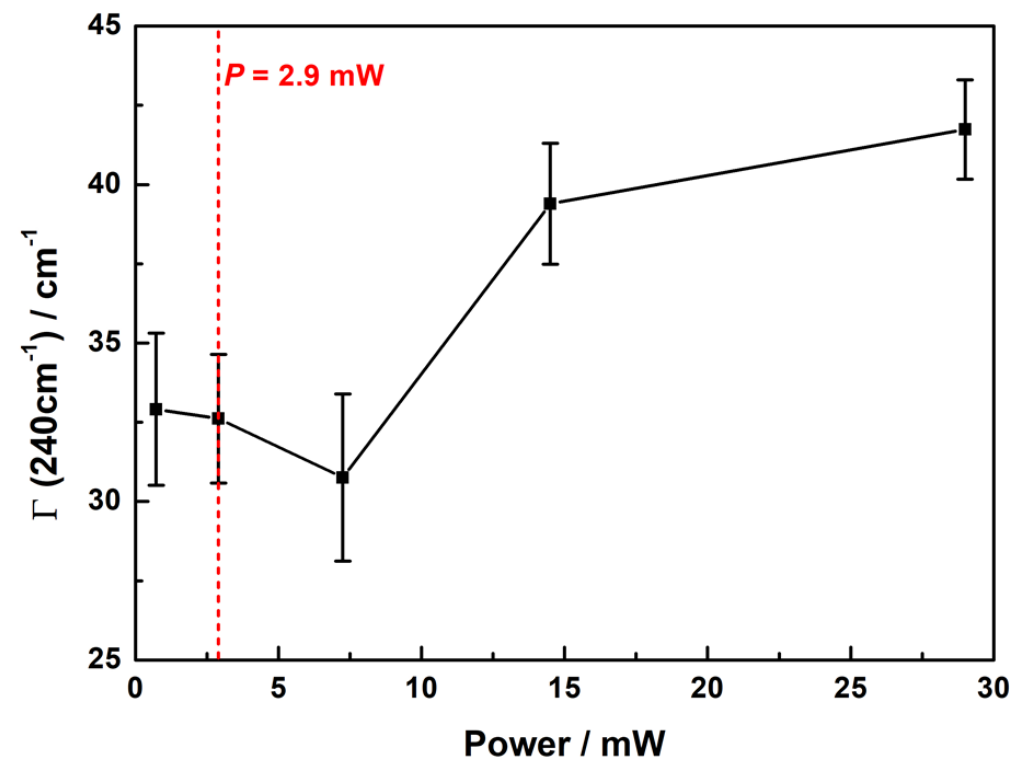

Figure A.3.: Power dependence of the linewidth of the $240 \mathrm{~cm}^{-1}$ mode at room temperature. For $P<7.5 \mathrm{~mW}$, the linewidth practically does not change, indicating no significant laser-induced heating of the film. 


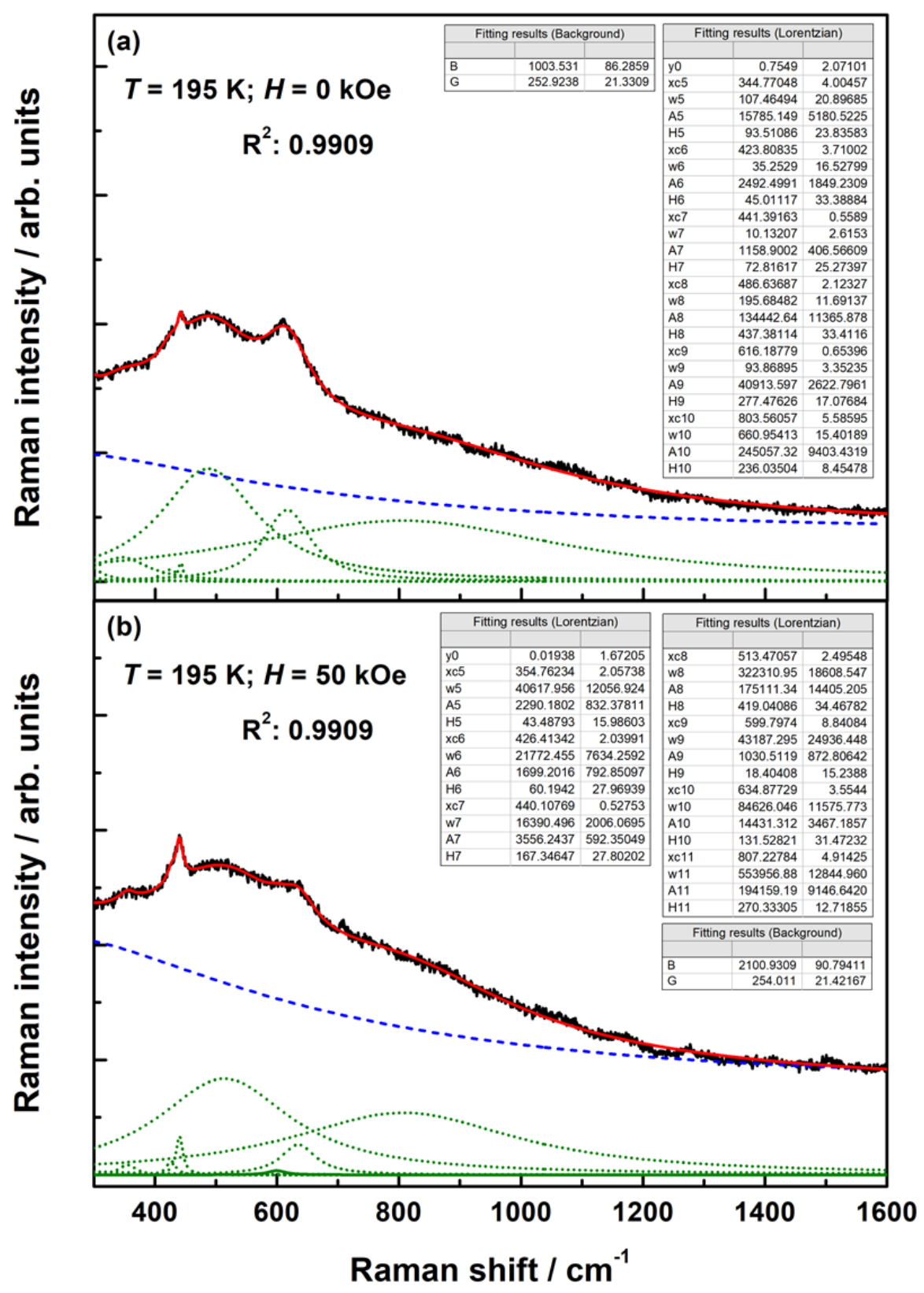

Figure A.4.: Raw Raman spectra (black), the subtracted baseline (dashed blue), the best fit of the Lorentzian line shapes (dotted green) and the resulting modeled Raman spectrum (red) of the LPCMO film for $H=0 \mathrm{kOe}$, $50 \mathrm{kOe}$ at $T=195 \mathrm{~K}$ in the region of interest $\left(300-1600 \mathrm{~cm}^{-1}\right)$ 

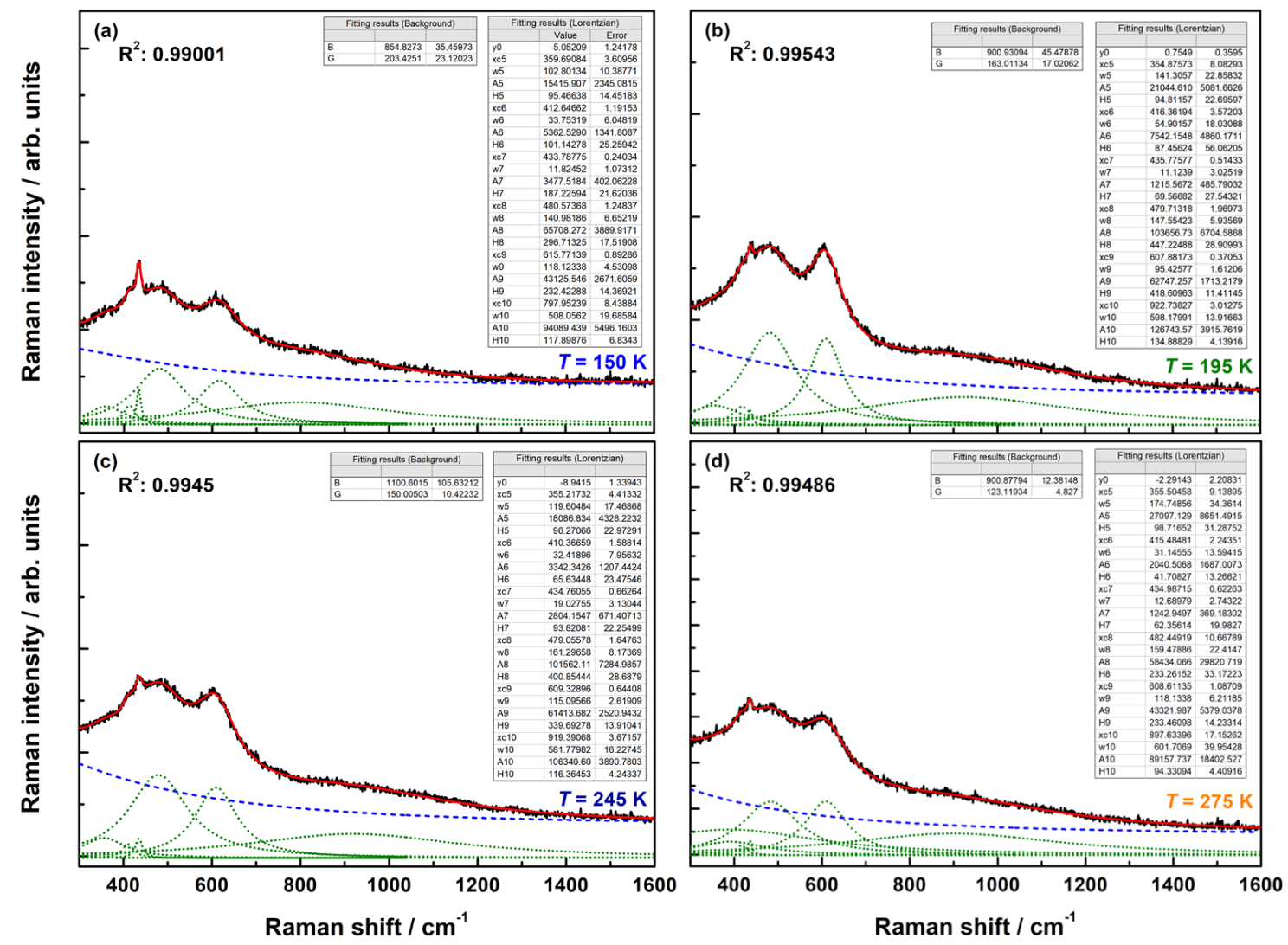

Figure A.5.: Best fit of Lorentzian line shapes of the Raman spectra for (a) $T=150 \mathrm{~K}$, (b) $T=195 \mathrm{~K}$, (c) $T=245 \mathrm{~K}$ and (d) $T=275 \mathrm{~K}$ in the region of interest $\left(300-900 \mathrm{~cm}^{-1}\right)$ for several temperatures of our temperature-dependent Raman study, presented in Fig 4.4 in the main manuscript. 


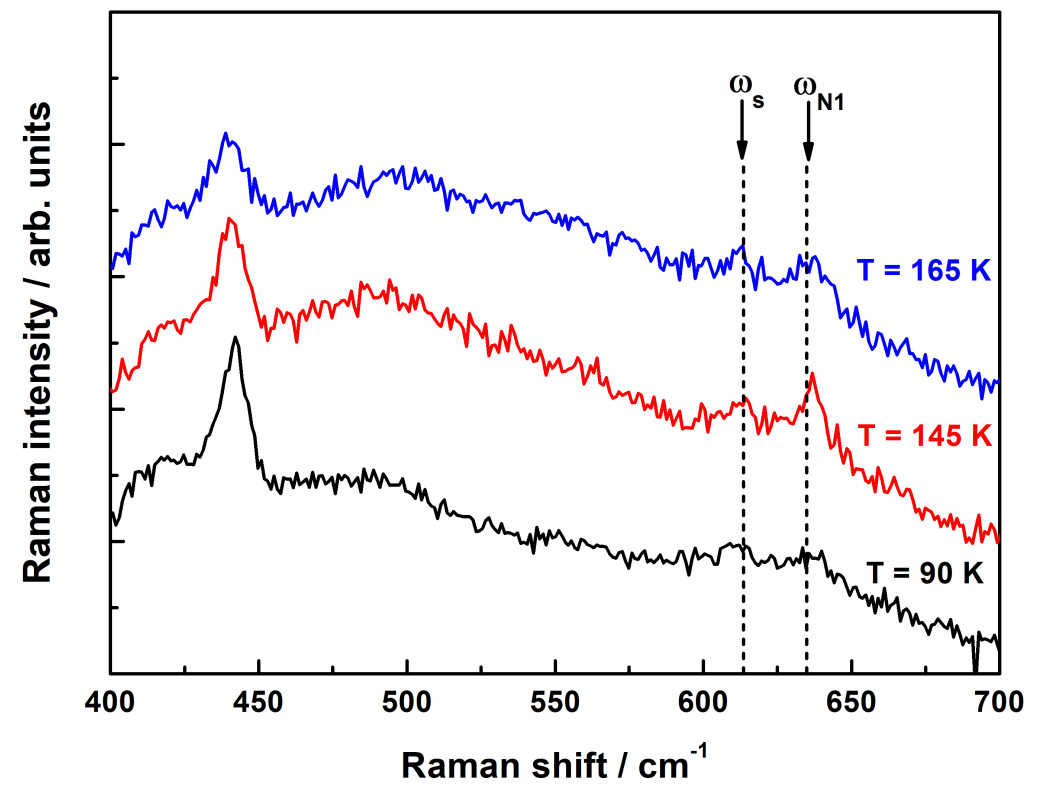

Figure A.6.: The splitting of the JT mode is also seen for $T<T_{\mathrm{C}}$ and an applied magnetic field, $H=50 \mathrm{kOe}$.

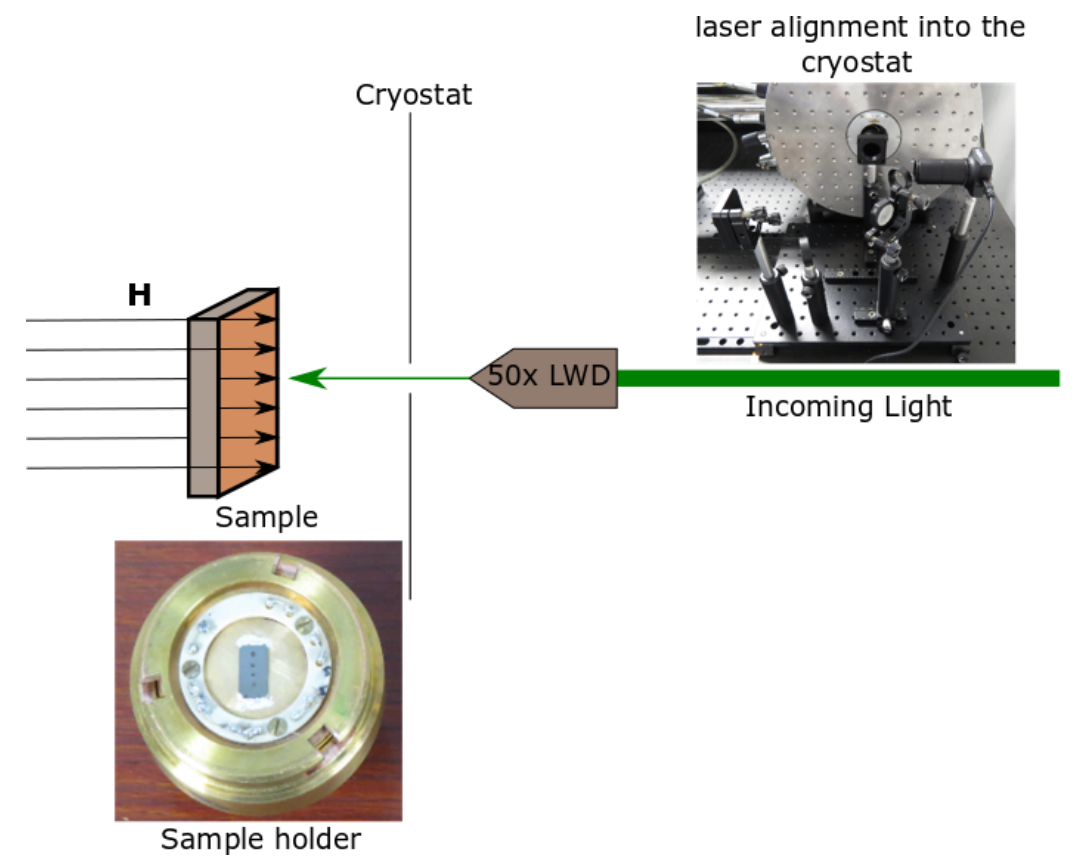

Figure A.7.: Sketch of the alignment of the optical experiment and the field alignment in the cryostat. 

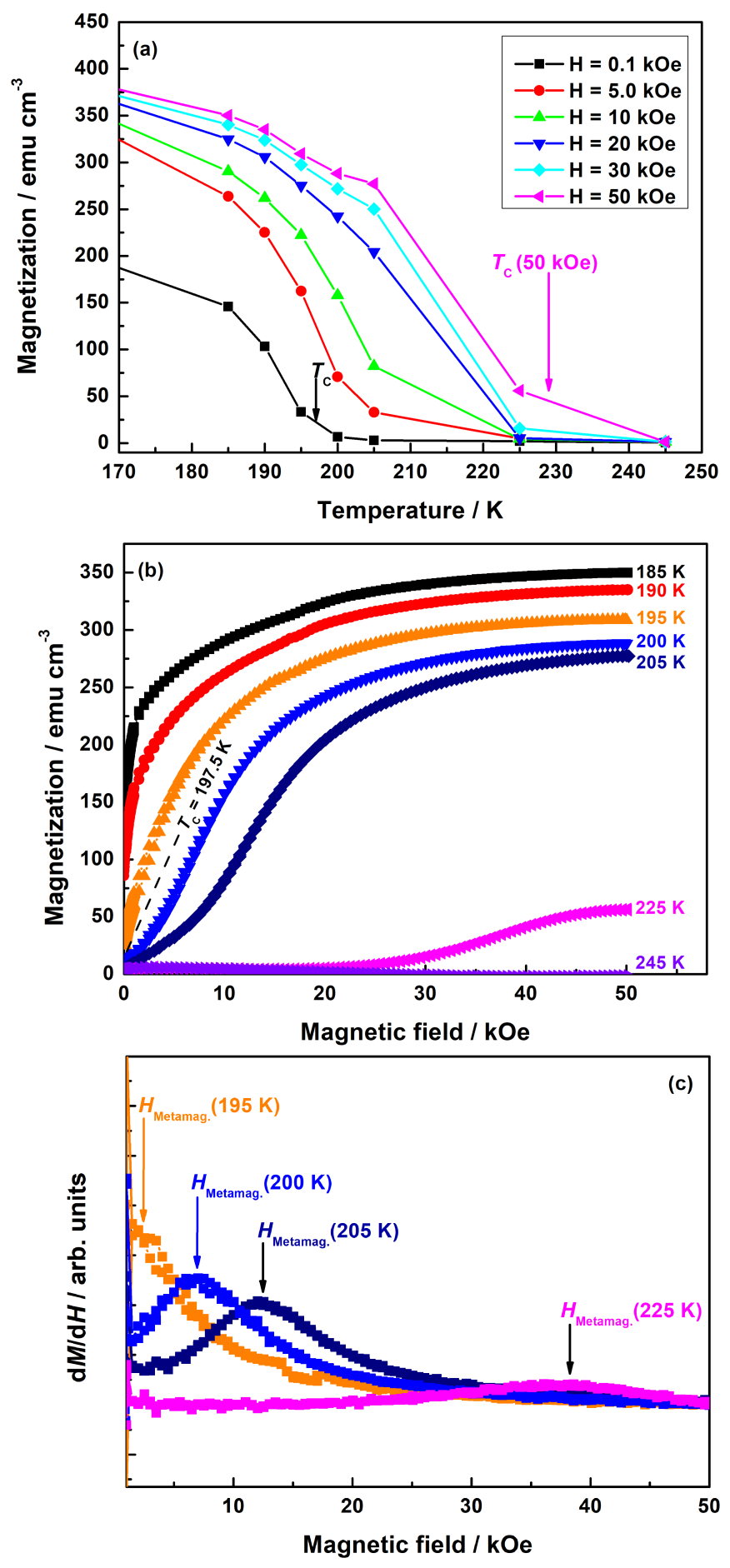

Figure A.8.: (a) The reconstructed $M(T)$ curves for applied magnetic fields $H=0.1$, 5, 10, 30 and $50 \mathrm{kOe}$. Exemplary, the derived transition temperatures $T_{\mathrm{C}}(H)$ for $H=0.1 \mathrm{kOe}$ and $H=50 \mathrm{kOe}$ are marked by arrows. (b) Exemplary $M(H)$ and (c) d $M / \mathrm{d} H_{T>T c(0)}$ curves, from which we obtained the temperature of the metamagnetic transition, $T^{*}(H)$. 
$\prod_{\text {Appendix }} \mathbf{D}$

\section{Supplementary Information for Chapter 6}

Herewith, all additional information regarding the characterization of the LCMO and LMSO films, the evaluation of the Raman spectra and a sketch of the experimental setup for the reflectance measurements are provided. This appendix represents the supplemental material to the publication presented in $\mathrm{Ch} .6$.

\section{SI.I. Thin Film Characterization of $\mathrm{La}_{0.7} \mathrm{Ca}_{0.3} \mathrm{MnO}_{3}, \mathrm{La}_{0.7} \mathrm{Sr}_{0.3} \mathrm{MnO}_{3}$ and $\mathrm{CaMnO}_{3}$}

This section provides further information about the properties of our thin manganite films before and after the deposition of Au nanoparticles (Au-NP). As mentioned in the main part of the paper, we observe no alteration of the manganite film after the $\mathrm{Au}-\mathrm{NP}$ deposition. Especially, the electrical and magnetic properties are the same, i.e. $R(T), M(T)$ and $M(H)$ curves are almost identical.

In Fig.B.1 B.4 the structural, electrical and magnetic properties of the LCMO and LSMO films are presented. X-Ray Diffraction (XRD) reveals an out-of-plane epitaxy on $\mathrm{MgO}(100)$ with pseudo-cubic lattice constants, $a_{\mathrm{LCMO}}=3.867 \AA$ and $a_{\mathrm{LSMO}}=3.882 \AA$, which are very close to the corresponding bulk values [99, 222] indicating a strain-free state of the manganite films on $\mathrm{MgO}(100)$. The transition temperature was determined as the temperature of the maximum/minimum of the temperature coefficient of resistivity and magnetization, $T C R(T)=1 / R \mathrm{~d} R(T) / \mathrm{d} T$ and $T C M(T)=1 / M \mathrm{~d} M(T) / \mathrm{d} T(T)$, shown in the insert of B.2 and B.3. One can see that the transition temperature does not change after the Au-NP deposition. Moreover, Fig. B.4 shows that deposition of Au-NPs also do not change the low temperature hysteresis curves $M(H)$. The obtained saturation magnetization, $M_{\text {Sat }}(\mathrm{LCMO})=3.61 \mu_{\mathrm{B}} / \mathrm{Mn}$ and $M_{\text {Sat }}(\mathrm{LSMO})=3.66 \mu_{\mathrm{B}} / \mathrm{Mn}$, is close to the theoretical bulk value, $M_{\mathrm{th}}=3.7 \mu_{\mathrm{B}} / \mathrm{Mn}$, for a manganite with the doping level, $x=0.3$. Additionally, the identical coercive field, $H_{c}=188$ Oe in LCMO and $H_{c}=67$ Oe in LSMO, before and after Au- deposition supports an intact bulk behavior. 
In Fig, B.5, the plasmonic characterization of the $\mathrm{CaMnO}_{3}$ film is shown. Reflectance measurements show a broad surface plasmon with its maximal resonance wavelength, $\lambda_{\mathrm{SPR}}=693 \mathrm{~nm}$. Although, the excitation wavelength $(\lambda=632.8 \mathrm{~nm})$ is much lower than the peak wavelength, the broad nature of the surface plasmon still gives rise to a plasmonic enhancement effect. Figure B.6 shows a comparison of the XRD pattern of an exemplary manganite film and the XRD pattern of the $\mathrm{MgO}$ substrate.

\section{SI.II. Details of the evaluation of the Raman spectra}

The raw SERS spectra are superimposed by an electronic continuum originating from different physical processes: electronic excitations in the manganite film itself [65], the background contribution due to the plasmonic enhancement [412] and the strong luminescence of the $\mathrm{MgO}$ substrate under excitation by laser light with $\lambda=633 \mathrm{~nm}$. Since the electronic continuum of the manganite itself is rather weak, the main contributions to the background continuum originate from the plasmonic enhancement and the luminescence of the $\mathrm{MgO}$ substrate.

First, we normalized the Raman spectra to the strongest $\mathrm{MgO}$ background peak and subtracted the Raman spectrum of a blank $\mathrm{MgO}$ substrate from it. Afterwards, we fitted the background continuum with a polynomial function of the $5^{\text {th }}$ grade (red line) [413] as depicted in Fig.B.7. Finally, we obtained the intensities from the best fit to multiple Lorentzian line shapes (see Fig.6.2 in the main part). Thicknessdependent Raman spectra of LSMO are shown in Fig.B.8

\section{SI.III. Experimental setup of the reflectance measurement}

The reflectance was measured under normal incidence using a halogen lamp as light source. The corresponding setup is shown in Fig.B.9. Lens L1 collimates the outcoming light, which is further focused onto the sample with a 50xLWD microscope objective $(\mathrm{NA}=0.25)$. Subsequently, the reflected light is coupled into the spectrometer via a beam splitter (BS) and two additional lenses. Since we measured the reflectance under normal incidence and the light propagates through a material with refractive index $r_{1}$ toward an interface consisting of gold nanoparticles against a medium with refractive index $r_{2}$ and the circumstance that $r_{2}$ (manganite) $>r_{1}$ (air), the surface plasmon resonance is represented by a maximum in the reflectance measurements [390]. 

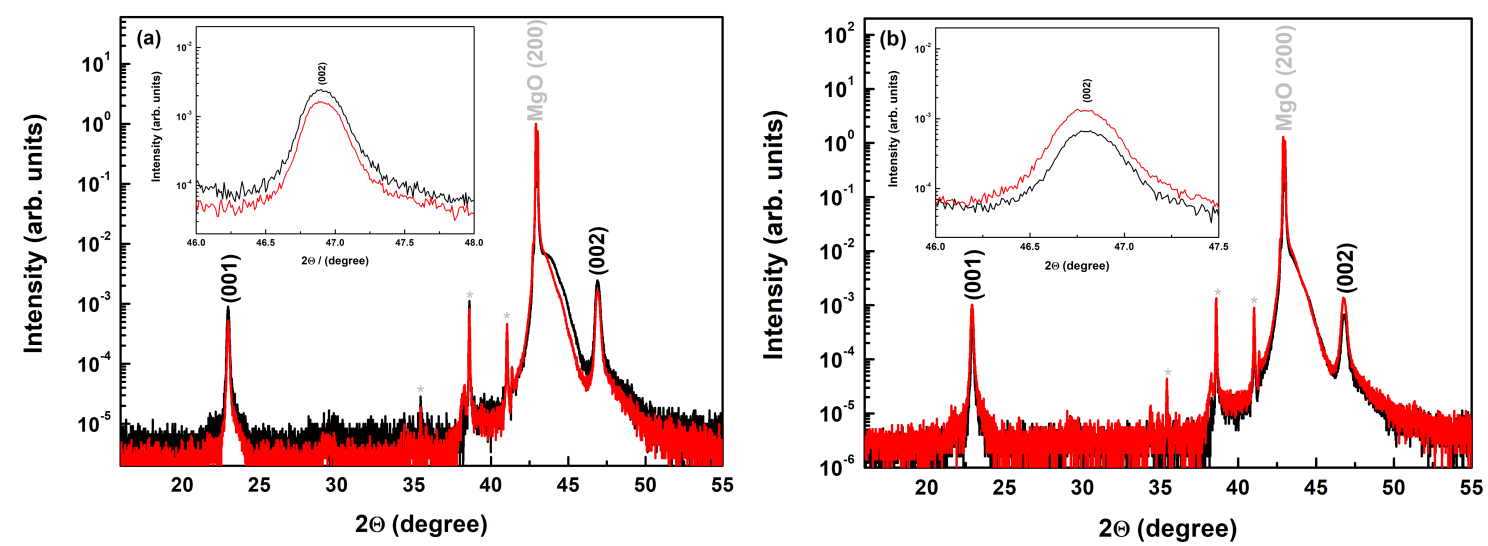

Figure B.1.: XRD patterns demonstrate an out-of-plane epitaxial growth of LCMO (left) and LSMO (right) films with thickness, $d=81 \mathrm{~nm}$ and $89 \mathrm{~nm}$, respectively. The pseudo-cubic lattice constants, $a_{\mathrm{LCMO}}=3.867 \AA$ (left) and $a_{\mathrm{LSMO}}=3.882 \AA$ (right), are very close to the corresponding bulk values, indicating a strain-free state of the manganite films on $\mathrm{MgO}(100)$. After the Au-NP deposition (red), the lattice constant shows no relaxation effects.
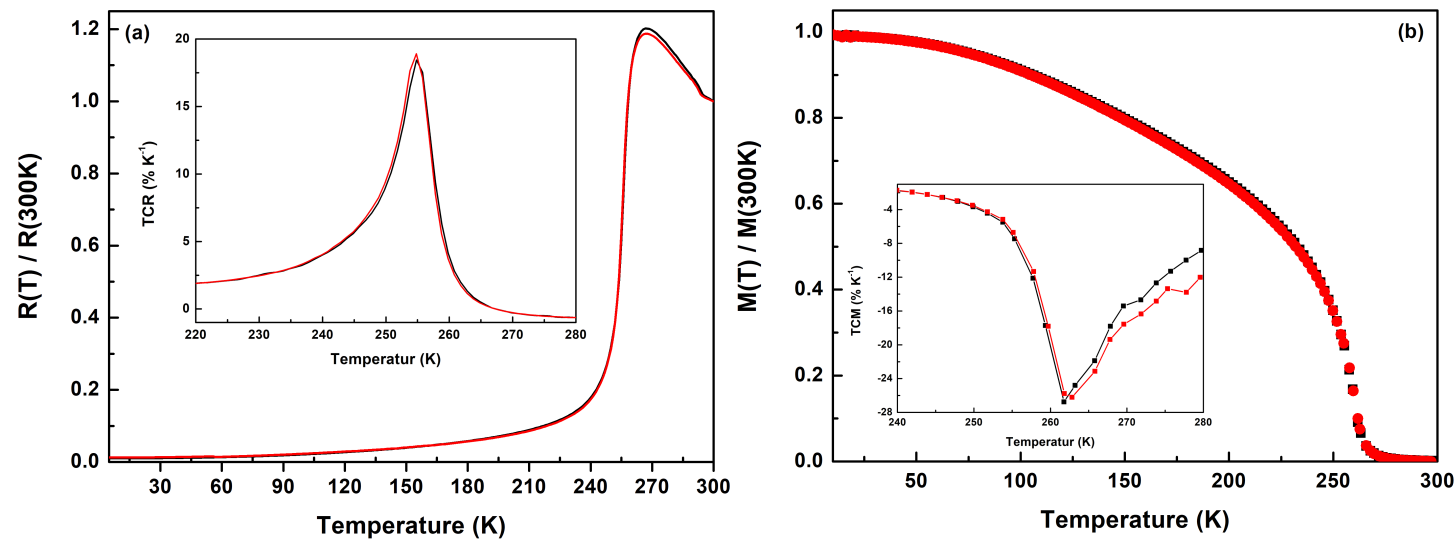

Figure B.2.: Electrical (left) and magnetic (right) characterization of the LCMO film demonstrates a metal-insulator coupled to a ferromagneticparamagnetic transition at $T_{\mathrm{MI}} \approx T_{\mathrm{C}} \approx 267 \mathrm{~K}$. The insert shows the TCR and TCM curves, before (black) and after (red) the deposition of $\mathrm{Au}-\mathrm{NPs}$. 

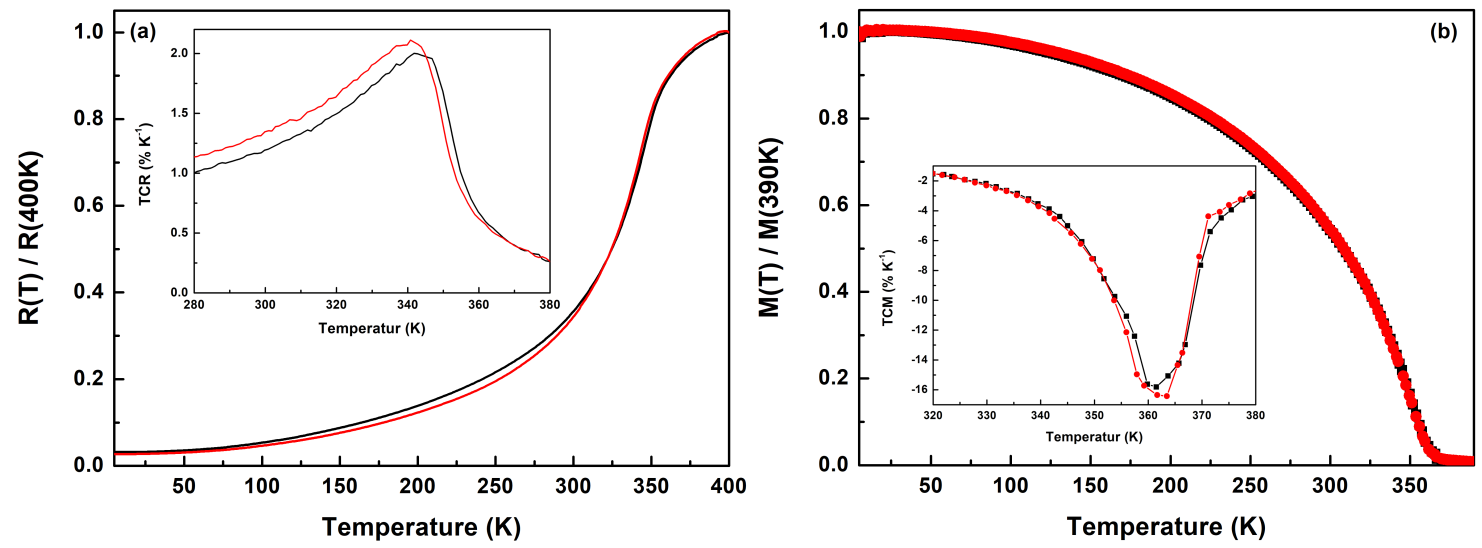

Figure B.3.: Electrical (left) and magnetic (right) characterization of the LSMO film show a metal-metal transition at $T_{\mathrm{MM}} \approx 343 \mathrm{~K}$ and a ferromagneticparamagnetic transition at $T_{\mathrm{C}} \approx 364 \mathrm{~K}$. As in the case of LCMO, the TCR and the TCM curves, before (black) and after (red) the Au-NP deposition, are almost identical and show negligible changes.
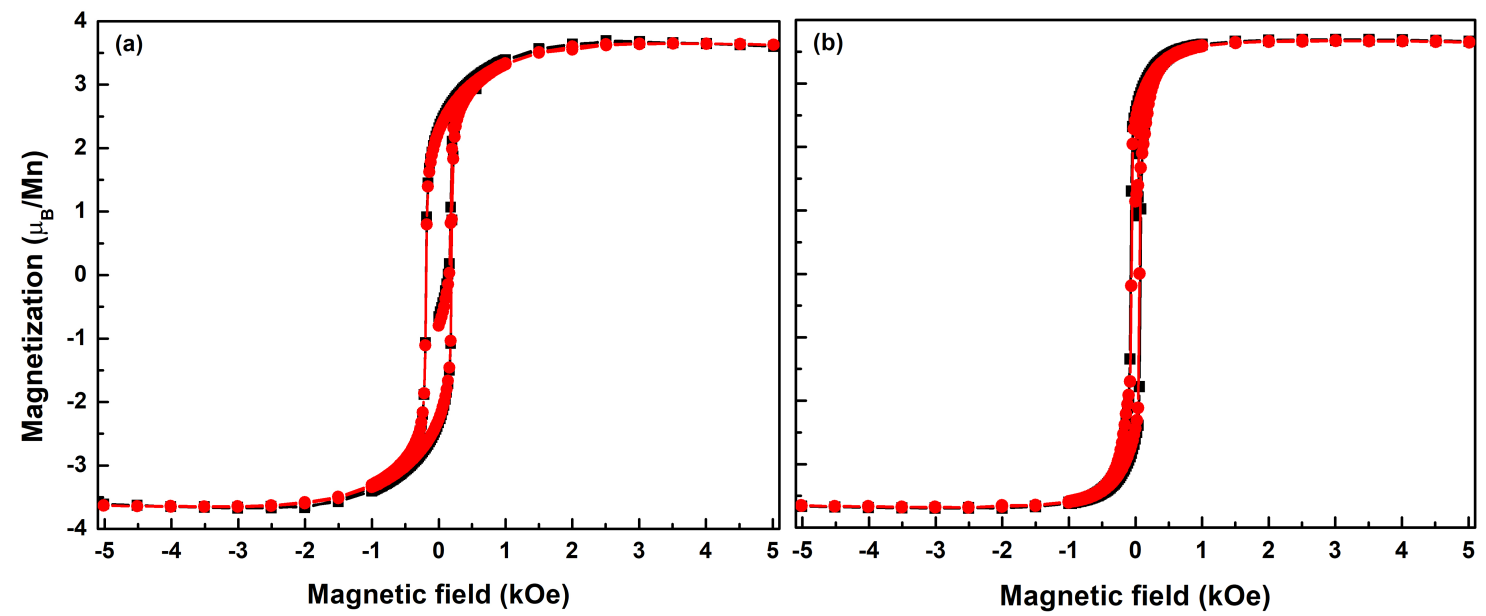

Figure B.4.: Low-temperature $\mathrm{M}(\mathrm{H})$ hysteresis curves $(T=5 \mathrm{~K})$ show a saturation magnetization $M_{\text {Sat }} \approx 3.61 \mu_{\mathrm{B}} / \mathrm{Mn}$ and a coercive field $H_{c}=188 \mathrm{Oe}$ for the LCMO film (a) and $M_{\text {Sat }} \approx 3.66 \mu_{\mathrm{B}} / \mathrm{Mn}$ and $H_{c}=67$ Oe for the LSMO film (b). The Au-NP deposition (red) does affect neither the saturation magnetization nor coercive field of our manganite films indicating an intact bulk behavior. 


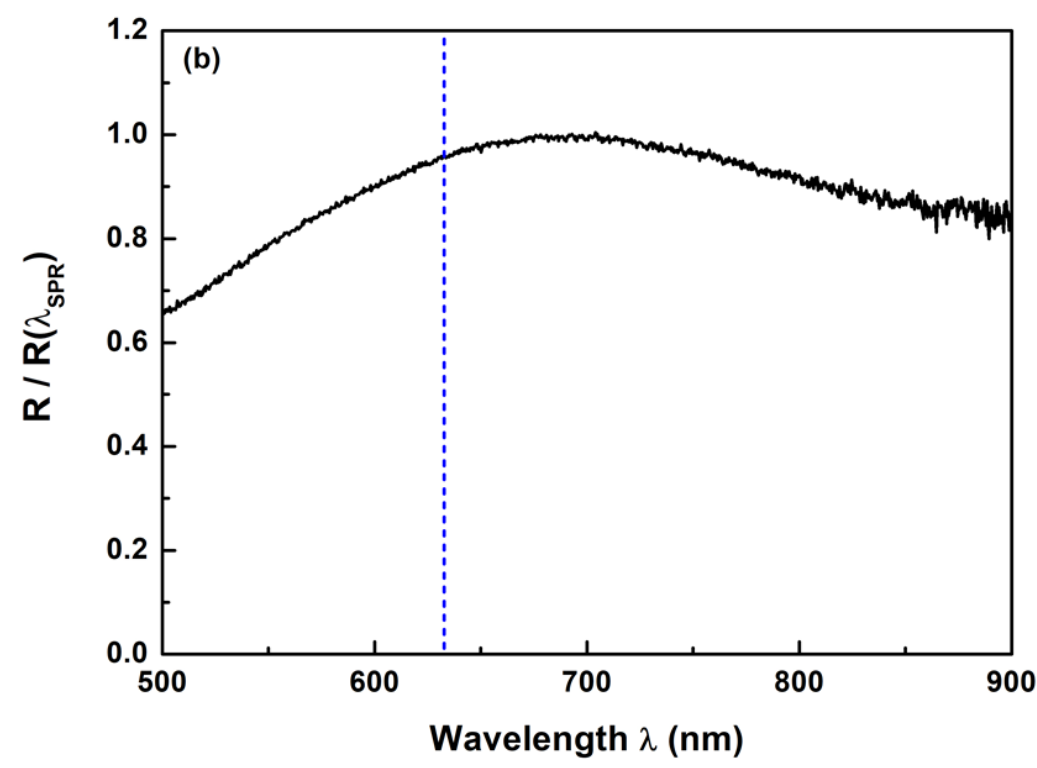

Figure B.5.: The reflectance measurement of the $\mathrm{Au}-\mathrm{NP} / \mathrm{CaMnO}_{3} / \mathrm{LaAlO}_{3}$ (100) film show a SPR frequency, $\lambda_{\mathrm{SPR}}=693 \mathrm{~nm}$. The dashed blue line indicates the excitation wavelength, $\lambda=632.8 \mathrm{~nm}$.

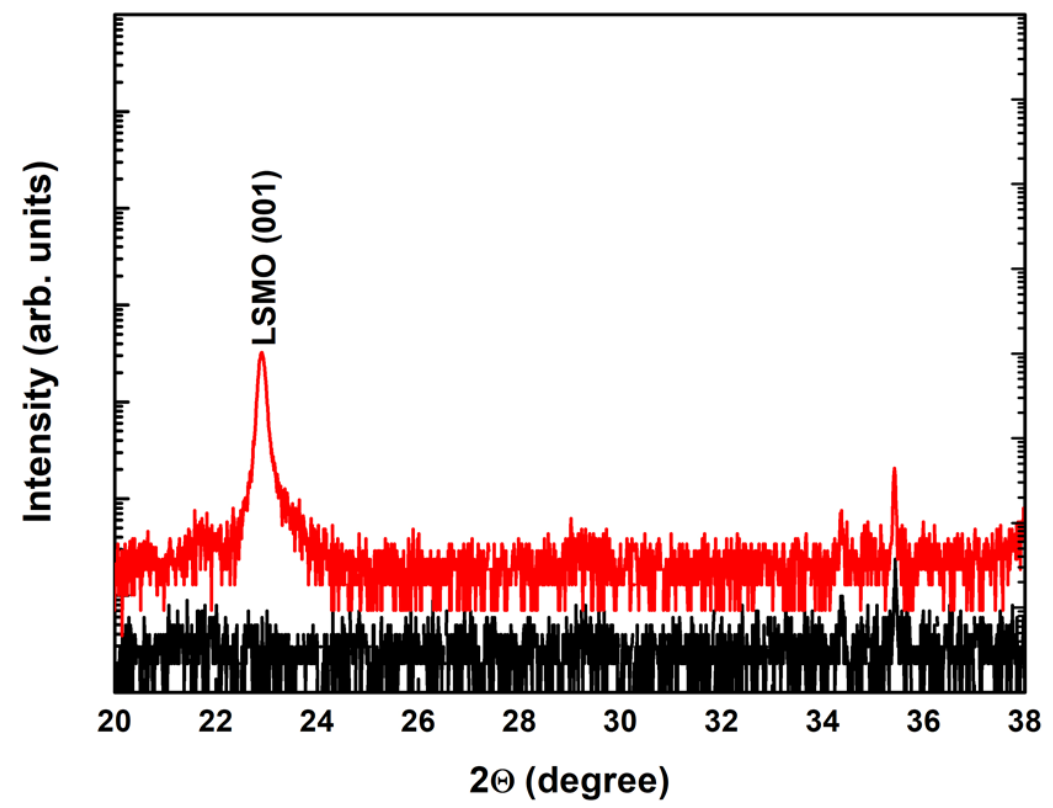

Figure B.6.: XRD pattern of a blank $\mathrm{MgO}(100)$ substrate (black) and a manganite film (red). 


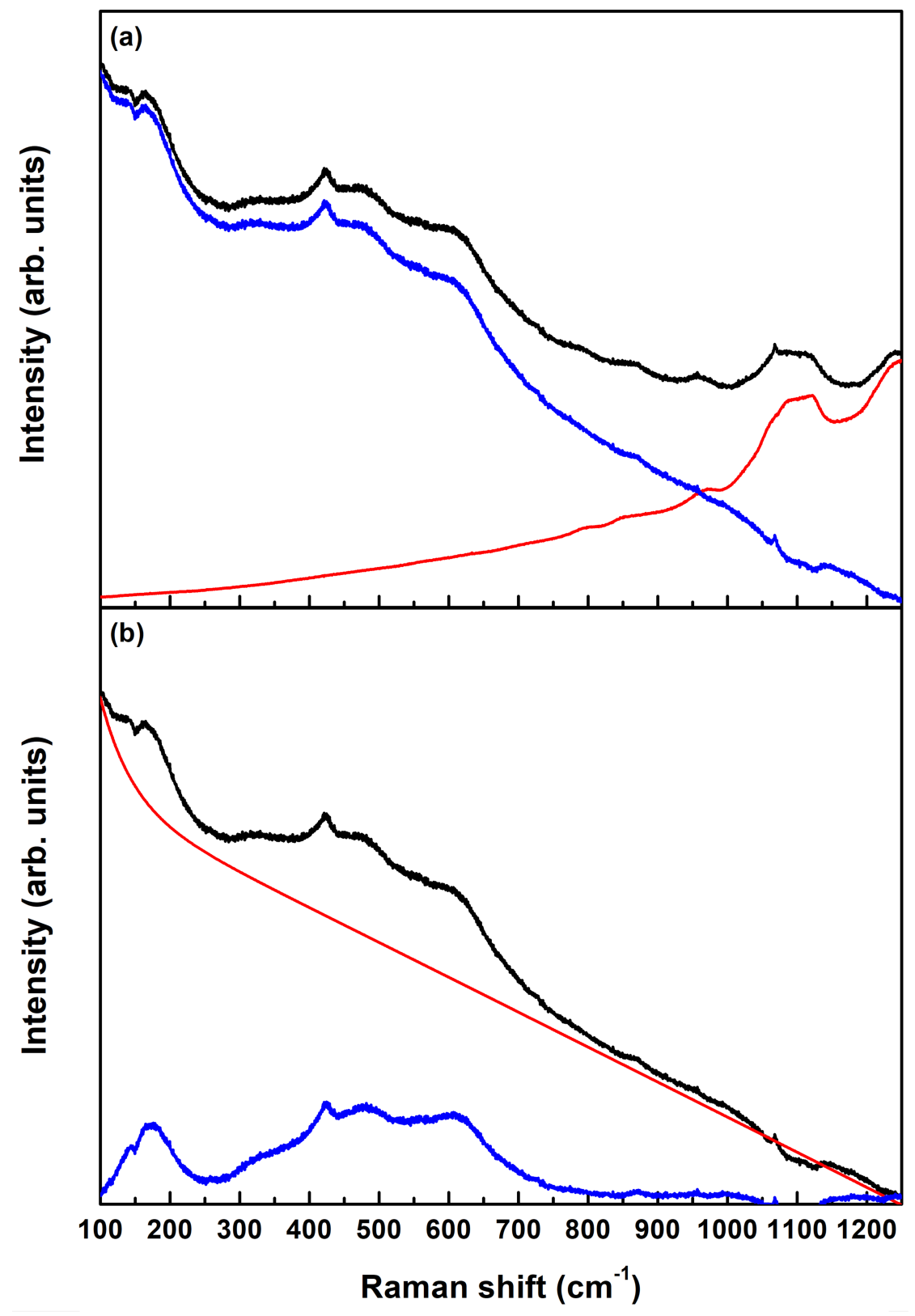

Figure B.7.: Exemplary subtraction of the substrate contribution from the normalized Raman spectra (a) and the subsequent fitting of the remaining background continuum (b). The feature in the region $1000-1200 \mathrm{~cm}^{-1}$ occurs due to an imperfect substrate subtraction, but lies beyond the relevant spectral region $\left(100-900 \mathrm{~cm}^{-1}\right)$. 


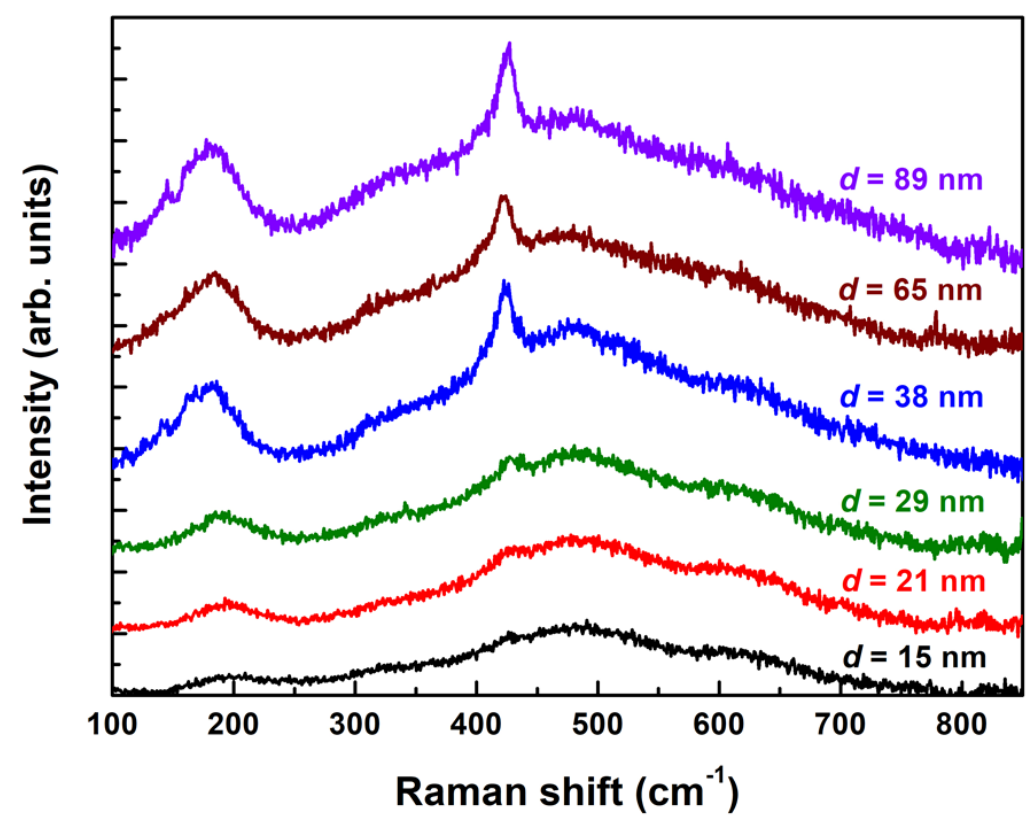

Figure B.8.: Thickness-dependent Raman spectra of $\mathrm{La}_{0.7} \mathrm{Sr}_{0.3} \mathrm{MnO}_{3}$. The intensity of the JT modes decreases within increasing thickness indicating a strong interface contribution to the intensity of those peaks.

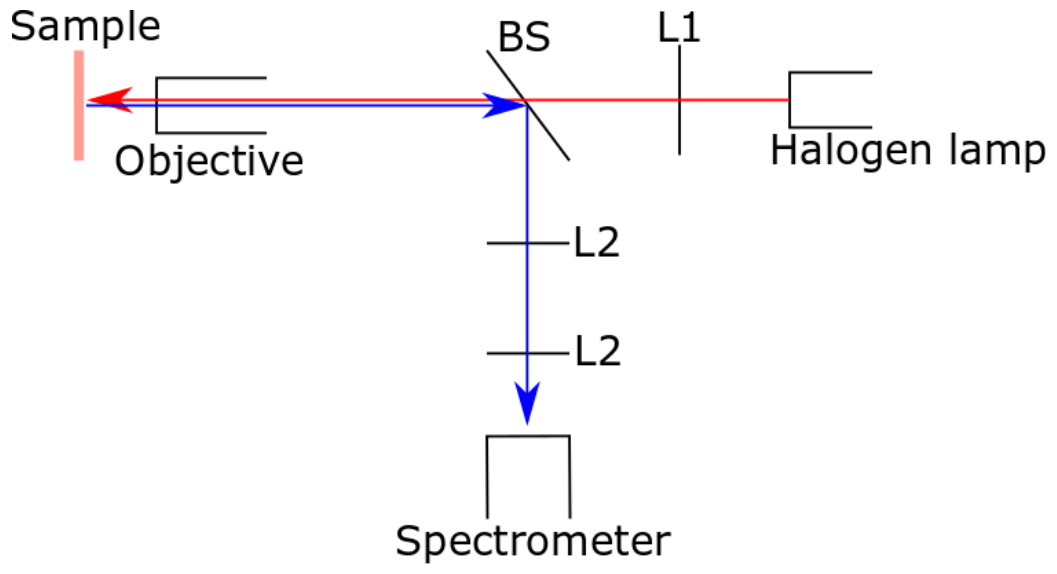

Figure B.9.: Schematic of the optical reflectance setup. 


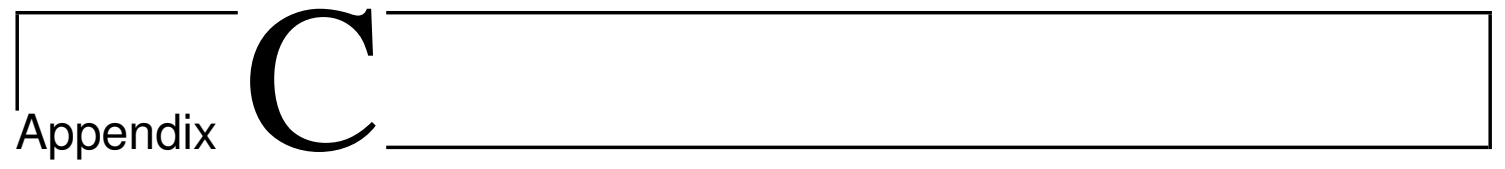

\section{Epitaxial single crystalline growth of $\mathrm{La}_{0.7} \mathrm{Sr}_{0.3} \mathrm{MnO}_{3}$ on $\mathrm{Al}_{2} \mathrm{O}_{3}(0001)$}

Within the scope of this thesis, additional advances in the thin film deposition of $\mathrm{La}_{0.7} \mathrm{Sr}_{0.3} \mathrm{MnO}_{3}$ on $\alpha-\mathrm{Al}_{2} \mathrm{O}_{3}$ were accomplished. However, since the observed properties of these films leave many open questions requiring further characterization, do not fit naturally in the discussion of polaron formation and do not represent methodical advances, contributing to the discussion, the obtained unpublished results are presented in this appendix chapter. While the characterization by means of atomic force microscopy (AFM), x-ray diffraction (XRD), x-ray reflectometry (XRR), electrical and magnetic measurements were performed by the author of this thesis, $\phi$-scan measurements were made in collaboration with Sebastian Esser of the University of Augsburg.

Sapphire substrates $\left(\alpha-\mathrm{Al}_{2} \mathrm{O}_{3}\right)$ possess a hexagonal structure and are of great importance for the integration into electronic devices due to their excellent dielectric, mechanical and optical properties [361]. Moreover, recent developments in silicon-on-sapphire (SOS) technology [414] allow the fabrication of high quality device structures with parameters comparable and, sometimes, even superior to current SI-MOSFET technology [415. The epitaxial growth of functional oxides, such as manganites, can be viewed as an useful advancement for SOS technology allowing a promising integration into microelectronics. However, grown films on c-cut (0001) substrates were found to be polycrystalline or preferentially (111)-textured [416]. Corresponding changes of the magnetic and/or electrical properties due to extrinsic contributions from the interfaces (twining and grain boundaries) could be observed [416]. To the best of our knowledge there is only one publication by V. Bhosle and J. Narayan [417, which claims an epitaxial growth of $\operatorname{LSMO}(110) / \alpha-\mathrm{Al}_{2} \mathrm{O}_{3}(0001)$ films via domain matching epitaxy.

XRD pattern of an LSMO film grown on sapphire (0001) substrate is shown in Fig.C.17. One can see a perfect out-of-plane epitaxy with $\operatorname{LSMO}(111) / \mathrm{Al}_{2} \mathrm{O}_{3}$ orien- 

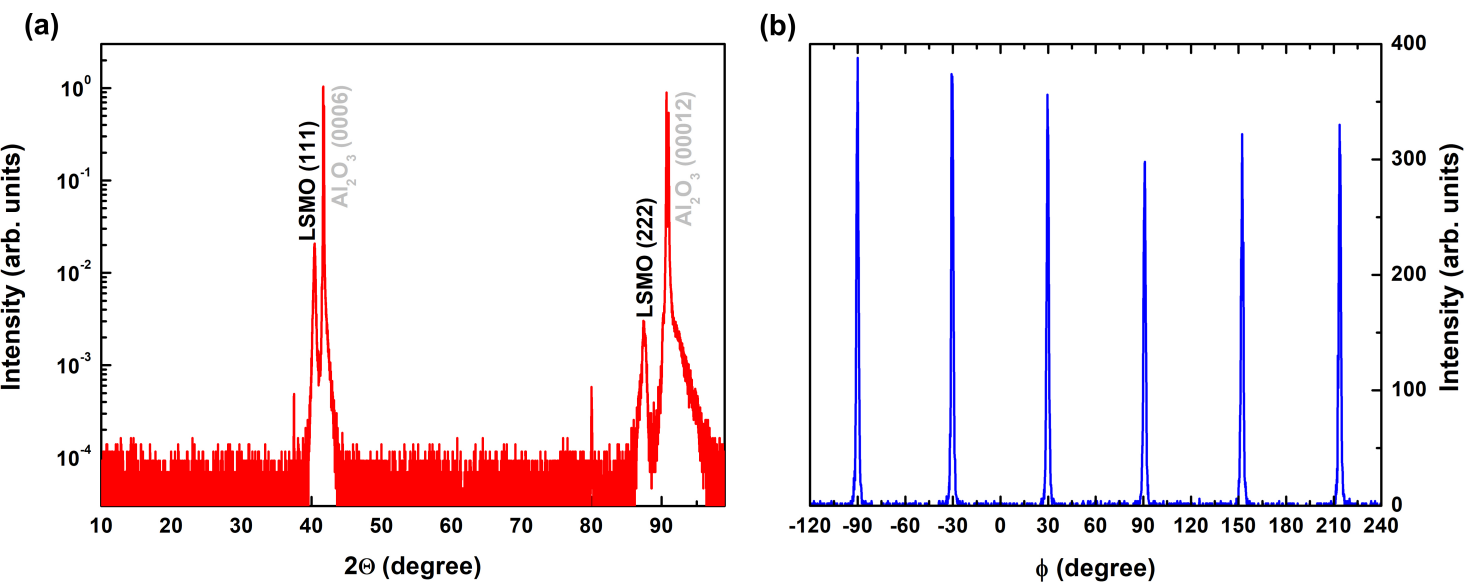

Figure C.1.: XRD pattern (a) and $\phi$-scan (b) reveal an epitaxial growth of LSMO on the $\mathrm{Al}_{2} \mathrm{O}_{3}$ substrates.

tations. No reflexes of a perovskite phase with other orientations as well as reflexes of other phases were observed. Calculated from the measured (111) reflex, pseudocubic lattice constants of the films of $a_{p}=3.862 \AA$ were obtained. In Fig.C.1p the $\phi$-scan of the [112] crystallographic axis demonstrates a 6 -fold rotation symmetry of LSMO grown on the hexagonal structure of $\alpha-\mathrm{Al}_{2} \mathrm{O}_{3}$. Since only the three equivalent [112], [121] and [211] crystallographic axis are crossing the (111) plane, the observed in-plane epitaxy with 6-fold symmetry can be rationalized by the coexistence of two crystallographic pseudo-cubic LSMO(111) domains (twins). These domains are rotated with respect to each other by $180^{\circ}$ and epitaxially grown on $\mathrm{Al}_{2} \mathrm{O}_{3}$.

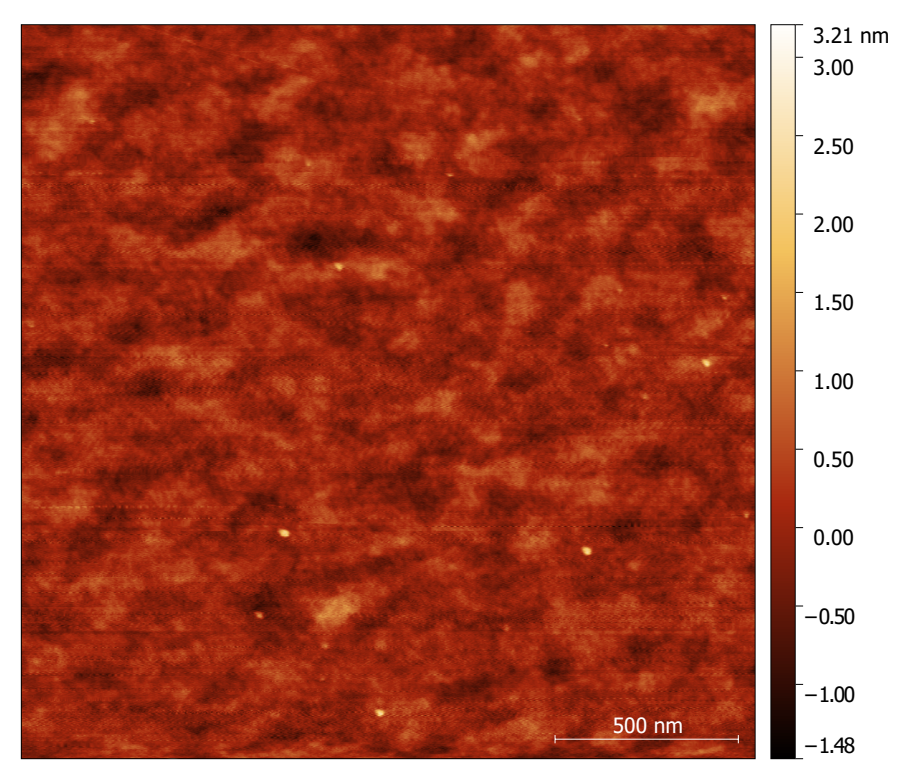

Figure C.2.: AFM images depict a smooth surface morphology with a mean-squareroughness $\mathrm{RMS}=0.2-0.3 \mathrm{~nm}$. 
A typical AFM image of $\mathrm{LSMO}(111) / \mathrm{Al}_{2} \mathrm{O}_{3}(0001)$ films grown by MAD is presented in Fig. C.2. The measured $2 \times 2 \mu \mathrm{m}^{2}$ area shows a smooth surface morphology with a mean-square-roughness RMS $=0.2-0.3 \mathrm{~nm}$. Temperature-dependent magnetization $M(T)$ and electrical resistivity $\rho(T)$ measurements of a representative LSMO film are shown in Fig.C.3. The film demonstrates a para-ferromagnetic transition at $T_{\mathrm{C}}=361 \mathrm{~K}$, determined from the minimum of the function TCM $=1 / M(\mathrm{~d} M / \mathrm{d} T)$ and shown in the inset of Fig.C.3a. The resistivity curve shows a metal-insulator transition around $T_{\mathrm{MI}} \approx 361 \mathrm{~K}$, thus, revealing a coupled metal-insulator paraferromagnetic transition of the studied film. However, a relatively high residual resistance of $\rho \approx 10^{-3} \Omega \mathrm{cm}$ is obtained, which is at least one order of magnitude lower than that obtained in epitaxial films grown on STO [418]. Furthermore, the spin only moment of $3.62 \mu_{\mathrm{B}} / \mathrm{Mn}$, deduced from magnetization measurements at $T=5 \mathrm{~K}$, is in good agreement with the expected value for a Sr-doping of $x=0.3$. The coercive field at $T=5 \mathrm{~K}$ is found to be $H_{c}=56$ Oe (see Fig.C.4 and is, therefore, nearly three times larger than the one calculated in films grown on STO $\left(H_{c}=20\right.$ Oe [168]). Both, the high residual resistivity and the large coercive field, indicate a defect-rich microstructure of the grown $\operatorname{LSMO}(111) / \mathrm{Al}_{2} \mathrm{O}_{3}(0001)$ film, which is further supported by the resistivity increase at low temperatures.

(a)

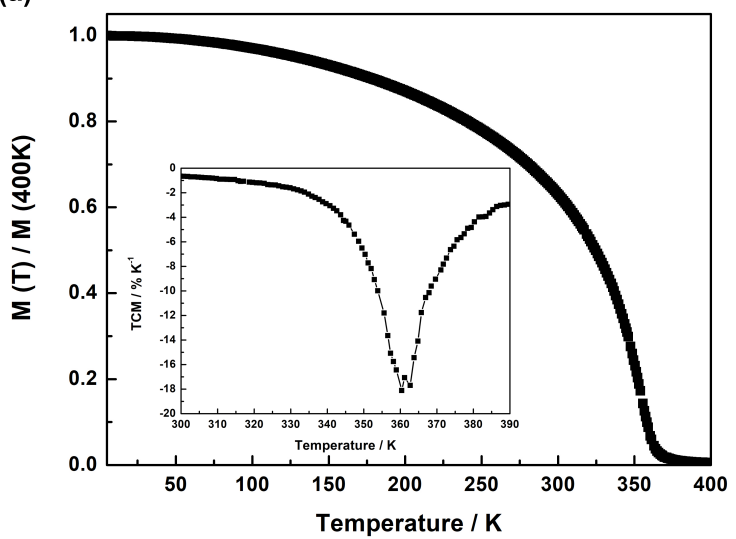

(b)

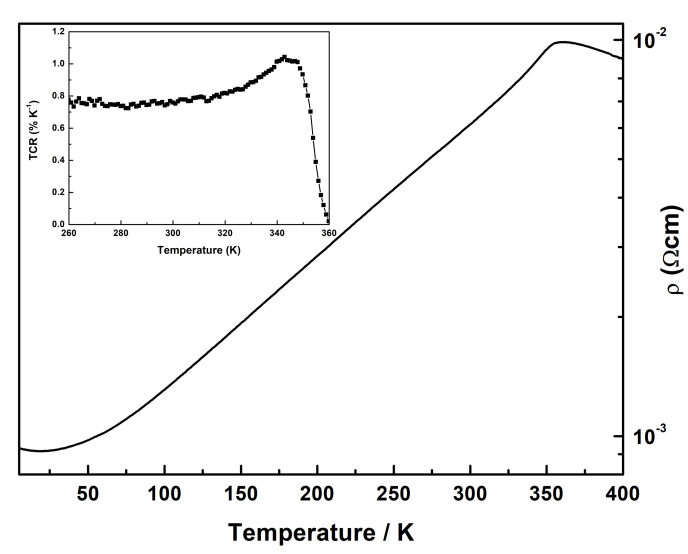

Figure C.3.: The $M(T)$ curve (a) and $\varrho(T)$ curve (b) exhibit a coupled metalinsulator para-ferromagnetic transition with a transition temperature $T_{\mathrm{C}}=T_{\mathrm{MI}}=361 \mathrm{~K}$.

Nonetheless, the results point out that fully epitaxial LSMO(111)-oriented films can be prepared on $c$-cut $\mathrm{Al}_{2} \mathrm{O}_{3}(0001)$ substrates by using MAD. Considering the same hexagonal symmetry of the oxygen atoms within the (111)-plane and (0001)plane of the perovskite and sapphire, respectively, an almost perfect geometrical structural matching could be found as shown in Fig.C.5. The epitaxial misfit can be determined by comparing the lengths of two geometrically similar triangles with 


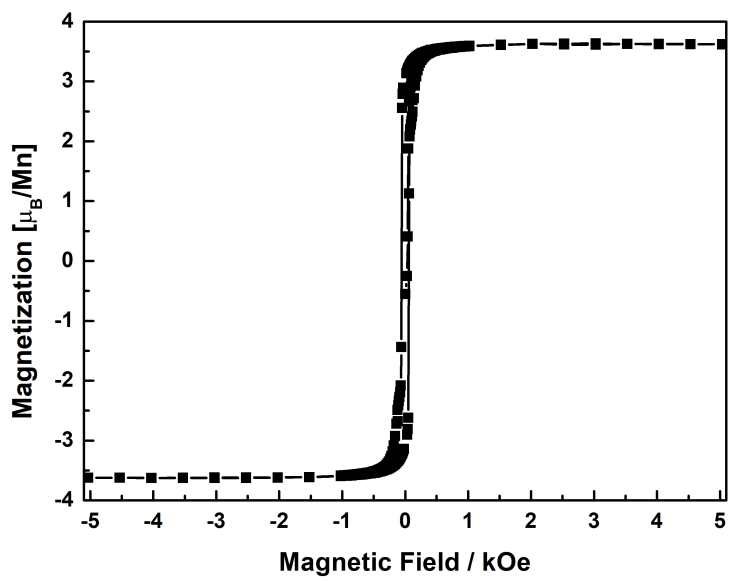

Figure C.4.: $M(H)$ measurements at $T=5 \mathrm{~K}$ show a coercive field of $H_{c}=56$ Oe and a spin only moment of $3.62 \mu_{\mathrm{B}} / \mathrm{Mn}$.

O-atoms at the corners of the perovskite and sapphire, yielding

$$
\epsilon=\frac{\sqrt{3} a_{p} / 2-a_{s}}{a_{s}}
$$

With the determined pseudo-cubic lattice constant $\left(a_{p}=3.862 \AA\right)$ and the lattice constant of the sapphire substrate $\left(a_{s}=4.758 \AA\right)$ [419], a small lattice misfit of $\epsilon=0.58 \%$ and a tensile strain state can be deduced.
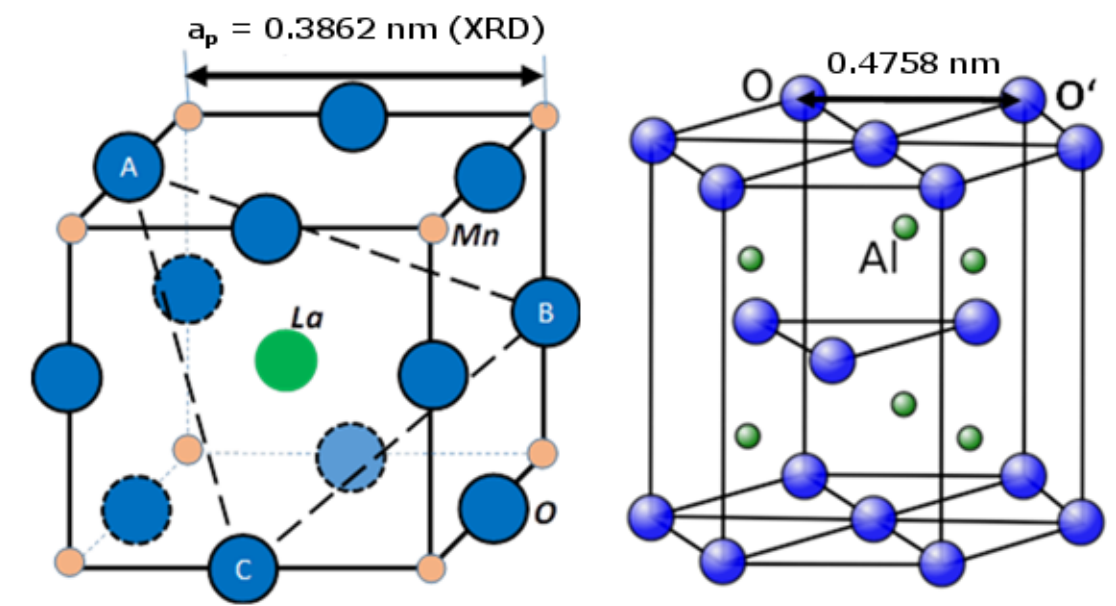

Figure C.5.: Considering the same hexagonal symmetry of the oxygen atoms within the (111)-plane and (0001)-plane of the perovskite and sapphire, respectively, almost perfect geometrical matching could be found.

Although an epitaxial growth of the LSMO film on sapphire was obtained, the properties of the film are far from perfect. Typically, epitaxial grown LSMO films 
exhibit a metal-metal transition around $340 \mathrm{~K}$ and para-ferromagnetic transition at $360 \mathrm{~K}$. While the magnetic properties of the grown LSMO films are in good agreement with those obtained for epitaxial films grown on STO, the electrical resistivity exhibits a metal-insulator transition instead of the expected metal-metal transition. Such behavior cannot be explained by the calculated strain state, since the tensile strain imposed by STO substrates $(\epsilon=0.8 \%$ [168]) is higher than the lattice misfit present in the LSMO $/ \mathrm{Al}_{2} \mathrm{O}_{3}$ film. Although the XRD pattern and phi-scan indicate an epitaxial growth on the $\mathrm{Al}_{2} \mathrm{O}_{3}$ substrate, the high coercive field and high residual resistivity indicate a defect-rich microstructure (grain boundaries). In a next step, detailed transmission electron microscopy measurements should be performed to analyze the film/substrate interface and crystalline quality of the film. Possible structural interface reconstructions, further twinning or grain boundaries within the film may explain the observed metal-insulator transition. 


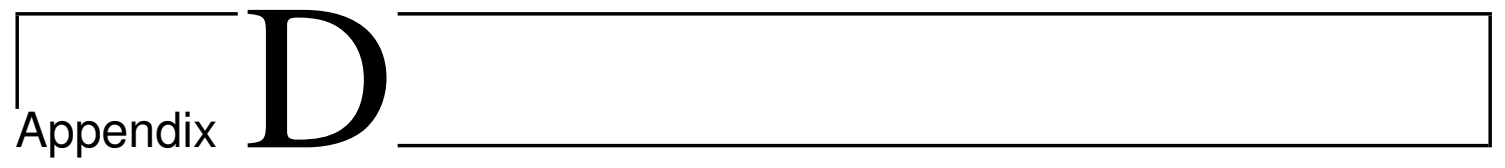

\section{Author Contributions}

The present cumulative thesis consists of the following three research articles of which the author of this thesis is the first author:

1. S. Merten, O. Shapoval, B. Damaschke, K. Samwer, V. Moshnyaga: Magnetic Field Induced Suppression of Jahn-Teller Phonon Bands in $\left(\mathrm{La}_{0.6} \mathrm{Pr}_{0.4}\right)_{0.7} \mathrm{Ca}_{0.3} \mathrm{MnO}_{3}$ : the Mechanism of Colossal Magnetoresistance shown by Raman spectroscopy.

Scientific Reports 9:2387 (2019)

2. S. Merten, V. Roddatis, V. Moshnyaga: Metalorganic-aerosol-deposited Au nanoparticles for the characterization of ultrathin films by Surface-Enhanced Raman Spectroscopy.

Applied Physics Letters 115:151902 (2019)

3. S. Merten, V. Bruchmann-Bamberg, B. Damaschke, K. Samwer, V. Moshnyaga: Jahn-Teller reconstructed surface of the doped manganites shown by means of surface-enhanced Raman spectroscopy.

Physical Review Materials 3:060401(R) (2019)

All three articles are published in a peer-reviewed, international journal and, therefore, fulfill the criteria for a cumulative PhD thesis according to the ProPhys program within the Georg-August University School of Science (GAUSS) program. Furthermore, a fourth publication, of which the author of this thesis is a co-author, is placed before the above listed publications:

- V. Moshnyaga, A. Belenchuk, S. Hühn, C. Kalkert, M. Jungbauer, O. I. Lebedev, S. Merten, K.-Y. Choi, P. Lemmens, B. Damaschke and K. Samwer: Intrinsic antiferromagnetic coupling underlies colossal magnetoresistance effect: Role of correlated polarons.

Physical Review B 89:024420 (2014)

This publication serves as an introduction to the discussed topic and complements Raman scattering experiments on manganites, which is why this publication will 
be referred to publication/manuscript 1. All publications show original research by the authors. The individual contributions for each paper shall be clarified in the following:

The first manuscript was written and revised, mainly, by V. Moshnyaga, combining the experimental results of several doctoral students. A. Belenchuk and S. Hühn prepared the studied manganite samples, whereas S. Hühn in cooperation with C. Kalkert and M. Jungbauer was further responsible for the magnetoresistance and magnetic measurements, presented in this publication. M. Jungbauer developed the underlying model for the estimation of the antiferromagnetic coupling constant and O.I. Lebedev performed transmission electron microscopy measurements of the manganite films. While the author of this thesis performed and interpreted the shown Raman measurements, K.-Y. Choi and P. Lemmens gave support by the interpretation of these measurements. B. Damaschke and K. Samwer contributed to the discussion and interpretation of all the presented experimental results and, furthermore, supervised C. Kalkert during her doctoral thesis.

To enable magnetic-field-dependent Raman spectra presented in the second manuscript, the author conceived, implemented and tested a new optical path to an optical cryostat within an already existing Raman setup under the supervision of $\mathrm{V}$. Moshnyaga. Moreover, he collected all the shown Raman data and contributed to the interpretation and discussion together with B. Damaschke, K. Samwer and V. Moshnyaga. The sample preparation and characterization was done by O. Shapoval. The author wrote the article with the help of V. Moshnayaga.

For the third manuscript, the author of this thesis collected all the presented Raman spectra and fabricated all of the shown $\mathrm{TiO}_{2}$ films as well as the gold nanoparticles. He further performed the sample characterization by x-ray diffraction, scanning electron microscopy and optical measurements, whereas V. Roddatis provided the transmission electron microscope images. The author contributed to the interpretation and discussion of all the measured data. The manuscript was written mainly by the author with the help of V. Moshnyaga and revised by the author.

The author prepared, with the help of V. Bruchmann-Bamberg, the studied manganite films as well as the gold nanoparticles used for the experiments presented in the fourth paper. Moreover, he performed the structural characterization by x-ray reflectometry, x-ray diffraction and scanning electronmicroscopy as well as the electrical, magnetic and optical characterization. The conventional Raman and SER spectra were acquired by the author, who had the main responsibility for the interpretation and discussion of the collected data with the help of B. Damaschke, K. Samwer and V. Moshnyaga. This paper was mainly written by the author with the support of $\mathrm{V}$. Moshnyaga and revised by the author. 


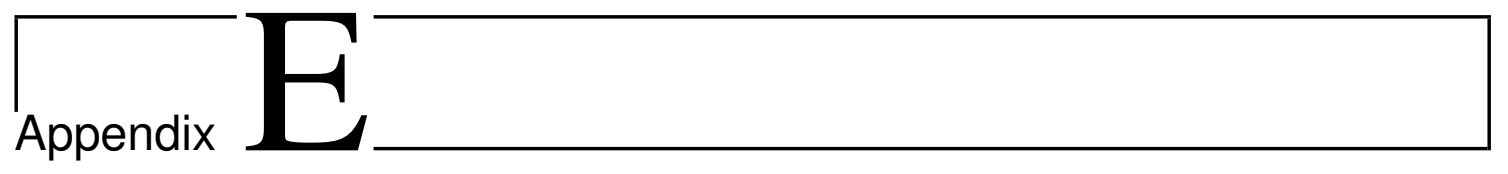

\section{List of Publications}

1. V. Moshnyaga, A. Belenchuk, S. Hühn, C. Kalkert, M. Jungbauer, O. I. Lebedev, S. Merten, K.-Y. Choi, P. Lemmens, B. Damaschke and K. Samwer: Intrinsic antiferromagnetic coupling underlies colossal magnetoresistance effect: Role of correlated polarons.

Physical Review B 89:024420 (2014)

DOI:10.1103/PhysRevB.89.024420

2. C. Meyer, S. Hühn, M. Jungbauer, S. Merten, B. Damaschke, K. Samwer and V. Moshnyaga: Tip-enhanced Raman spectroscopy (TERS) on double perovskite LCMO thin films - Field enhancement and depolarization effects. Journal of Raman Spectroscopy (2016)

DOI:10.1002/jrs.4986

3. S. Esser, C.F. Chang, C.-Y. Kuo, S. Merten, V. Roddatis, T.D. Hale, A. Jesche, V. Moshnyaga, H.-J. Lin, A. Tanaka, C.T. Chen, L.H. Tjeng and P. Gegenwart: Strain-induced changes of the electronic properties of B-site ordered double-perovskite $\mathrm{Sr}_{2} \mathrm{CoIrO}_{6}$ thin films.

Physical Review B 97:205121 (2018)

DOI:10.1103/PhysRevB.97.205121

4. S. Merten, O. Shapoval, B. Damaschke, K. Samwer and V. Moshnyaga: Magnetic-Field-Induced Suppression of Jahn-Teller Phonon Bands in

$\left(\mathrm{La}_{0.6} \mathrm{Pr}_{0.4}\right)_{0.7} \mathrm{Ca}_{0.3} \mathrm{MnO}_{3}$ : the Mechanism of Colossal Magnetoresistance shown by Raman spectroscopy.

Scientific Reports 9:2387 (2019)

DOI:10.1038/s41598-019-39597-1

5. S. Merten, V. Roddatis and V. Moshnyaga: Metalorganic-aerosol-deposited Au nanoparticles for the characterization of ultrathin films by Surface-Enhanced Raman Spectroscopy.

Applied Physics Letters 115:151902 (2019)

DOI:10.1063/1.5121601 
6. S. Merten, V. Bruchmann-Bamberg, B. Damaschke, K. Samwer and V. Moshnyaga: Jahn-Teller reconstructed surface of the doped manganites shown by means of surface-enhanced Raman spectroscopy.

Physical Review Materials 3:060401(R) (2019)

DOI:10.1103/PhysRevMaterials.3.060401 


\section{Acknowledgment}

And as my old english teacher always said, "And now I'm switching to english..". Ok, zugegebenermaßen wechsele ich eigentlich ins deutsch, aber gut. Damit jeder nämlich verstehen kann, wofür ich ihm danken möchte, werde ich die Danksagung auf deutsch formulieren. Denn ohne die folgenenden Leute wäre diese Arbeit nämlich nicht möglich gewesen, weshalb ich ihnen im Folgenden meinen besonderen Dank aussprechen möchte:

- Meinem Doktorvater Prof. Dr. Vasily Moshnyaga für seine Unterstützung, während der Arbeit, die guten (wissenschaftlichen) Diskussionen und die ein oder andere witzige Anektdote.

- Prof. Dr. Claus Ropers für die Übernahme des Koreferats sowie den restlichen Mitgliedern der Prüfungskomission, Prof. Dr. Christian Jooß, Prof. Dr. Michael Seibt, Prof. Dr. Hans Christian Hofsäss, Dr. Martin Wenderoth, dass Sie sich Zeit für meine Disputation genommen haben.

- Den zahlreichen Mitgliedern der Manganatgruppe, die ich in den letzten Jahren kennenlernen und erleben durfte, für die (meist) sehr angenehme Arbeitsatmossphäre. Dabei möchte ich besonders Viktor Pfahl, Camillo Ballani, Marius Keunecke, Cinja Seick, Henrike Probst, Vitaly BruchmannBamberg und Hannes Goedecke hervorheben, die immer wieder mal für ein Bierchen (manchmal ohne Rücksicht auf die Uhrzeit) oder die ein oder andere Ablenkung, während des Arbeitsalltags zu haben waren.

- Meinen Unmengen an Bürokollegen, wie u.a. Danny, Freddy, Eduard(!), Ina, Henrike, Lisa und Daniel, die immer für eine angenehme Arbeitsatmosspähre im Büro sorgten. Ich habe es durch euch nie bereut das Bachelorbüro nicht verlassen zu haben, obwohl ich ihm im wissenschaftlichen Sinne schon längst entwachsen war (im persönliche Sinne kann man drüber streiten, gebe ich zu).

- Meinen fulminanten Kollegen im technischen Bereich, Uta Filippich, Carsten Mahn, Simon Stoldt und Kai Dörner, die einem immer voller Leidenschaft 
geholfen haben, wenn man mal nicht weiter wusste. Ohne euch würde vieles den Bach runtergehen! Hierbei will ich auch den Mitgliedern der Werkstatt, Hartmut Eichenberg und Bastian Beyland, danken, die einem in feinmechanischen Dingen immer gut beraten haben.

- nochmal ausdrücklich Henrike Probst, Vitaly Bruchmann-Bamberg und Karen Stroh für das Korrekturlesen meiner Arbeit (und die ein oder andere schöne Malerei in der Korrektur, nicht wahr Karen?!).

- den Gebrüder Esser, Sebastian und Sven, für die interessanten wissenschaftlichen Diskussionen und den ein oder anderen witzigen Abend bei einem (oder manchmal auch $n$ ) Bierchen.

- sowie allen anderen Mitgliedern des I. Physikalischen Instituts für die freundschaftliche und lockere Arbeitsatmossphähre.

Auch außerhalb der Physik möchte ich bestimmten Personen danken, die mich über all die Jahre hin unterstützt haben:

- Zum einen wären da Alex, Andre, Benni, Jan und Jonas für die langjährige Freundschaft, die vielen schönen und spaßigen Momente, die wir zusammen hatten, haben und hoffentlich noch haben werden. In dem Zusammenhang will ich auch dem Rest der "Familie" danken, Basti, Franzi, Soeke, Melina, Didi, Antje, Laszlo, Sergio, Ole, Steffen und allen anderen, die ich jetzt vergesse. Hoffentlich führt mich mein Weg bald wieder näher nach Bremen, damit die räumliche Distanz zwischen uns wieder etwas abnimmt.

- Nils und Mark (du bis jetzt Göttinger) für die vielen witzigen Stunden, die ein oder andere Frust-Sauferei (Danke Nils!) sowie den vielen Kinobesuche (und das dazugehörige Bierchen im CineCafe). Ohne euch ist Kino meist nur halb so cool!

- Kai und Michelle für ... alles?!. Es war mir immer eine Freude bei euch auf dem Sofa rumzuhängen, mit euch zu kochen, zu zocken, zu trinken, einen Sonnenbrand zu bekommen oder ins Kino zu gehen. Danke für die Decke und den Teddy, ich komme auf jeden Fall wieder vorbei! In diesem Zuge möchte ich auch dir danken, Lisa, für ... ebenso alles! Ohne dich schmeckt ein Eis einfach nur halb so gut und ich hoffe, es werde noch viele weitere dazu kommen!

- sowie allen anderen meiner Göttinger-Freunde wie Simon, Alex, Boy, Jorina, Theresa, Thomas, Tim, Torben und vielen anderen, durch die mir meine bisherige Zeit in Göttingen sehr angenehm in Erinnerung bleiben wird.

- und zu guter Letzt meiner Familie, die mich immer unterstützt hat, wenn es Probleme im Studium, der Schule oder im privaten Leben gab. Papa, über dich könnte ich wahrscheinlich nochmals eine Doktorarbeit schreiben, weshalb ich einfach nur eines sagen möchte: Ich hoffe, ich habe Sie in deinen Augen alle ausreichend fertig gemacht! 
